\title{
VIBRATION-BASED HEALTH MONITORING OF ROTATING SYSTEMS WITH GYROSCOPIC EFFECT
}

\author{
A Thesis \\ presented to \\ the Faculty of California Polytechnic State University, \\ San Luis Obispo
}

\author{
In Partial Fulfillment \\ of the Requirements for the Degree \\ Master of Science in Mechanical Engineering
}

by

Nenad Gavrilovic

December 2014 
(C) 2014

Nenad Gavrilovic

ALL RIGHTS RESERVED 
COMMITTEE MEMBERSHIP

TITLE: $\quad$ Vibration-Based Health Monitoring of Rotating Systems with Gyroscopic Effect

AUTHOR: $\quad$ Nenad Gavrilovic

DATE SUBMITTED: $\quad$ December 2014

COMMITTEE CHAIR: $\quad$ Xi (Julia) Wu, PhD

Associate Professor of Mechanical Engineering

COMMITTEE MEMBER: Frank Owen, PhD

Professor of Mechanical Engineering

COMMITTEE MEMBER: Frank Poehler, PhD

University of Applied Sciences Karlsruhe 


\begin{abstract}
Vibration-Based Health Monitoring of Rotating Systems with Gyroscopic Effect

Nenad Gavrilovic
\end{abstract}

This thesis focuses on the simulation of the gyroscopic effect using the software MSC Adams. A simple shaft-disk system was created and parameter of the system were changed in order to study the influence of the gyroscopic effect. It was shown that an increasing bearing stiffness reduces the precession motion. Furthermore, it was shown that the gyroscopic effect vanishes if the disk of system is placed symmetrically on the shaft, which reduces the system to a JeffcottRotor. The second objective of this study was to analyze different defects in a simple fixed axis gear set. In particular, a cracked shaft, a cracked pinion and a chipped pinion as well as a healthy gear system were created and tested in Adams. The contact force between the two gears was monitored and the 2D and 3D frequency spectrum, as well as the Wavelet Transform, were plotted in order to compare the individual defects. It was shown that the Wavelet Transform is a powerful tool, capable of identifying a cracked gear with a non-constant speed. The last part of this study included fault detection with statistical methods as well as with the Sideband Energy Ratio (SER). The time domain signal of the individual faults were used to compare the mean, the standard deviation and the root mean square. Furthermore, the noise profile in the frequency spectrum was tracked with statistical methods using the mean and the standard deviation. It was demonstrated that it is possible to identify a cracked gear, as well as a chipped gear, with statistical methods. However, a cracked shaft could not be identified. The results also show that SER was only capable to identify major defects in a gear system such as a chipped tooth.

Keywords: Health monitoring, Condition monitoring, Fixed axis gear, Fault detection, Fast Fourier Transform, FFT, Short-Time Fourier Transform, Short-Term Fourier Transform, STFT, 3D FFT, SER, Side Band Energy Ratio, Statistical methods, Wavelet Transform 


\section{TABLE OF CONTENT}

LIST OF TABLES ............................................................................... vii

LIST OF FIGURES .............................................................................. vii

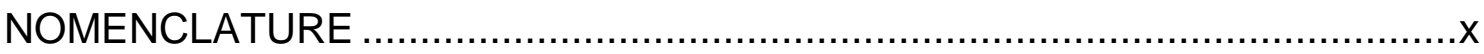

1 The Gyroscopic Effect in Shaft-Disk Systems ........................................... 1

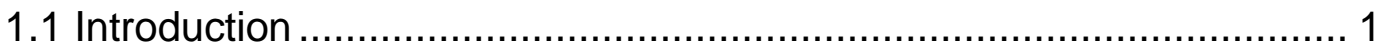

1.2 The Effect on Critical Speeds ...................................................... 2

1.2.1 Equation of Motion ............................................................. 2

1.2.2 The Stiffness Matrix ................................................................ 4

1.3 The Campbell Diagram for Displaying the Critical Speeds ................. 5

2 Introduction to Wavelet Analysis ........................................................... 14

2.1 Fourier Transform for Periodic Signals ........................................... 14

2.2 The Short Time Fourier Transform (STFT) …................................ 19

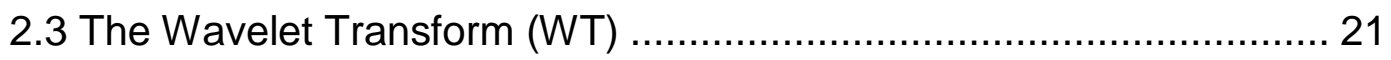

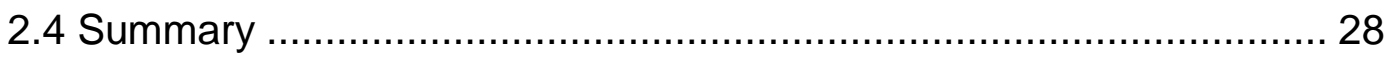

3 Simulation of the Gyroscopic Effect in Adams......................................... 29

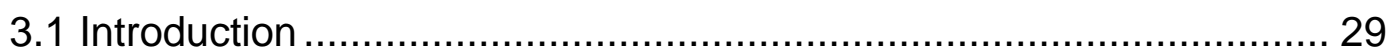

3.2 Variation of the Bearing Stiffness.................................................. 30

3.2.1 Model Setup ................................................................. 30

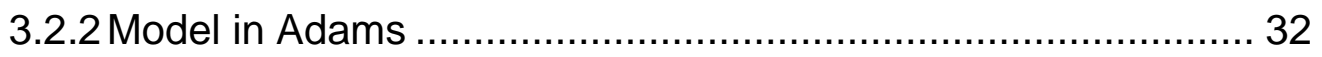

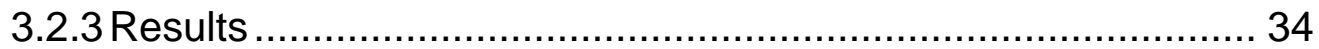

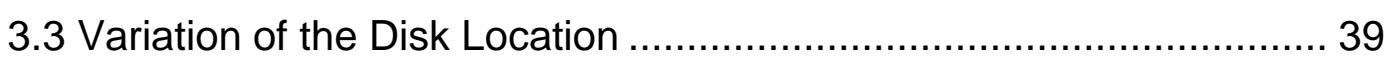

3.3.1 Model Setup ................................................................... 39

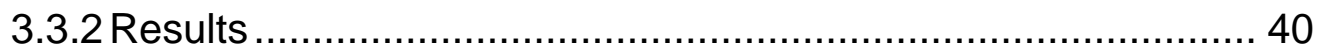

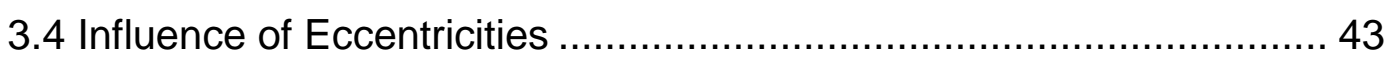

3.5 Analysis of an Anisotropic Bearing System.................................. 45

4 Health Monitoring with a Simple Pair of Gears ......................................... 47

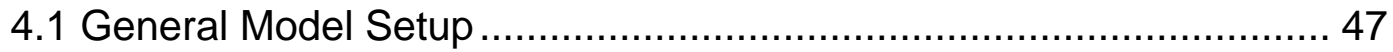

4.2 Modeling the Speed and Torque Profile......................................... 49

4.3 Faults and Defects in the Gear System ....................................... 51

4.4 Simulation Procedure and Analysis Scope .................................... 53

4.5 Analysis of the Flexibility of the Shaft and the Bearings..................... 54

4.6 Health Monitoring with FFT and 3D FFT ..................................... 56

4.6.1 FFT and Time Domain Analysis ............................................... 56

4.6.1.1 Chipped Pinion....................................................... 56

4.6.1.2 Cracked Pinion.......................................................... 58

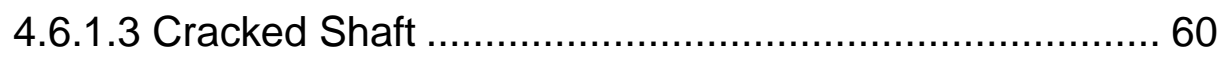

4.6.23D FFT Analysis with a Constant Speed.................................. 62 


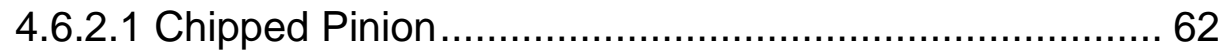

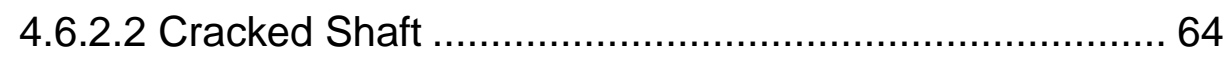

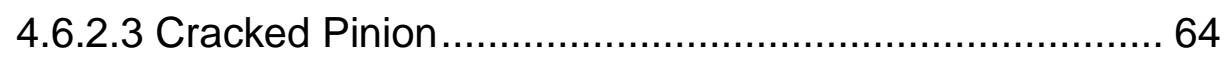

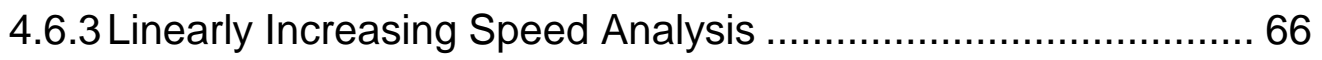

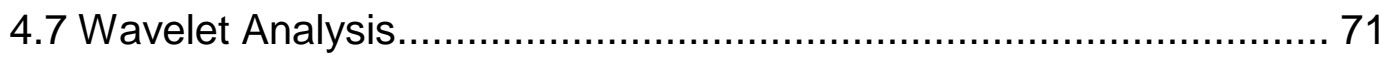

4.7.1 Wavelet Analysis of a Healthy Gear...................................... 71

4.7.2 Wavelet Analysis of a Chipped Pinion...................................... 74

4.7.3Wavelet Analysis of a Cracked Shaft ....................................... 75

4.7.4 Wavelet Analysis of a Cracked Gear ..................................... 76

4.8 Statistical Fault Detection Methods................................................ 77

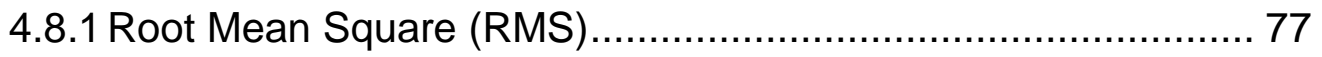

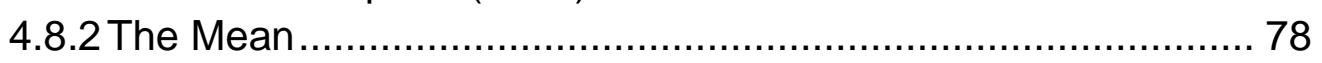

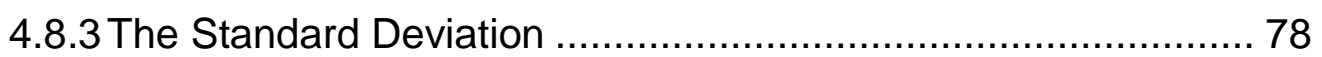

4.8.4 Sideband Energy Ratio (SER) .............................................. 78

4.8.5 Statistical Methods for the FFT Spectrum ………………........ 79

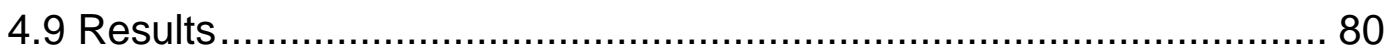

4.9.1 Analysis of the Signal in the Time Domain ............................... 80

4.9.2 Analysis of the Signal in the Frequency Domain ...................... 83

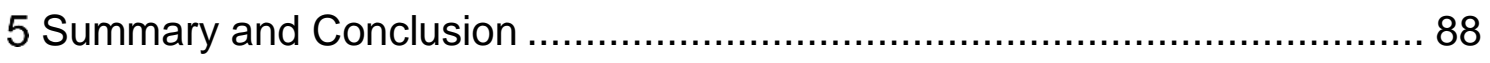

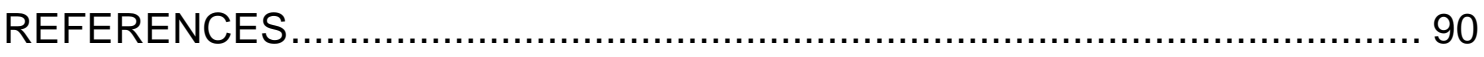

Appendices

A: 3D FFT Results (exponential speed) ............................................. 92

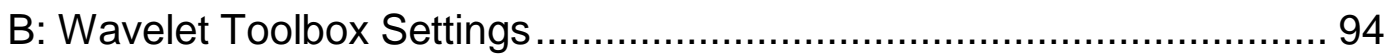

C: Wavelet Linear Increasing Speed - Results ....................................... 95

D: How to Plot Wavelet from ADAMS Results ……….......................... 99

E: Gyroscopic Effect Applied in Rotor Systems ................................. 102

F: How to define a motor torque curve in Adams ............................... 112

G: How to Generate an MNF File ................................................ 115 


\section{LIST OF TABLES}

Table

Page

1.1. Parameter and properties of the shaft-disk model................................... 9

1.2. Comparison of the critical speeds with different flexibilities ............................ 13

3.1. Parameter and properties of the shaft-disk model...................................... 31

3.2. Stiffness values for each simulation ..................................................... 31

3.3. Parameter and properties of the model .................................................... 39

3.4. Disk location ratios for each model....................................................... 40

3.5. Radius and mass of the eccentricities .................................................... 43

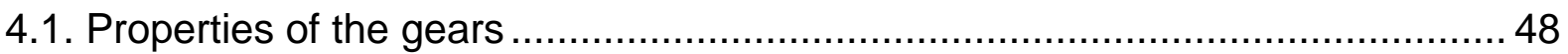

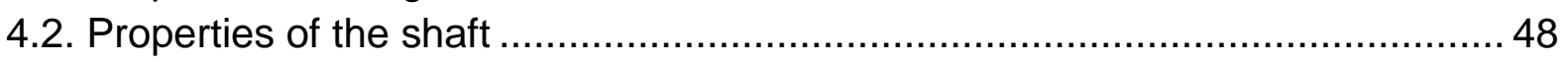

4.3. Numerical values of the GMFs with different flexibilities................................ 55 


\section{LIST OF FIGURES}

Figure

Page

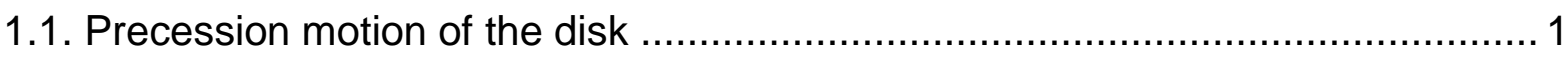

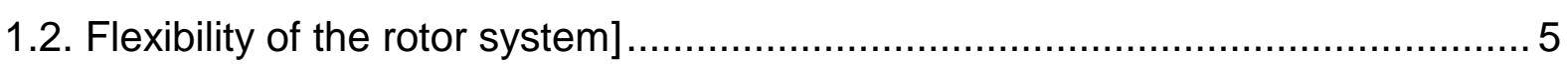

1.3. Campbell Diagram and amplitude-frequency diagram ................................... 6

1.4. Simple drawing of the single disk-shaft model ............................................. 8

1.5. Campbell Diagram with flexible bearings and a rigid shaft ............................. 10

1.6. Campbell Diagram with rigid bearings and a flexible shaft ............................ 11

1.7. Campbell Diagram with rigid bearings and a flexible shaft .............................. 12

2.1. Approximation of a square function with a Fourier series .............................. 15

2.2. Time and frequency domain of a simple sine function................................... 16

2.3. Time and frequency domain of a superimposed function .............................. 17

2.4. Time and frequency domain of a non-periodic function ................................ 18

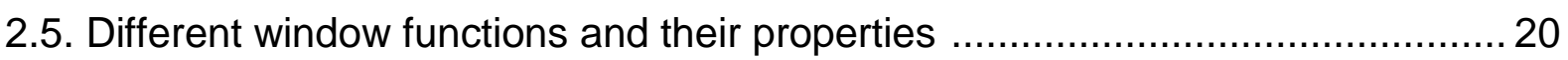

2.6. Wavelet function and the corresponding scaling function................................ 22

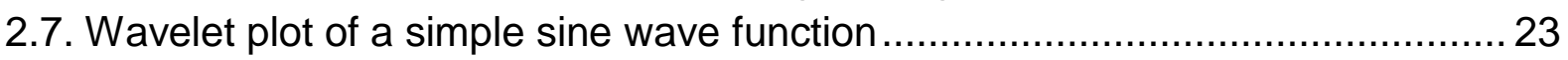

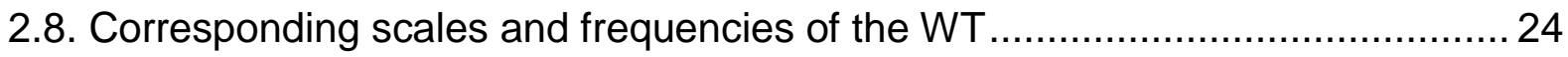

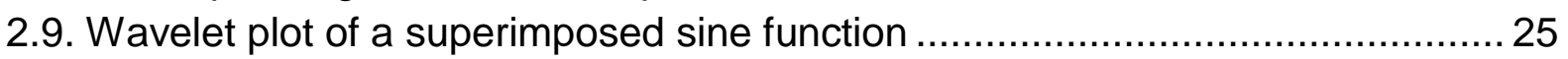

2.10. Wavelet plot of a non-periodic function .................................................. 26

2.11. Wavelet plot of a superimposed sine function with noise ............................. 27

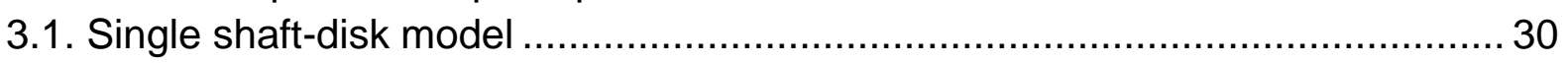

3.2. Adams model of the single shaft-disk system …….................................... 32

3.3. Bushing property manager in MSC Adams .................................................. 33

3.4. Euler Angle (pitching) with different bearing stiffness values ......................... 34

3.5. Euler Angle (pitching) with different bearing stiffness values (zoom) ................ 35

3.6. Euler Angle (yawing) with different bearing stiffness values.............................. 36

3.7. Euler Angle (yawing) with different stiffness values (zoom in) ........................ 37

3.8. Orbit plot of the disk with different stiffness values.......................................... 38

3.9. Euler Angles (pitching) with different disk locations....................................... 40

3.10. Euler angles (yawing) with different disk locations ..................................... 41

3.11. Orbit plots with different disk positions ........................................................ 42

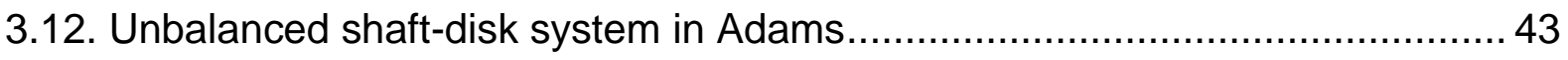

3.13. Orbit plots at $15 \mathrm{~Hz}$ with different eccentricities ........................................ 44

3.14. Frequency response at $15 \mathrm{~Hz}$ with different eccentricities ......................... 45

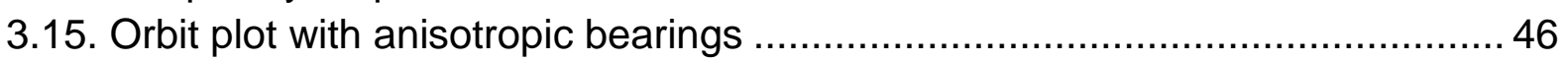

4.1. Simple pair of gears system in Adams ...................................................... 47

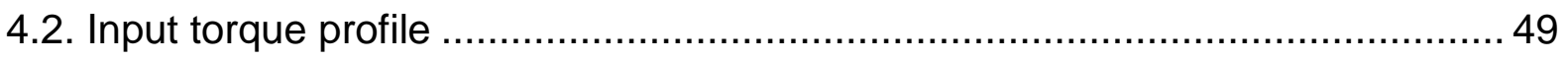

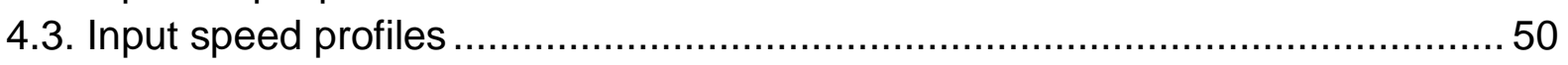

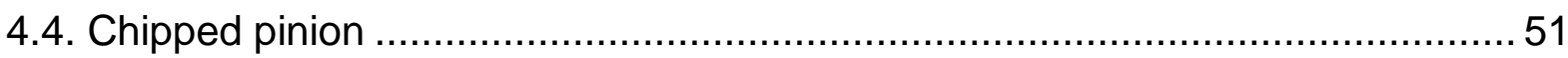

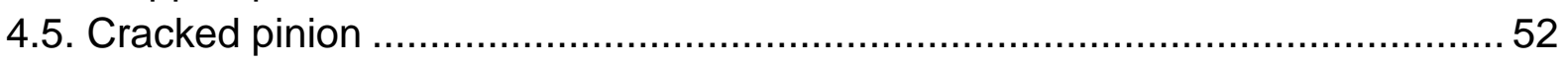

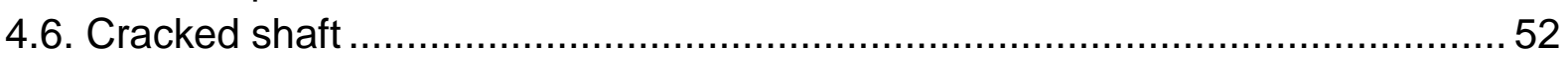




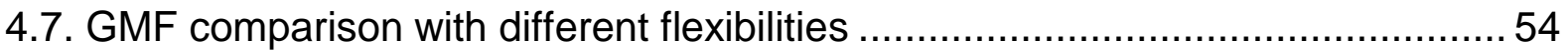

4.8. Contact force in the time domain of the healthy and chipped pinion ................57

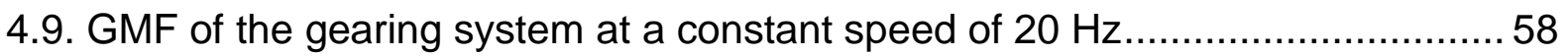

4.10. Contact force in the time domain of a cracked pinion .................................. 59

4.11. Contact force in the frequency domain of a cracked pinion ........................... 59

4.12. Response of the system with a cracked shaft ...........................................6 60

4.13. Comparison of the faults in the frequency spectrum ...................................61

4.14. 3D FFT of a healthy pinion with a constant speed at $20 \mathrm{~Hz} \ldots \ldots \ldots \ldots \ldots \ldots \ldots \ldots . . . . . . . . . . . .62$

4.15. 3D FFT of a chipped pinion with a constant speed at $20 \mathrm{~Hz} \ldots \ldots \ldots \ldots \ldots \ldots \ldots \ldots . . . . . . . . . .63$

4.16. 3D FFT of a cracked shaft with a constant speed at $20 \mathrm{~Hz} \ldots \ldots \ldots \ldots \ldots \ldots \ldots \ldots . . . . . . . . . . . .64$

4.17. 3D FFT of a cracked pinion with a constant speed at $20 \mathrm{~Hz}$....................... 65

4.18. Time domain of a healthy and a chipped pinion with an increasing speed ......66

4.19. Frequency domain of a healthy and a chipped pinion ................................. 67

4.20. 3D FFT of chipped and healthy gear subjected to an increasing speed ..........68 68

4.21. 3D FFT of cracked shaft and a healthy gear set with an increasing speed .....69 69

4.22. 3D FFT of cracked pinion and a healthy gear set with an increasing speed ... 70

4.23. Relation between the number of samples and the time................................ 72

4.24. Correspondence frequency to scale table ............................................... 72

4.25. Wavelet analysis of a healthy gear system with a constant speed at $20 \mathrm{~Hz} \ldots .73$

4.26. Wavelet plot of a chipped pinion with a constant speed at $20 \mathrm{~Hz} \ldots \ldots \ldots \ldots \ldots \ldots . . . .74$

4.27. Wavelet plot of a cracked shaft with a constant speed of $20 \mathrm{~Hz} \ldots \ldots \ldots \ldots \ldots \ldots . . . .75$

4.28. Wavelet plot of a cracked pinion with a constant speed at $20 \mathrm{~Hz} \ldots \ldots \ldots \ldots \ldots \ldots . . . . . .76$

4.29. SER for the first three gear mesh frequencies .......................................... 79

4.30. Definition of three frequency ranges for fault detection analysis .................... 80

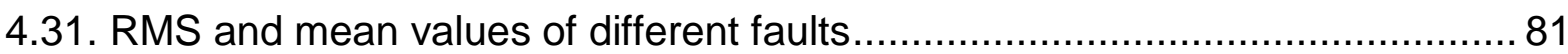

4.32. Standard deviation of different defects ..................................................... 82

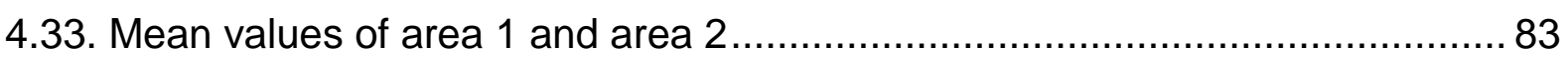

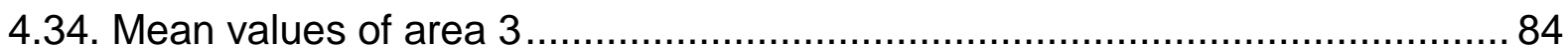

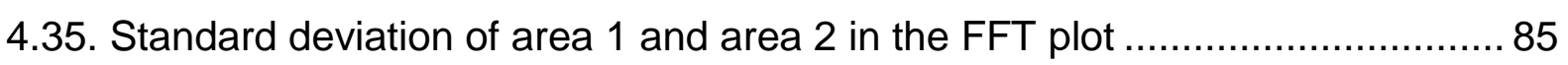

4.36. Standard deviation of area 3 in the FFT plot ........................................... 86

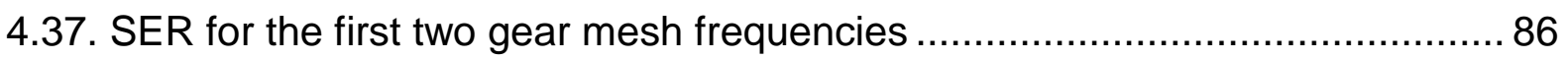

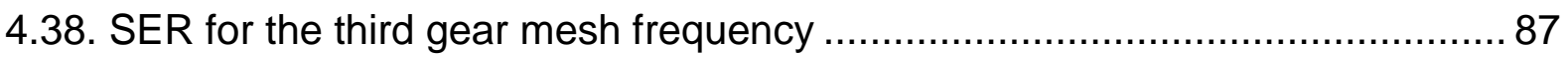




\section{NOMENCLATURE}

English Letters

$a$

$b$

D

$d$

E

F

I

$J_{d}$

$J_{p}$

$j$

$L$

$l$

$t$

$x$

$\ddot{x}$

$y$

$\ddot{y}$ m Distance to disk (left)

m Distance to disk (right)

m Diameter of disk

m Diameter of shaft

$\mathrm{N} / \mathrm{m}^{2} \quad$ Modulus of Elasticity

$\mathrm{N} \quad$ Force

$m^{4} \quad$ Second Moment of Area

$\mathrm{kgm}^{2} \quad$ Moment of inertia of the disk (around $\mathrm{x}$ and $\mathrm{y}$ )

$\mathrm{kgm}^{2} \quad$ Moment of inertia of the disk (around $\mathrm{z}$ )

Complex number

Length of disk

Length of shaft

s Time

m Horizontal displacement of the disk

$\mathrm{m} / \mathrm{s}^{2} \quad$ Horizontal acceleration of the disk

m Vertical displacement of the disk

$\mathrm{m} / \mathrm{s}^{2} \quad$ Vertical displacement of the disk 
Greek Letters

$\begin{array}{lll}\varphi_{x} & \mathrm{rad} & \text { Euler Angle around (horizontal) x-axis } \\ \dot{\varphi}_{x} & \mathrm{rad} / \mathrm{s} & \text { Velocity around (horizontal) x-axis } \\ \ddot{\varphi}_{x} & \mathrm{rad} / \mathrm{s}^{2} & \text { Acceleration around (horizontal) x-axis } \\ \varphi_{y} & \mathrm{rad} & \text { Euler Angle around (vertical) y-axis } \\ \dot{\varphi}_{y} & \mathrm{rad} / \mathrm{s} & \text { Velocity around (vertical) y-axis } \\ \ddot{\varphi}_{y} & \mathrm{rad} / \mathrm{s}^{2} & \text { Acceleration around (vertical) y-axis } \\ \Omega & \mathrm{rad} / \mathrm{s} & \text { Spinning motion } \\ \omega & \mathrm{rad} / \mathrm{s} & \text { Whirling motion }\end{array}$

Abbreviations

DOF

Degree of Freedom

FFT

Fast Fourier Transform

GE

Gyroscopic Effect

MNF

Modal Neutral File

RMS

Root Mean Square

SER

Sideband Energy Ratio

STFT

Short-Time Fourier Transform

WT

Wavelet Transform 


\section{The Gyroscopic Effect in Shaft-Disk Systems}

\subsection{Introduction}

The gyroscopic effect is the most interesting phenomenon in rotor dynamics and occurs whenever an object with an additional body, such as shaft-disk system, is subjected to a rotational motion. Due to inertia effects, the flexibility and the asymmetry of a shaft-disk system, a motion perpendicular to the spinning rotation can occur in the system. This means that the gyroscopic motion is present, when the other axis of the rotor is spinning, which also referred as the procession motion. See Figure 1.1 for details.

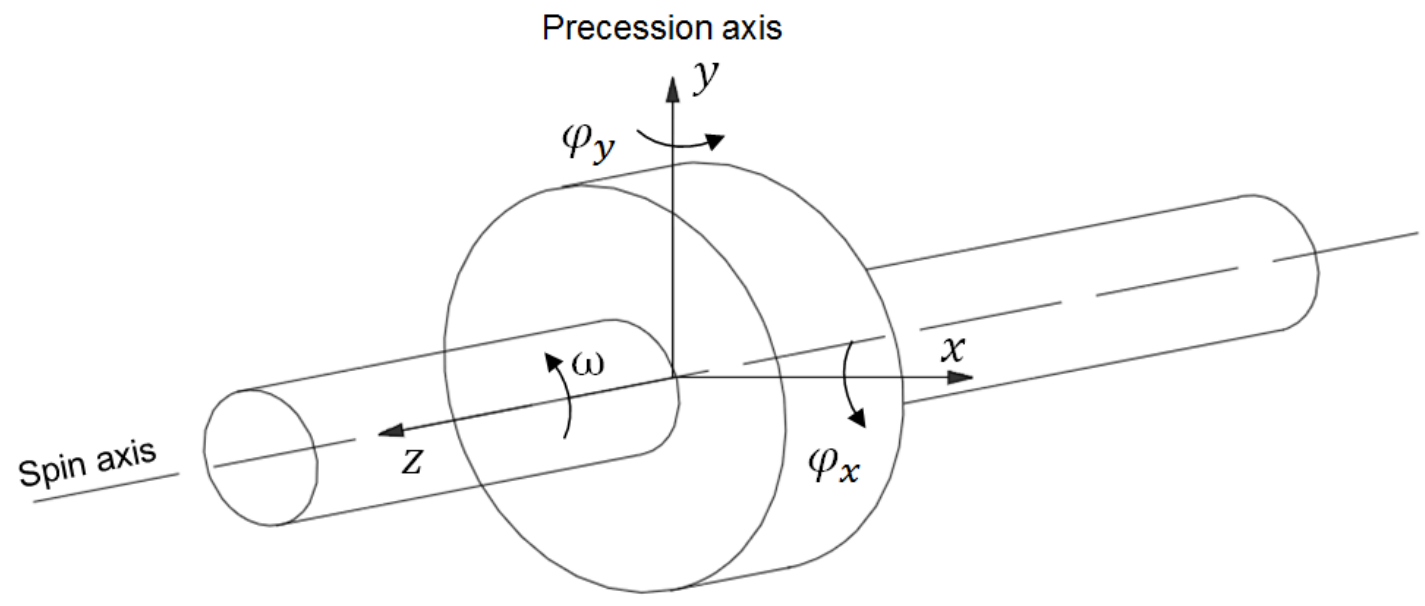

Figure 1.1. Precession motion of the disk

The gyroscopic motion has been in focus of extensive research [1] and can be even considered in fault detection [2]. Therefore, the gyroscopic effect is very important in rotor dynamics and vibrations, since it has a significant influence on the critical speeds of a system, which will be explained in more detail later in this chapter. Thus, it cannot be neglected and must always be taken into account whenever a shaft-disk system is placed on flexible supports. 
Figure 1.1 shows a shaft-disk system with its coordinate system, as well as the spinning axis and the precession axis, caused by the gyroscopic effect. The angles around the $\mathrm{x}$ - axis $\varphi_{x}$ and the $\mathrm{y}$-axis $\varphi_{y}$ are called the Euler Angles and are a result of the gyroscopic effect. If translational displacements ( $x$ and $y$ ) are included, as well as the angular displacements $\left(\varphi_{x}\right.$ and $\left.\varphi_{y}\right)$ around the disk, the shaft disk system can be reduced to a fourth degree of freedom model [3].

The gyroscopic effect can occur in many practical applications. One of the most important example that represents the gyroscopic effect is the gyroscope. Another example were precession effects occur are in aeronautics. The most common example is the helicopter, where the gyroscopic precession is used to control the pitching of a helicopter [4] .

\subsection{The Effect on Critical Speeds}

\subsubsection{Equation of Motion}

The gyroscopic effect can be described by following equation of motions, which describe the resulting forces and the moments acting on the disk [5]. Where $k$ describes the stiffness, $J_{d}$ describes the moment of inertia around the $\mathrm{x}$ - or $\mathrm{y}$ - axis and $J_{p}$ around the z-axis. If free vibration is introduced the moments and forces are zero.

$$
\begin{gathered}
m \ddot{x}+k_{22} x-k_{23} \varphi_{y}=F_{x} \\
m \ddot{y}+k_{22} y-k_{22} \varphi_{x}=F_{y} \\
J_{d} \ddot{\varphi}_{x}+J_{p} \Omega \dot{\varphi}_{y}+k_{23} y+k_{33} \varphi_{x}=M_{x} \\
J_{d} \ddot{\varphi}_{y}-J_{p} \Omega \dot{\varphi}_{x}-k_{23} x+k_{33} \varphi_{y}=M_{y}
\end{gathered}
$$


It is also possible to introduce complex coordinates $j$ for the above equations, which are shown below [3].

$$
\begin{gathered}
z=x+j y \\
\Psi=\varphi_{y}-j \varphi_{x}
\end{gathered}
$$

Substituting these complex numbers in equation (1.1) to (1.4) the equations of motion can be written in a convenient and neat form:

$$
\begin{gathered}
m \ddot{z}+k_{22} z-k_{23} \Psi=0 \\
J_{d} \ddot{\Psi}-j J_{p} \Omega \dot{\Psi}-k_{23} z+k_{33} \Psi=0
\end{gathered}
$$

These complex equations of motion can be solved by introducing the homogenous solution in order to solve these differential equations:

$$
\begin{aligned}
& z(t)=r e^{j \omega t} \\
& \Psi(t)=\alpha e^{j \omega t}
\end{aligned}
$$

By substituting the homogenous solutions, equations (1.7) and (1.8) can be presented in a matrix form:

$$
\left[\begin{array}{cc}
-m \omega^{2}+k_{22} & -k_{23} \\
-k_{23} & -J_{d} \omega^{2}+J_{p} \Omega \omega+k_{33}
\end{array}\right]\left[\begin{array}{c}
r \\
\alpha
\end{array}\right]=\left[\begin{array}{l}
0 \\
0
\end{array}\right]
$$

The characteristic equation of this equation can be obtained if the determinant of the matrix in equation (1.11) is performed. 


$$
\operatorname{det}\left[\begin{array}{cc}
-m \omega^{2}+k_{22} & -k_{23} \\
-k_{23} & -J_{d} \omega^{2}+J_{p} \Omega \omega+k_{33}
\end{array}\right]=0
$$

The characteristic equation is presented in equation (1.13).

$$
\omega^{4}-\frac{J_{p}}{J_{d}} \Omega \omega^{3}-\left(\frac{k_{33}}{J_{d}}+\frac{k_{22}}{m}\right) \omega^{2}+\frac{J_{p} k_{22}}{m J_{d}} \Omega \omega+\frac{k_{22} k_{33}-k_{23}^{2}}{m J_{d}}=0
$$

This equation represents the whirling motion of the system. The whirling motion can occur in the positive direction $\omega$, as well as in the negative direction $-\omega$ (forward and backward whirling). When substituting $\omega$ in (1.13) the natural frequency can be calculated with equation (1.14).

$$
\omega^{4}\left(1-\frac{I_{p}}{I_{d}}\right)-\left(\frac{k_{33}}{I_{d}}+\frac{k_{22}}{m}-\frac{I_{p} k_{22}}{m I_{d}}\right) \omega^{2}+\frac{k_{22} k_{33}-k_{23}^{2}}{m I_{d}}=0
$$

If the negative whirling $-\omega$ is substituted for spinning speed $\Omega$ another equation can be obtained with which the natural speeds for the backward whirling can be calculated.

$$
\omega^{4}\left(1+\frac{I_{p}}{I_{d}}\right)-\left(\frac{k_{33}}{I_{d}}+\frac{k_{22}}{m}+\frac{I_{p} k_{22}}{m I_{d}}\right) \omega^{2}+\frac{k_{22} k_{33}-k_{23}^{2}}{m I_{d}}=0
$$

\subsubsection{The Stiffness Matrix}

The stiffness components of the shaft can be presented in a matrix form. To obtain the individual components, the rotor system has to be assume as a flexible body first, which is shown in Figure 1.2 a. The stiffness components of this matrix can be obtained using the displacement influence coefficients, which are described in [6]. Next, the flexible influence coefficient method of the flexible bearings and rigid shaft model, shown in Figure $1.2 \mathrm{~b}$, has to be used in order to obtain the stiffness components for this 
matrix. Lastly, the two stiffness matrixes of both cases have to be superimposed in order to create the model with flexible bearings and flexible shaft, Figure $1.2 \mathrm{c}$.
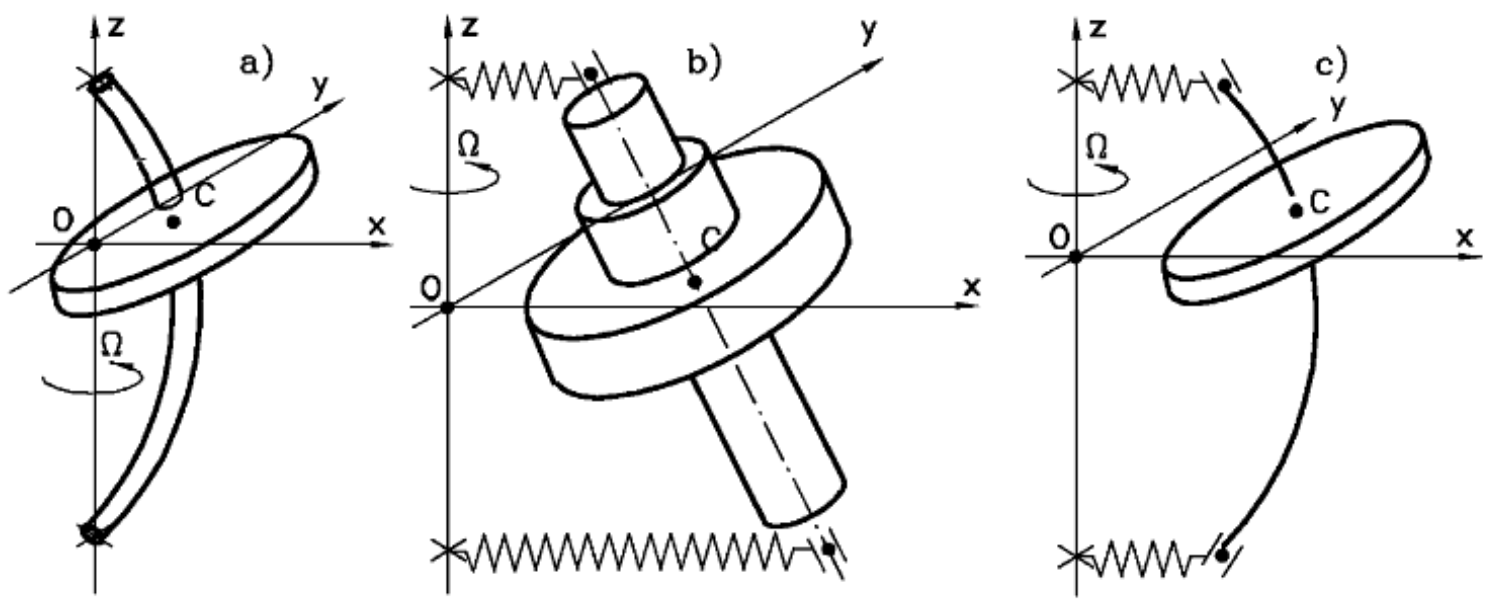

Figure 1.2. Flexibility of the rotor system [3]

\subsection{The Campbell Diagram for Displaying the Critical Speeds}

Vibrational systems can be characterized by critical frequencies, which are important parameter for any rotor system. Reaching critical speeds can result in resonance, which is highly to avoid since it can cause failures to machines. Identifying theses critical speeds is necessary for every engineer in order to reduce danger and harm to the environment and the machine itself. However, in many cases resonance frequencies are not easy to determine. Critical speeds do not always have to be at a constant value. In fact, they can change during the operation in several applications. For instance, resonance frequencies can vary with the spinning speed.

This speed dependence of critical speeds can be caused by the properties of a system. The stiffness of some bearing system, like fluid film bearings, has the ability to vary with the operational speed or to change with temperature [7]. In addition, the stiffness of some systems can change, the higher the operation speed is. Typically, this effect 
occurs in turbines. Turbo-blade resonance frequencies increase with the rotor speed. This phenomenon is called blade stress stiffening, which occurs with increasing rotor speed [8]. On the other hand, the counterpart to stress-stiffness is spin-softening. Spinsoftening enables a reduction of the natural frequencies, when the rotational speed of a system increases [9].

Nevertheless, not only spin-softening and stress-stiffening have a significant influence on the critical speeds, the gyroscopic effect influences the critical speeds as well. The illustration of critical speeds in terms of rotor speeds are called Campbell Diagrams [8]. A Campbell Diagram is presented in the upper part of Figure 1.3.

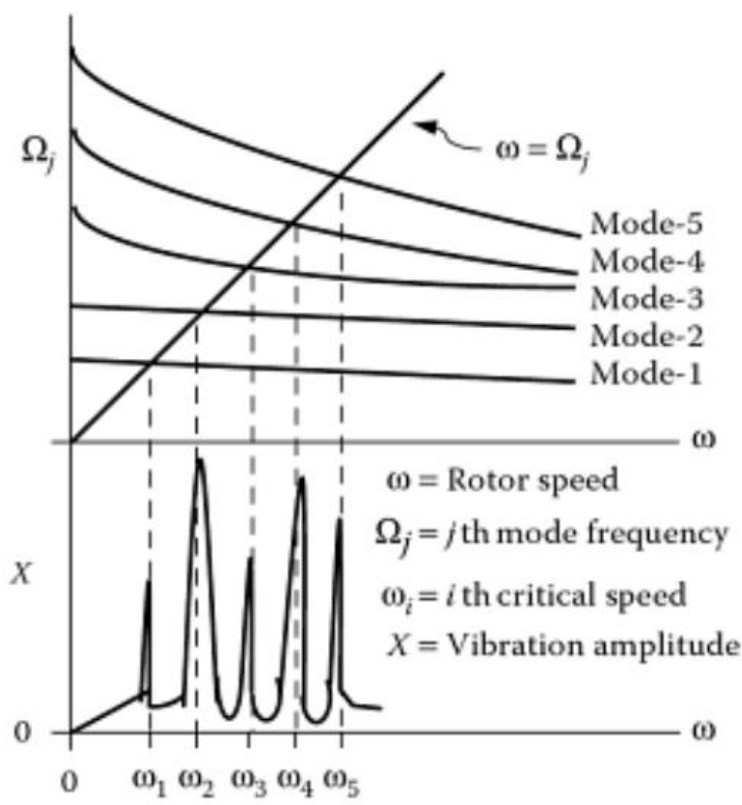

Figure 1.3. Campbell Diagram and amplitude-frequency diagram [8]

The horizontal axis represents the rotor turning speed, while the vertical axis represents the natural frequencies. The lower part of the Campbell Diagram is used as a reference to describe the resonance frequencies in terms of the amplitude and the frequency and to visualize the natural frequencies. The graphs for the different modes, which are the natural frequencies of the system, can be obtained by calculating the imaginary roots of equation 1.13 and plotting them in terms of the operational speed. 
The exciting frequency of the system be can represented as a straight line $\omega=\Omega$ in the Campbell Diagram. Since the relationship between the natural frequency and the exciting speed is in many cases linear, it can be drawn as a line starting from the origin of the plot [10].

The critical speeds can be identified when the line of the exciting frequency hits the lines of the natural frequencies. Every intersections of these two lines can lead to resonance and is strongly to avoid. Once the critical speeds are identified at these intersections, they can be avoided by operating the machine above or below the critical speeds.

Although the Campbell Diagram is a powerful tool for mapping and localizing resonance frequencies it can only provide the location of the critical speeds, but not the corresponding amplitude. Nevertheless, the Campbell Diagram is also suitable for displaying resonance frequencies by considering forward and backward whirling modes.

However, not all identified critical frequencies are equally dangerous. It is likely that some higher modes of the natural frequencies do not appear as significant vibrational peaks in an actual system. It can happen that more critical speeds are found in the Campbell Diagram but do not appear in an actual system. The reason for that might be that some modes are significantly damped and therefore the amplitudes at these resonance modes are not highly developed [8], [10]. Nevertheless, the Campbell Diagram provides engineers the prediction of critical speeds and can be very useful especially in the designing phase of a project. 
In order to demonstrate the meaning and the importance of the Campbell Diagram different example problems will be used. For this, the equation 1.13, which was derived in the previous section, will be used to plot the Campbell Diagram.

First, a system with a rigid body but with a flexible shaft will be used. Next, a disk-shaft system with a rigid shaft but with flexible bearings is going to be used for the analysis scope. Lastly, a system with a flexible shaft and flexible bearings will be used. For all three cases, the critical speeds will be determined using the Campbell Diagram.

In order to display the Campbell Diagram with the natural frequencies the parameter of the single disk model, which is shown in Figure 1.4, needs to be defined. The chosen parameters for the following analysis are presented in Table 1.1.

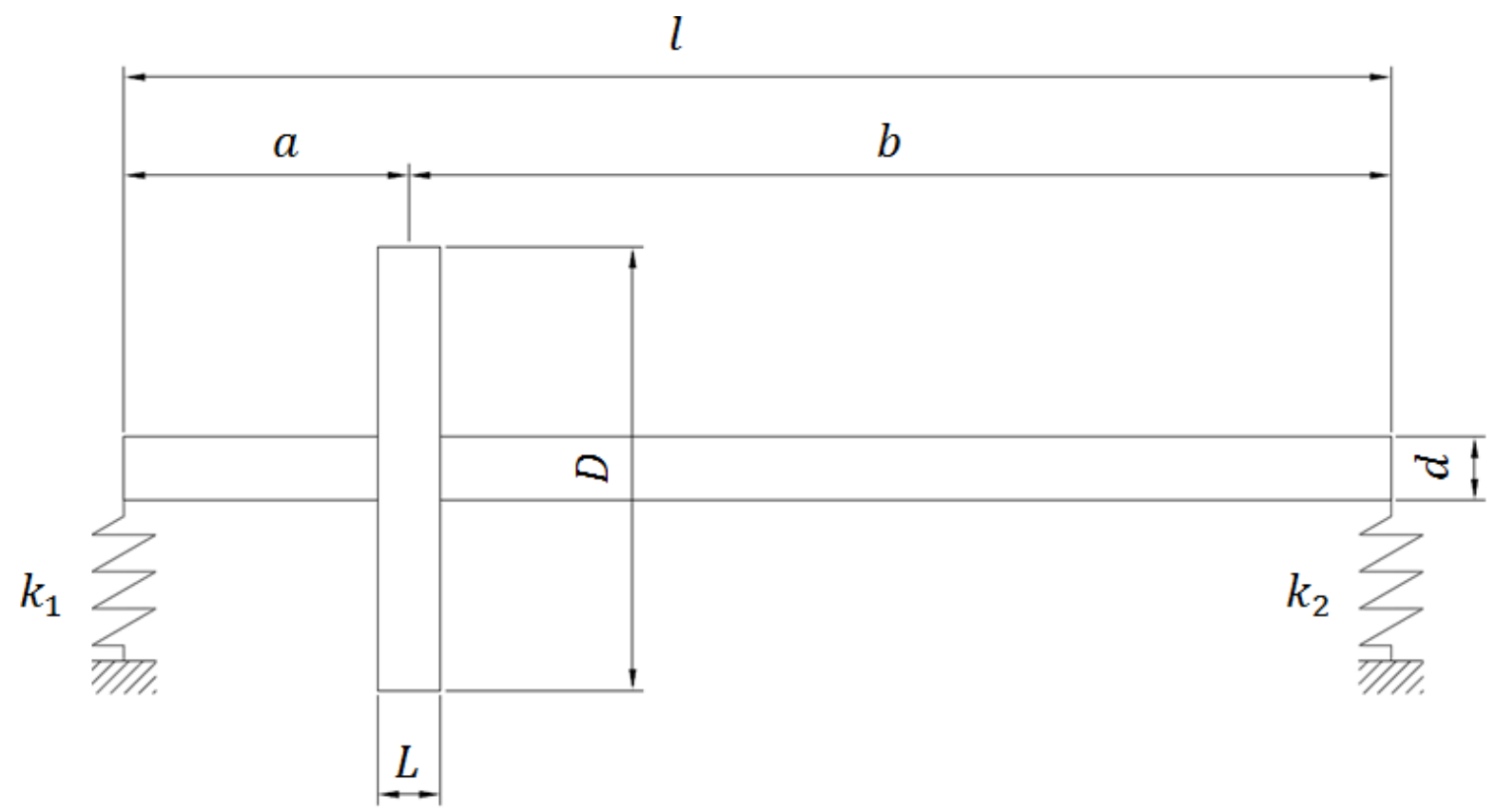

Figure 1.4. Simple drawing of the single disk-shaft model 
Table 1.1. Parameter and properties of the shaft-disk model

\begin{tabular}{cccc} 
Symbol & Parameter name & Value & Unit \\
\hline$a$ & Disk location & 0.125 & $\mathrm{~m}$ \\
$D$ & Diameter of disk & 0.150 & $\mathrm{~m}$ \\
$d$ & Diameter of shaft & 0.01 & $\mathrm{~m}$ \\
$E$ & Modulus of Elasticity & $2.07 \mathrm{E}+11$ & $\mathrm{~N} / \mathrm{m}^{2}$ \\
$L$ & Disk length & 0.02 & $\mathrm{~m}$ \\
$l$ & Shaft length & 0.5 & $\mathrm{~m}$ \\
$k_{1}$ & Stiffness of Bearing 1 & $1.0 \mathrm{E}+07$ & $\mathrm{~N} / \mathrm{m}$ \\
$k_{2}$ & Stiffness of Bearing 2 & $1.0 \mathrm{E}+07$ & $\mathrm{~N} / \mathrm{m}$ \\
$\rho$ & Density & 7,800 & $\mathrm{~kg} / \mathrm{m}^{3}$ \\
$v$ & Poisson ratio & 0.29 & - \\
\hline
\end{tabular}

First, the Campbell Diagram of the rigid-shaft and flexible-bearings of the single diskshaft system will be plotted and analyzed. This means that this model consist of a rigid shaft, which has non-flexible and non-bending characteristics. The stiffness of the bearings are assumed to have a value $1.0 \mathrm{E}+07 \mathrm{~N} / \mathrm{m}$ for each bearing component. This setting makes the bearing to act as a spring and therefore to act as a flexible bearing. 


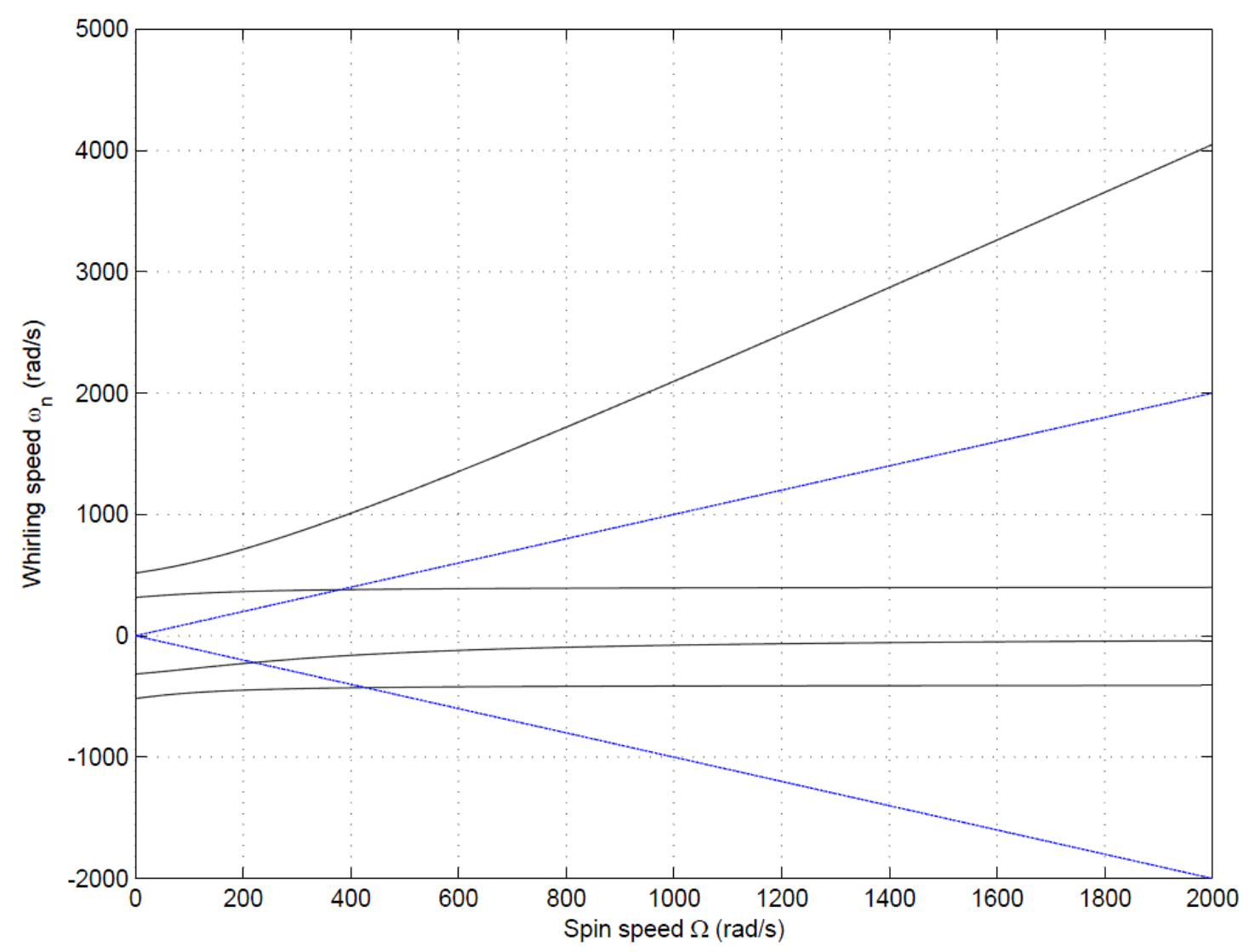

Figure 1.5. Campbell Diagram with flexible bearings and a rigid shaft

The Campbell Diagram for this setup is shown in Figure 1.5. The black lines represent the natural frequencies that vary over time and can be obtained from equating (1.13). The blue lines represent the forcing (exciting) speeds. If the whirling rotation, respectively the whirling speed, of the shaft is in the same direction as the rotation of the shaft (spinning speed) the upper blue line, which lies in the positive direction of the whirling axis, comes into consideration. This determination is valid when forward whirling occurs.

On the other hand, if the whirling of the shaft is in the opposite direction than the movement of the shaft, backward whirling occurs. If this happens, the lower blue line of the forcing speed, which is drawn in the negative axis of the whirling speed, comes into consideration. 
With this method, three different critical speeds can be obtained at every intersection of the forcing speed line and the lines of the natural frequencies $(379.6 \mathrm{rad} / \mathrm{s},-220.5$ $\mathrm{rad} / \mathrm{s}$ and $-427.3 \mathrm{rad} / \mathrm{s})$. Since it is not known if backward or forward whirling occurs in the system, it is strongly to avoid running this setup at these speeds in order to avoid resonance.

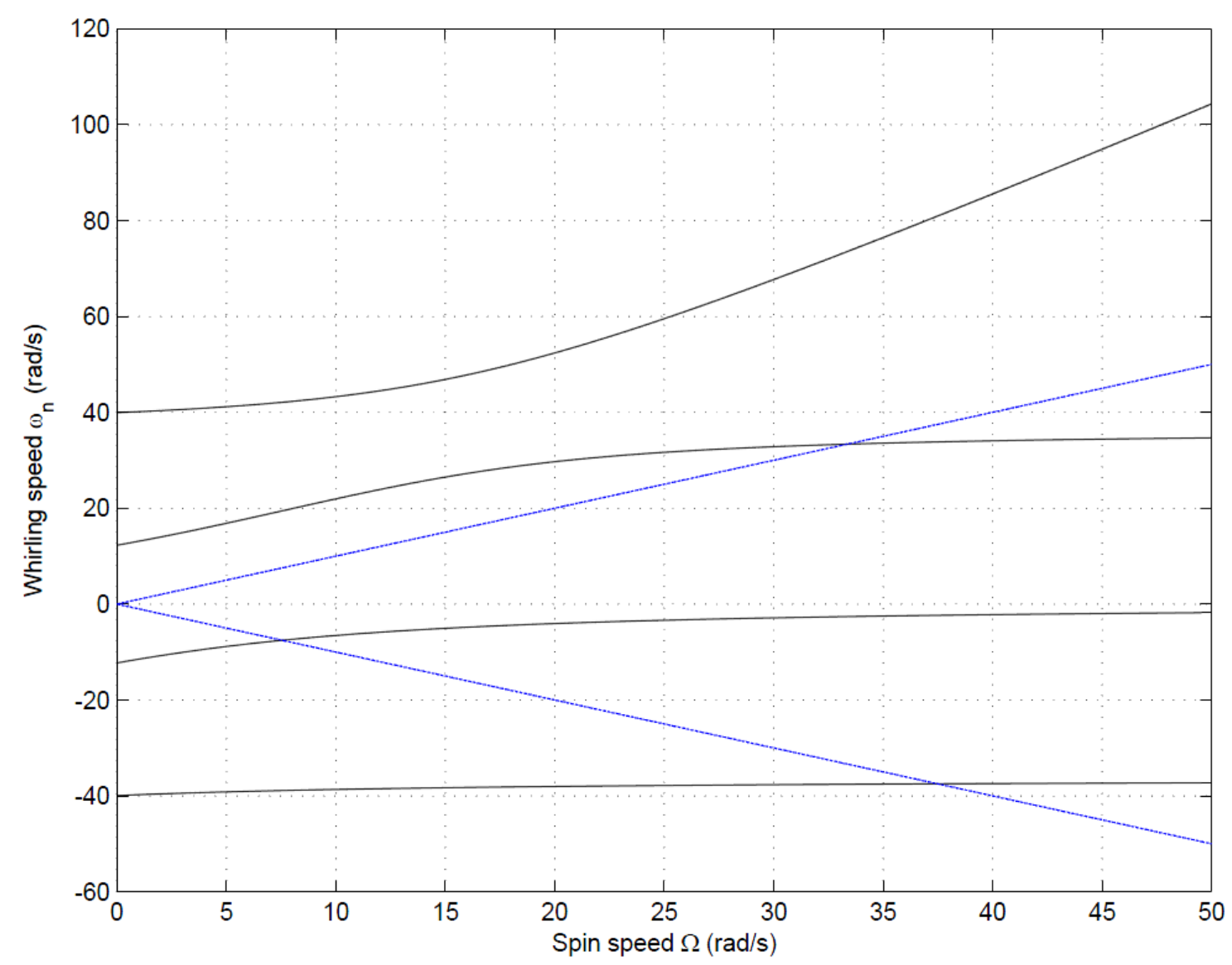

Figure 1.6. Campbell Diagram with rigid bearings and a flexible shaft

Next, the system is assumed to consist a flexible shaft and rigid bearings. The Campbell plot for this configuration is shown in Figure 1.6. Note that for this case compared to the previous example, where the system included a rigid shaft with flexible bearings, the obtained critical speeds reduce significantly. The values of the critical speeds are $33.4 \mathrm{rad} / \mathrm{s},-7.5 \mathrm{rad} / \mathrm{s}$ and $-37.4 \mathrm{rad} / \mathrm{s}$ and again, it is recommend strongly to avoid these specific speed values for this setup. 
In the last configuration, the bearing, as well as the shaft, are assumed to be flexible. For this model, the superposition method must be applied in order to calculate the stiffness of the system. The result is shown in Figure 1.7.

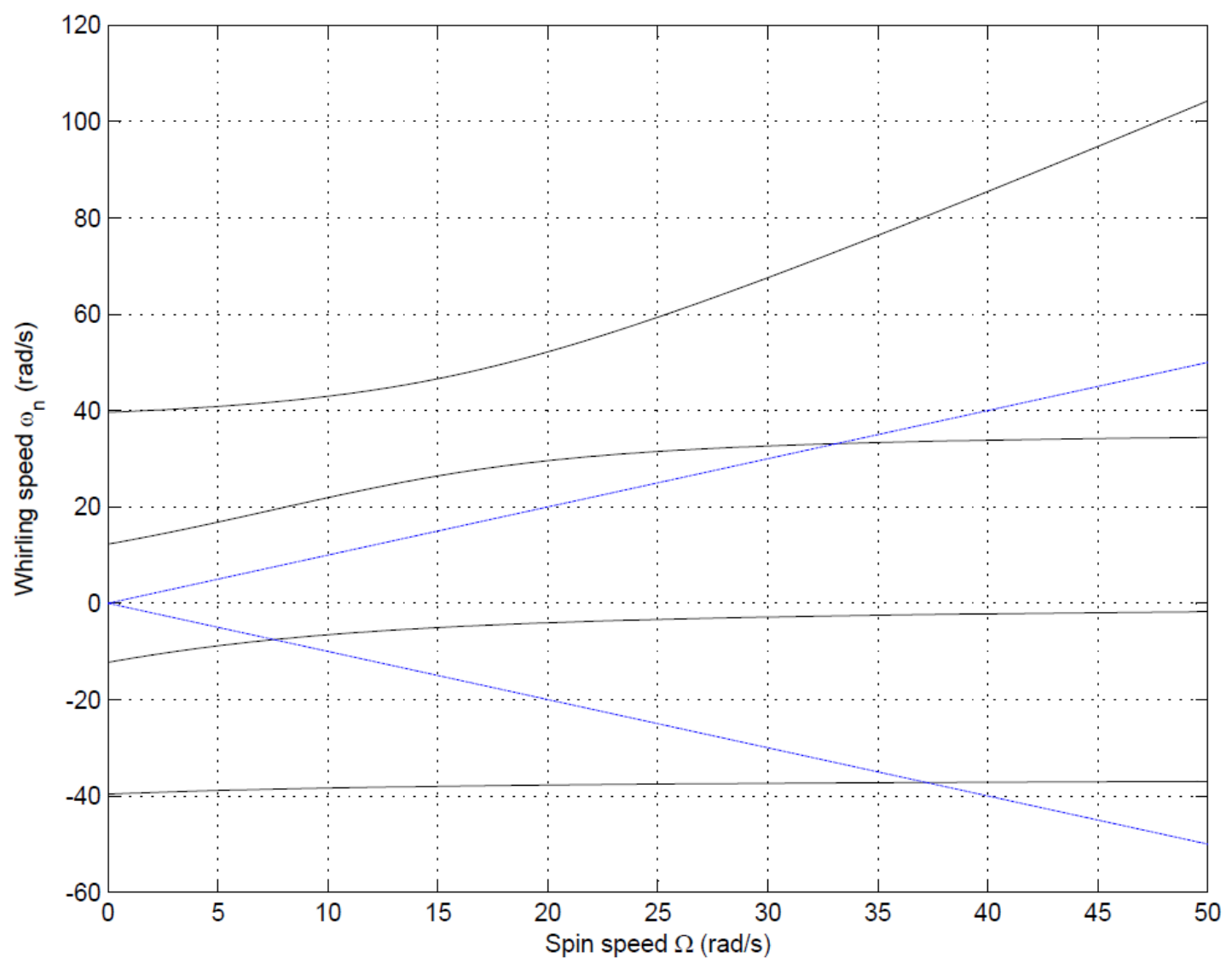

Figure 1.7. Campbell Diagram with rigid bearings and a flexible shaft

Surprisingly, a system with flexible bearings and a flexible shaft has lower critical speeds than a system with a rigid shaft and flexible bearings. The individual critical speeds are $-7.5 \mathrm{rad} / \mathrm{s},-37.2 \mathrm{rad} / \mathrm{s}$ and $33.1 \mathrm{rad} / \mathrm{s}$.

The critical speeds are summarized for all three configuration in Table 1.2. The different configuration are flexible bearings and flexible shaft (FBFS) model, the flexible shaft rigid bearings (FSRB) and the rigid shaft and flexible bearings (RSFB) model. As previously mentioned, the highest critical speeds appear if the shaft is assumed to be rigid but the bearings flexible. It can be seen that the flexibility of the shaft has a greater 
influence on the critical speeds than the flexibility of the bearings. This fact is only valid for this model and can be different with another model or other properties. However, the values of the critical speeds of the two last cases are very close. In a real system, these speeds must be avoided in order to reduce the possibility of resonance.

Table 1.2. Comparison of the critical speeds with different flexibilities

\begin{tabular}{ccc} 
RSFB & FSRB & FSFB \\
\hline $379.60 \mathrm{rad} / \mathrm{s}$ & $33.35 \mathrm{rad} / \mathrm{s}$ & $33.12 \mathrm{rad} / \mathrm{s}$ \\
$-220.52 \mathrm{rad} / \mathrm{s}$ & $-7.55 \mathrm{rad} / \mathrm{s}$ & $-7.55 \mathrm{rad} / \mathrm{s}$ \\
$-427.33 \mathrm{rad} / \mathrm{s}$ & $-37.49 \mathrm{rad} / \mathrm{s}$ & $-37.21 \mathrm{rad} / \mathrm{s}$ \\
\hline
\end{tabular}




\section{Introduction to Wavelet Analysis}

This chapter provides an introduction to Wavelet Transform. In order to understand the importance of its application and the advantage of analyzing non-periodic signals, classical vibrational analysis methods will be explained first. These include the Fast Fourier Transform (FFT), as well as the Short Time Fourier Transform (STFT). The term "fast" is included to the Fourier Transform since the development of a computational algorithm, which is used to process the Fourier Transform more quickly [11].

\subsection{Fourier Transform for Periodic Signals}

Most periodic signals that appear in practical applications or in nature are time domain signals. That means that a variable e.g. a displacement of a shaft is a time dependent variable. However, in many cases it is very important to plot the frequency domain in order to gain more insight of a periodic signal. One way to obtain this information and to transform a signal from its time domain to its frequency domain is to apply the Fourier Transform.

Jean-Baptiste Joseph Fourier ${ }^{1}$ discovered that every periodic signal could be approximated with an infinitive sum of sine and cosine function, which are known as the Fourier series. The Fourier series contains the frequency $f$, which is equivalent to the inverse of the period $T$. Furthermore, the Fourier series includes the coefficients $a_{n}$ and $b_{n}$, which describe the weight of the individual sine and cosine functions. The Fourier series is presented in equation (2.1).

$$
f s(t)=a_{0}+\sum_{n=1}^{\infty} a_{n} \cos \left(\frac{2 \pi n t}{T}\right)+\sum_{n=1}^{\infty} b_{n} \sin \left(\frac{2 \pi n t}{T}\right)
$$

\footnotetext{
${ }^{1}$ French mathematician and physicist (1768-1830)
} 
To illustrate this summing function, a square wave is approximated by the Fourier series in dependence on the number of terms $n$, that are used to carry on and to calculate the summing function. To illustrate that an approximation of a square function is performed in Figure 2.1.Note, the higher the terms of the function are, the better the approximation to the real square function is. A term of $n=1$ shows a simple sine wave. By increasing the number of terms, the square function can almost completely be approximated by the Fourier series. In this example, the error between the real square function and the approximated function is vanishing small when 49 terms are taken to approximate the square function.
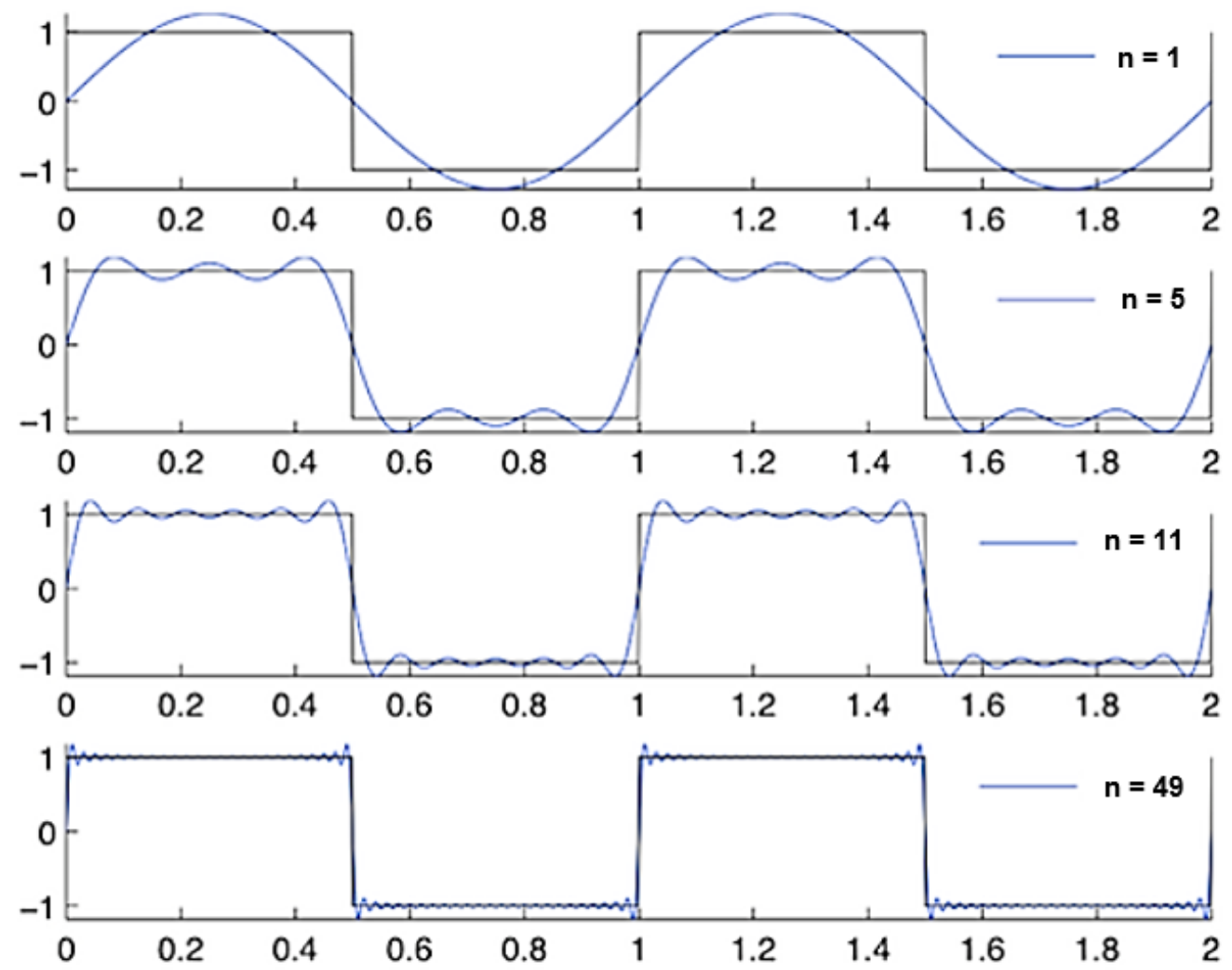

Figure 2.1. Approximation of a square function with a Fourier series [12]

This knowledge reveals that the Fourier series can approximate any periodic function. On the other hand, in many cases it is also important to display the frequency and the 
amplitude of a signal in the frequency domain in order to gain more insight of a function. This can be done using the Fourier transform.

To illustrate this method, a simple sine function with a frequency of $20 \mathrm{~Hz}$ and an amplitude of 0.2 is used as an example. The signal in the time domain is plotted in the upper part of Figure 2.2. Applying the Fast Fourier Transform results in the frequency domain, revealing the correct frequency and the corresponding amplitude.
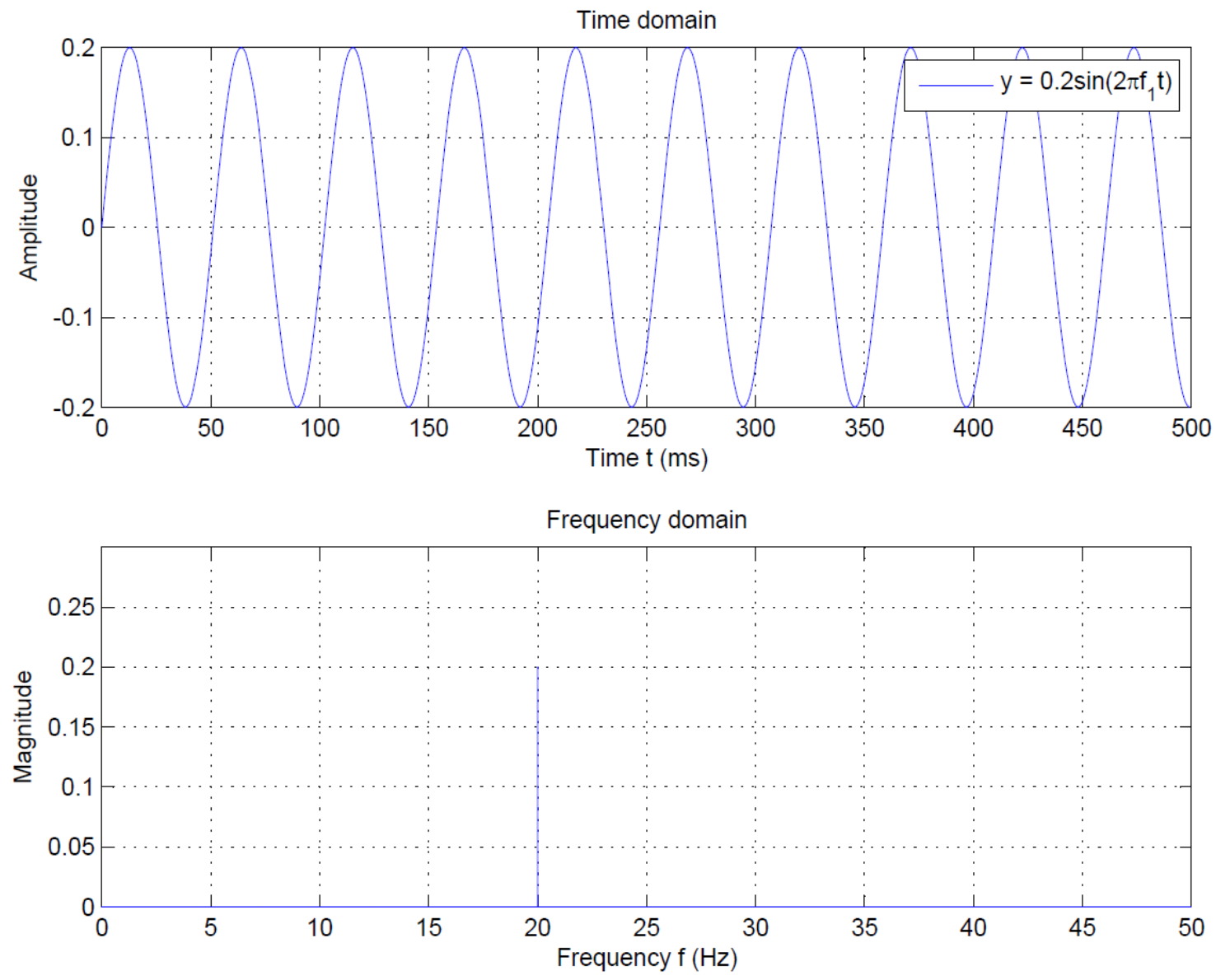

Figure 2.2. Time and frequency domain of a simple sine function

Nevertheless, not only the information of a single sine function can be revealed, nor it is possible to transform any imaginable periodic function from the time domain to its frequency domain. As an example, three different sine functions, each with a different frequency and amplitude, are superimposed and plotted in Figure 2.3. The first sine function has an amplitude of 0.2 and a frequency of $20 \mathrm{~Hz}$, the second superimposed 
sine function contains an amplitude of 0.5 and a frequency of $50 \mathrm{~Hz}$, while the third superimposed function consist of an amplitude of 1 and a frequency of $100 \mathrm{~Hz}$. As you can see, the frequency domain shows the correct frequencies and amplitudes for each superimposed function.
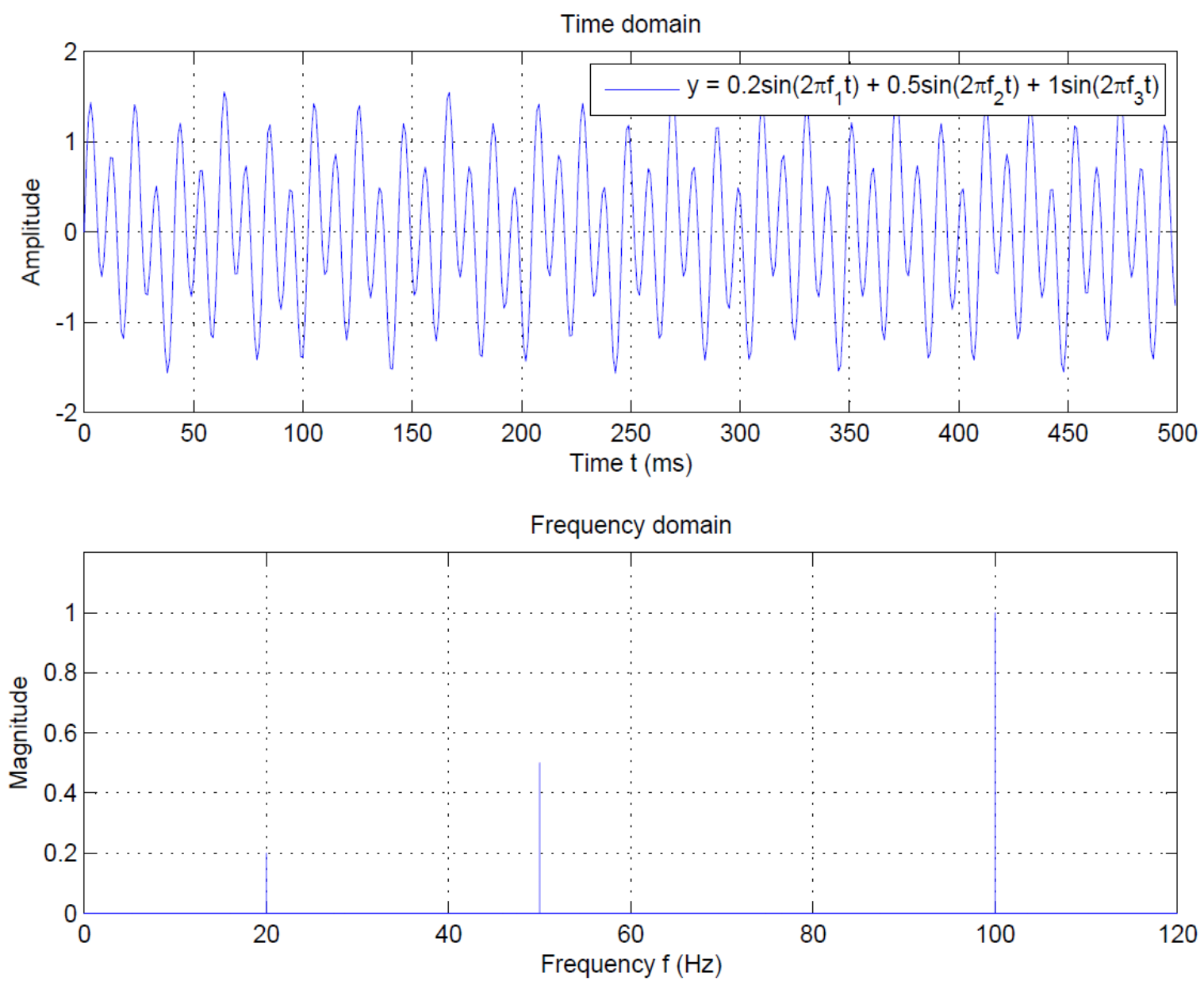

Figure 2.3. Time and frequency domain of a superimposed function

FFT is a powerful tool to determine the frequency and the amplitude of a signal but not suitable for non-periodic functions though. To explain this in detail, the superimposed periodic function from the last example is re-ranged to a non-periodic signal. Rather than being superimposed, the individual function are appearing in different periods of time, shown in Figure 2.4. 
If the FFT is applied to this non-periodic signal, the frequencies still can be somehow identified. However, a clear determination of the amplitude cannot be guaranteed since the frequency domain does not reveal the correct magnitude values. Thus, applying the FFT to a non-periodic signal can result in information losses and should never be used to analyze a non-periodic signal. The phenomenon of information losses is called Leakage and it appears when the signals' energy is reducing over a wide frequency span in the FFT when it should be in a smaller one instead [13].
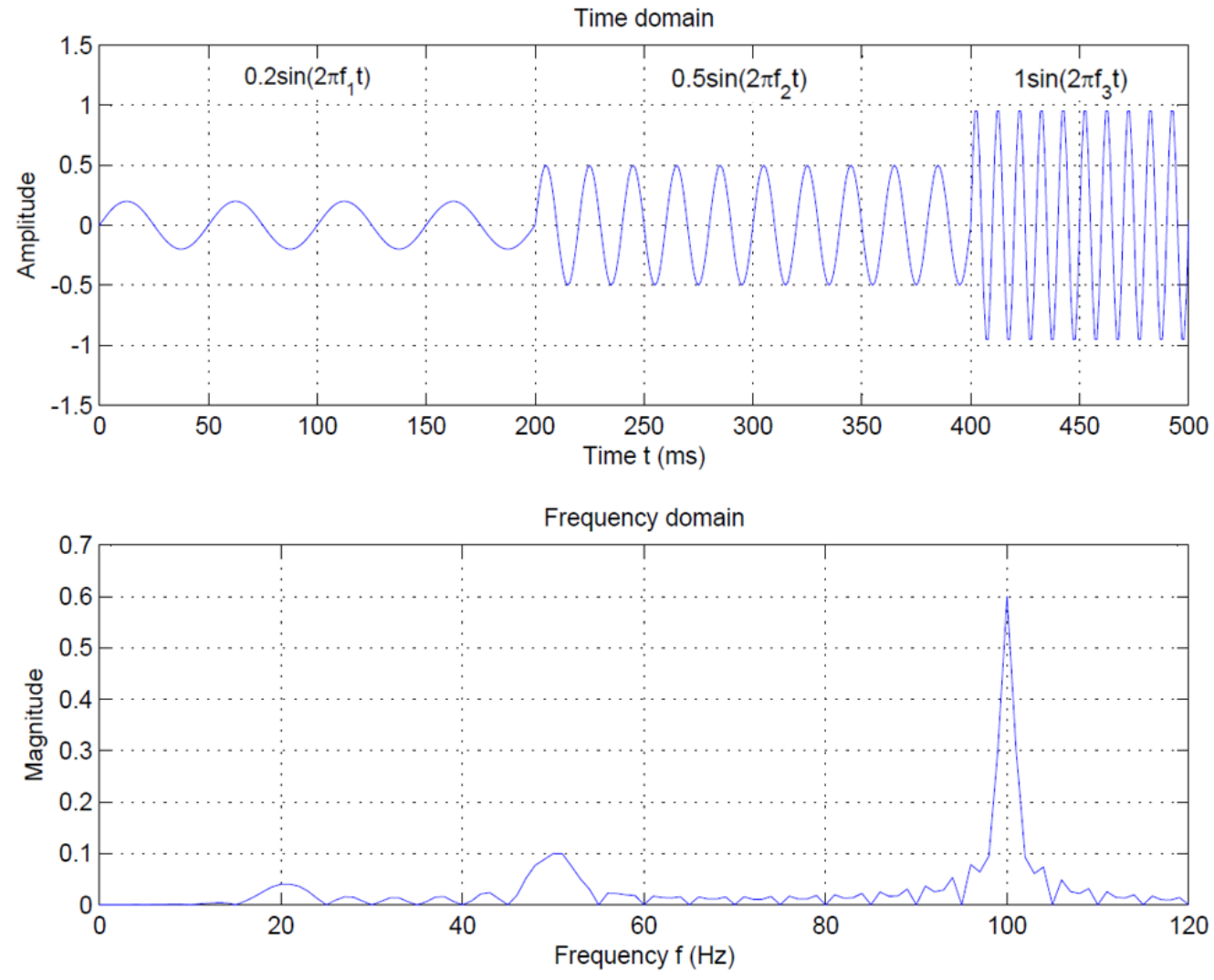

Figure 2.4. Time and frequency domain of a non-periodic function

The fact that FFT does not reveal how a signal is changing over time leads to the consideration of a better method to analyze a non-periodic signal. The Short-Time Fourier Transform, for instance, is a better method to analyze non-periodic functions, which is the subject of the next section. 


\subsection{The Short Time Fourier Transform (STFT)}

A better solution to overcome the drawback of the FFT is to introduce a method, which divides the signal into thin slices and performs a Fourier Transform for each slice.

This means that tiny time slices (windows) of the signal are taken and the FFT of every slice is performed in order to create FFT plot over the signals' time range. The frequency spectrum appears now as a three-dimensional plot with an additional time-axis.

The method of the STFT performs for each time slice a Fourier Transform. Thus, a constant window is used for each time slice and then shifted to the next time slice along the time line axis, where again another Fourier Transform is performed [14].

However, there are plenty of window functions available to perform the FT. A sample of windows is presented in Figure 2.5. Each window function has its own characteristics, coming along with its own disadvantages and advantages. Thus, the window function strongly depends on the signal that is to analyze. 


\begin{tabular}{|l|l|l|l|l|}
\hline \multicolumn{1}{|c|}{ Window } & $\begin{array}{l}\text { Best for these } \\
\text { Signal Types }\end{array}$ & \multicolumn{1}{|c|}{$\begin{array}{c}\text { Frequency } \\
\text { Resolution }\end{array}$} & $\begin{array}{r}\text { Spectral } \\
\text { Leakage }\end{array}$ & \multicolumn{1}{|c|}{$\begin{array}{c}\text { Amplitude } \\
\text { Accuracy }\end{array}$} \\
\hline Barlett & Random & Good & Fair & Fair \\
\hline Blackman & $\begin{array}{l}\text { Random or } \\
\text { mixed }\end{array}$ & Poor & Best & Good \\
\hline Flat top & Sinusoids & Poor & Good & Best \\
\hline Hanning & Random & Good & Good & Fair \\
\hline Hamming & Random & Good & Fair & Fair \\
\hline Kaiser-Bessel & Random & Fair & Good & Good \\
\hline None [boxcar] & $\begin{array}{l}\text { Transient \& } \\
\text { Synchronous } \\
\text { Sampling }\end{array}$ & Best & Poor & Poor \\
\hline Tukey & Random & Good & Poor & Poor \\
\hline Welch & Random & Good & Good & Fair \\
\hline
\end{tabular}

Time Domain Window Shapes
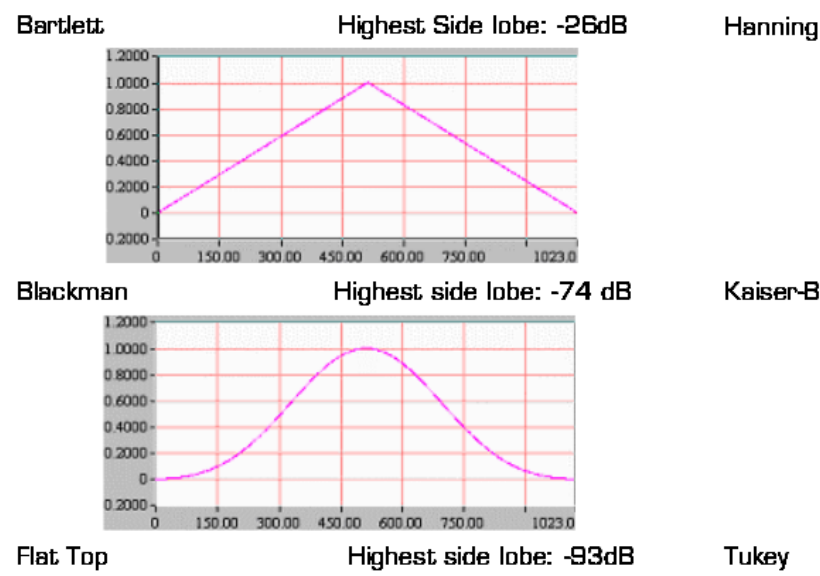

Highest side lobe: $-32 \mathrm{~dB}$

Figure 2.5. Different window functions and their properties [13]

The selection of a window cannot be arbitrary chosen and therefore depends on the type of signal that is analyzed. Furthermore, several disadvantages have to be taken into account when a window function is considered for a frequency analysis. Wide windows, for instance, can result in an excellent frequency resolution but the time resolution can get worse. On the other hand, if a narrow window size is chosen, the time resolution can get better but the frequency resolution will worsen instead [15].

The problem is a result of choosing a window function that is suitable for the entire signal range and that has a compromise for both - a good time resolution and a good frequency resolution [15]. However, this can be very difficult, especially when frequencies are changing over time. 
This example means that it is not always easy, especially if the exact form of the signal is unknown, to find the right window size in order to get all necessary information from the 3D FFT plot. This drawback, as well as the exact determination of an appropriate overlapping size, which is not further explained in this report, can lead to information losses and arises the need of a more suitable and better method. This is where Wavelet Transform comes into consideration and is explained in the next section.

\subsection{The Wavelet Transform (WT)}

The Wavelet Transform was created as another option in order to solve the resolution problem that occurs when applying the STFT. The Wavelet analysis is done in the similar way to the STFT, but major differences between these two methods exist. In contrast to the STFT, where the window size is fixed and therefore cannot be changed, the Wavelet Transform allows the window size to be variable. This fact leads to a detection of different frequencies over the whole signals' time range. Furthermore, it fixes the resolution problem.

This is realized with a special set of scaling functions that every Wavelet function includes and enables a scaling and shifting of the window over the time range. Only with this method, it is possible to detect all necessary information of a signal.

The Wavelet formulation is presented in equation (2.2). Where $s$ stands for the scale and is related to the actual frequency of a signal. Furthermore, the mother wavelet $\psi$ which is the transforming function depends on the time shift $\tau$ and the time $t$ of the signal $x(t)$.

$$
\Psi_{x}=\frac{1}{\sqrt{s}} \int x(t) \psi\left(\frac{t-\tau}{s}\right) d t
$$


The parameter scale enables a detailed analysis of a non-periodic signal. In this case, low frequencies correspondent to high scales. Vice versa, high frequencies correspondent to low scales. Thus, Wavelet analysis allows a high-resolution detection of low and high frequencies.

Similar to FFT or STFT with windows, the Wavelet Transform has a variety of Wavelet functions available, each containing a corresponding scaling function. A Wavelet function with its scaling function from the Daubechies family is presented in Figure 2.6.
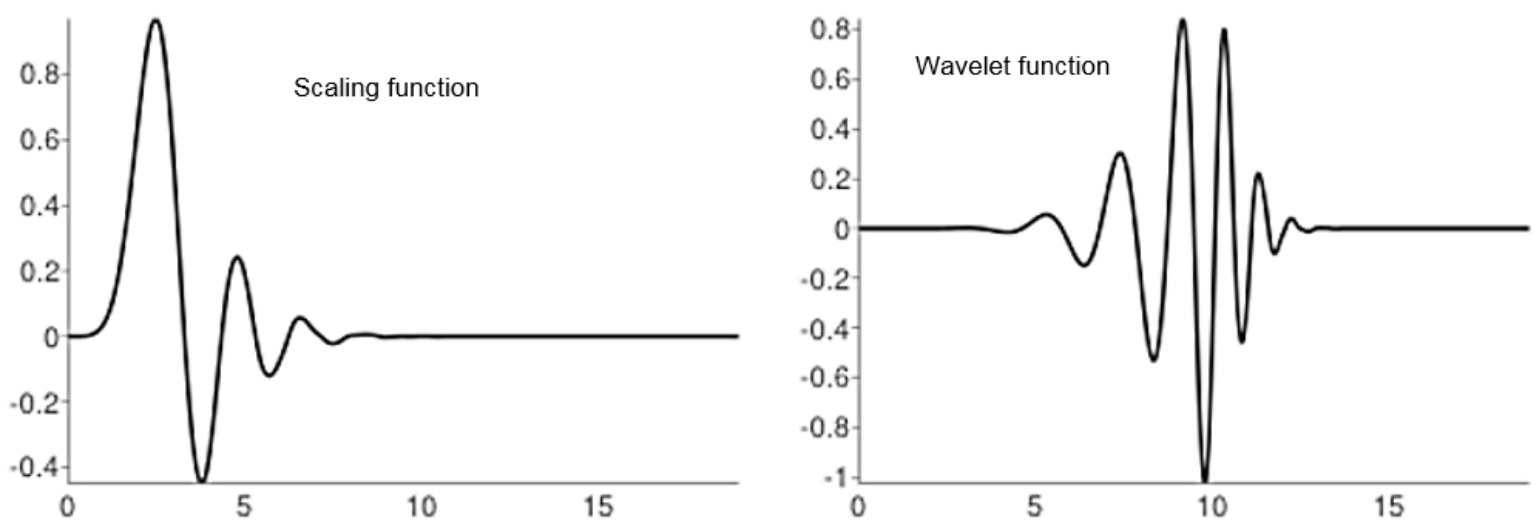

Figure 2.6. Wavelet function and the corresponding scaling function [16]

In order to demonstrate the effectiveness of the Wavelet Transform several example will be used. The first example shows simple sine wave function, with an amplitude of 0.2 and a frequency of $20 \mathrm{~Hz}$, which is the same function as the previous example plotted in Figure 2.2.

Applying the Wavelet Transform of this sine signal leads to the scalogram in Figure 2.7. The result is presented in terms of scales and time or space. The term space stands for the number of samples of a signal. The 1D Continuous Wavelet Transform requires only one variable of a signal (e.g. the displacement) and takes its number of samples to process the WT. The number of samples is related to the time and therefore 
named space or time. In this specific example, the number of samples matches with the time in $\mathrm{ms}$.

The scales of the WT are plotted on the vertical axis, which are related to the frequencies. The correspondence of the frequencies and the scales will be explained in more detail later in this chapter.

Another important parameter in the scalogram is the percentage of energy wavelet coefficient. This parameter reveals the maxima of energy in the scalogram, which is similar to the peaks in a 3D FFT plot.

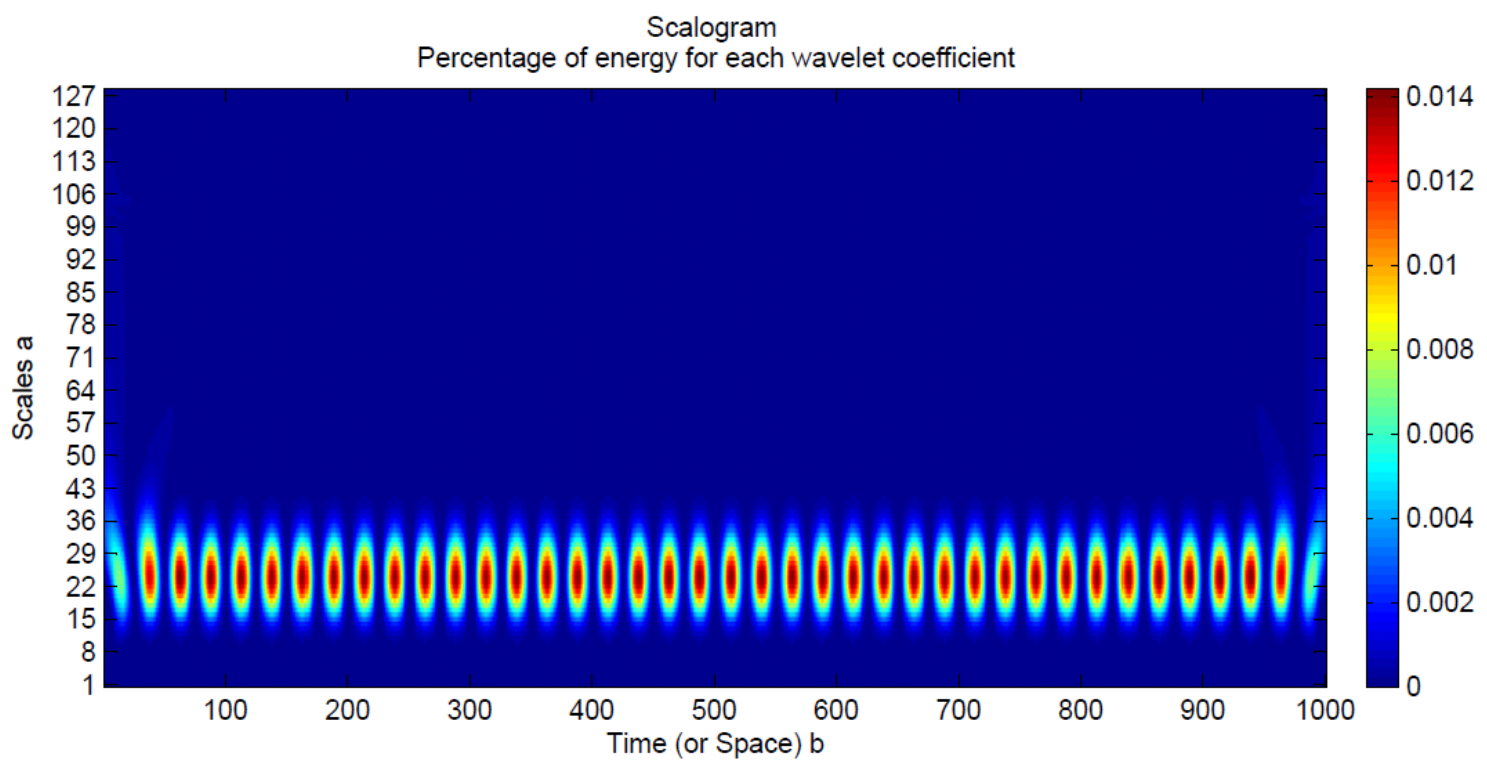

Figure 2.7. Wavelet plot of a simple sine wave function (created with [17])

In Figure 2.7, the sine wave shows an energy maximum at a scale of about 25 . In order to obtain the frequency value at this specific scale, the scales have to be transferred back to the corresponding frequencies. This is realized in Figure 2.8 where the corresponding values of the scales and frequencies are plotted. As you can see, a scale of 25 corresponds to a frequency of $20 \mathrm{~Hz}$. This is exactly the frequency of the sine wave that is used in this example. 


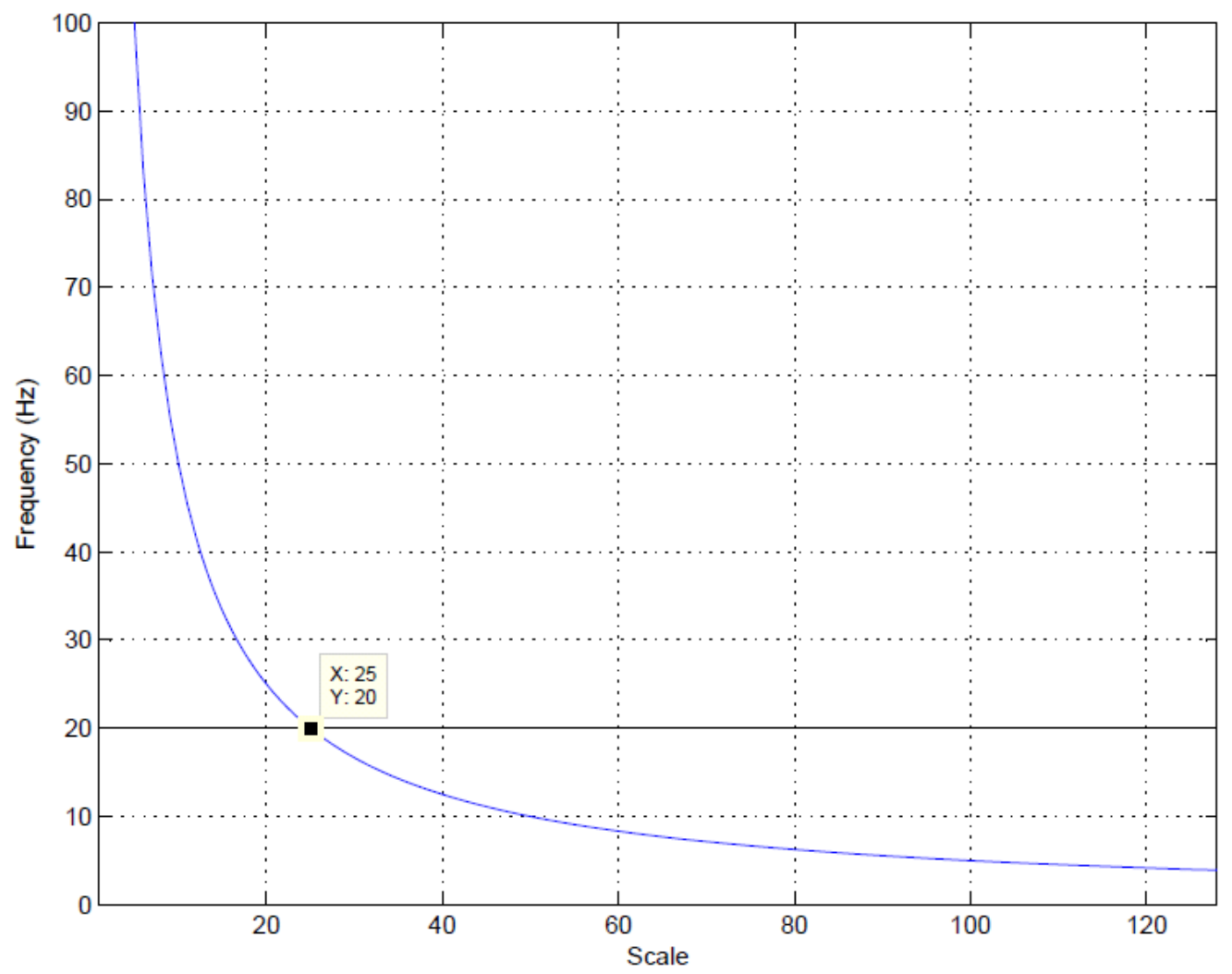

Figure 2.8. Corresponding scales and frequencies of the WT (created with [17])

Similar to the FFT, WT can also detect the frequencies of a superimposed function. The superimposed function consist of two sine waves, each with a different frequency $(20 \mathrm{~Hz}$ and $200 \mathrm{~Hz})$. This function is presented in equation (2.3).

$$
x(t)=\sin (2 \pi 20 t)+\sin (2 \pi 100 t)
$$

The corresponding Wavelet Transform of this function is plotted in Figure 2.9. As you can see, two energy maxima appear in the scalogram, which describe the frequencies of the system. These energy maxima appear at a scale of around 6 and 25 . Using the corresponding frequency and scale graph in Figure 2.8, the frequencies of $100 \mathrm{~Hz}$ for a scale of 6 and of $20 \mathrm{~Hz}$ for the scale of 25 can be revealed. 

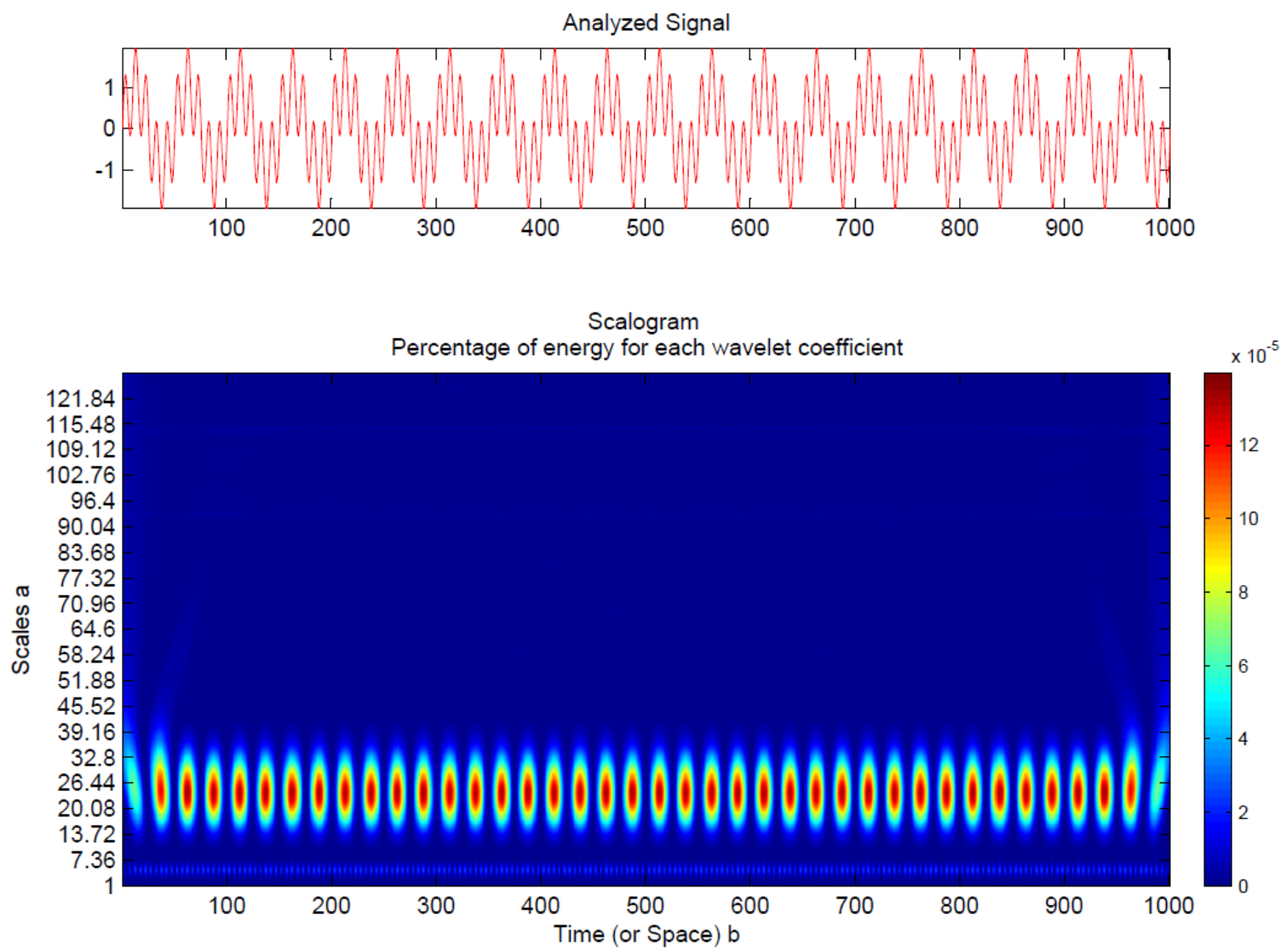

Figure 2.9. Wavelet plot of a superimposed sine function (created with [17])

However, the presented example is still a periodic superimposed function and could be analyzed using the FFT or STFT to obtain the frequencies. In order to demonstrate a meaningful advantage of the WT, the sine function of the last example will be reranged to a non-periodic signal. Now, the sine wave with a frequency of $20 \mathrm{~Hz}$ appears in the time range between 0 and $250 \mathrm{~ms}$ and again between 750 and $1000 \mathrm{~ms}$. The other $100 \mathrm{~Hz}$-sine function is present in the time range between $250-750 \mathrm{~ms}$.

The analyzed signal, as well as the Wavelet result, is presented in Figure 2.10. As you can see, the energy maxima appear at a scale of 25 between 0 and $250 \mathrm{~ms}$ and again between 750 and $1000 \mathrm{~ms}$. In addition, energy maxima are present at a scale of six between 250 and $750 \mathrm{~ms}$. Thus, Wavelet is a suitable method to analyze non-periodic and non-stationary signals with a high resolution for both, the frequency and the time. 

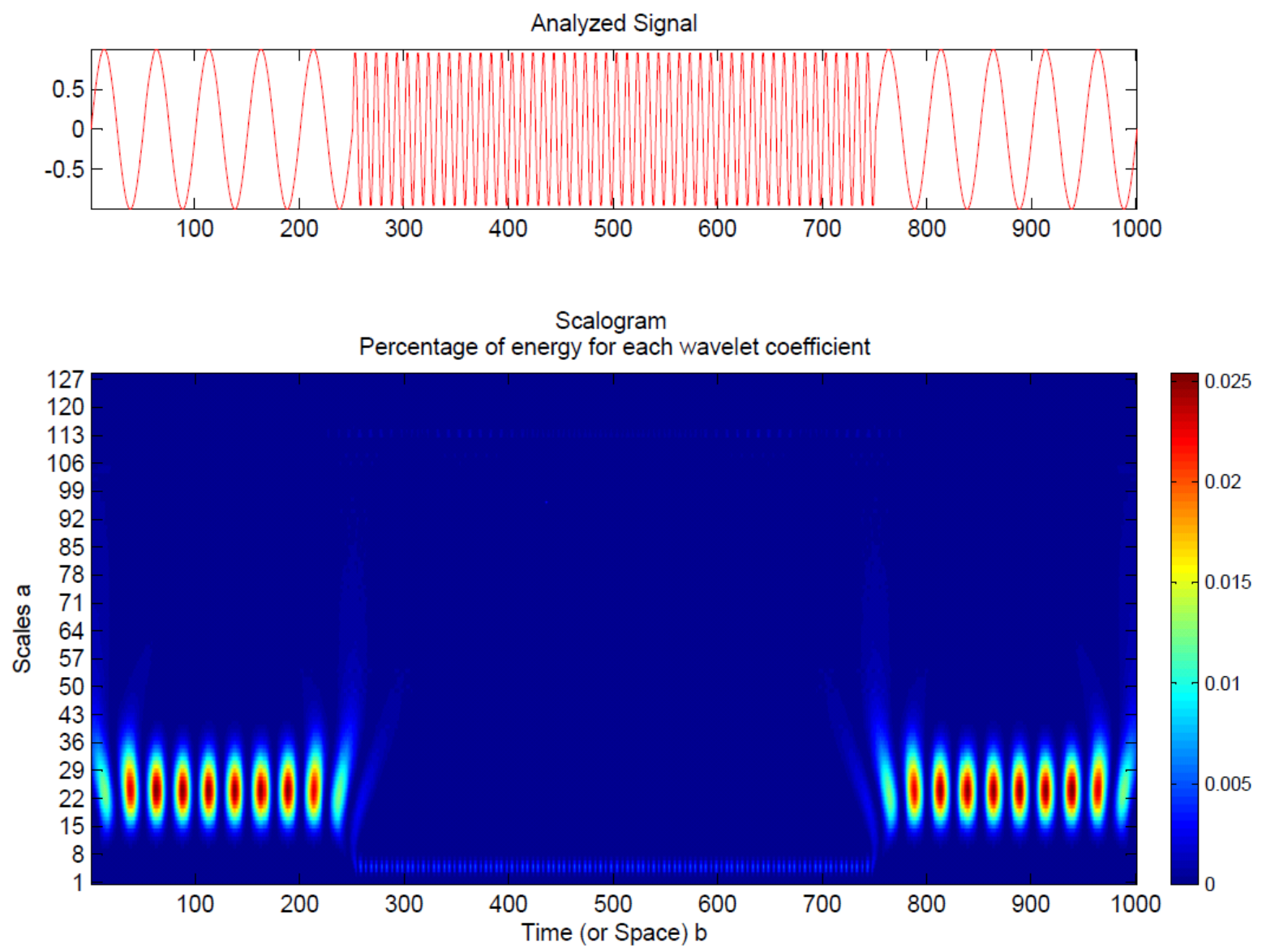

Figure 2.10. Wavelet plot of a non-periodic function (created with [17])

The Wavelet Transform does not only allows the analysis of a pure signal; it also enables the study of any signal, even if noise is present. Signals obtained in practice usually contain noise. In order to perform a vibrational analysis, noise can be an interference factor and can lead to difficulties when performing a vibrational analysis. In many cases, filters are used in order to reduce the presence of the noise and to clarify the signal. However, another example will be used to demonstrate that it is possible to get the right frequencies with Wavelet Transform, even if a signal contains a lot of noise.

The same superimposed sine function, which was used in equation (2.3), will be the objective for the next analysis. Furthermore, a random noise function will be added to 
this function to get a more realistic signal that can occur in any practical application. Figure 2.11 shows the Wavelet plot of the new signal.

Even the signal is noisy and the energy maxima are not clearly formed, the scales and respectively the frequencies of the signal can be identified. Again, the Wavelet plot reveals that the energy maxima occur at a scale of 25 and 6 . These two values correspond to the frequency of $20 \mathrm{~Hz}$ and $100 \mathrm{~Hz}$. Therefore, it is demonstrated that a signals' frequencies can be revealed even if noise is present using WT.
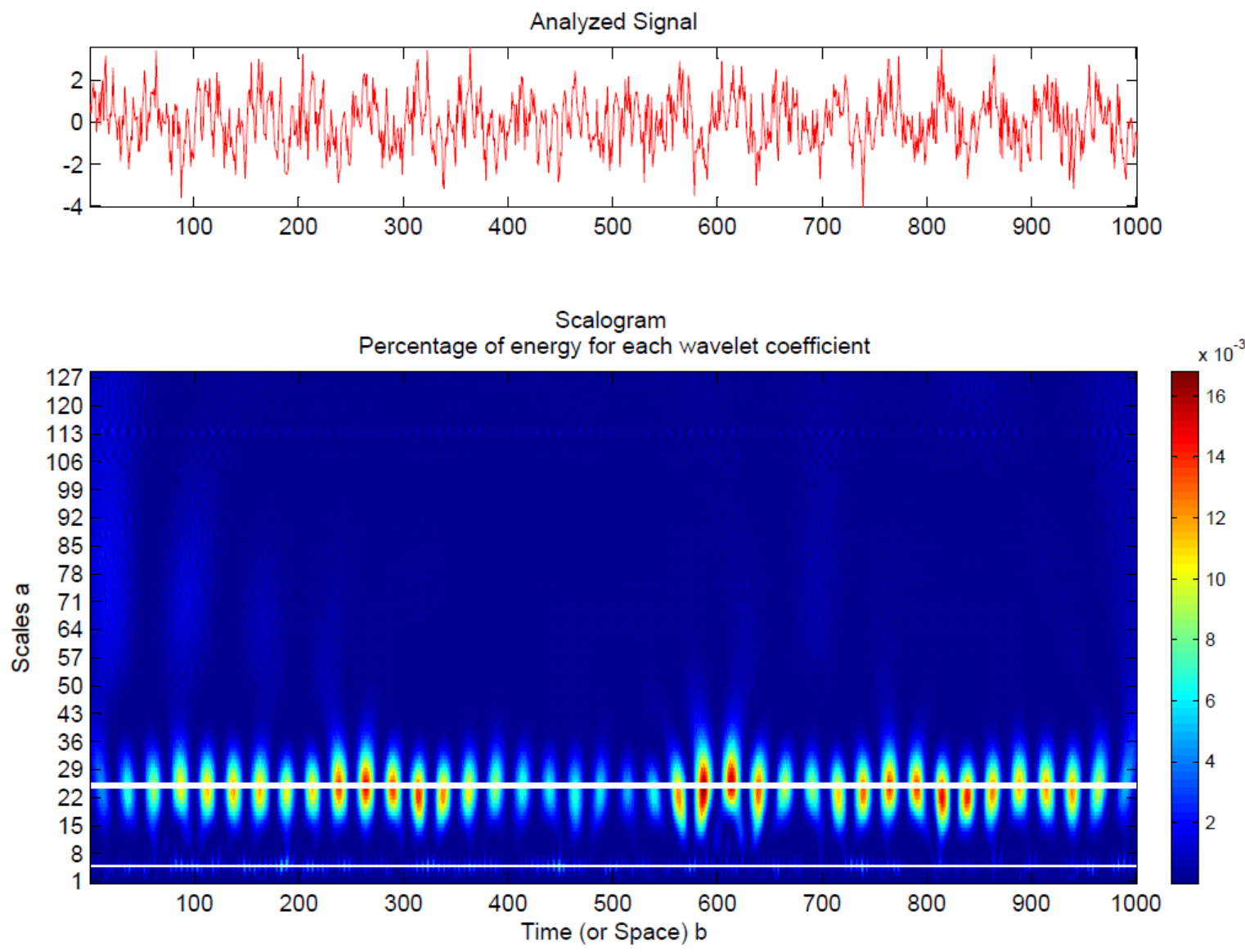

Figure 2.11. Wavelet plot of a superimposed sine function with noise (created with [17]) 


\subsection{Summary}

The Wavelet Transform was demonstrated starting from explaining the Fourier Transform. The FFT is a suitable tool to perform vibration studies of periodic signals. However, performing a FFT to non-periodic signals can lead to leakage, which can turn into information losses. A solution can be the Short-Time Fourier Transform (STFT), with which the frequency spectrum can be presented as a 3D frequency plot, where an additional time axis is added to the frequency spectrum. Even STFT is a powerful tool to analyze non-periodic signals, the settings of the window and the determination of the window itself is very important, since it can lead to the resolution problem. To overcome this drawback the Wavelet Transform was introduced to perform vibrational analysis. It is shown that the WT is suitable to analyze a non-periodic signal and to obtain simultaneously a multi-resolution result, which makes the WT a highly powerful tool. Furthermore, it was demonstrated that even a noisy signal can be analyzed using WT. 


\section{Simulation of the Gyroscopic Effect in Adams}

\subsection{Introduction}

This chapter contains the analysis of the gyroscopic effect using the multibody dynamics software MSC Adams. The research and analysis of the gyroscopic effect of a single shaft-disk system is split up into several sections.

Several parameters like the bearing stiffness and the location of the disk are changed and the systems' response is measured. The shaft of the rotor system is modeled using the FEA software Abaqus. In order to create an exportable MNF model, a script file is used to convert the FEA model into a Modal Neutral File, which is then imported in Adams. The bearings of the system are modeled using the features bushings. This bushing model allows a convenient change of parameters such as the bearing stiffness. Furthermore, it allows the reduction of the simulation time.

The output of the system is measured in terms of the Euler Angle that represent the angular displacement at the location of the disk. The Euler Angle are a clear and unique method to determine the presence of the gyroscopic effect in a rotating system. In addition, the orbit plot of the disk is plotted and analyzed.

Another analysis includes the effect of eccentricities in a shaft-disk system. In order to create the eccentricities, different masses are placed onto the rotor disk. The FFT analysis is used to compare the effect of the vibration with different eccentricities.

Furthermore, an anisotropic bearing system will be created and the orbit plot of the shaft will be drawn. 


\subsection{Variation of the Bearing Stiffness}

\subsubsection{Model Setup}

The first simulation includes the analysis of the Euler Angle in dependence of the bearing stiffness. To model the gyroscopic effect, a single shaft-disk model needs to be created in Adams. A sketch of the rotor model is presented in Figure 3.1. The dimension and the properties of this system are shown in Table 3.1. Except for the bearing stiffness, which is a variable parameter in this study, all other parameters remain unchanged during each simulation.

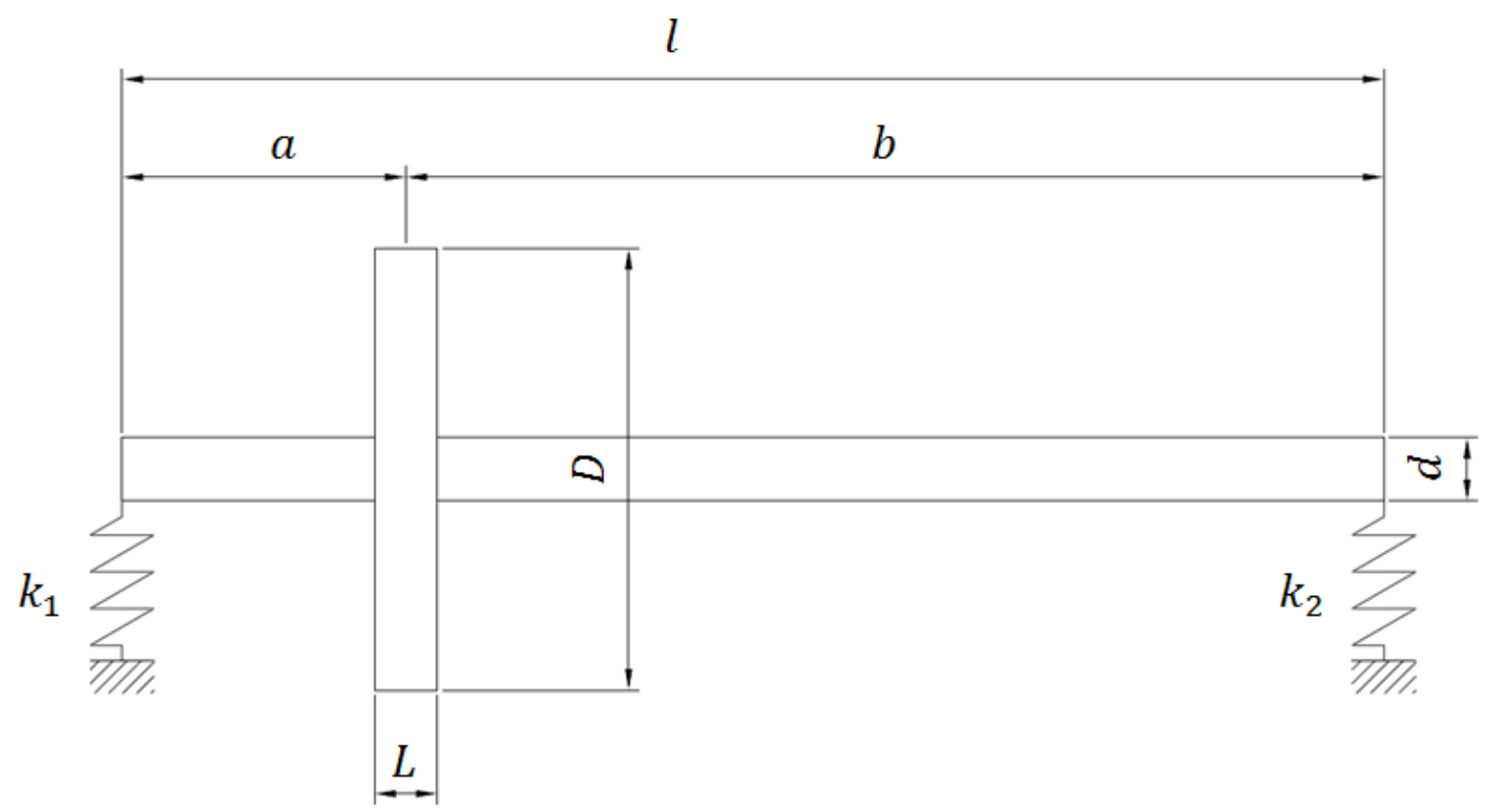

Figure 3.1. Single shaft-disk model

The shaft of the model has a length of $0.5 \mathrm{~m}$ and a diameter of $0.01 \mathrm{~m}$. The disk is mounted at $0.125 \mathrm{~m}$ measuring from the left end of the shaft and has a diameter of $0.15 \mathrm{~m}$. The properties of both, the disk and the shaft, are assigned with the material properties of steel. While the disk is modeled as a rigid body, the shaft is created as a flexible body. 
Table 3.1. Parameter and properties of the shaft-disk model

\begin{tabular}{cccc} 
Symbol & Parameter name & Value & Unit \\
\hline$a$ & Disk location & 0.125 & $\mathrm{~m}$ \\
$D$ & Diameter of disk & 0.150 & $\mathrm{~m}$ \\
$d$ & Diameter of shaft & 0.01 & $\mathrm{~m}$ \\
$E$ & Modulus of elasticity & $2.07 \mathrm{E}+11$ & $\mathrm{~N} / \mathrm{m}^{2}$ \\
$L$ & Disk length & 0.02 & $\mathrm{~m}$ \\
$l$ & Shaft length & 0.5 & $\mathrm{~m}$ \\
$k_{1}$ & Stiffness of Bearing 1 & variable & $\mathrm{N} / \mathrm{m}$ \\
$k_{2}$ & Stiffness of Bearing 2 & variable & $\mathrm{N} / \mathrm{m}$ \\
$\rho$ & Density & 7,800 & $\mathrm{~kg} / \mathrm{m}^{3}$ \\
$v$ & Poisson ratio & 0.29 & - \\
\hline
\end{tabular}

The values that are used are chosen arbitrarily and will be used as standard values for other experiments in this paper. The stiffness components of the bearings, which are varied during the tests, are shown in Table 3.2. In total, for this study, seven different simulation experiments are performed, each with an individual stiffness value. The stiffness is reduced in every experiment by a factor of 10 , starting from a value from $10,000,000,000 \mathrm{~N} / \mathrm{m}$. Note that the stiffness value of $10,000,000 \mathrm{~N} / \mathrm{m}$ in test procedure number 4 is used as a standard stiffness value for other analyses.

Table 3.2. Stiffness values for each simulation

\begin{tabular}{cc} 
Simulation number & Stiffness $\mathbf{( N / m )}$ \\
\hline 1 & $10,000,000,000$ \\
2 & $1,000,000,000$ \\
3 & $100,000,000$ \\
4 & $10,000,000$ \\
5 & $1,000,000$ \\
6 & 100,000 \\
7 & 10,000 \\
\hline
\end{tabular}




\subsubsection{Model in Adams}

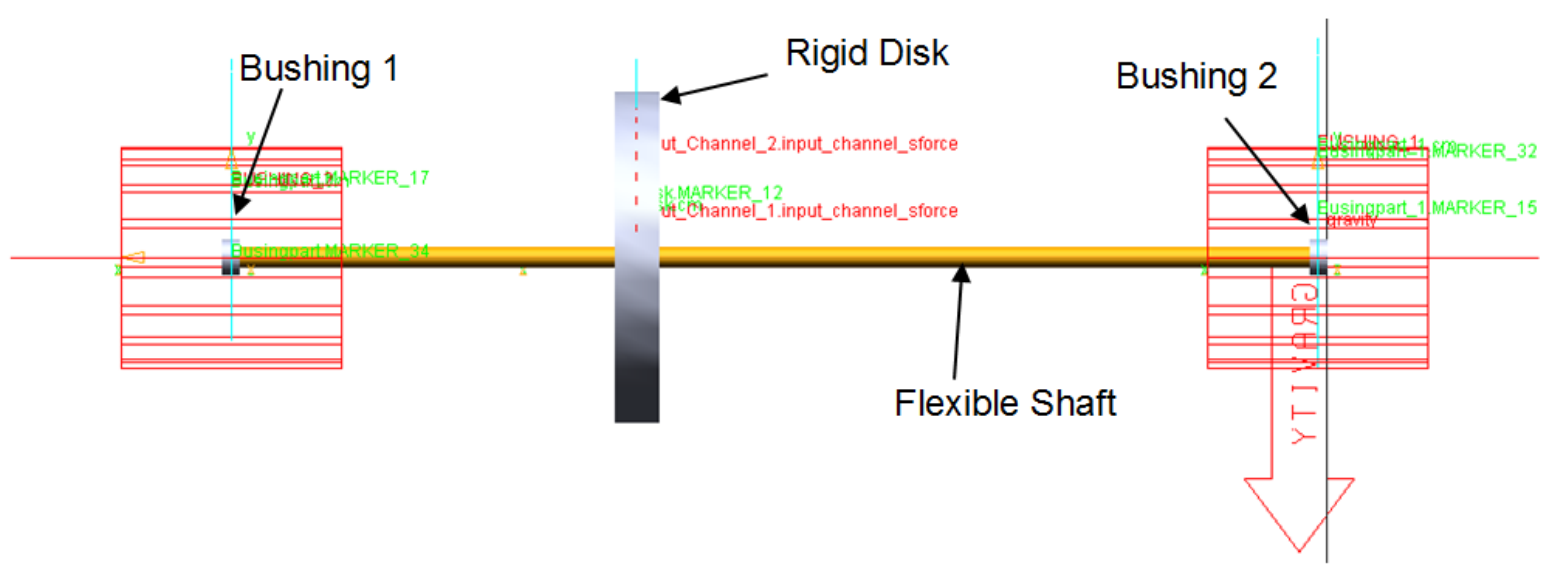

Figure 3.2. Adams model of the single shaft-disk system

Figure 3.2 shows the Adams model of the shaft-disk system. It consist of a rigid rotor disk mounted on a flexible shaft. The shaft is supported by bushings on each ends. The shaft, as previously mentioned, is a flexible body with a mesh size of $1 \mathrm{~mm}$. See the appendix how to create a flexible body in Abaqus and how to import it in Adams.

Furthermore, instead of using the bearings option in Adams, bushings are used. The reason for that is that the bearings in Adams do not support any changes of the properties like the stiffness or the damping. It is also difficult to verify and evaluate how accurate the bearing option in Adams is and therefore bushings are used.

With the bushings option it is possible to analyze the system, depending on different dynamic properties such as the stiffness or the damping. Therefore, the bushing option is a convenient tool to create pseudo-bearings. Figure 3.3 shows the available bushings option with the option to specify different bearing properties.

Besides changing the stiffness components, it is also possible to change the damping components. Moreover, the property manager contains rotational and translational parameter of these properties. 
This available set up makes the bushing option a powerful tool for dynamic studies. However, they do not represent a real bearing system, since the stiffness of bearings can include a speed dependency.

However, with the use of bushing the simulation time can significantly be reduced. For instance, in this research, the simulation time was reduced up to $98 \%$, when bushings were used instead of bearings. In particular, the simulation was carried out with a total number of 150,000 steps and a simulation time of 15 seconds. The simulation took about two minutes, while with the usage of the bearing option the simulation duration exceeded two hours.

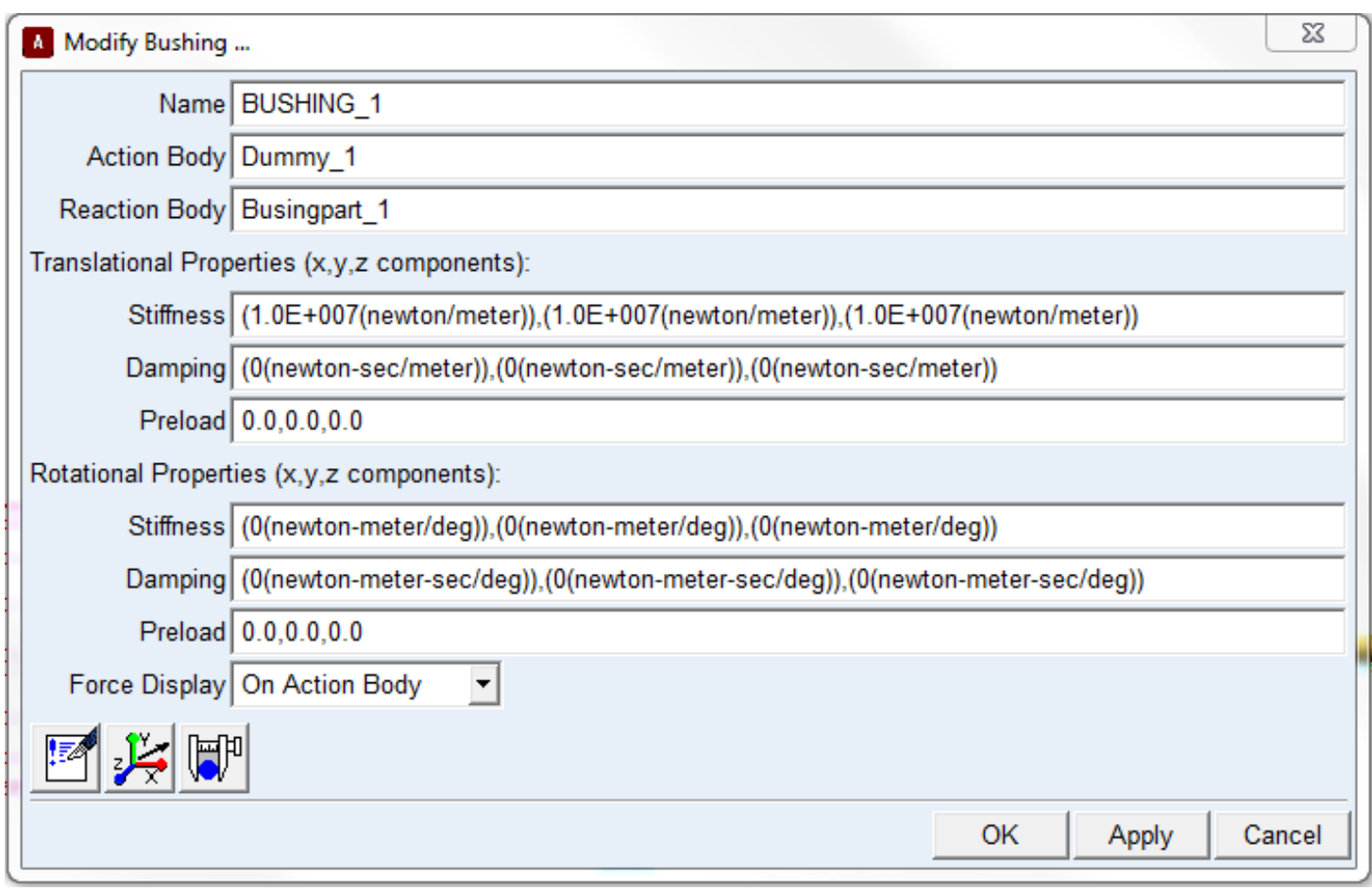

Figure 3.3. Bushing property manager in MSC Adams

Furthermore, a constant speed is applied to the shaft of the system. The speed is hold constant at a value of $15 \mathrm{~Hz}(900 \mathrm{rpm})$. 


\subsubsection{Results}

The result of the gyroscopic effect is presented in terms of the yawing and pitching motion of the disk. The yawing, as well as the pitching, can be described by the Euler Angles of rotation, which are a result of the integrated motion around the disk.

The Euler Angle around the (horizontal) $x$-axis describes the pitching motion of the disk, while the Euler Angle around the (vertical) y-axis describes the yawing motion of the disk. Figure 3.4, as well as Figure 3.5, shows the simulation results of the pitching and yawing motion with different stiffness values.

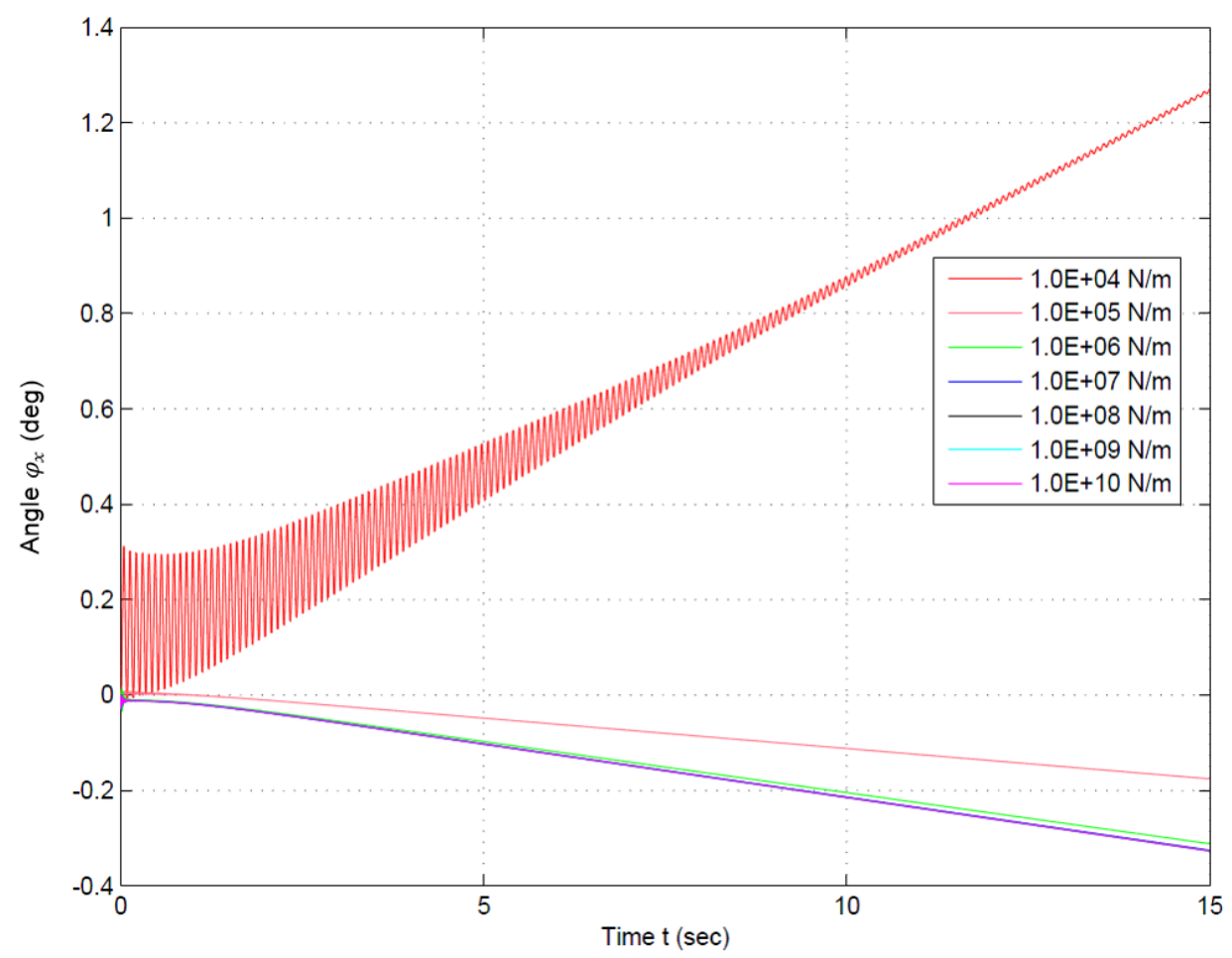

Figure 3.4. Euler Angle (pitching) with different bearing stiffness values

As you can see, several graphs of the pitching angle exist, each representing a different bearing stiffness. The form of the graphs show a linear function, which is due to the integrated constant motion around the disk. In this example, a constant speed around the z-axis is applied to the shaft, which creates a constant precession motion 
around the disk. The integration of this motion results in a linear angular displacement function.

Figure 3.5 shows that once the stiffness exceeds a value of $1.0 \mathrm{E}+06 \mathrm{~N} / \mathrm{m}$, the influence on the pitching becomes less important, which means that the differences between the individual graphs are small and the changes are minimal. This can be seen in detail in Figure 3.5 where the graphs of the pitching motion are shown in a range between 1.0E $+06 \mathrm{~N} / \mathrm{m}$ and $1.0 \mathrm{E}+10 \mathrm{~N} / \mathrm{m}$ as a close-up image. As you can see, the individual graphs are slightly apart and show only a small difference in the Euler Angle.

Notice that the graph with the lowest stiffness value of $1.0 \mathrm{E}+04 \mathrm{~N} / \mathrm{m}$ is present in the opposite direction than the other graphs, which means that for this particular stiffness, the pitching appears in the opposite direction. The bearing stiffness of $1.0 \mathrm{E}+04 \mathrm{~N} / \mathrm{m}$ shows some instabilities since the pitching motion does not reach steady state.

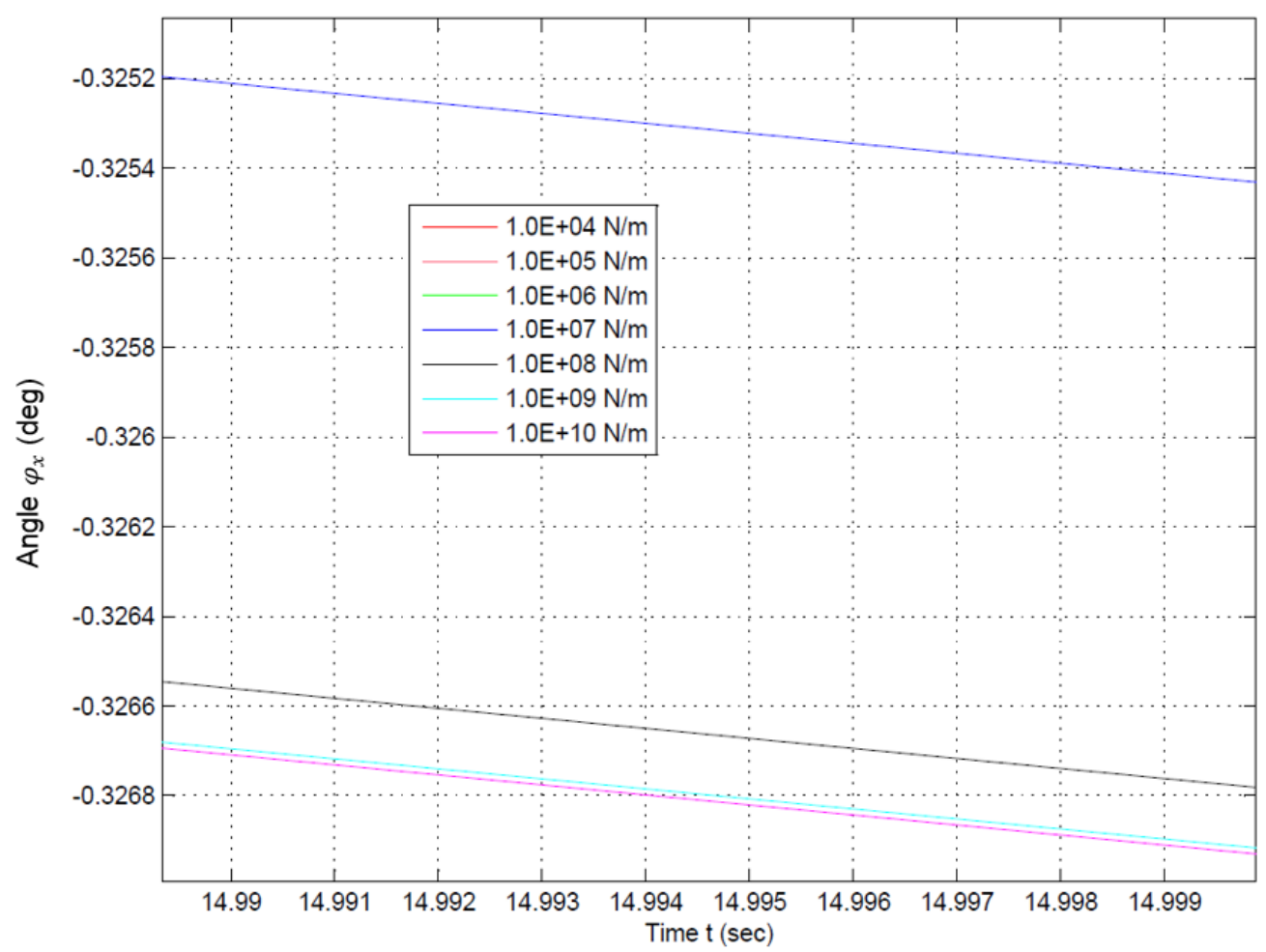

Figure 3.5. Euler Angle (pitching) with different bearing stiffness values (zoom) 
It is also important to analyze the yawing motion of the disk, since the yawing and pitching results in the presence of the gyroscopic effect. Therefore, the yawing motion is described by the Euler Angle $\varphi_{y}$ around the vertical axis. Figure 3.6 shows the simulation result of this angle in dependence of different stiffness values.

Some similarities exist if the yawing and pitching are compared. For instance, the yawing motion increases with the bearing stiffness. In addition, once the stiffness exceeds a value of $1.0 \mathrm{E}+07 \mathrm{~N} / \mathrm{m}$ the differences between the individual graphs become very small and the bearing stiffness becomes less important to the influence of the gyroscopic effect, since the bearings become rigid and the flexibility of the shaft accounts for the GE.

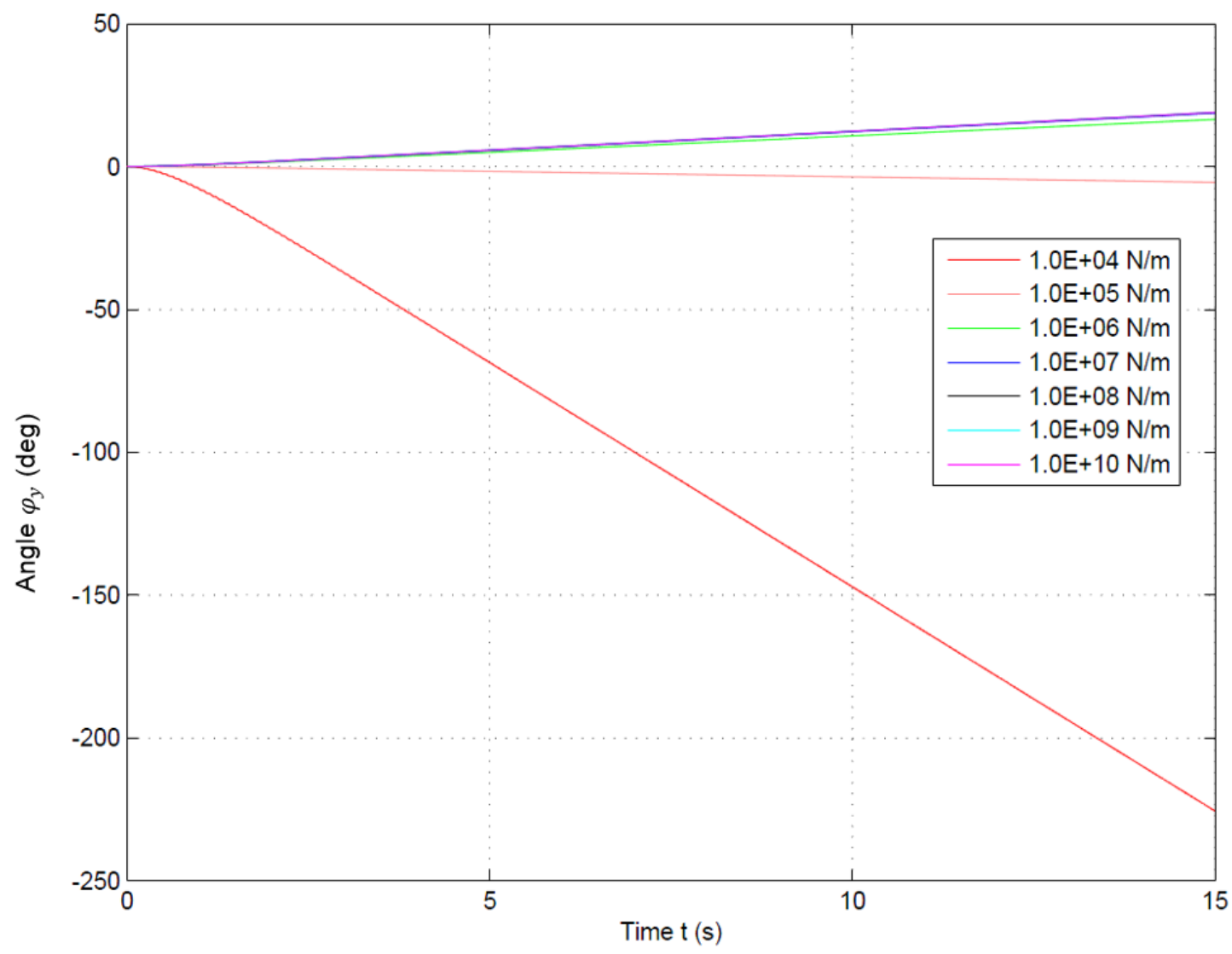

Figure 3.6. Euler Angle (yawing) with different bearing stiffness values 
Notice that the yawing motion is in general higher than the pitching motion. For instance at the end of the simulation time, the yawing angle reaches a value between 18 and 19 deg for high stiffness values. The pitching, on the contrary, reaches a value of about -0.3 deg for high stiffness values.

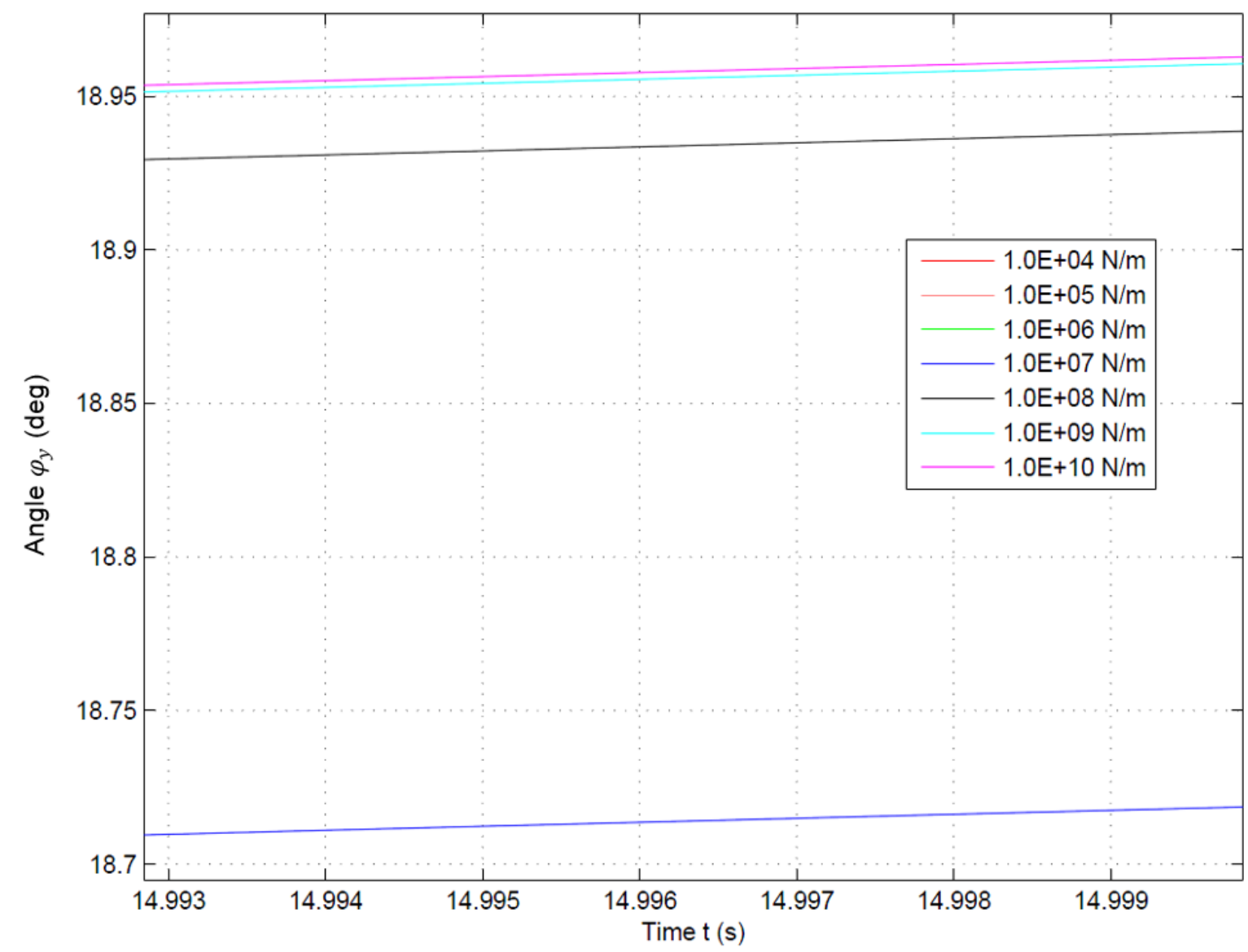

Figure 3.7. Euler Angle (yawing) with different stiffness values (zoom in)

However, not only the Euler Angles are significant parameter to analyze the behavior of system; it is also important to have a closer look at the orbit of the shaft. Thus, the orbit plot of the rotor system with different stiffness settings is plotted in Figure 3.8. The orbit plots describe the displacement in the $\mathrm{x}$ and $\mathrm{y}$-axis of the disk during the rotation.

Due to instability of the system the orbit plot does not reach a circular movement at a stiffness of $1.0 \mathrm{E} 04 \mathrm{~N} / \mathrm{m}$. Also note that with an increasing stiffness the displacement, 
as well as the orbit plot itself becomes smaller. Since there is no significant change in the system with a stiffness above $1.0 \mathrm{E} 07 \mathrm{~N} / \mathrm{m}$ the orbit of the system is not plotted for higher stiffness values.
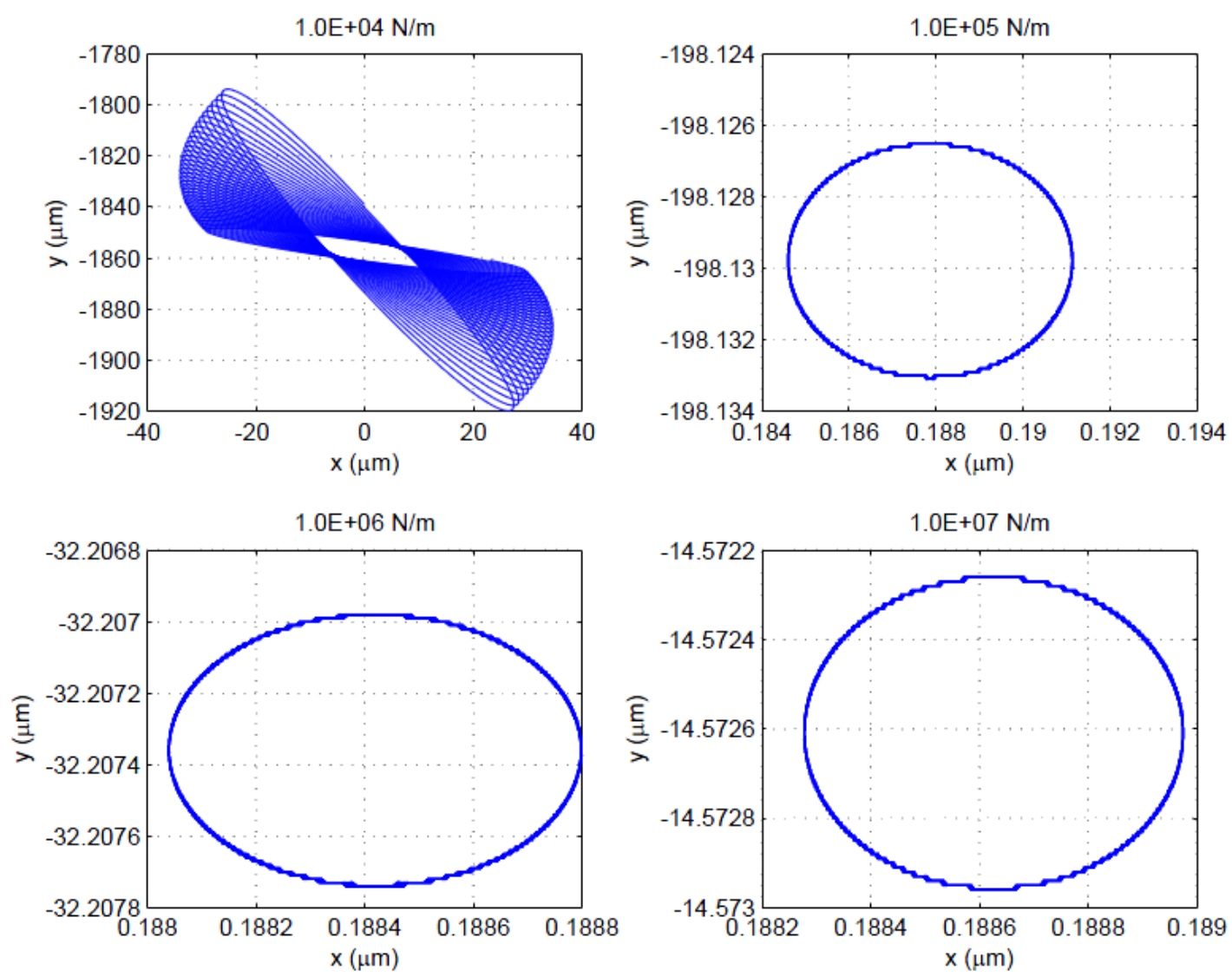

Figure 3.8. Orbit plot of the disk with different stiffness values 


\subsection{Variation of the Disk Location}

\subsubsection{Model Setup}

The next analysis is about the influence on the gyroscopic motion when the location of the disk is changed. For this purpose, several models, each with a different disk location are created. The disk location ratio is used as a reference parameter in this analysis. The disk location ratio is measured by dividing the disk location $a$, which is measured from the left end of the disk, by the total length of the shaft $l$. All other model properties remain the same as in the previous analysis. See Table 3.3 for details.

Table 3.3. Parameter and properties of the model

\begin{tabular}{cccc} 
Symbol & Parameter name & Value & Unit \\
\hline$a$ & Disk location & variable & $\mathrm{m}$ \\
$D$ & Diameter of disk & 0.150 & $\mathrm{~m}$ \\
$d$ & Diameter of shaft & 0.01 & $\mathrm{~m}$ \\
$E$ & Modulus of Elasticity & $2.07 \mathrm{E}+11$ & $\mathrm{~N} / \mathrm{m}^{2}$ \\
$L$ & Disk length & 0.02 & $\mathrm{~m}$ \\
$l$ & Shaft length & 0.5 & $\mathrm{~m}$ \\
$k_{1}$ & Stiffness of Bearing 1 & $1.0 \mathrm{E}+07$ & $\mathrm{~N} / \mathrm{m}$ \\
$k_{2}$ & Stiffness of Bearing 2 & $1.0 \mathrm{E}+07$ & $\mathrm{~N} / \mathrm{m}$ \\
$\rho$ & Density & 7,800 & $\mathrm{~kg} / \mathrm{m}^{3}$ \\
$v$ & Poisson ratio & 0.29 & -
\end{tabular}

In total four different simulation models, each with a different disk location, are created and shown in Table 3.4. Note that only the disk location is a variable parameter, while the length of the shaft remains constant. The output parameter and therefore the measured response is the Euler Angle around the disk. 
Table 3.4. Disk location ratios for each model

Model number

Disk location ratio a/l

\begin{tabular}{ll}
\hline 1 & $1 / 8$ \\
2 & $1 / 4$ \\
3 & $3 / 8$ \\
4 & $1 / 2$ \\
\hline
\end{tabular}

\subsubsection{Results}

The measured response of the GE is the pitching, as well as the yawing angle of the disk, which is presented in Figure 3.9 and Figure 3.10.

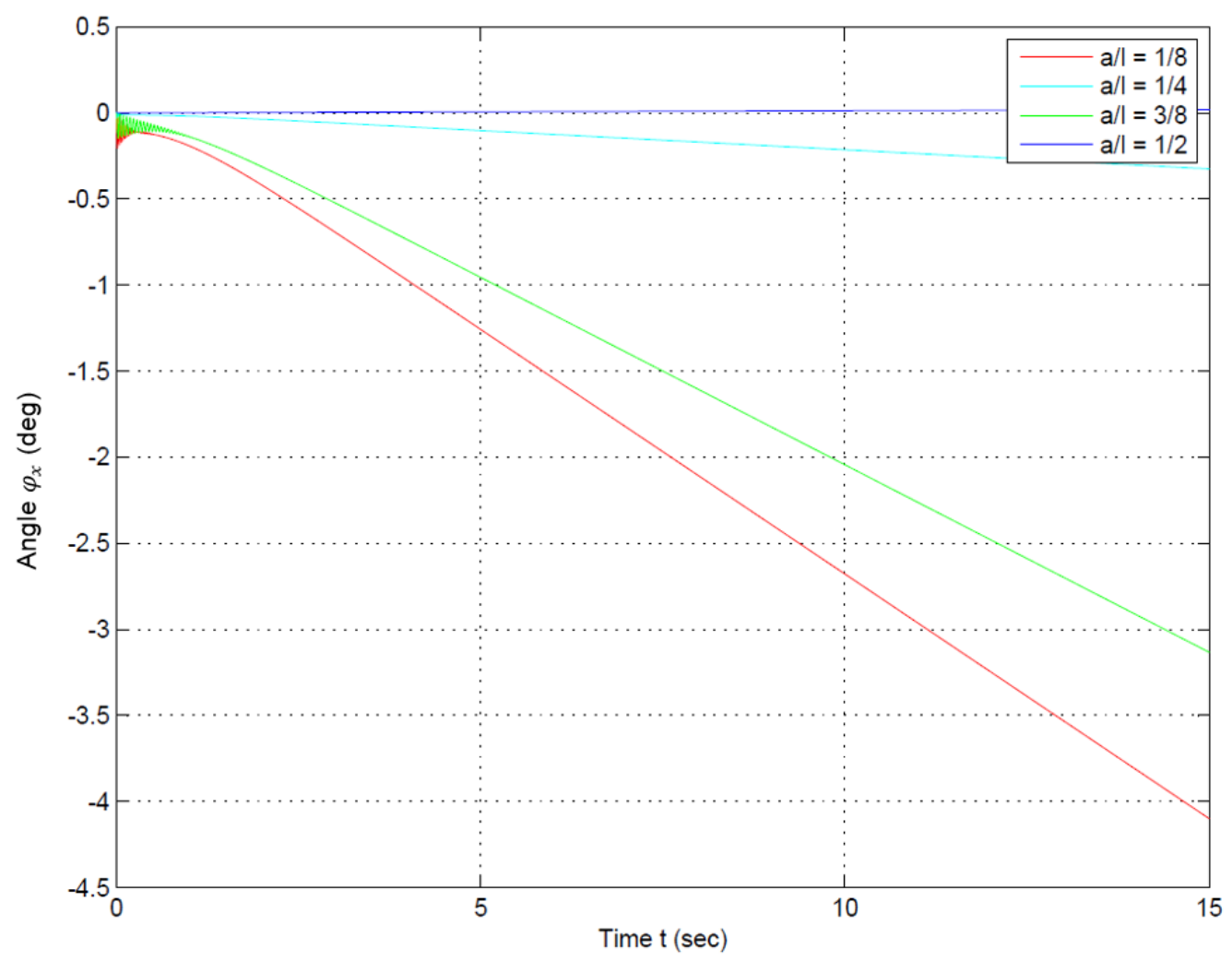

Figure 3.9. Euler Angles (pitching) with different disk locations

Note that the Euler Angle varies with the disk location ratio. For example, the pitching angle shows the highest values at a location of $1 / 8$, if the disk location ratio is increased 
to $1 / 4$ the pitching will be smaller. If the disk location is increased again, the gyroscopic effect will increase again.

Note that if the disk is placed at the center of the shaft (1/2), the pitching, as well as the yawing, will become zero. In other words, the gyroscopic effect will disappear, when the disk is placed at the center of the shaft. This is an important observation, since the rotor system reduces to a so called Jeffcott-Rotor system then. The Jeffcott-Rotor does not include any gyroscopic effects, since the inertia of the system does not contribute to the gyroscopic effect.

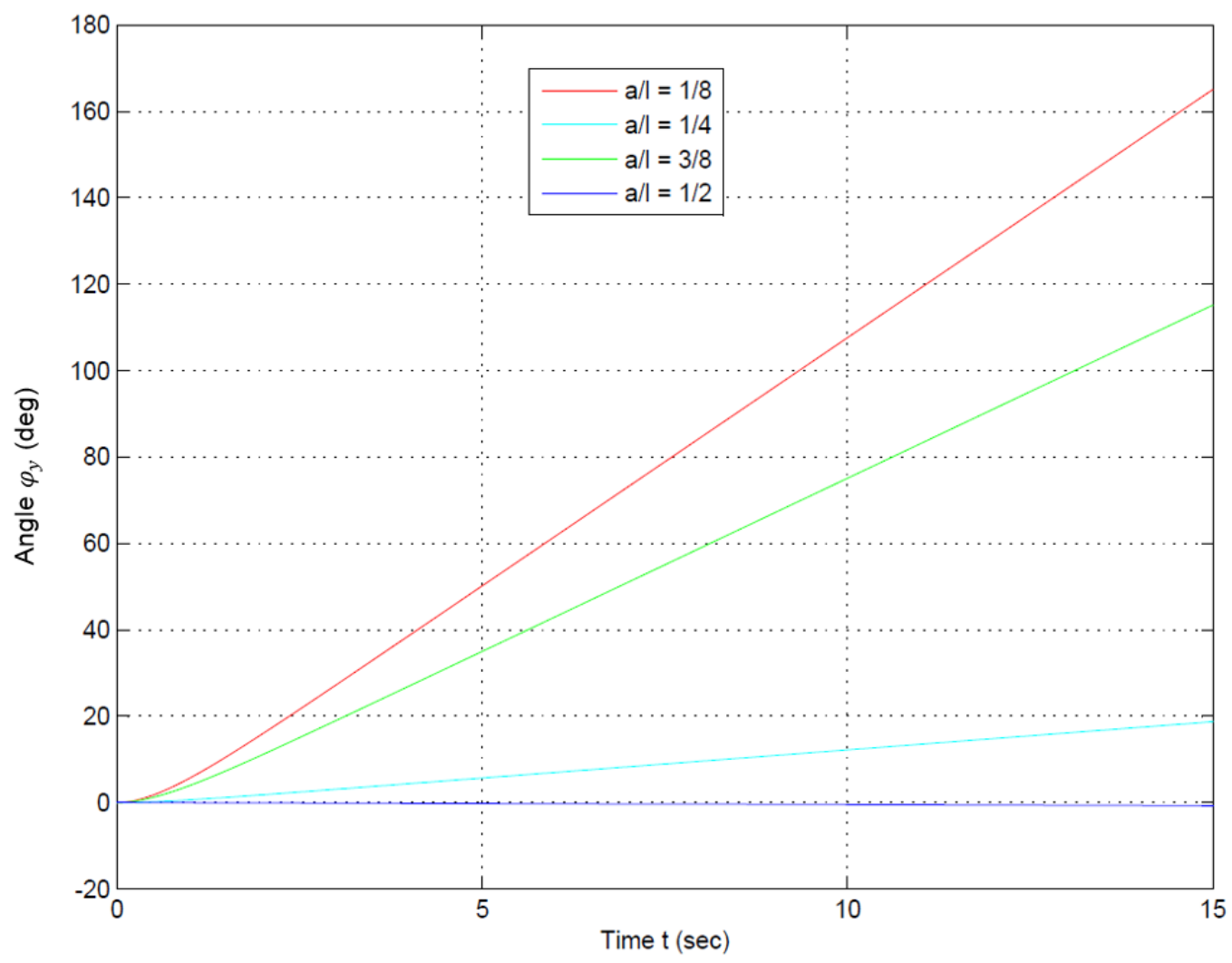

Figure 3.10. Euler angles (yawing) with different disk locations

Similarities exist if the yawing motion of the disk is analyzed. The yawing increases with the off-centered rotor disk, except for a value of $1 / 4$. Furthermore, the yawing reduces to zero if the disk location ratio is $1 / 2$, which confirms the observation that no 
gyroscopic motion is present when a Jeffcott-rotor is used. It is also worthwhile to observe that the yawing motion is in general greater than the pitching motion and is present in the opposite direction.

Furthermore, the orbit plot of the shaft-disk system is shown in Figure 3.11. As you can see the smallest orbit plot exist at a disk location ratio of $1 / 4$.
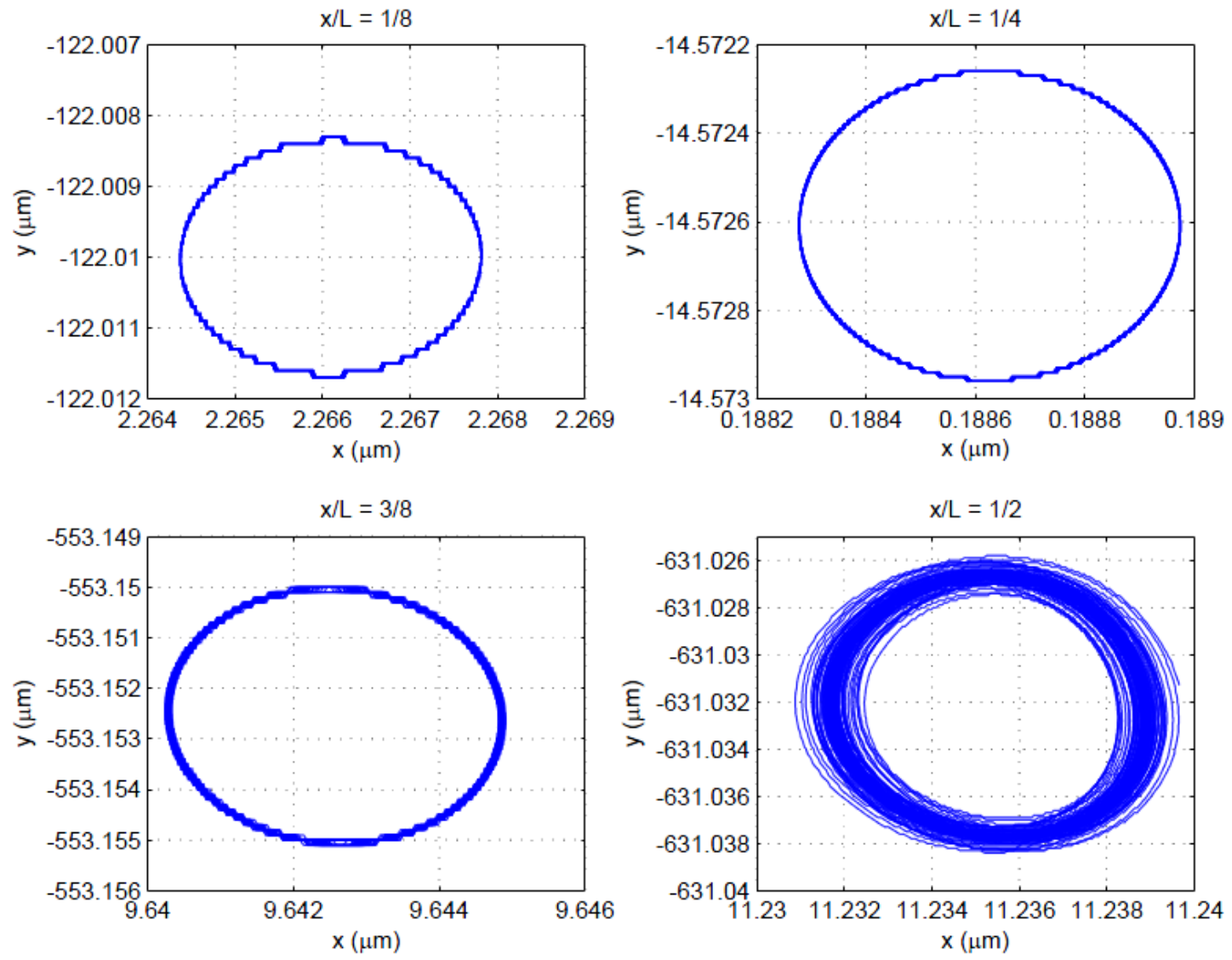

Figure 3.11. Orbit plots with different disk positions 


\subsection{Influence of Eccentricities}

The objective of this section is to observe the influence of the shaft-disk system if the disk is unbalanced. Therefore, several different masses will be placed on the disk in order to create an unbalanced rotor. Figure 3.12 shows the single disk system in Adams. The disk of the rotor system is located at $1 / 4$ of the shafts length. All other properties, as well as dimension of the disk, remain the same as in the previous models. In order to create an unbalanced rotor, a mass is added to the disk, which is placed at the outer diameter of the disk. The mass is created using a spherical body in Adams with the properties of steel. The radius of the sphere is varied in order to create different weights, which are shown in Table 3.5. The motion is constant and has a value of 15 $\mathrm{Hz}$.

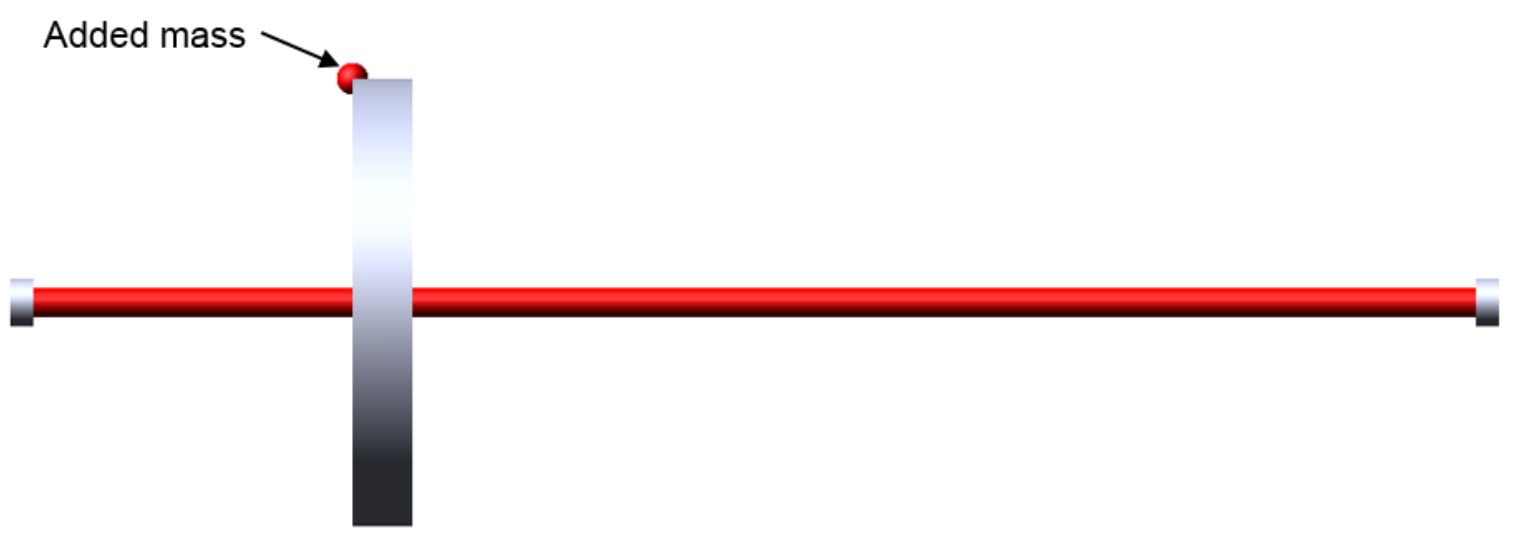

Figure 3.12. Unbalanced shaft-disk system in Adams

Table 3.5. Radius and mass of the eccentricities Radius of sphere Mass

$5.35 \mathrm{~mm}$ $5 \mathrm{~g}$

$6.74 \mathrm{~mm}$

$10 \mathrm{~g}$

$8.49 \mathrm{~mm}$

$20 \mathrm{~g}$

$10.7 \mathrm{~mm}$

$40 \mathrm{~g}$ 
Figure 3.13 shows the result in form of the orbit plot. Note that once the weight of the eccentricity increases, the orbit plot will increase too. This is reasonable since the unbalanced system will create a higher excitation of the rotor, resulting in an increase of the displacement.
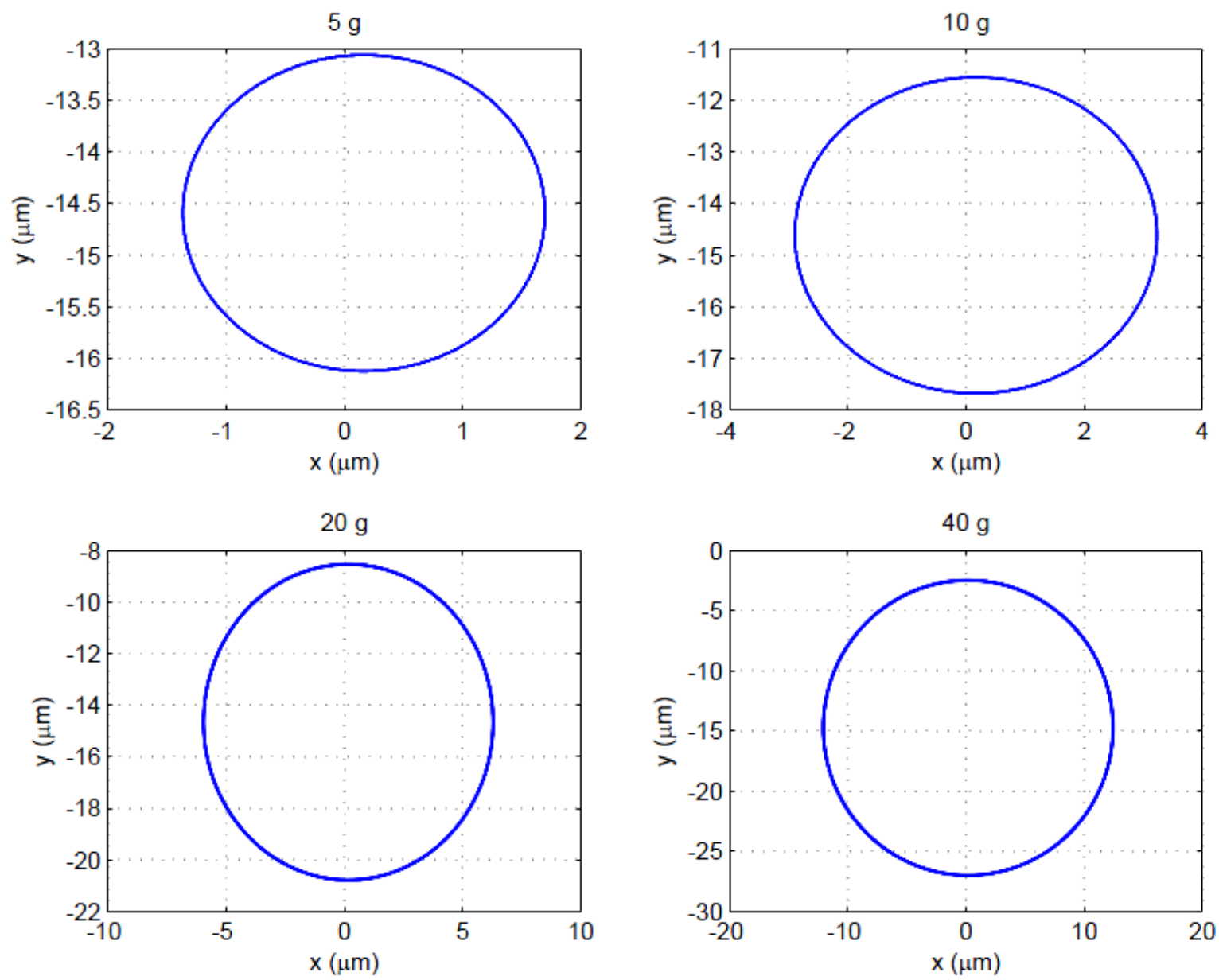

Figure 3.13. Orbit plots at $15 \mathrm{~Hz}$ with different eccentricities

To demonstrate that there is an effect on the vibrations, the FFT plot with different masses are shown in Figure 3.14. As you can see, with a mass of $0 \mathrm{~g}$ there is almost no peak in the vibration spectrum. Once the mass increases, the amplitude of the vibrational response becomes proportional larger. Therefore, it is very important to pay attention to the eccentricity and try to unbalance a rotor in order to reduce the vibration response. 


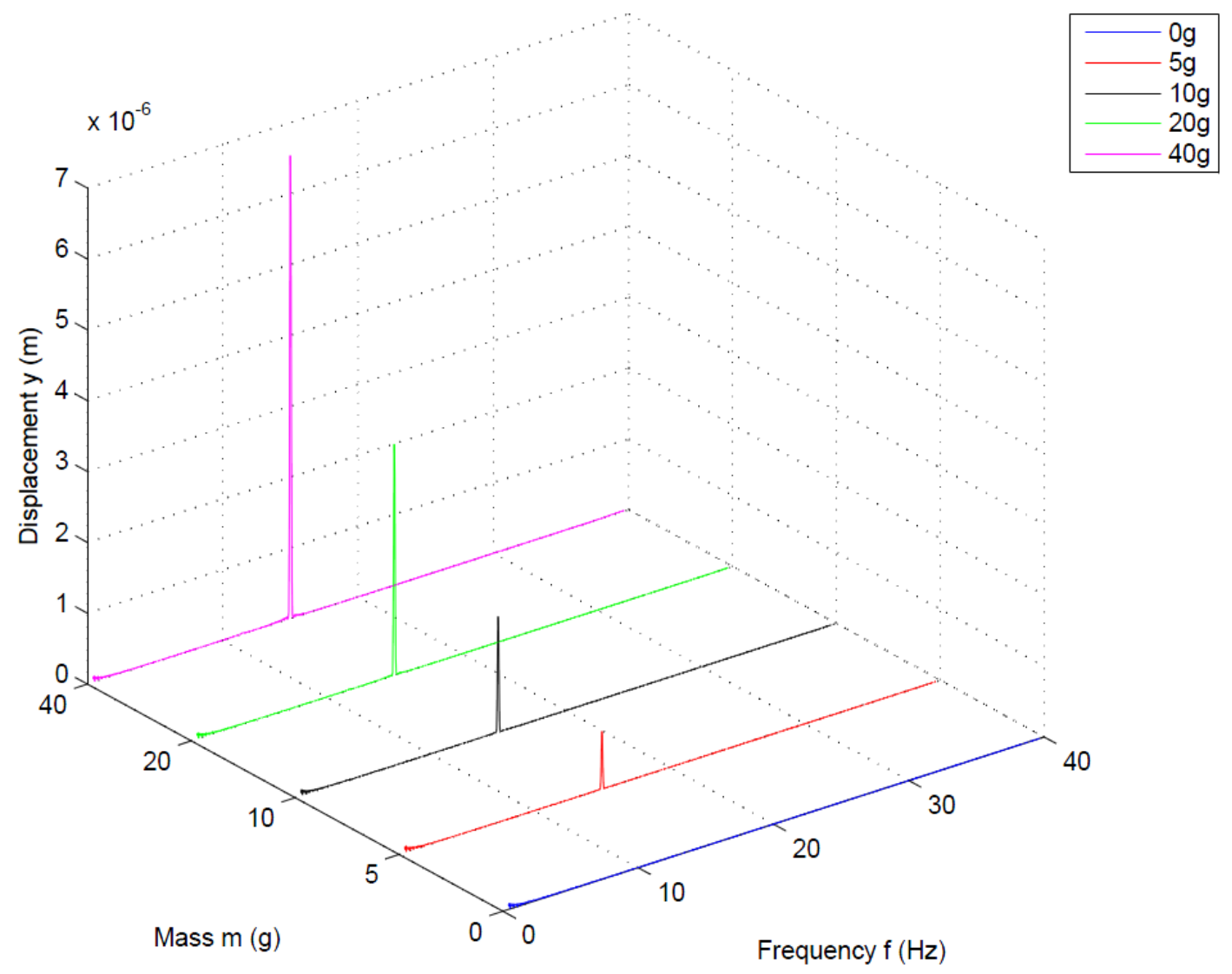

Figure 3.14. Frequency response at $15 \mathrm{~Hz}$ with different eccentricities

\subsection{Analysis of an Anisotropic Bearing System}

Furthermore, it is significant to analyze the shaft-disk system if anisotropic bearings are used. Anisotropic bearings are bearings that have different properties in each direction. To create an anisotropic bearing system in Adams, the bushing stiffness will be changed. For instance, in this example the bearing stiffness in the (vertical) y direction remains constant at $1.0 \mathrm{E} 7 \mathrm{~N} / \mathrm{m}$, while the stiffness in the horizontal direction ( $x$-direction) is reduced to $1.0 \mathrm{E} 06 \mathrm{~N} / \mathrm{m}$. The orbit plot of this analysis is presented in Figure 3.15. Note the orbit of the anisotropic bearing does not show an elliptical shape. This is due to the reduced stiffness in the $x$ direction. Note also, that the orbit increases if anisotropic bearings are used. 

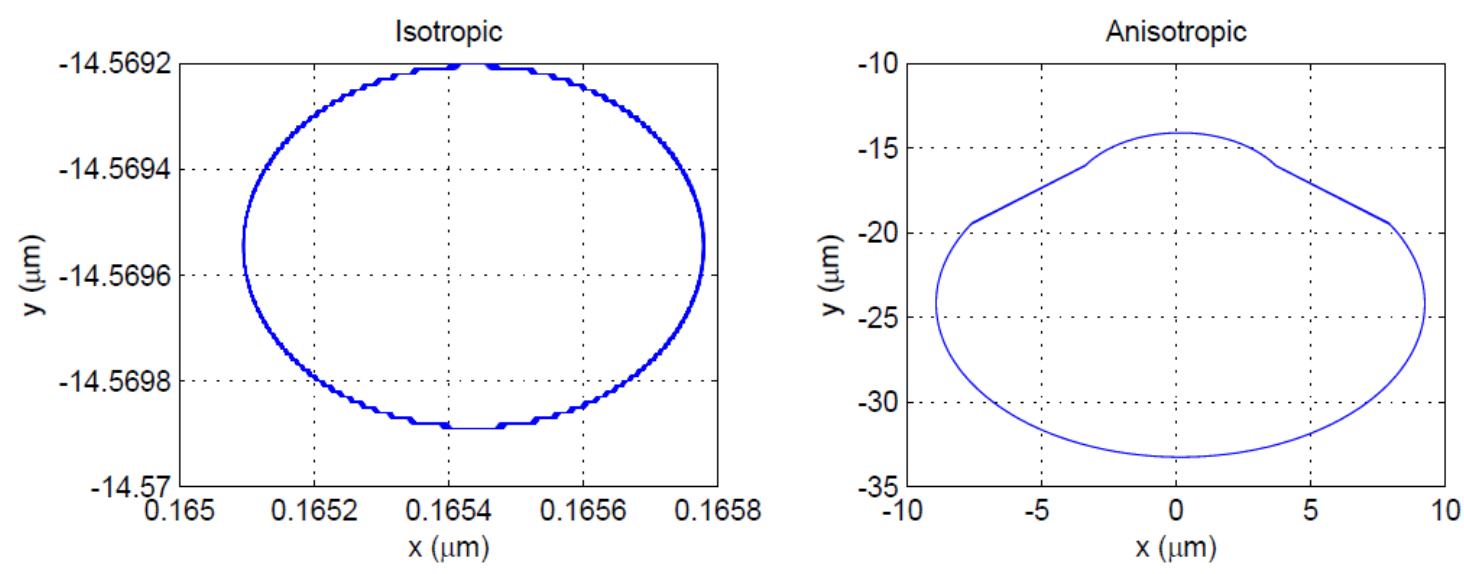

Figure 3.15. Orbit plot with anisotropic bearings 


\section{Health Monitoring with a Simple Pair of Gears}

\subsection{General Model Setup}

This simulation consist of a simple pair of gears, mounted on flexible shafts that represents a fixed axis gear system. Each shaft is supported by bearings, which are modeled using the bushing option and ensures that the setup is modeled as realistic as possible. Figure 4.1 shows the general model setup in Adams. Furthermore, a rotational speed, as well as a torque, is applied to the pinion and a resistant torque is applied to the output shaft. The speed profile, as well as the torque profile, will be explained in more detail in this chapter.

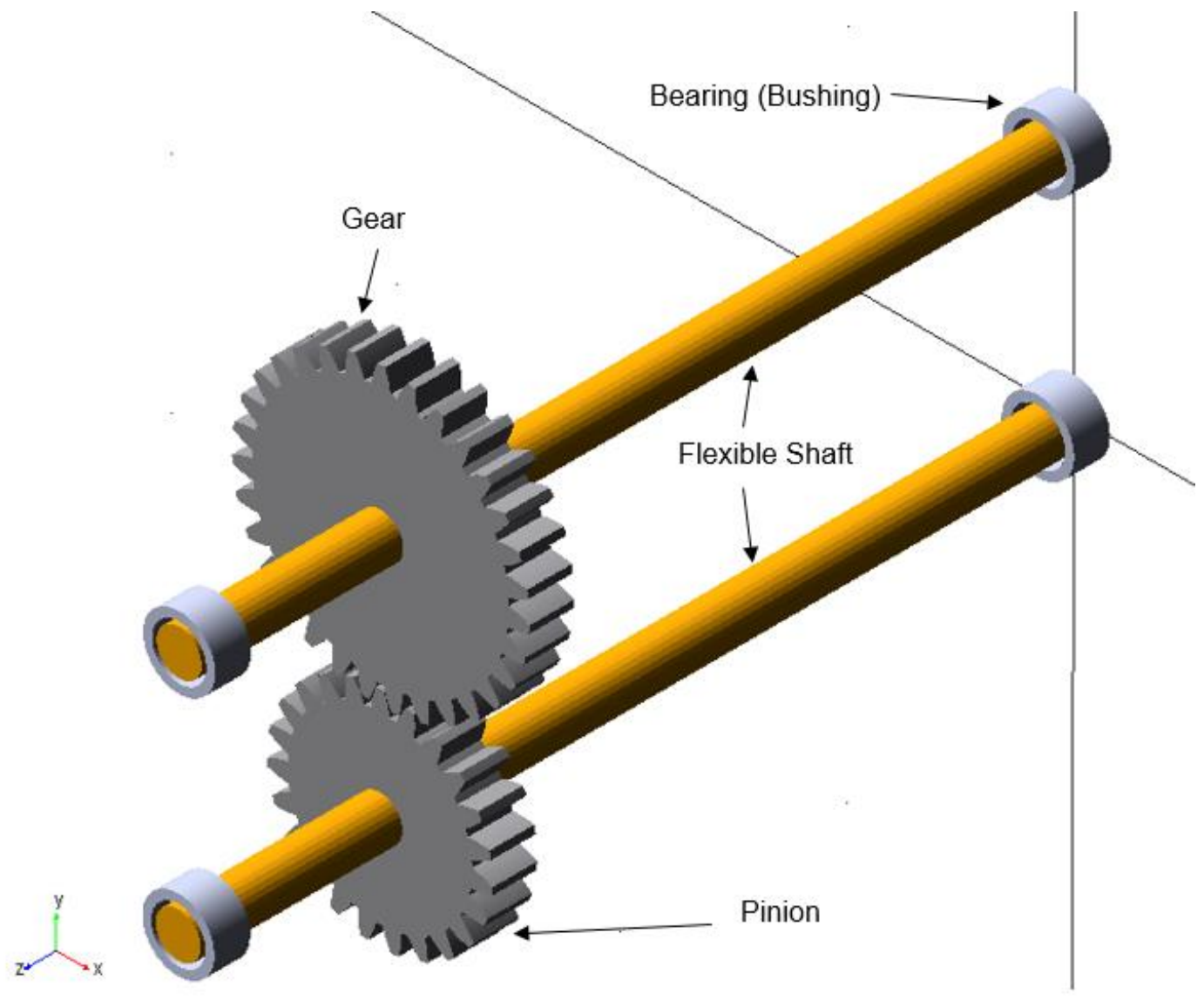

Figure 4.1. Simple pair of gears system in Adams

Figure 4.1 shows the general properties of the gearing system. The properties of the shaft are shown in Table 4.1 and the properties of the flexible shaft are presented in 
Table 4.2. Again, the flexible bodies are created in Abaqus and imported in Adams. Although the gearing system is small, it can be used to represent a real system and scaled if necessary for further studies.

Table 4.1. Properties of the gears

\begin{tabular}{c|cc}
\multicolumn{2}{c}{ PINION } & GEAR \\
\hline NUMBER OF THEETH & 23 & 31 \\
MODULE & $2 \mathrm{~mm}$ & $2 \mathrm{~mm}$ \\
PRESSURE ANGLE & $20^{\circ}$ & $20^{\circ}$ \\
BORE DIAMETER & $10 \mathrm{~mm}$ & $10 \mathrm{~mm}$ \\
WIDTH & $10 \mathrm{~mm}$ & $10 \mathrm{~mm}$ \\
MATERIAL & steel $^{2}$ & steel \\
\hline
\end{tabular}

Table 4.2. Properties of the shaft

\begin{tabular}{cccc} 
Symbol & Parameter & Value & Unit \\
\hline$d$ & Diameter of shaft & 0.01 & $\mathrm{~m}$ \\
$E$ & Modulus of Elasticity & $2.07 \mathrm{E}+11$ & $\mathrm{~N} / \mathrm{m}^{2}$ \\
$l$ & Shaft length & 0.2 & $\mathrm{~m}$ \\
$k$ & Bearingstiffness & $1.0 \mathrm{E}+07$ & $\mathrm{~N} / \mathrm{m}$ \\
$\rho$ & Density & 7,800 & $\mathrm{~kg} / \mathrm{m}^{3}$ \\
$v$ & Poisson ratio & 0.29 & - \\
\hline
\end{tabular}

2 This default material definition for rigid bodies is chosen in Adams. 


\subsection{Modeling the Speed and Torque Profile}

Realistic simulation models can only be achieved if the inputs are as realistic as possible. To ensure a practical gear model, the input torque will represent an AC-motor curve, which is dependent on the input speed. However, the values of the curve are chosen arbitrary, but the shape of the function resembles a practical motor curve. The torque profile, which is specified in Adams is shown in Figure 4.2.

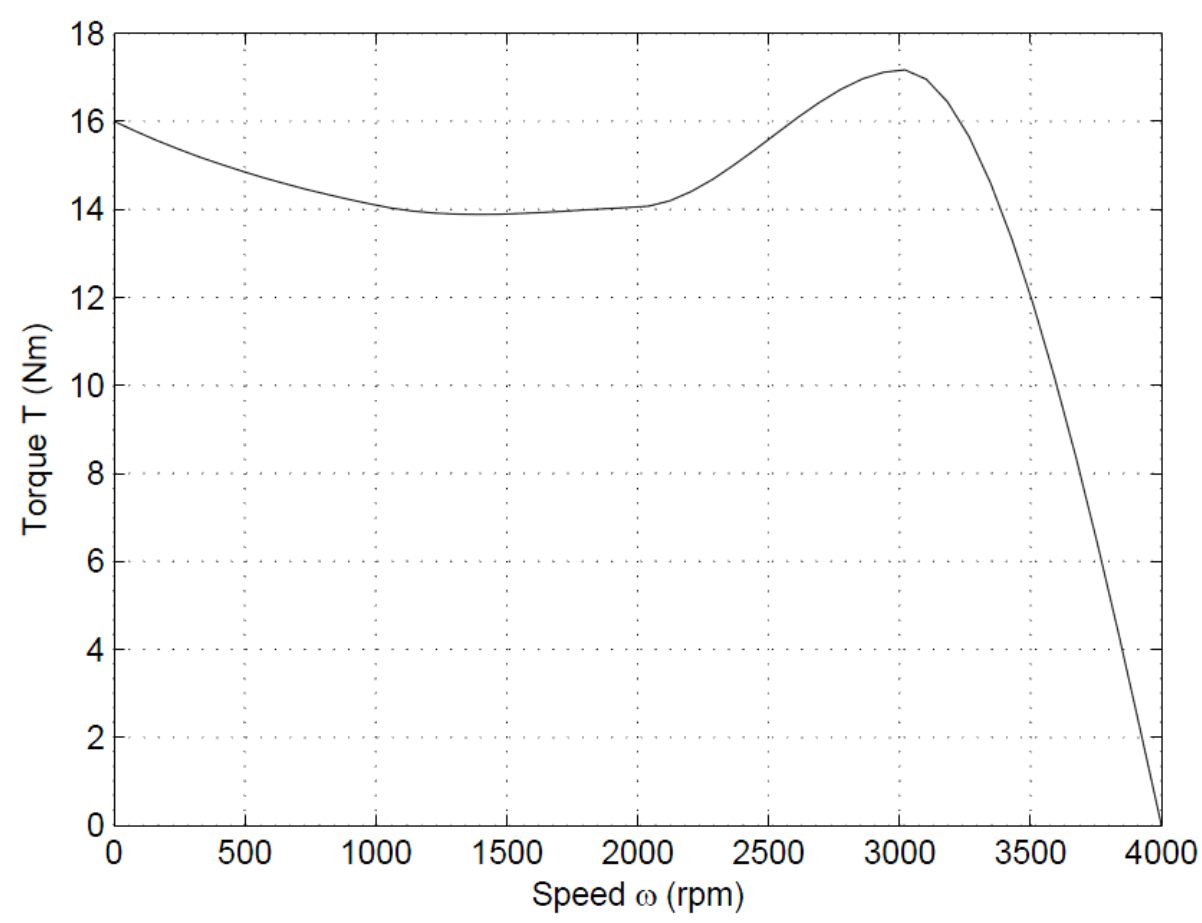

Figure 4.2. Input torque profile

It is also reasonable to define different speed profiles. In a real system, any imaginable speed profile could be possible and present. For this purpose, three different speed profiles are defined and used for the dynamic analysis, shown in Figure 4.3.

The first speed profile represents a constant speed, at which the shaft is rotating constantly at $1200 \mathrm{rpm}$ during the whole simulation time. In addition, an exponential speed curve is modeled where the speed value of $1200 \mathrm{rpm}$ is reached without overshooting. Lastly, a liner increasing speed profile is applied to the system in which the whole range of the torque curve is exploited $(0-4000 \mathrm{rpm})$. 


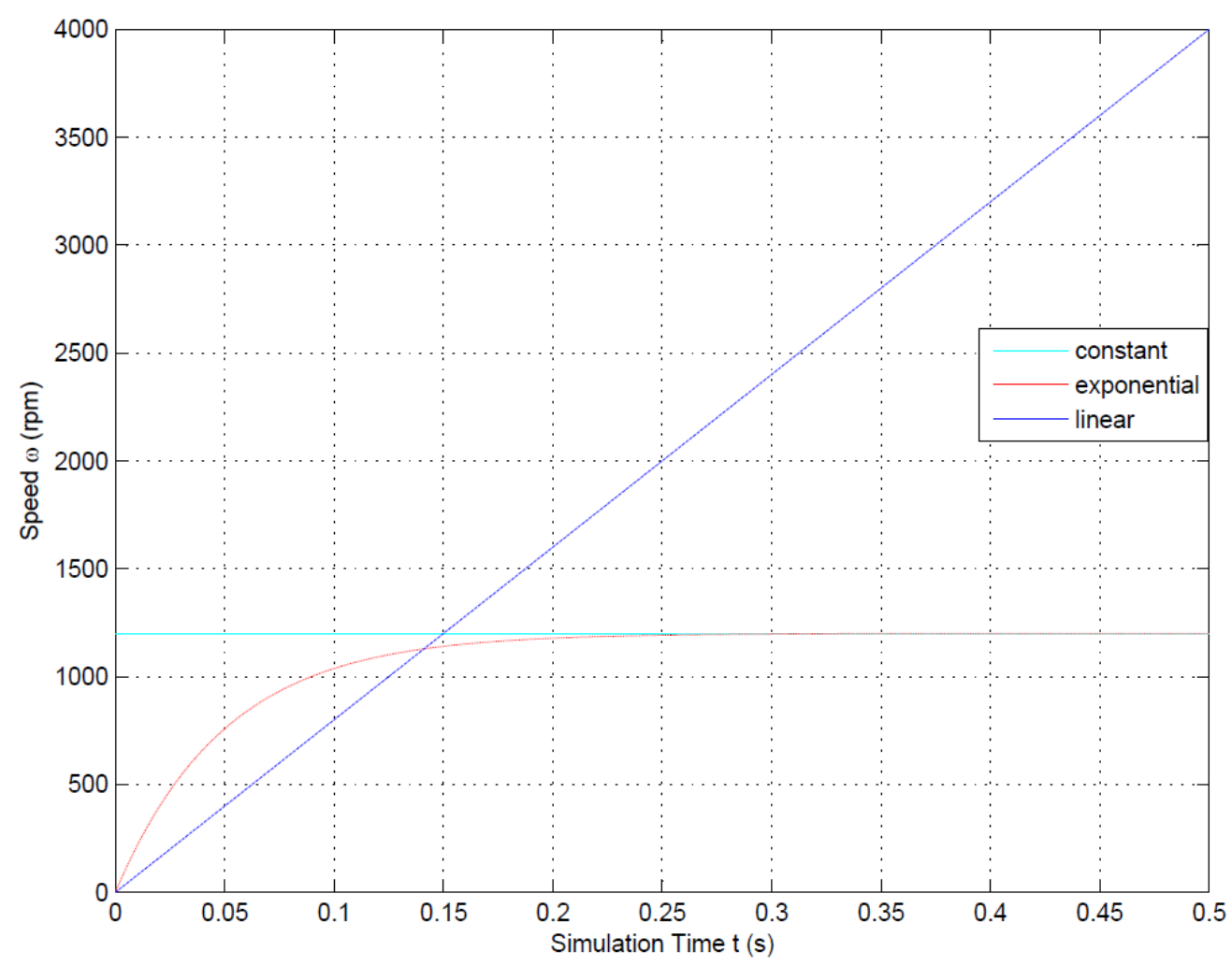

Figure 4.3. Input speed profiles

This input setup will be used to perform the simulation experiments. Although the defined speed profiles and the torque curve are only a tiny fraction of different possible practical setups, they are important for this study in order to design a realistic Adams model.

As mentioned before, a resisted torque is also present in the system in order to ensure that a resistance exists in this system. The resistance torque is a function of the input and output torque, as well as the input and output speeds. Furthermore, an efficiency factor for the gearing system $\eta_{G}$ of 0.98 is included. A realistic range of the gear efficiency varies between $94-98 \%$ [18].

$$
T_{\text {res }}=\frac{T_{\text {in }} \omega_{\text {in }}}{\omega_{\text {out }}} \eta_{G}
$$




\subsection{Faults and Defects in the Gear System}

To ensure proper health monitoring and fault detection, several defects are included in the gear system. The first defect is a damaged tooth that is located at the pinion. The chipped tooth can be the result of material faults or caused by fatigue. The chipped tooth is presented in Figure 4.4.

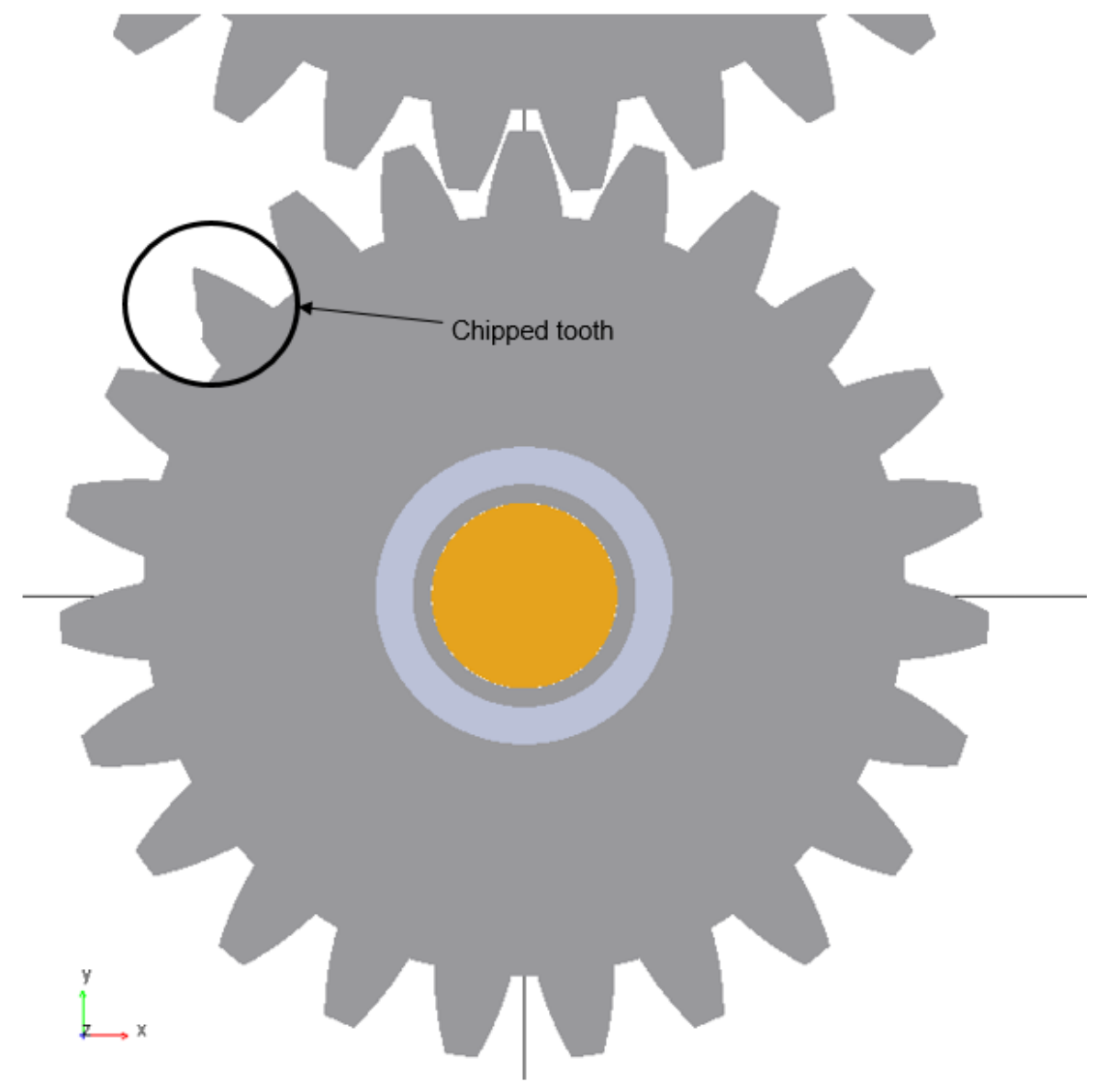

Figure 4.4. Chipped pinion

The next gear defect includes a cracked tooth located at the driving gear (pinion). In order to create this tooth defect, the gear must be created as a flexible body, which is realized with Abaqus, too. The same material properties that are defined for the flexible shafts applies for the gear. The crack is $0.01 \mathrm{~mm}$ thick and has a length of about $50 \%$ of the tooth width, shown in Figure 4.5. 


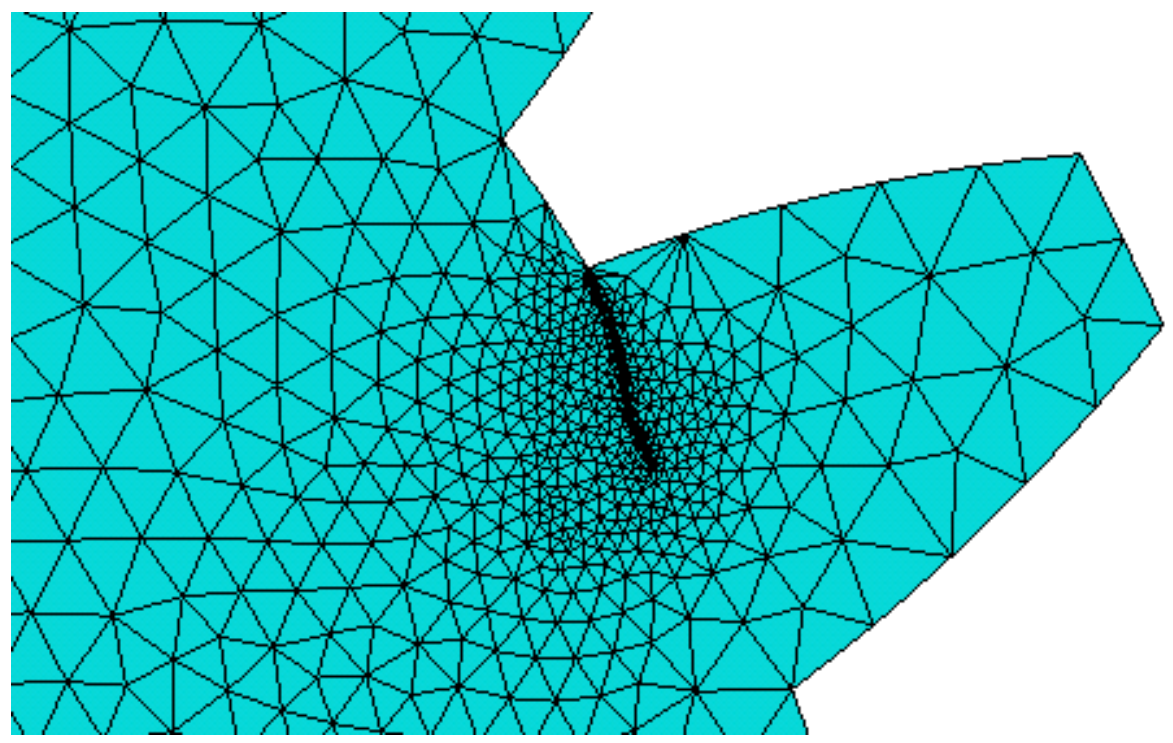

Figure 4.5. Cracked pinion

Not only a crack in the gear can be present in a gear box, it is also imaginable that a crack in the shaft can exist. Therefore, another fault will be included in the system, which is a cracked shaft. The crack in the shaft is $0.2 \mathrm{~mm}$ thick and has a depth of 5 $\mathrm{mm}$, which is the half of the shafts' cross section. It occurs at the center (half length) of the shaft and is placed at the driving shaft. The crack in the shaft is shown in Figure 4.6.

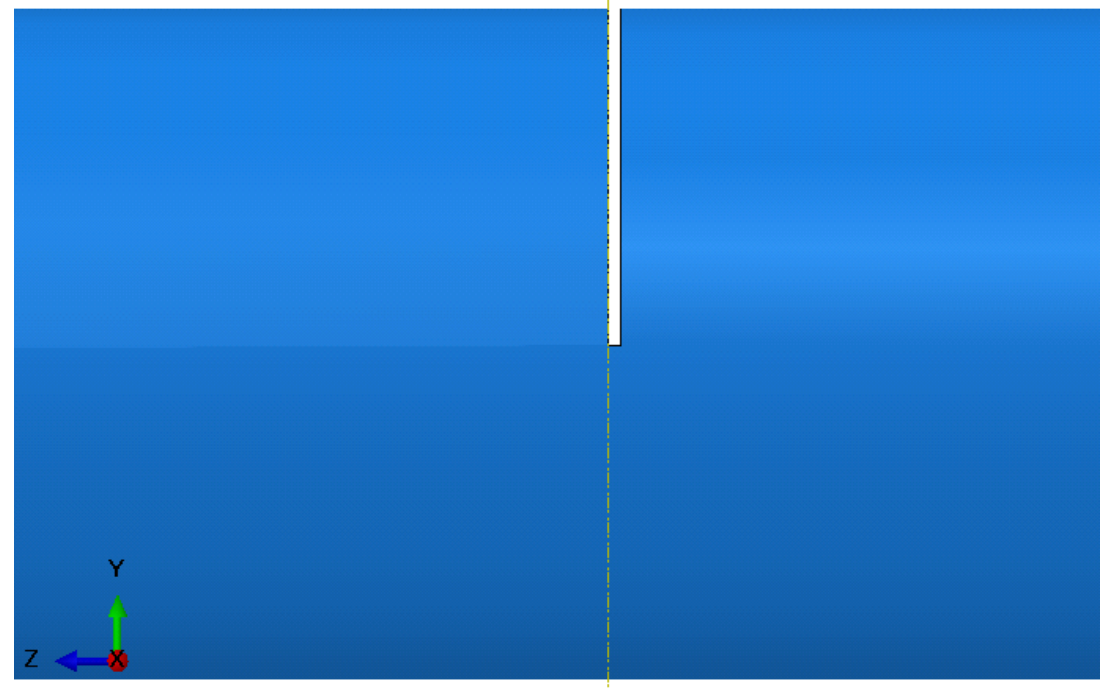

Figure 4.6. Cracked shaft 


\subsection{Simulation Procedure and Analysis Scope}

An effective analysis presupposes a well-planned experimental method, as well as well-organized simulation procedure. This includes the definition of the inputs and outputs, as well as the analysis scope, which are described below.

The inputs of the system are:

- Torque, defined by the torque-speed curve

- Speed profile:

- Constant speed $(10 \mathrm{~Hz}, 20 \mathrm{~Hz}, 30 \mathrm{~Hz}, 40 \mathrm{~Hz}, 50 \mathrm{~Hz}, 60 \mathrm{~Hz})$

- Exponential

- Linearly increasing

While the output of the system is:

- Contact force between the two gears

In addition, the simulation setups are:

- Healthy/Perfect Gear

- Chipped tooth

- Cracked tooth

- Cracked Shaft

The analysis is performed by:

- FFT

- 3D FFT

- Wavelet

- Statistical methods 


\subsection{Analysis of the Flexibility of the Shaft and the Bearings}

Before the actual simulation is completed, an analysis of the different possible flexibility modes in Adams is carried out. Therefore, a healthy simple pair of gear system is modeled with different flexibilities of the shaft and bearings. These different possible types of flexibilities can contain a rigid-shaft and rigid-bearing (RSRB)-system, a rigidshaft and flexible-bearings system (RSFB) and a flexible-shaft and flexible-bearings (FSFB) system. A simulation in Adams is performed with each model at a constant speed of $1200 \mathrm{rpm}(20 \mathrm{~Hz})$. The contact force between the two gears is measured and FFT is used to display the gear mesh frequencies (GMF) of the system. The result is shown in Figure 4.7 and the values are presented in Table 4.3.

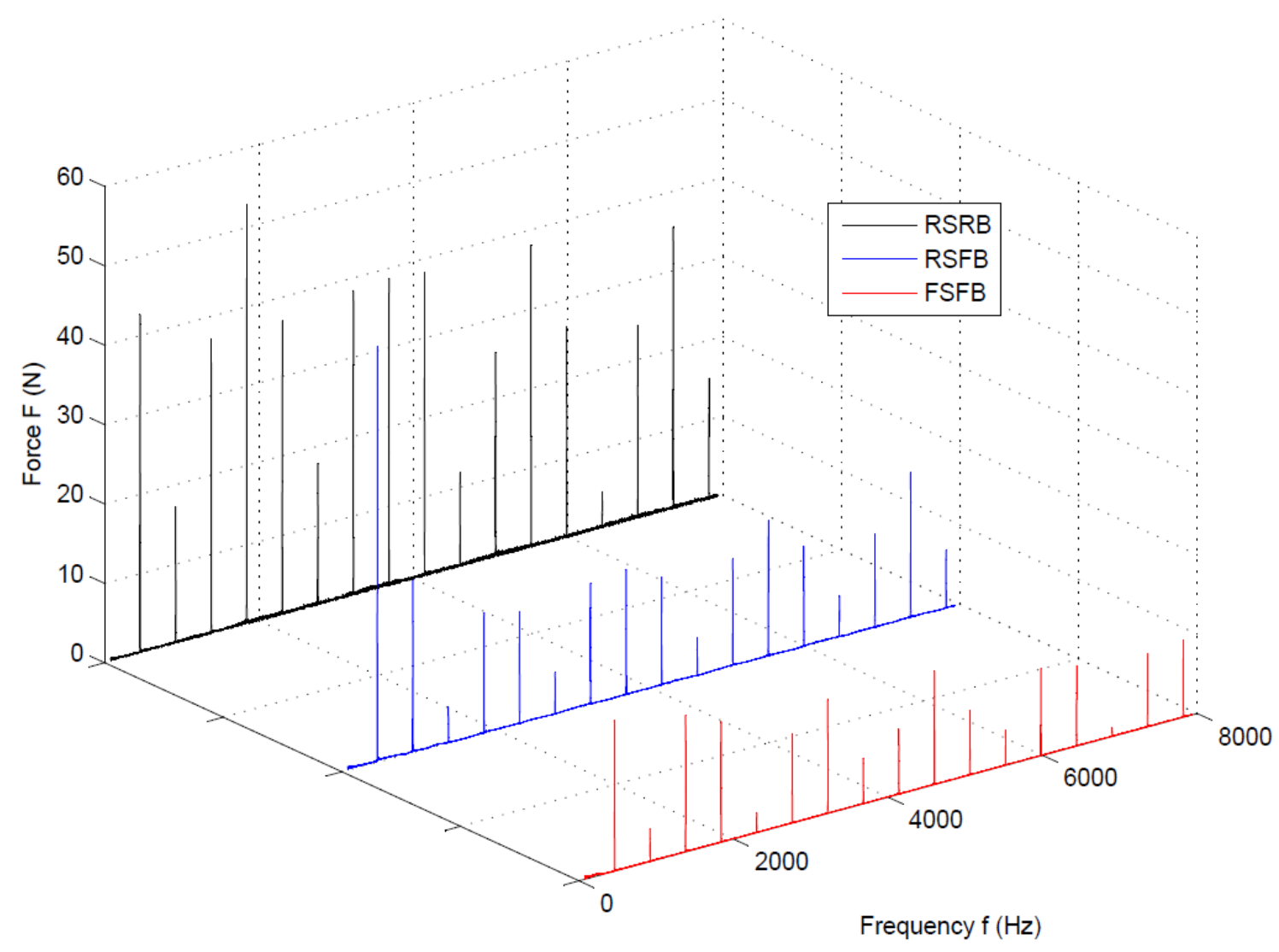

Figure 4.7. GMF comparison with different flexibilities 
Note, once the model becomes more flexible, the magnitude of the harmonics decreases. Thus, a system with a flexible body and flexible bearings shows the lowest value of almost all GMF-peaks. The reason for that is that the flexibility of the model enables to absorb the force. A flexible FEA body acts as a spring, stores more energy and therefore shows a lower magnitude in the FFT-spectrum. On the other hand, a rigid body does not have the capability of storing the energy and reveals higher magnitudes for the GMFs.

Table 4.3. Numerical values of the GMFs with different flexibilities

\begin{tabular}{c|ccc} 
GMF & RSRB & RSFB & FSFB \\
\hline $460 \mathrm{~Hz}$ & $42.65 \mathrm{~N}$ & $52.36 \mathrm{~N}$ & $19.00 \mathrm{~N}$ \\
$920 \mathrm{~Hz}$ & $17.28 \mathrm{~N}$ & $22.08 \mathrm{~N}$ & $4.19 \mathrm{~N}$ \\
$1380 \mathrm{~Hz}$ & $37.13 \mathrm{~N}$ & $4.52 \mathrm{~N}$ & $17.24 \mathrm{~N}$ \\
$1840 \mathrm{~Hz}$ & $51.92 \mathrm{~N}$ & $15.24 \mathrm{~N}$ & $15.16 \mathrm{~N}$ \\
$2300 \mathrm{~Hz}$ & $37.02 \mathrm{~N}$ & $14.16 \mathrm{~N}$ & $2.47 \mathrm{~N}$ \\
$2760 \mathrm{~Hz}$ & $17.87 \mathrm{~N}$ & $5.23 \mathrm{~N}$ & $11.23 \mathrm{~N}$ \\
$3220 \mathrm{~Hz}$ & $38.32 \mathrm{~N}$ & $15.28 \mathrm{~N}$ & $14.31 \mathrm{~N}$ \\
$3680 \mathrm{~Hz}$ & $38.76 \mathrm{~N}$ & $15.80 \mathrm{~N}$ & $5.75 \mathrm{~N}$ \\
$4140 \mathrm{~Hz}$ & $38.20 \mathrm{~N}$ & $13.64 \mathrm{~N}$ & $8.02 \mathrm{~N}$ \\
$4600 \mathrm{~Hz}$ & $11.81 \mathrm{~N}$ & $4.78 \mathrm{~N}$ & $14.28 \mathrm{~N}$ \\
$5060 \mathrm{~Hz}$ & $25.81 \mathrm{~N}$ & $13.47 \mathrm{~N}$ & $8.18 \mathrm{~N}$ \\
$5520 \mathrm{~Hz}$ & $38.07 \mathrm{~N}$ & $17.16 \mathrm{~N}$ & $4.37 \mathrm{~N}$ \\
$5980 \mathrm{~Hz}$ & $26.56 \mathrm{~N}$ & $12.68 \mathrm{~N}$ & $10.95 \mathrm{~N}$ \\
$6440 \mathrm{~Hz}$ & $4.46 \mathrm{~N}$ & $5.21 \mathrm{~N}$ & $10.17 \mathrm{~N}$ \\
$6900 \mathrm{~Hz}$ & $24.36 \mathrm{~N}$ & $11.80 \mathrm{~N}$ & $1.08 \mathrm{~N}$ \\
$7360 \mathrm{~Hz}$ & $35.46 \mathrm{~N}$ & $18.26 \mathrm{~N}$ & $9.22 \mathrm{~N}$ \\
$7820 \mathrm{~Hz}$ & $15.27 \mathrm{~N}$ & $7.30 \mathrm{~N}$ & $9.71 \mathrm{~N}$ \\
\hline
\end{tabular}




\subsection{Health Monitoring with FFT and 3D FFT}

\subsubsection{FFT and Time Domain Analysis}

The first study includes the analysis of the frequency domain and the time domain of the contact force between the gears. Therefore, the frequency spectrum and the time domain of the contact force will be plotted. The FFT signal includes the gear mesh frequencies, which appear as peaks in the FFT plot. The individual faults such as the chipped pinion, the cracked pinion and the cracked pinion will be compared to a perfect gear set.

\subsubsection{Chipped Pinion}

The signal of the contact force of a healthy and a chipped pinion in the time domain is shown in Figure 3.8. As you can see, even the time domain signal allows the possibility of condition monitoring. While the response of the healthy pinion shows a constantly oscillating signal, the chipped pinion shows additional high contact force peaks in every interval of $0.05 \mathrm{~s}(20 \mathrm{~Hz})$ that reaches values up to $2200 \mathrm{~N}$. Since this system creates every rotation high impact forces at a single location, it can be revealed that a fault in the gearing system is present. Even the location of the fault can be located if the starting position of the gearing system is known.

However, it is not always guaranteed that a fault can be detected from the time domain signal. Moreover, the meshing forces that create high peaks in the system might be an exception and could also be much lower and therefore not always be visible. For this purpose, it is more suitable to analyze the frequency spectrum of the signal, since it reveals more information of a signal. The frequency spectrum of the above signal is shown in Figure 4.9. 

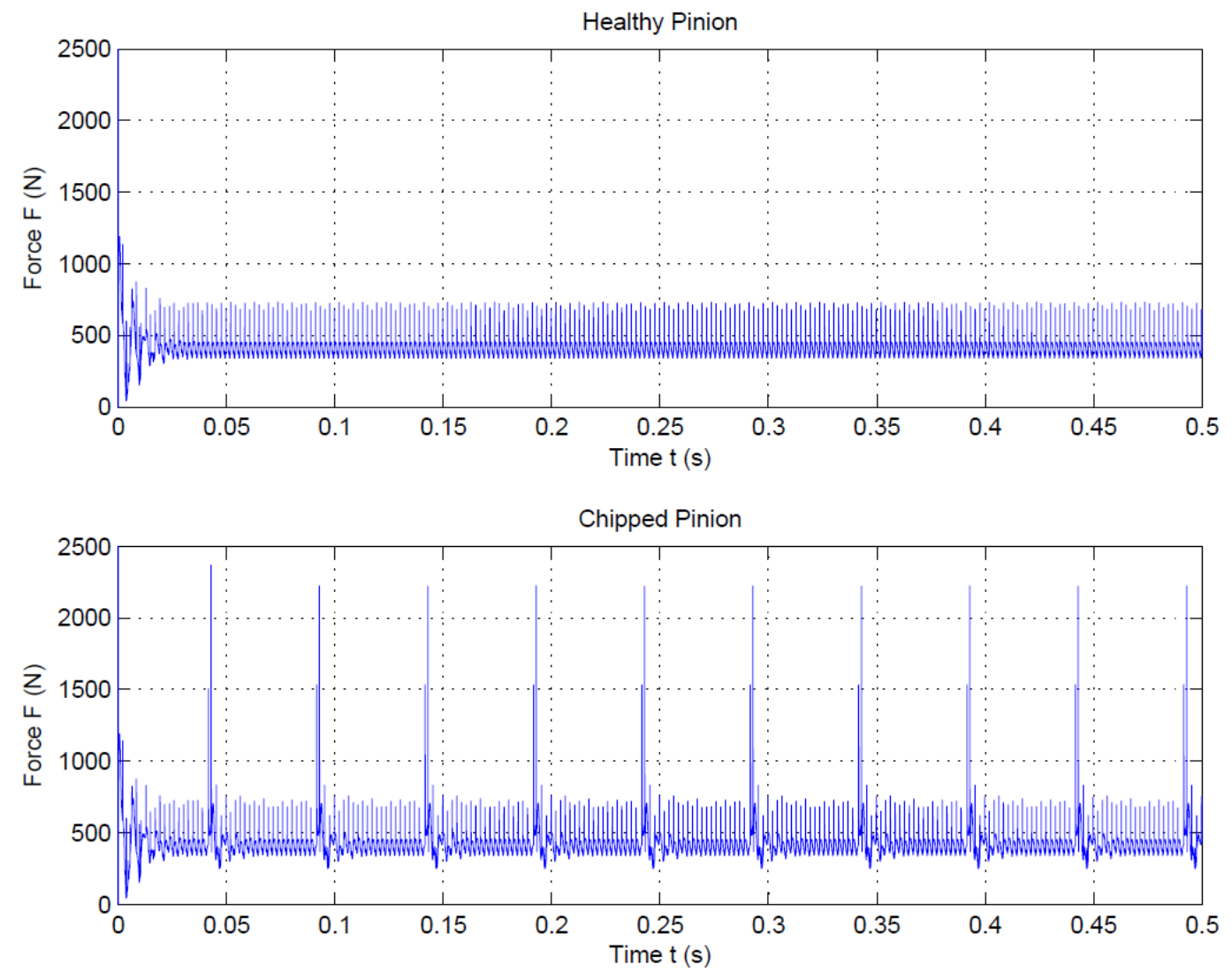

Figure 4.8. Contact force in the time domain of the healthy and chipped pinion

The peaks in the vibration spectrum represent the gear mesh frequencies and are created during the meshing process of the teeth. While the FFT of the healthy signal has a neat and clear form, the FFT of the chipped pinion contains a heavy noise profile. The noise of the vibration is due to the defect in the pinion and is present in form of sidebands on each side of each gear mesh frequency peaks. Note that for the second gear mesh frequency the sideband are even higher that the magnitude of the peak. This information can be used to create an automated health monitoring system. One method is the Side Band Energy Ratio, which will be explained in more detail later in this chapter, and which allows the possibility of fault detection using an algorithm. 

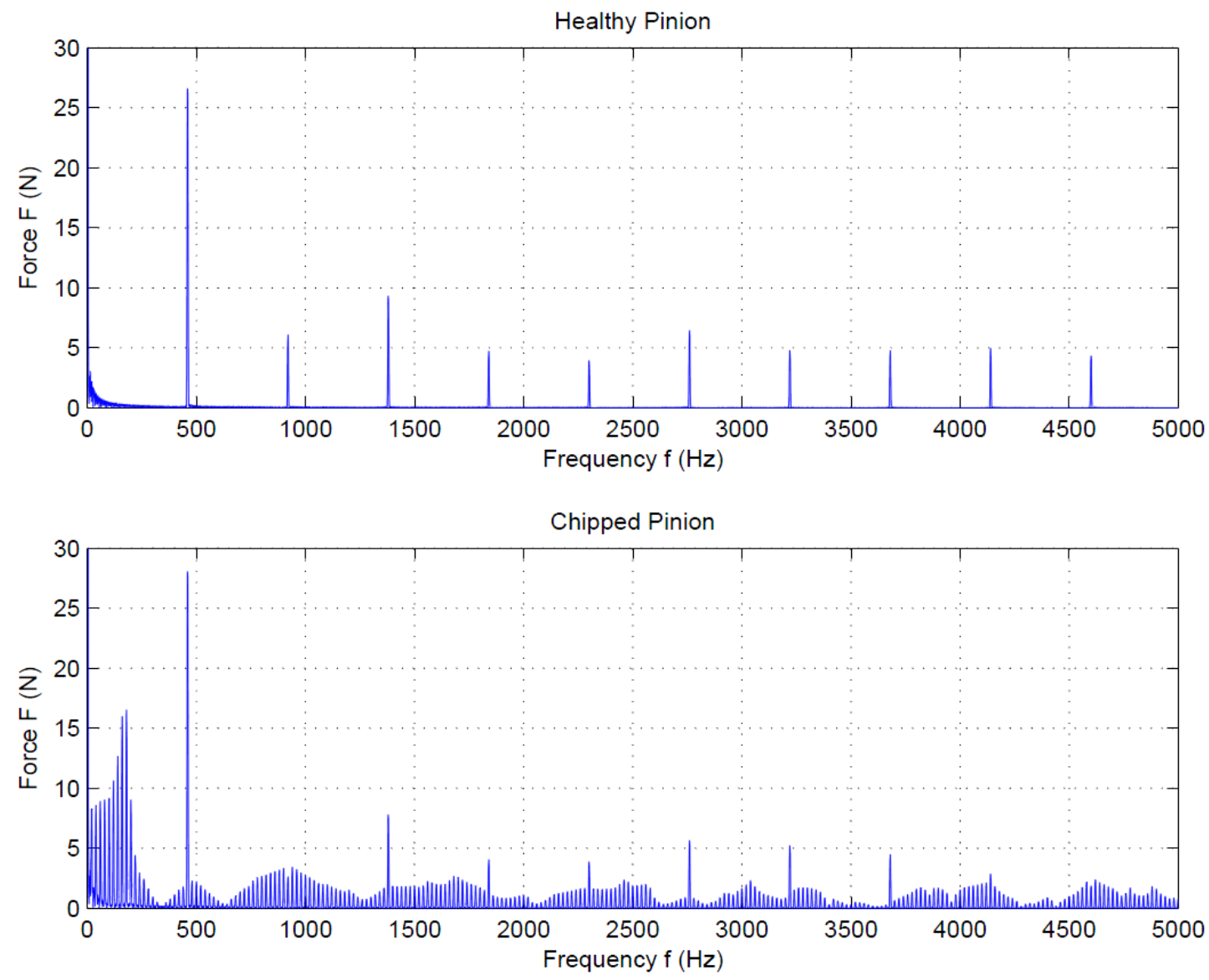

Figure 4.9. GMF of the gearing system at a constant speed of $20 \mathrm{~Hz}$

\subsubsection{Cracked Pinion}

The contact force of a cracked pinion is plotted in the time domain in Figure 4.10. The time domain plot of the cracked pinion contains more noise than the original one, which includes a healthy gear set. To emphasize this, the peaks of the cracked gear are denser. In that case, the time domain signal reveals some information and shows some differences if the contact force of the perfect gearing set is compared. However, a clear identification of a fault in the system cannot be made if the time domain of the contact force is compared only. Thus, once again, the FFT spectrum of this signal is plotted in Figure 4.11. 


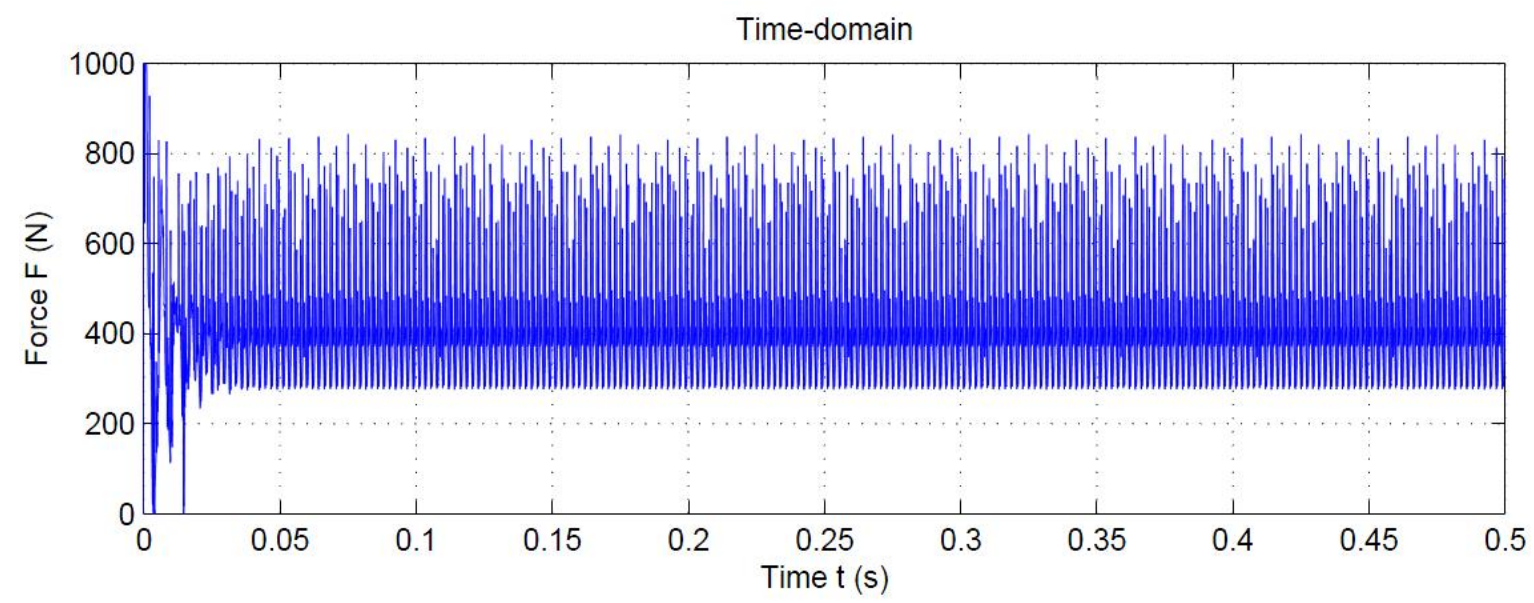

Figure 4.10. Contact force in the time domain of a cracked pinion

As shown above, the gear mesh frequencies show up as peaks in the FFT plot. However, the frequency spectrum contains less noise than the FFT of the chipped gear system. It is difficult to tell if any noise is present in the vibration spectrum due to the cracked gear and therefore it makes a fault detection difficult, especially if the presence of noise in a practical system is higher. Thus, a comparison between the noise at different speeds and setups is necessary in order to fully reveal and identify a crack in the system.

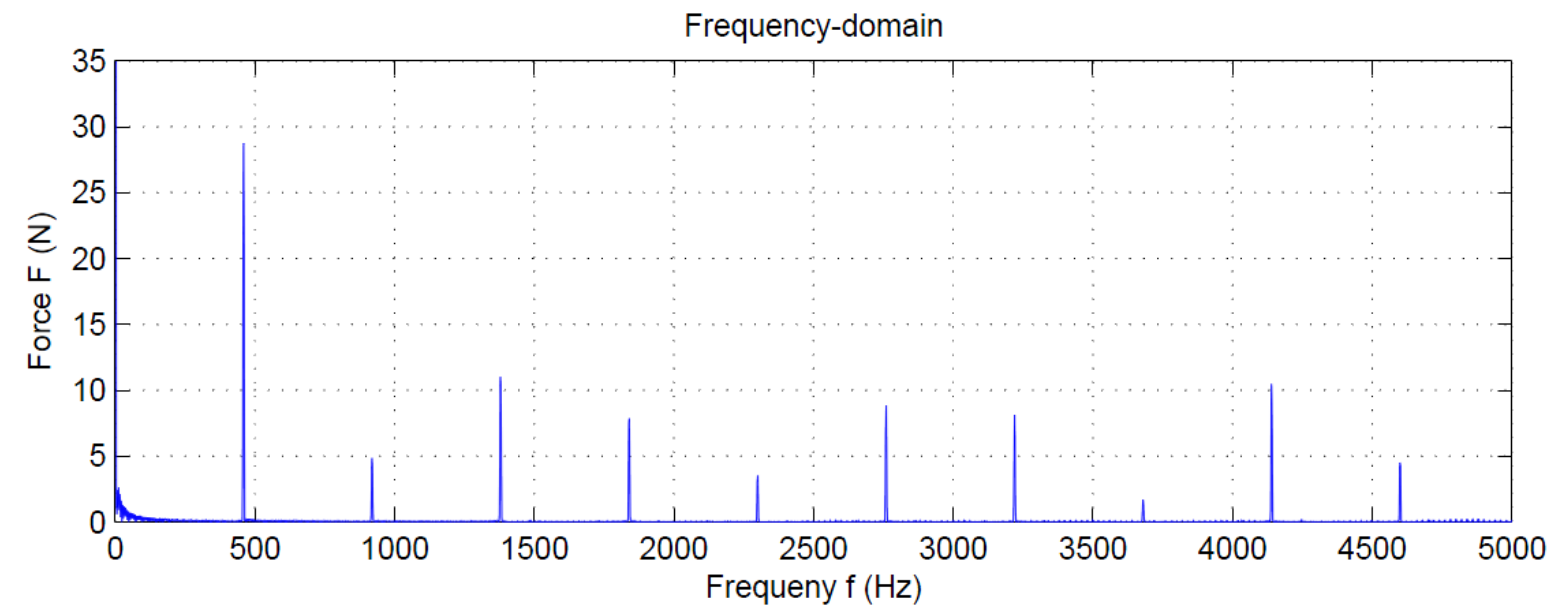

Figure 4.11. Contact force in the frequency domain of a cracked pinion 


\subsubsection{Cracked Shaft}

Lastly, the response of the system with a cracked shaft is documented and presented in Figure 4.12. As can be seen, the vibration response is similar to the other plots. The amplitude of the contact force in the time domain function has approximately the same value as the previous graphs. However, the structure of the signal is different. It shows some irregularities with a structure that appears as a waveform. The reason for that is the cracked in the shaft that increases the oscillating motion of the shaft. If the FFT spectrum is analyzed, you will notice that $20 \mathrm{~Hz}$-modulated sidebands are present on each side of the gear mesh frequency peaks. Therefore, the appearing sidebands in the FFT plot can be an indicator for a crack in the shaft. It is important to emphasize that the contact force in this study is taken to measure to response and that the contact force reveals the irregularities of the shaft. Thus, the contact force can reveal information of bodies that are not in direct contact with gears, such as the shaft.
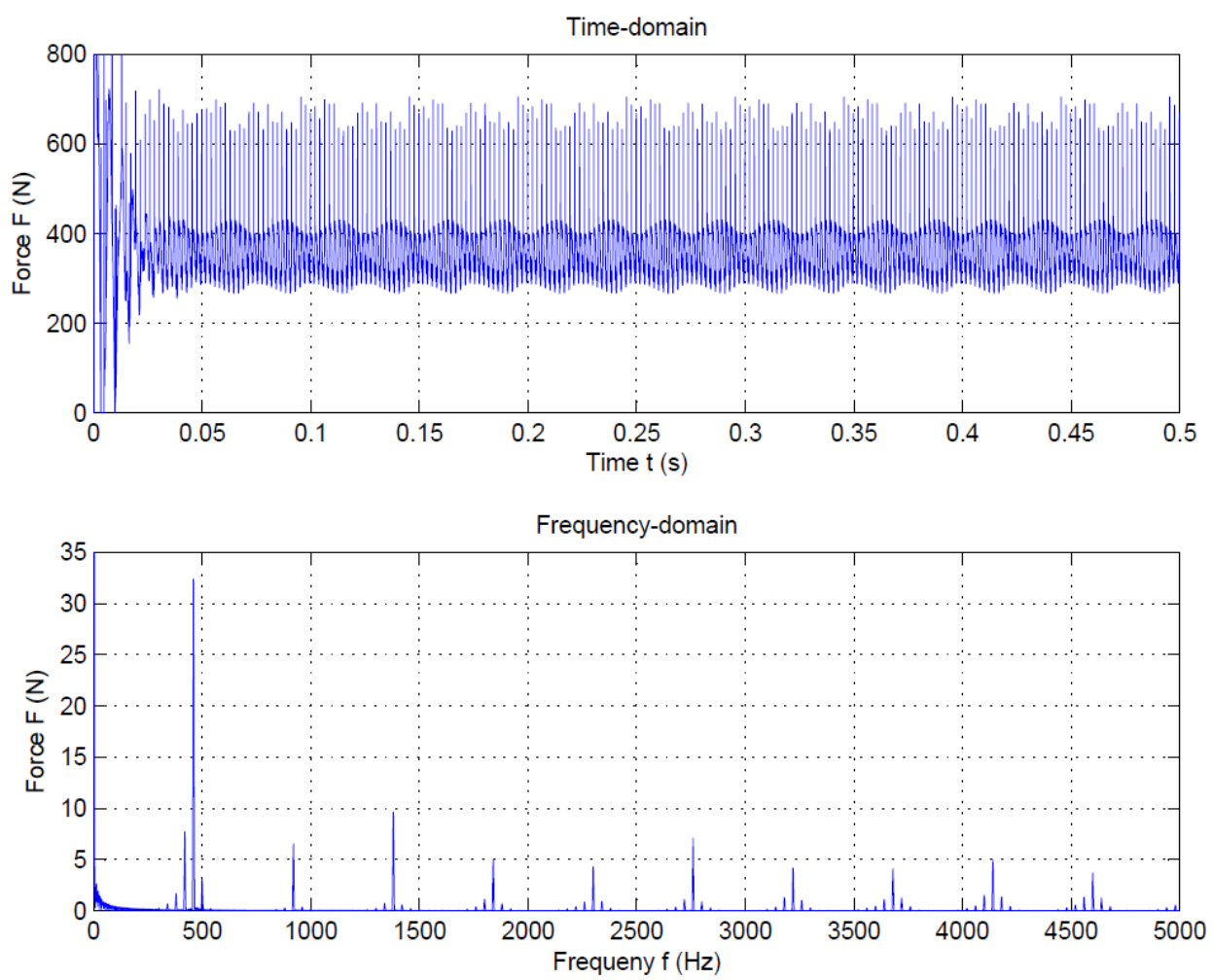

Figure 4.12. Response of the system with a cracked shaft 
The result and summary of the individual faults is shown in Figure 4.13. Note the presence of the sidebands in the individual systems that occur as noise in the FFT.

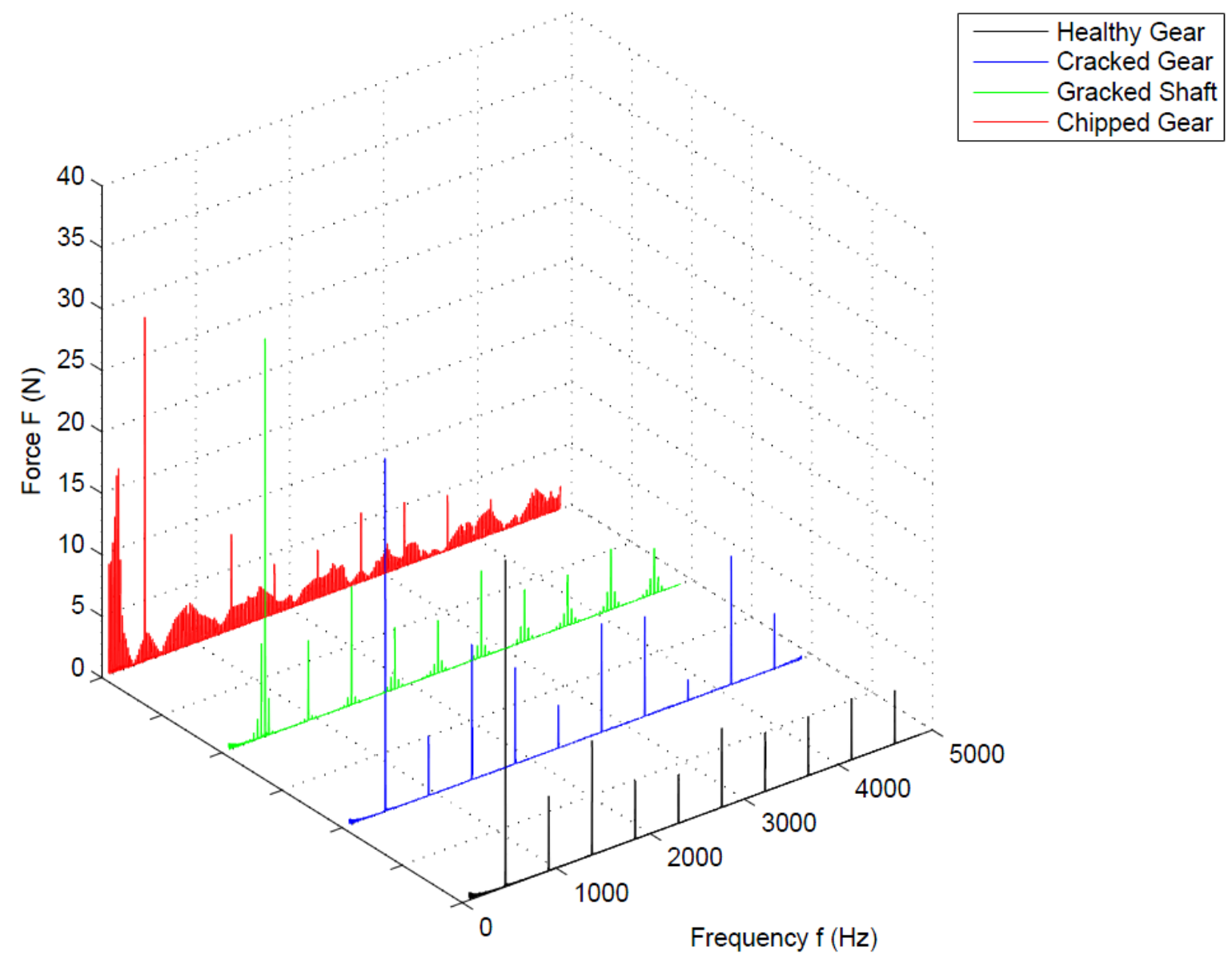

Figure 4.13. Comparison of the faults in the frequency spectrum 


\subsubsection{D FFT Analysis with a Constant Speed}

\subsubsection{Chipped Pinion}

As mentioned before, 2D FFT has its limitations and cannot fully reveal the time information of a signal. However, in many cases it is also important to plot the 3D FFT frequency response, especially if the signal is not constant over time. For this purpose, the fault detection method is applied using the 3D FFT. First with a constant speed of $20 \mathrm{~Hz}$ and then with a linear increasing speed.

Figure 4.14 shows the 3D FFT of a perfect gearing system with a constant speed at $20 \mathrm{~Hz}$. As can be seen, the gear mesh frequencies appear as constant peaks along the time axis. This is due to the fact, that the rotation speed of the shaft is constant and does not vary over time. Note also that almost no noise is present in the entire plot, which indicates that the system is healthy.

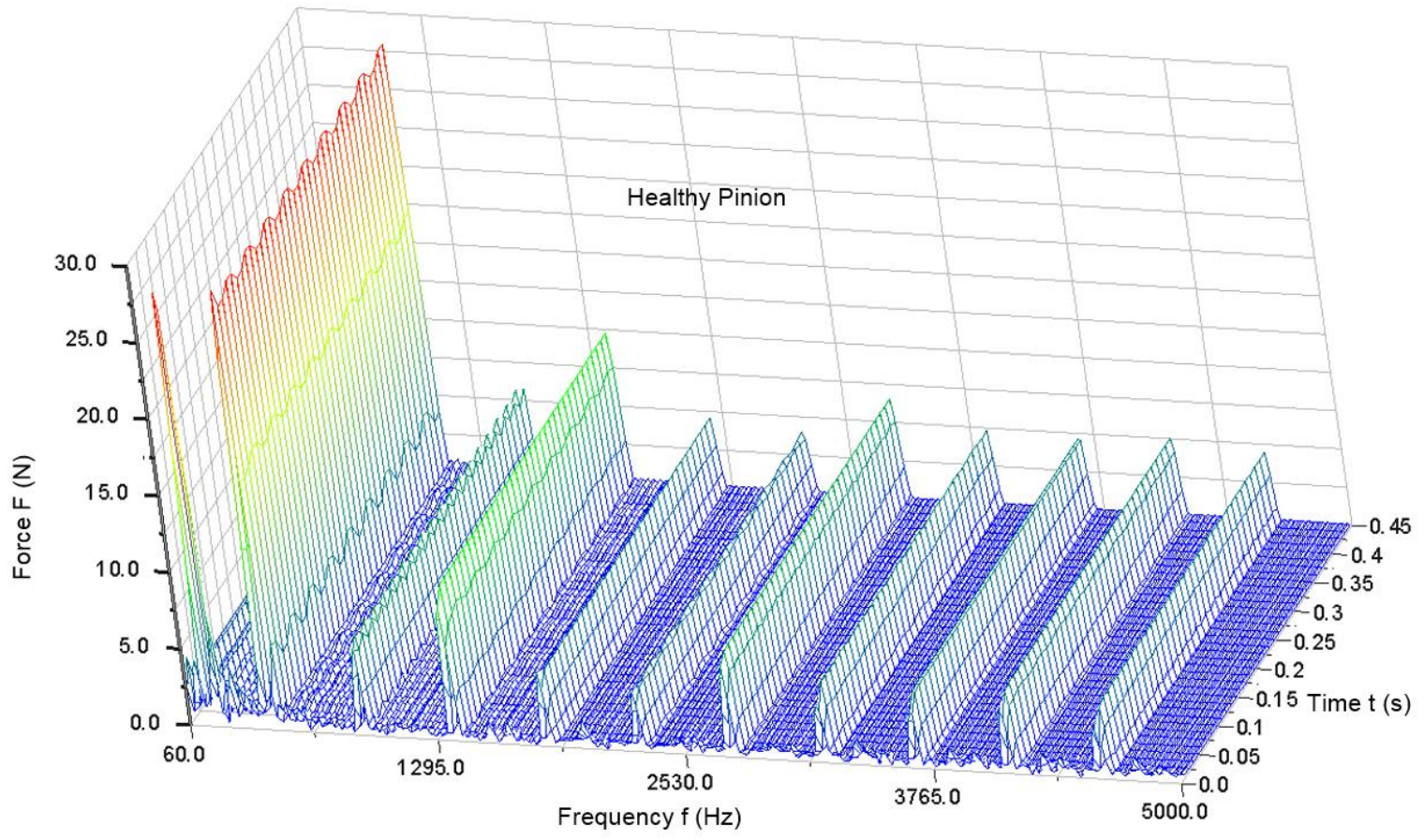

Figure 4.14. 3D FFT of a healthy pinion with a constant speed at $20 \mathrm{~Hz}$ 
To have a comparison to a damaged gear system, the 3D FFT response of a chipped gear is plotted in Figure 4.15. The system contains the same inputs, such as a constant speed at $20 \mathrm{~Hz}$ and the torque profile.

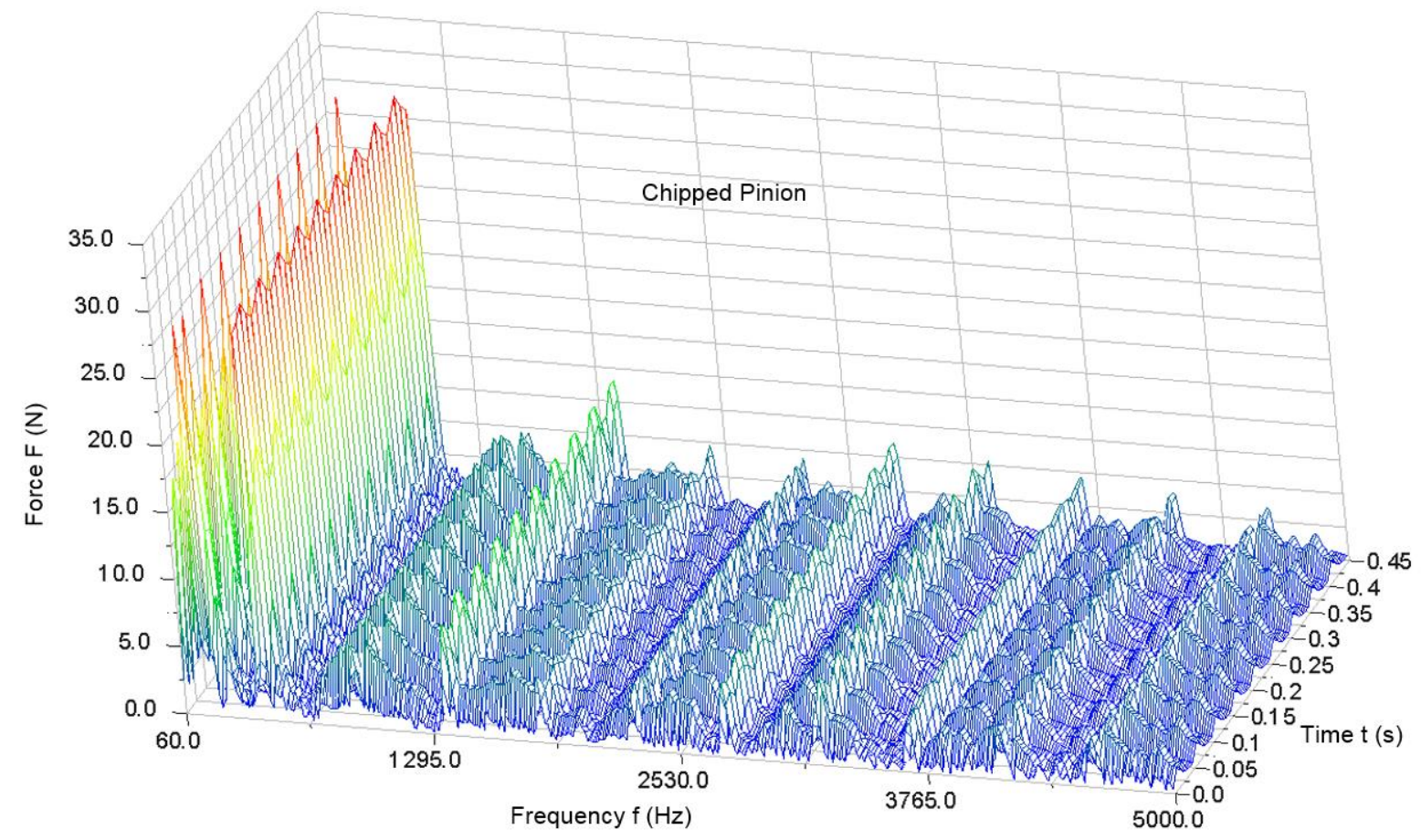

Figure 4.15. 3D FFT of a chipped pinion with a constant speed at $20 \mathrm{~Hz}$

Compared to the perfect pinion, the chipped pinion contains a lot of noise, which is present over the whole time range. Moreover, the magnitude of the gear mesh frequency peaks do not show a constant distribution over the time axis. In particular, the peaks are oscillating slightly, which means that at certain intervals $(0.05 \mathrm{~s})$ the peaks reach a slightly higher magnitude. This fact, as well as the heavy presence of noise in this plot, is an indicator that the system has a damaged tooth. Therefore, 3D FFT is a suitable method to detect chipped gears in a gearing system. 


\subsubsection{Cracked Shaft}

The next analysis includes the 3D FFT plot of a cracked shaft. The response of this damaged system is presented in Figure 4.16.

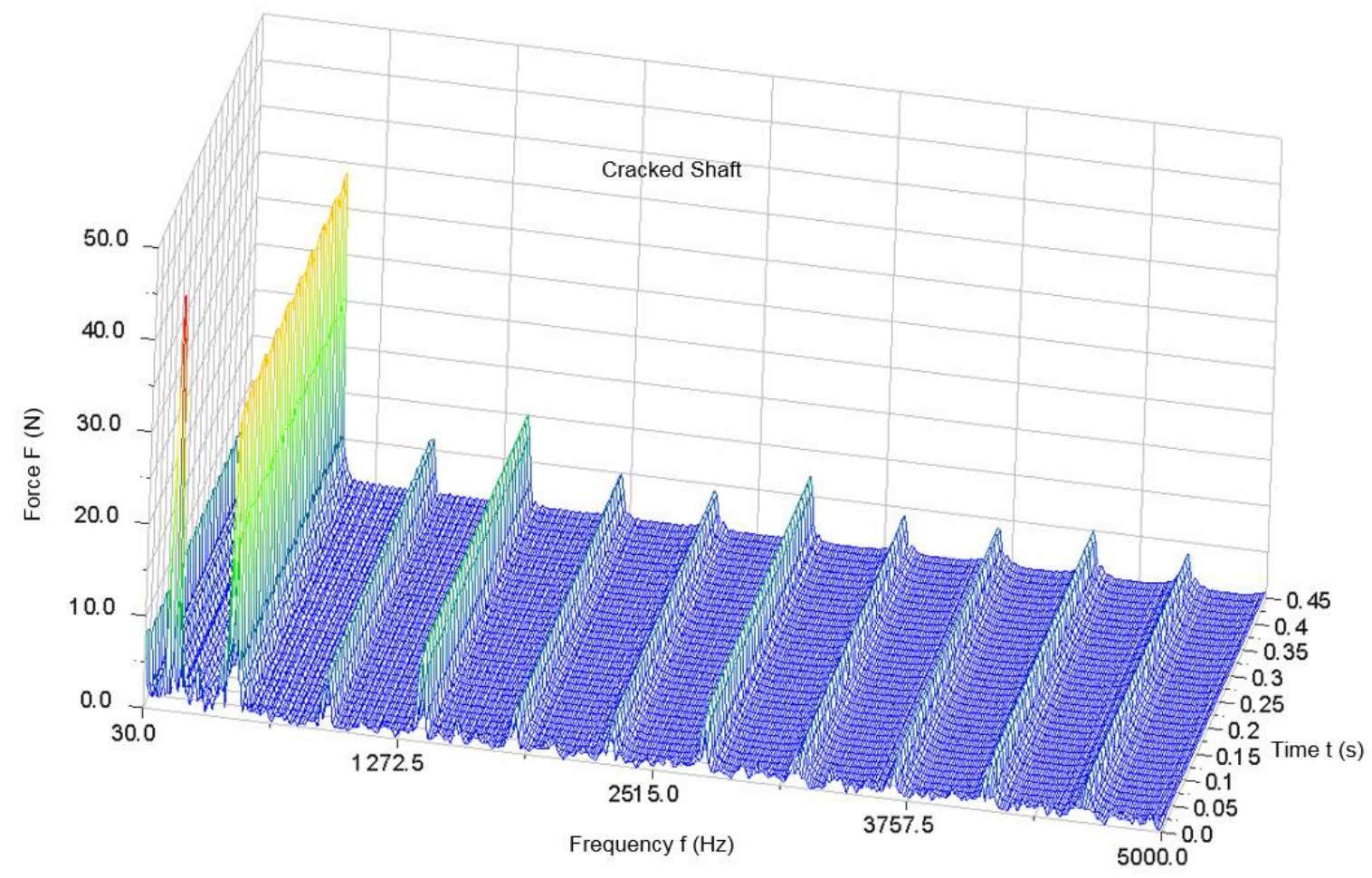

Figure 4.16. 3D FFT of a cracked shaft with a constant speed at $20 \mathrm{~Hz}$

Surprisingly, the response shows similarities to that one of a healthy gear. A clear noise present is non-existing and it is difficult to tell if there is any fault in the system. Even the noise appearance is similar to the healthy gear system.

\subsubsection{Cracked Pinion}

Finally, the three-dimensional frequency spectrum of the cracked pinion with a constant speed at $20 \mathrm{~Hz}$ is shown in Figure 4.17. Note that the plot is similar to the plots with the healthy gear and the cracked shaft. As you can see, the frequencies as well 
as the amplitude are almost the same. There are not many visible difference between the individual plots and a clear identification of a crack in the pinion cannot be made.

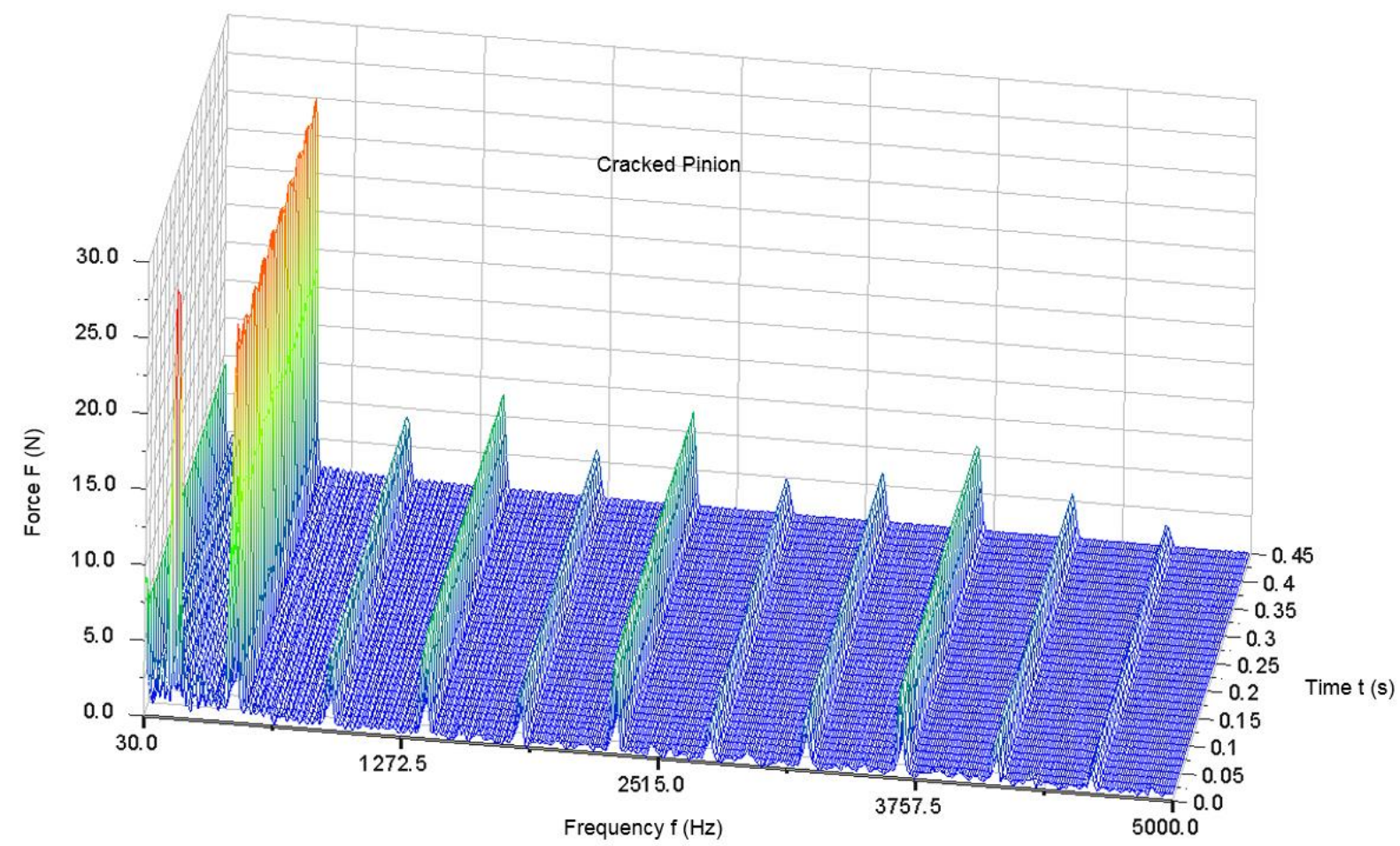

Figure 4.17. 3D FFT of a cracked pinion with a constant speed at $20 \mathrm{~Hz}$ 


\subsubsection{Linearly Increasing Speed Analysis}

The next research objective is the analysis of the time domain and 3D FFT of the system with a linearly increasing speed profile. The model is subjected to a driving speed at the input shaft with a linearly increasing function $(0-400 \mathrm{rpm})$. As the speed increases, the contact force increases as well, which is shown in Figure 4.18. Furthermore, the time domain signal shows high vibration peaks that appears whenever the damaged gear is in contact.
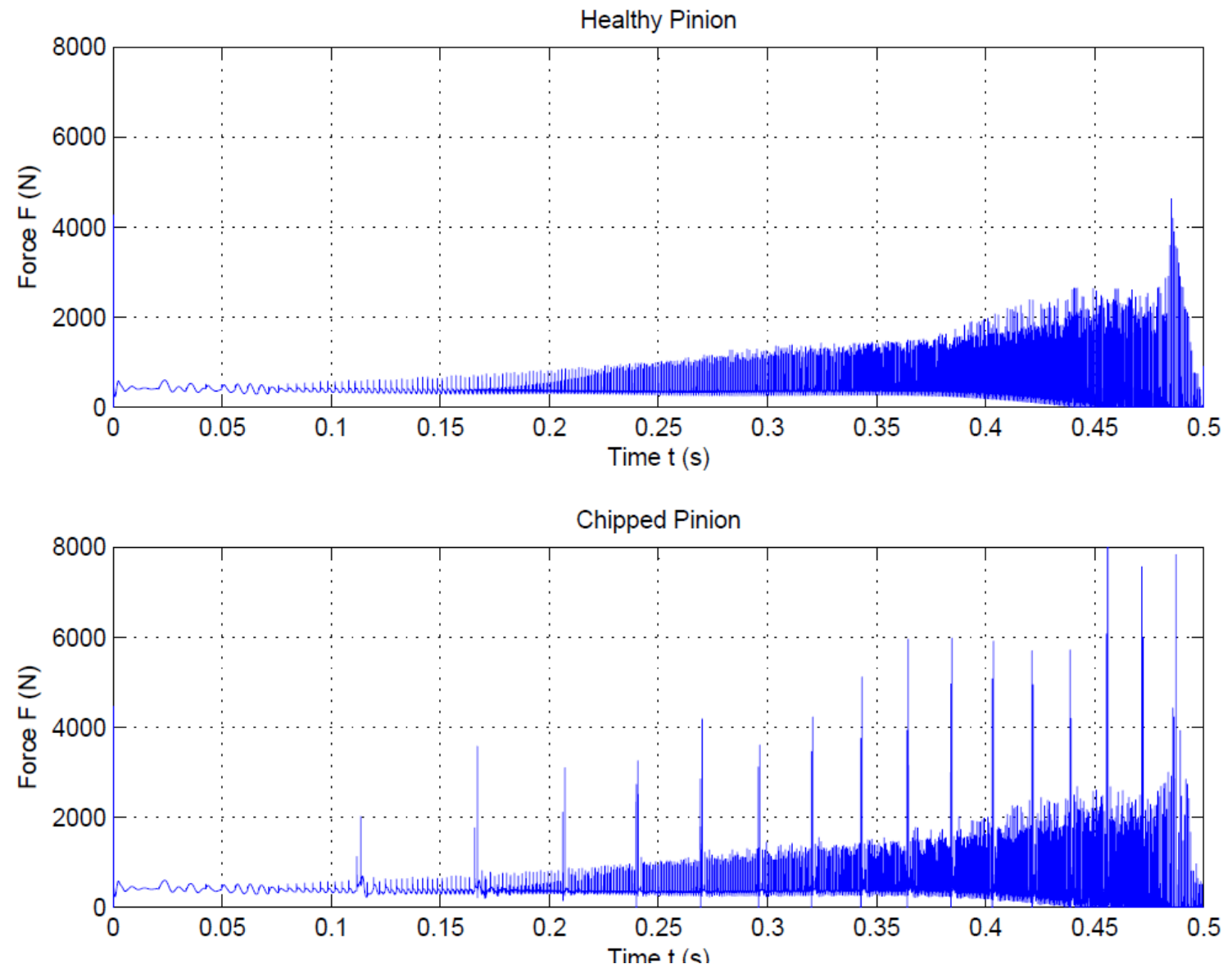

Figure 4.18. Time domain of a healthy and a chipped pinion with an increasing speed

As expected, the FFT does not show any reasonable results for both, the chipped pinion and the healthy pinion, since the input speed does not create any periodic contact force between the two gears. Nevertheless, it shows some differences that can be 
noticed by the noisy form of the FFT plot in Figure 4.19. However, it is not a reliable method for fault detection and should not be used to analyze the systems' response.
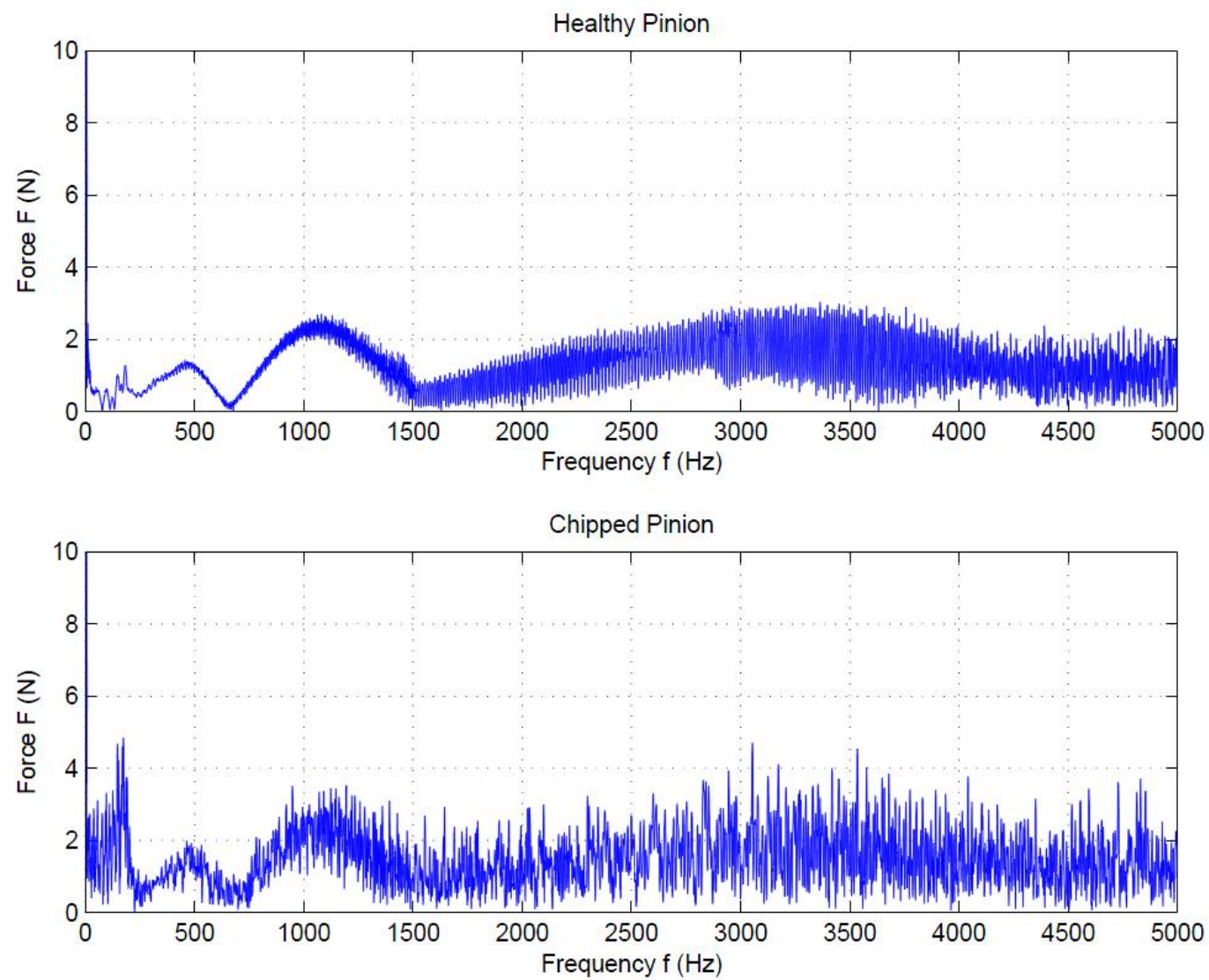

Figure 4.19. Frequency domain of a healthy and a chipped pinion

A better alternative is to focus on the 3D FFT for non-constant speeds in order to gain a better understanding of the response, which is shown in Figure 4.20. As you can see, the gear mesh frequencies are present as a "waterfall plot" in form of waves. As the speed increases, the gear mesh frequencies shift along the frequency axis and create such a unique plot. However, the chipped pinion shows a higher noise profile in the 3D FFT plot than the 3D FFT of a healthy pinion. Furthermore, the peaks of the wave show irregularities over the time. Refer to the appendix to see the results for the exponential speed profile. 

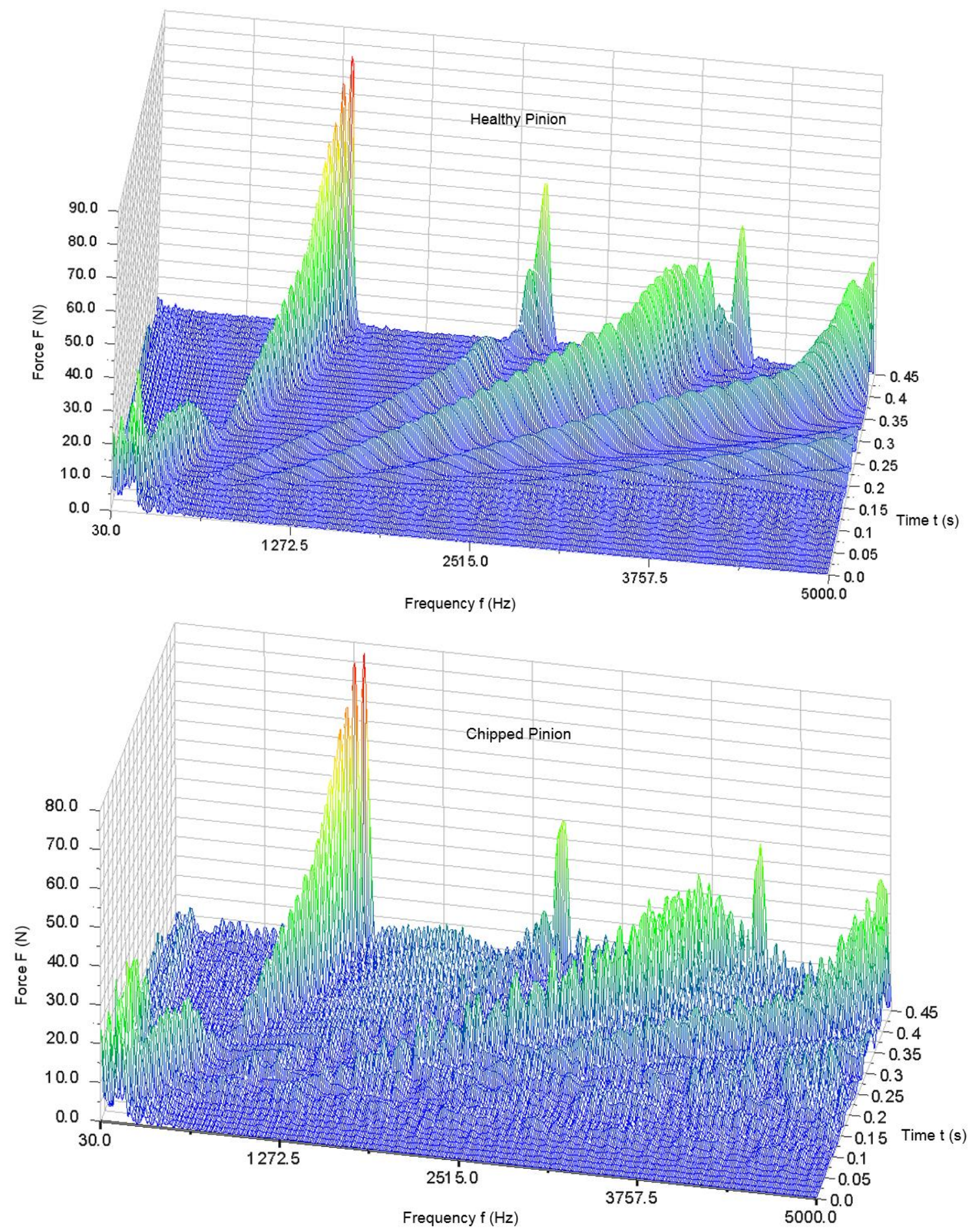

Figure 4.20. 3D FFT of chipped and healthy gear subjected to an increasing speed

It is also possible to analyze the 3D frequency domain plot of a cracked shaft subjected to a linearly increasing speed. Note that the differences lie at the end of the GMFs peaks that appear as increasing lines. The GMFs show a different waveform at the at 
the end of the time. Thus, 3D FFT is a suitable method to detect a cracked shaft for his model.

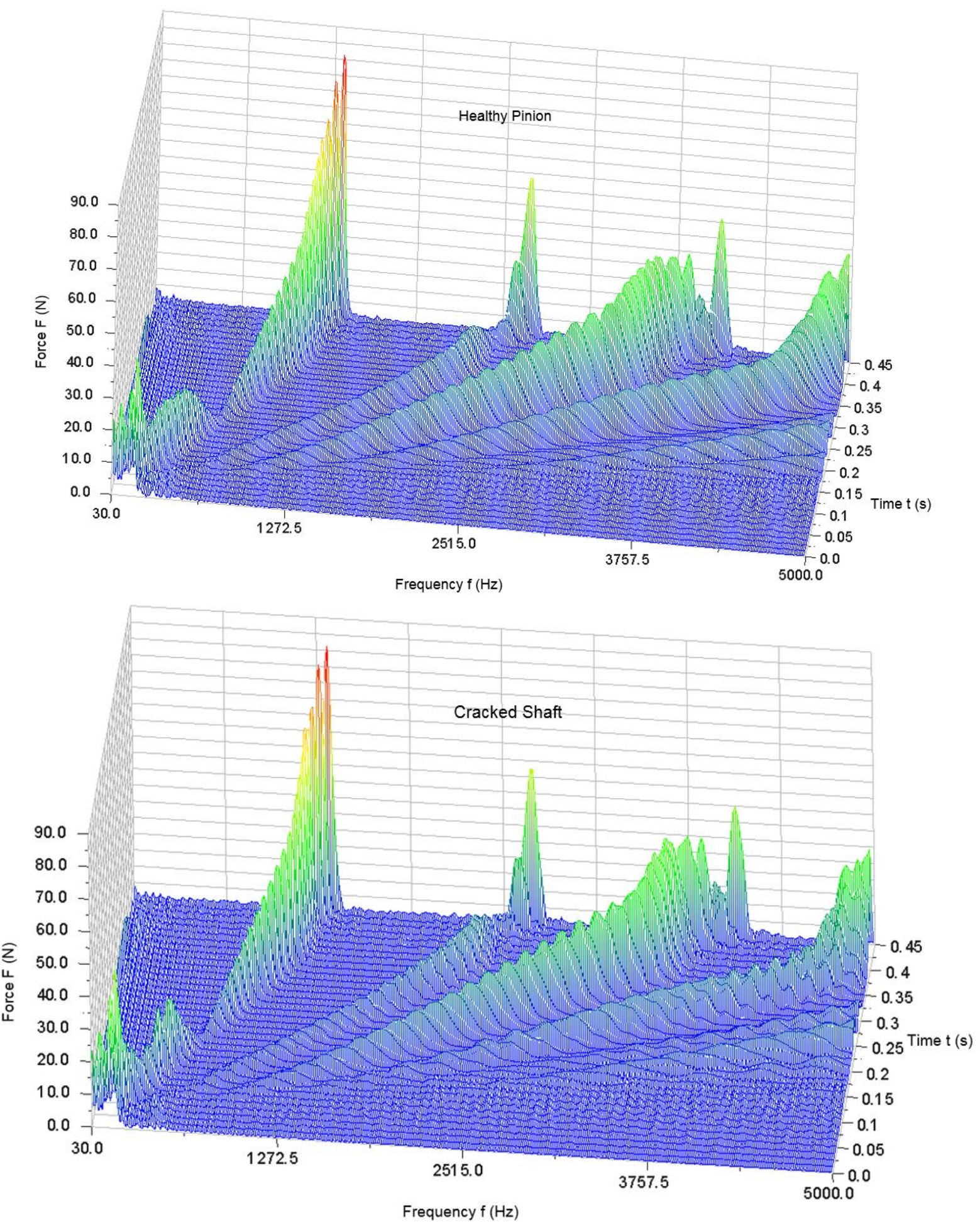

Figure 4.21. 3D FFT of cracked shaft and a healthy gear with an increasing speed 

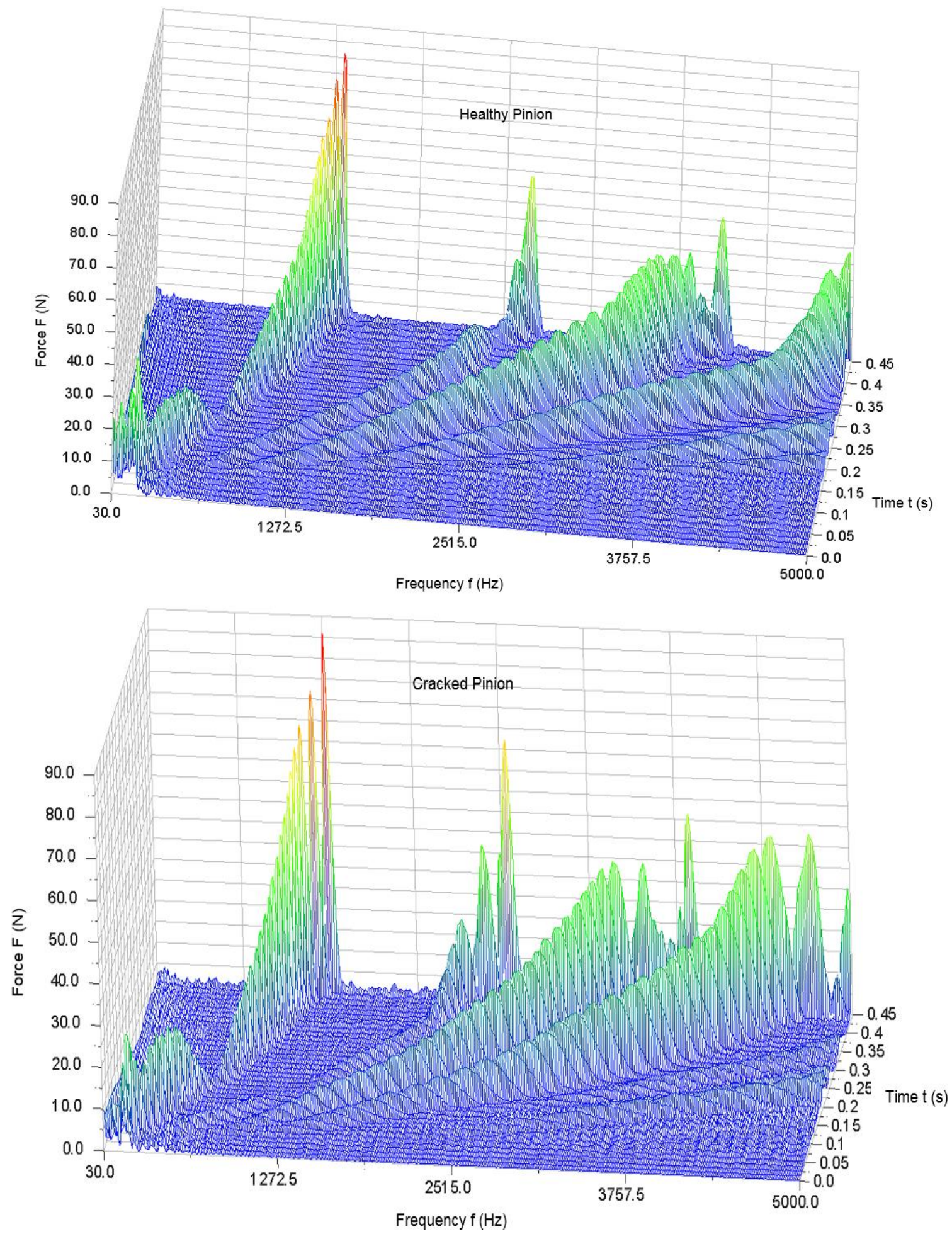

Figure 4.22. 3D FFT of cracked pinion and a healthy gear set with an increasing speed

Finally, the 3D FFT plot of a cracked pinion is plotted and compared in Figure 4.22. Notice that the lines of the GMF's peaks for the cracked pinion are higher than the peaks of a healthy gear, which is an indicator for a fault in the system. 


\subsection{Wavelet Analysis}

As previously mentioned, FFT analysis is limited for the use in fault detection. 3D FFT has also its limitations regarding the time and frequency resolution. To overcome this drawback, the Wavelet Transform will be used for health monitoring in this chapter. The same settings and the model setup, which were used in the previous analysis are used for the next analysis. The contact force of the gearing system is recorded in Adams and exported to Matlab, where the Wavelet Toolbox is used in order to perform the Continuous Wavelet Transform with the Wavelet function bior3.3.

\subsubsection{Wavelet Analysis of a Healthy Gear}

The first Wavelet plot includes the contact force of a healthy gear set. As seen in Figure 4.23, the $\mathrm{x}$-axis of the plot (for the signal as well as the Wavelet) is presented in terms of scales. This variable stands for the number of samples of the signal and has a total number of about 50,000 . However, the number of samples relates to the time. For instance, a simulation time of $0.5 \mathrm{~s}$ is set to perform the analysis in Adams with 50,000 samples. Thus, they are linearly related and presented in Figure 4.23. 


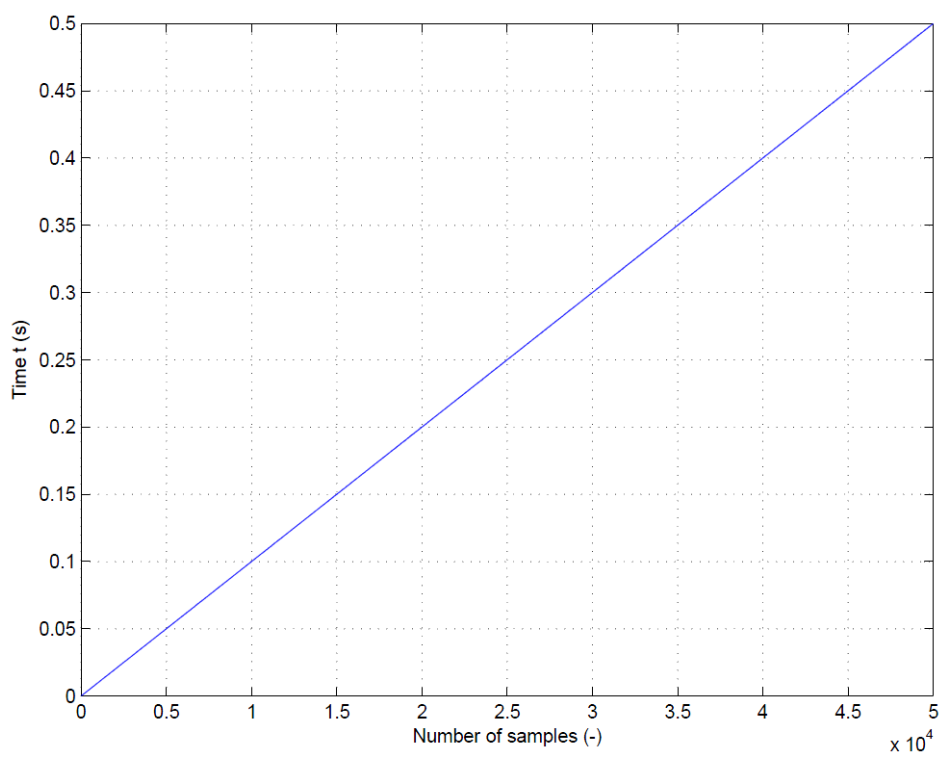

Figure 4.23. Relation between the number of samples and the time

On the contrary, the y-axis of the Wavelet plot represents the number of scales. As previously mentioned in the introduction, the scales relate to the frequencies. Several high-energy densities occur as colored straight lines in the wavelet plot. These lines represent the gear mesh frequencies and can be converted to real frequencies using Figure 4.24. For example at a scale of 205 the correspondence frequency is $460 \mathrm{~Hz}$, which is the first gear mesh frequency of the system.

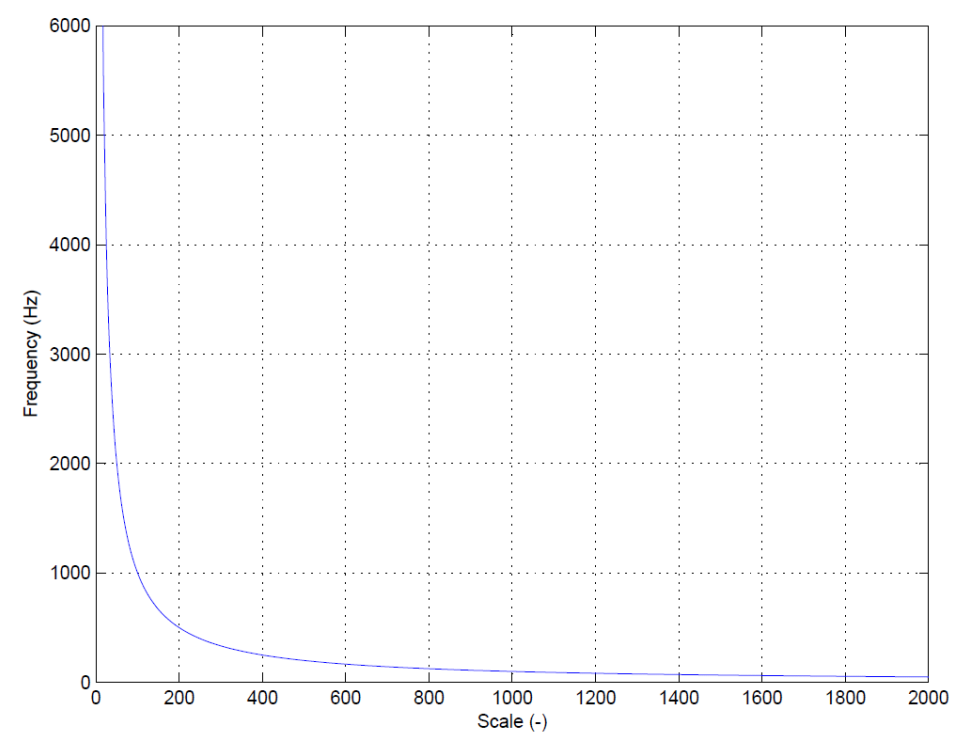

Figure 4.24. Correspondence frequency to scale table 


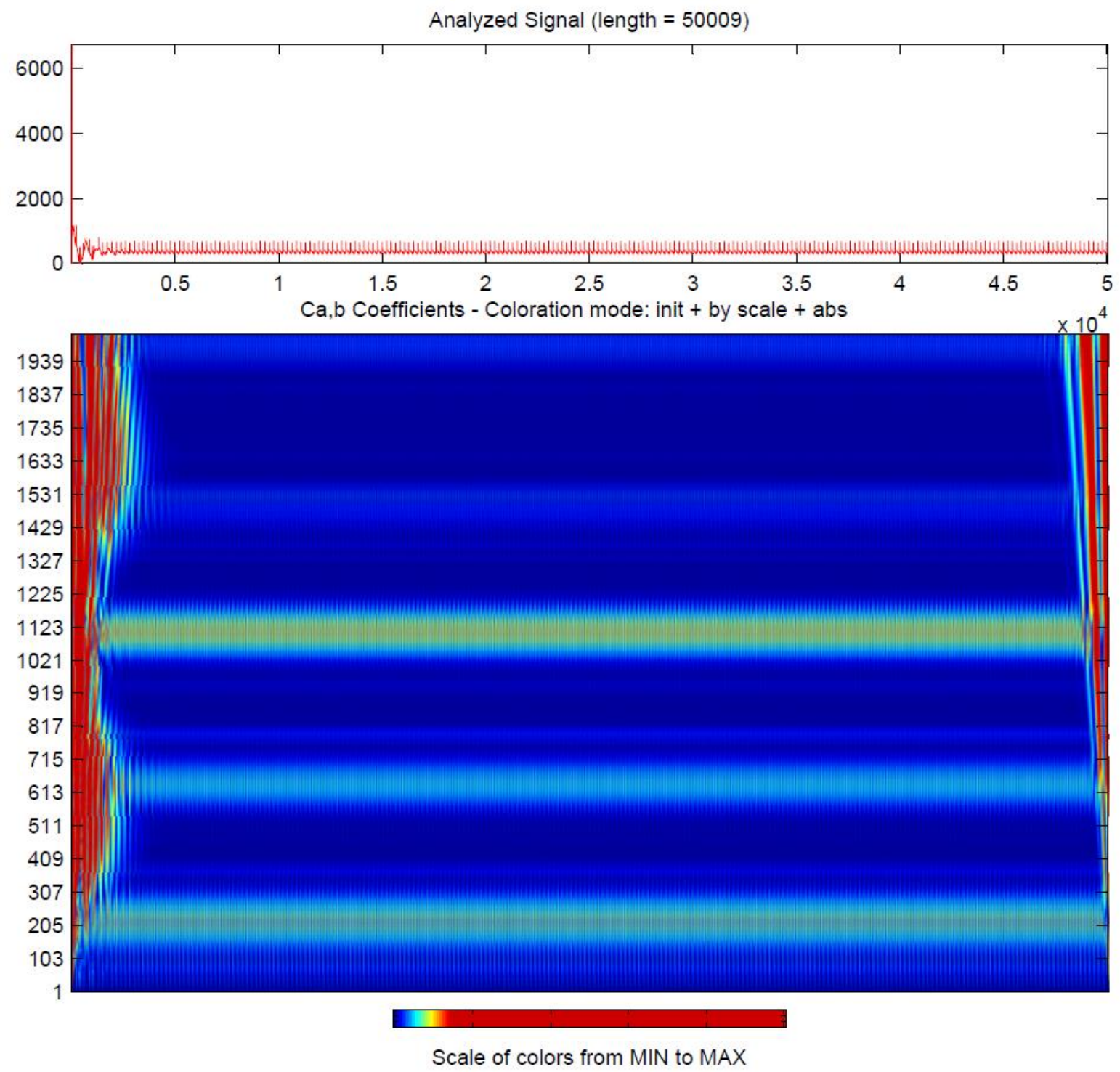

Figure 4.25. Wavelet analysis of a healthy gear system with a constant speed at 20 $\mathrm{Hz}$

Unfortunately, the plot does not reveal the information of how big the amplitude of the response is. It only shows where the high-energy densities occur. The constant lines do not change over the time range and do not show any irregularities, which leads to the conclusion that the system is healthy and does not contain any faults. However, more analysis have to be carried out in order to identify defects in systems. 


\subsubsection{Wavelet Analysis of a Chipped Pinion}

The next analysis includes the Wavelet Transform of a chipped gear, which is shown in Figure 4.26. As you can see, the structure of the wavelet plot differs from that one with the healthy gear system. In addition to the constant lines, several perpendicular shapes appear in the Wavelet plot. This happens whenever the damaged gear is in contact. Hence, a clear detection of a damaged gear can be made if Wavelet Transform is considered. Furthermore, the location of the damaged tooth can also be revealed, which is another advantage using this method.

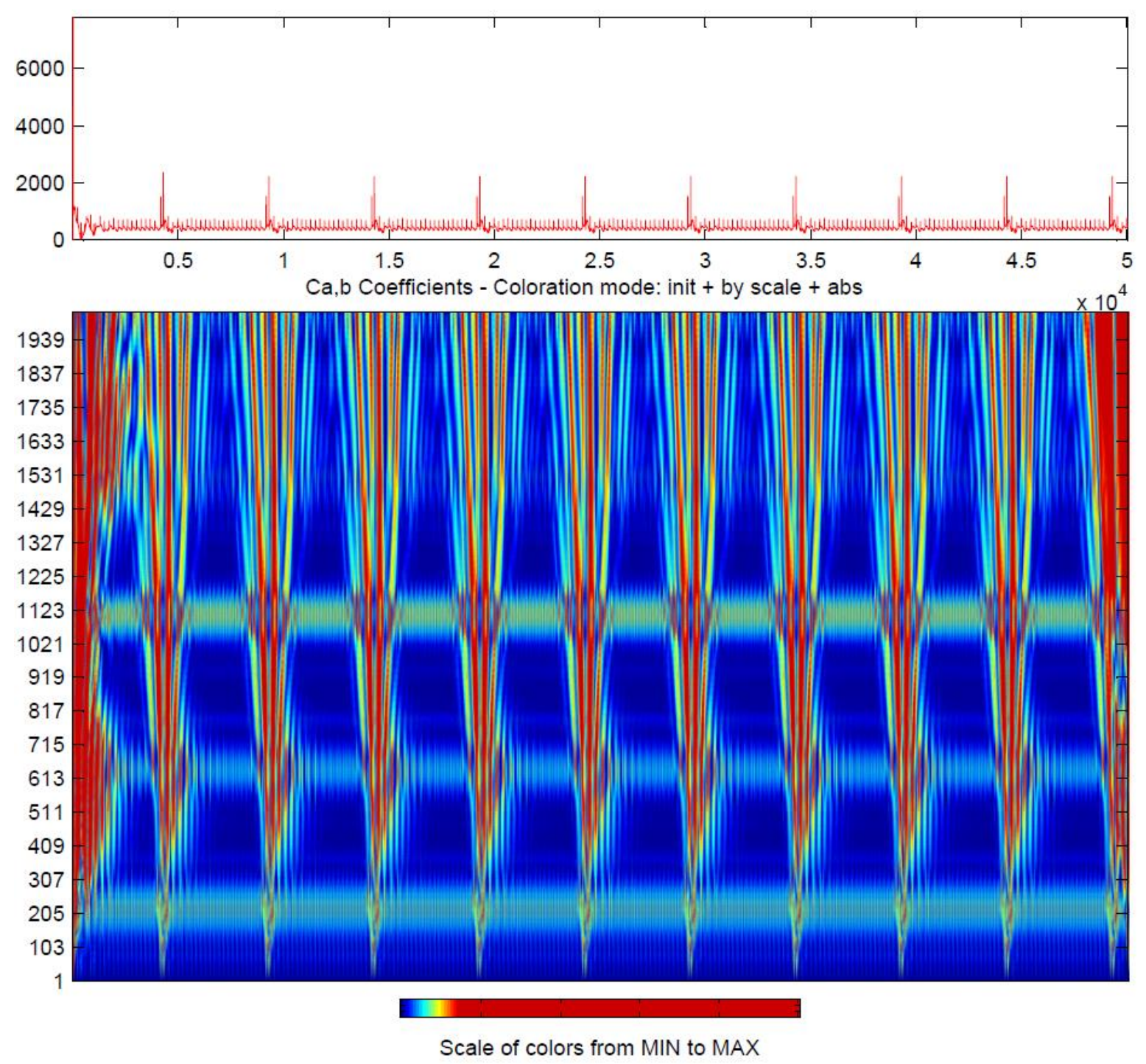

Figure 4.26. Wavelet plot of a chipped pinion with a constant speed at $20 \mathrm{~Hz}$ 


\subsubsection{Wavelet Analysis of a Cracked Shaft}

The next analysis contains the vibration analysis of cracked shaft using Wavelet Transform. The result is plotted in Figure 4.24. As can be seen, the vibration pattern differs from the previous plots. It shows an oscillating structure of the peaks and is due to the cracked shaft that causes this pattern to oscillate.

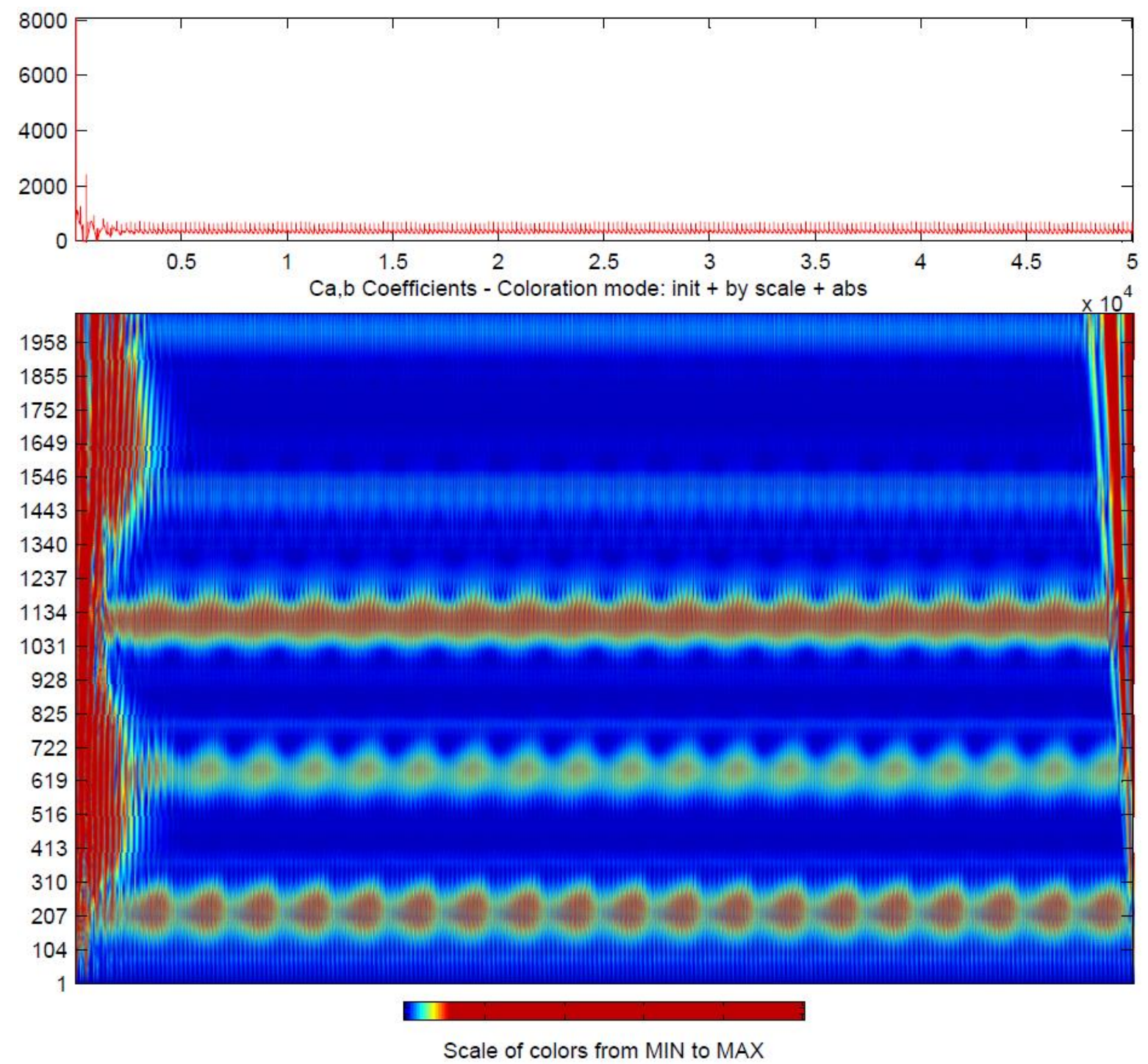

Figure 4.27. Wavelet plot of a cracked shaft with a constant speed of $20 \mathrm{~Hz}$ 


\subsubsection{Wavelet Analysis of a Cracked Gear}

The last analysis includes the Wavelet plot of a cracked gear, which is presented in Figure 4.28. As can be seen, this Wavelet plot is similar to the Wavelet plot with the healthy gearing system. However, the constant lines show some inhomogeneity over the time axis. This can be an indicator that a crack in the gear is present. However, it is not reliable enough to make a clear fault identification, since the source of this phenomenon could also be the cause of noise. However, if another speed profile is taken into account, the crack can be identified using Wavelet transform. See the appendix for details.
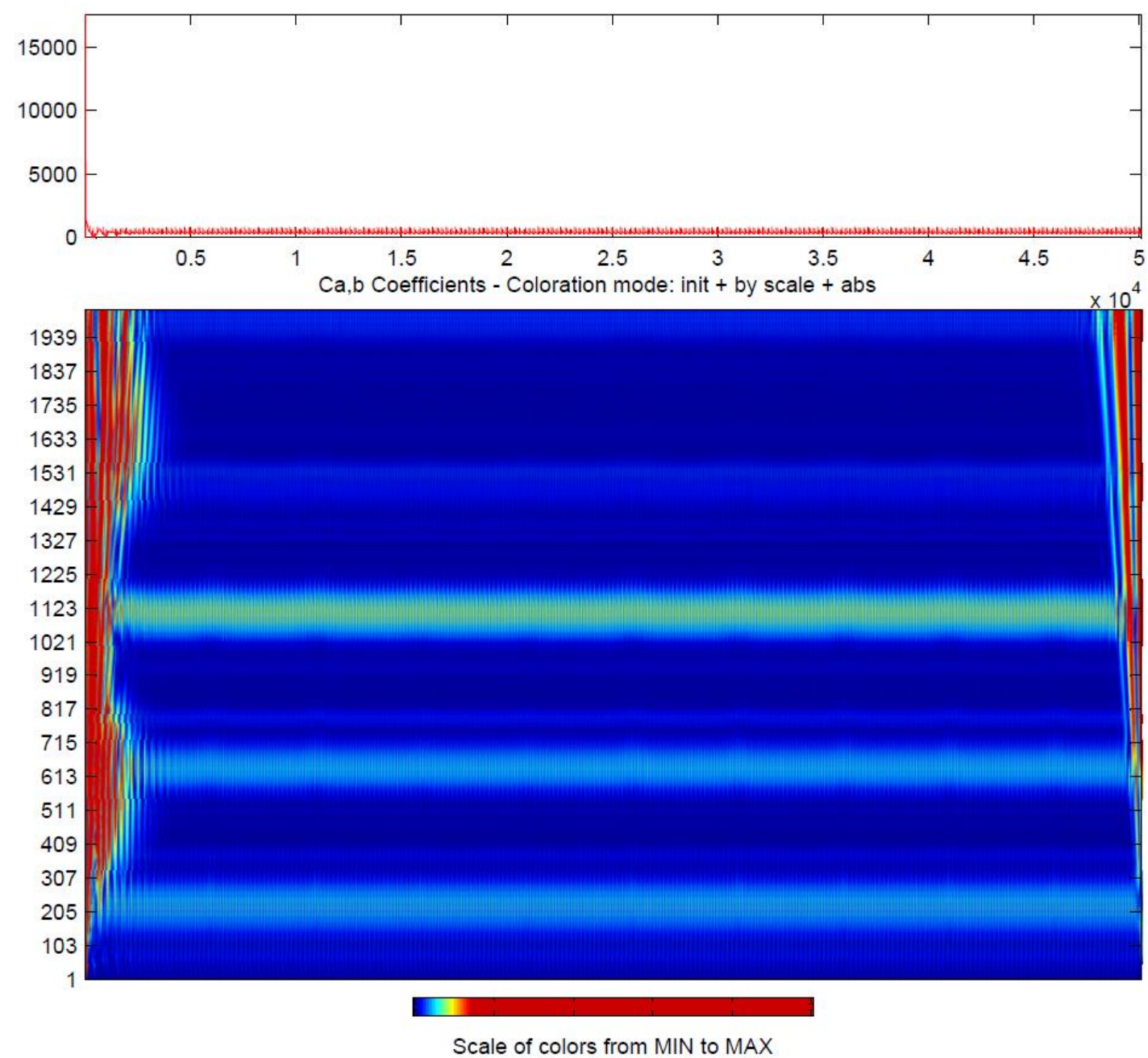

Figure 4.28. Wavelet plot of a cracked pinion with a constant speed at $20 \mathrm{~Hz}$ 


\subsection{Statistical Fault Detection Methods}

This section focuses on health monitoring using statistical methods. As a contrary to the visual detection methods, like Wavelet and 3D FFT, statistical methods could be an approach to find faults with an automated algorithm. Therefore, the standard deviation, the mean and the root mean square, as well as the Side Band Energy Ratio, will be presented and discussed in this chapter. Furthermore, they will be applied in order to display the differences between the individual faults in the gear set. The same faults (chipped pinion, cracked pinion and cracked shaft) will be used in this chapter and the statistical methods will be applied for different constant speed values $(10-60 \mathrm{~Hz})$ in order to reduce the possibility of random noise. The statistical methods will be used in order to calculate the time domain signal and the noise presence of the signal in the frequency domain.

\subsubsection{Root Mean Square (RMS)}

One of the simplest method in health monitoring is applying the root mean square (RMS) to the signal in the time domain. It is suitable to monitor the defects in a system, such as a gearing system, and to include and track the noise. The root means square is also called the quadratic mean and is presented in equation (4.2) [19].

$$
R M S=\sqrt{\frac{1}{N} \sum_{i=1}^{N} x_{i}^{2}}
$$

Where $x_{i}$ correspondences to value of the signal, $N$ for the number of samples of the signal and $i$ stands for the index. 


\subsubsection{The Mean}

The mean value of a signal or data points is also known as the average. The mean is calculated by summing the values of all data points and dividing it by the total number of samples. In many cases, it can be important to compare a set of data points with the mean.

$$
\bar{x}=\frac{\sum_{i=1}^{N} x_{i}}{N}
$$

\subsubsection{The Standard Deviation}

The standard derivation is another statistical method and describes how close a set of data values are to the mean value. Accurate data are present when the data values are close to the mean, which leads to small standard derivation. On the other hand, a large standard derivation will indicate that the data set is spread.

$$
\sigma=\sqrt{\frac{1}{N-1} \sum_{i=1}^{N}\left(x_{i}-\bar{x}\right)^{2}}
$$

\subsubsection{Sideband Energy Ratio (SER)}

Another method for fault detection in gear systems is the usage of Sideband Energy Ratio (SER). As previously shown, a damaged gear system such as a chipped gear, has a greater noise profile in the frequency spectrum, which is present in form of sidebands on each side of the gear mesh frequencies.

SER sums the magnitude of the first six sidebands, and divides it by the magnitude of the center GMF, shown in Figure 4.29. This is done with the first three gear mesh 
frequencies. The equation of SER is shown in equation 4.5. If SER exceeds the value of one indicates a damage in the gearing system [20].

$$
S E R=\frac{\sum_{i=1}^{6} \text { Sideband Amplitude }_{i}}{\text { Center Mesh Frequency Amplitude }}
$$

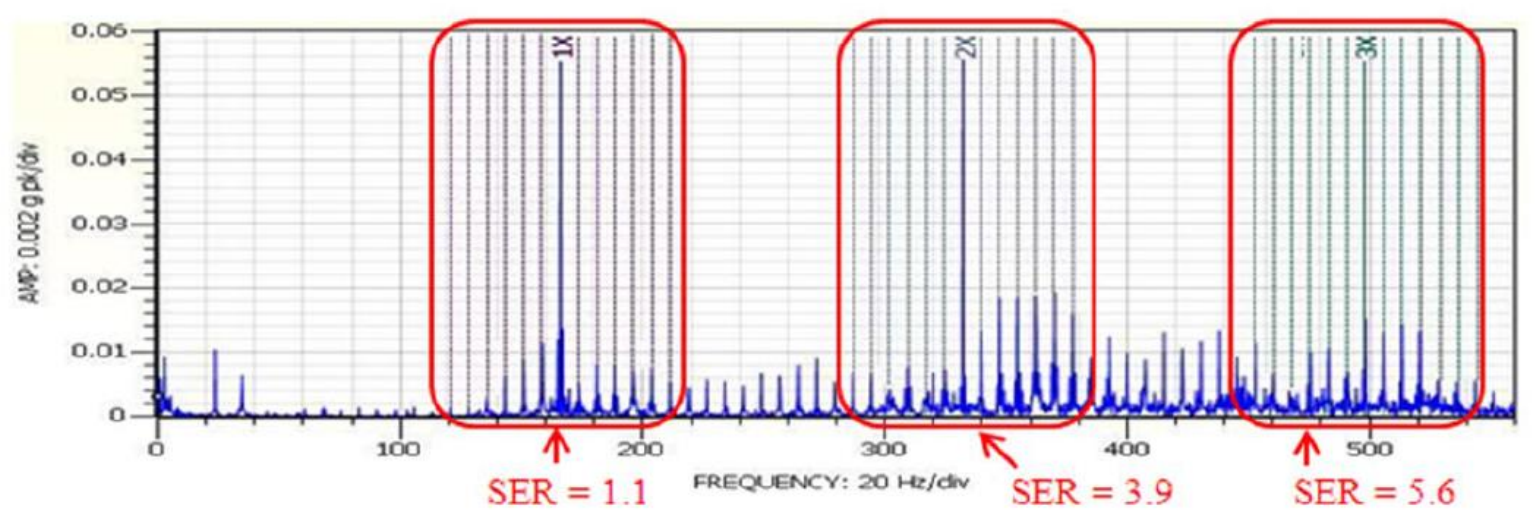

Figure 4.29. SER for the first three gear mesh frequencies [20]

\subsubsection{Statistical Methods for the FFT Spectrum}

Besides using the SER method to detect faults in the gear system, the mean, as well as the standard derivation, will be used to analyze the noise profile in the FFT spectrum. Similar to the work in [21], where the noise of the frequency spectrum was analyzed within two frequency ranges; three areas will be introduced in this paper. 


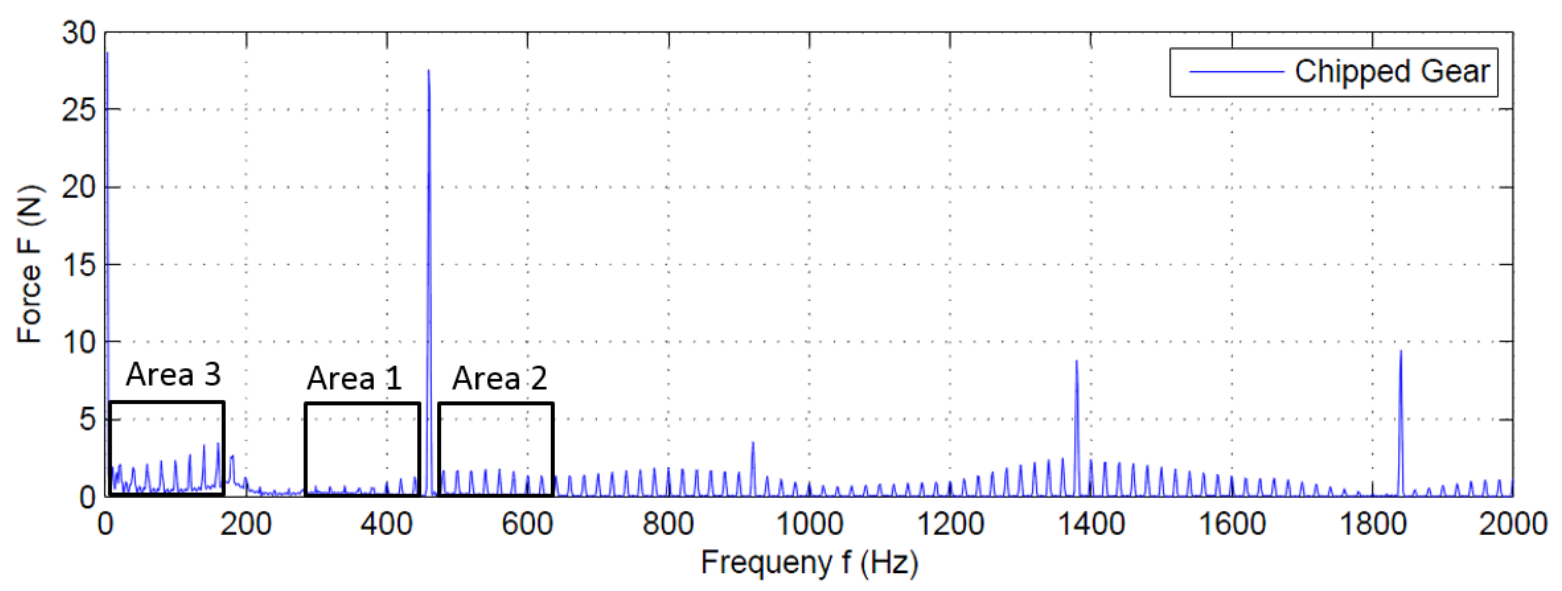

Figure 4.30. Definition of three frequency ranges for fault detection analysis

Figure 4.30 shows the details of the three frequency ranges that will be used in order to calculate the mean and the standard derivation of the noise in the FFT spectrum. Area 3 is placed at the beginning of the FFT plot while the block of Area 1 and Area 2 are placed to the left, respectively to the right of the first gear mesh frequency.

\subsection{Results}

The results of health monitoring using statistical methods that were explained are shown and discussed in this section. First, the results of the analysis of the signal in the time domain will be shown and explained, followed by the results of the analysis in the FFT spectrum.

\subsubsection{Analysis of the Signal in the Time Domain}

The first result is the analysis of the contact force in the time domain, shown in Figure 4.31.The results for the root mean square are presented in the left, while the mean values for the different defects are presented in the right part of the plot. The vertical axis represents the contact force and the horizontal axis reveals the speed, at which the RMS and the mean were calculated. 

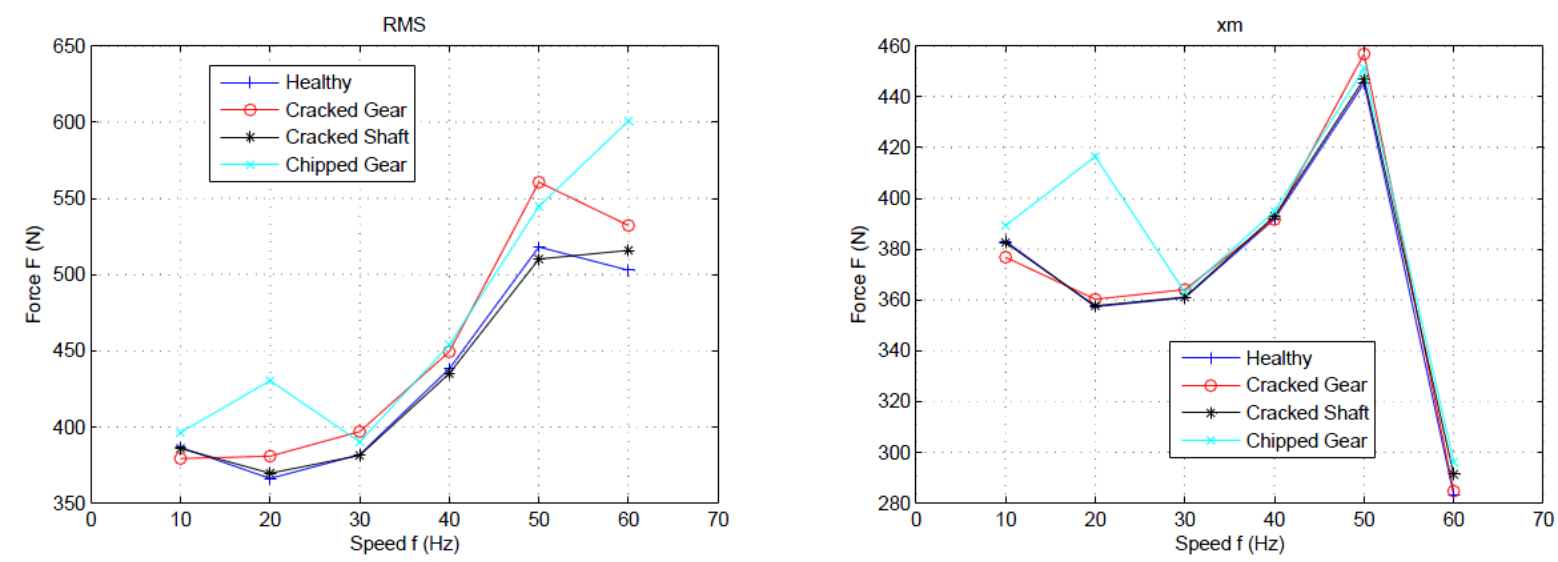

Figure 4.31. RMS and mean values of different faults

It can be seen that the RMS is more suitable to detect defects in a gear system than the mean value. By using this statistical method, a cracked gear, as well a chipped gear can be identified, since both damages show a $10-50 \mathrm{~N}$ higher value of the contact force than a healthy gear set. However, the detection of a crack in a shaft is more challenging. The values of the cracked shaft and the healthy gear set are very close and the differences between them are vanishing small.

Note that the mean values of the different signals do not show any remarkable differences, which leads to the conclusion that calculating the mean of a signal is not suitable to detect a fault in a system. The only difference exist at a speed of $20 \mathrm{~Hz}$ where the mean of the chipped gear is much higher than the rest. However, this is an exception, which could be caused by a random noise appearance in the system, and does not necessarily reveal a chipped gear.

Note also that the values of a cracked shaft show only a small difference in comparison to the healthy gear set, in both the RMS and the mean plot. That means that an identification of a cracked shaft cannot be clearly made if statistical methods are applied to analyze the contact force in the time domain. 
A similar result can be obtained if the standard deviation of the raw signal of the individual faults are compared, which is shown in Figure 4.32. It can be seen, that the standard derivation of a cracked, as well as the chipped gear, has a higher value than the healthy gear set for each speed.

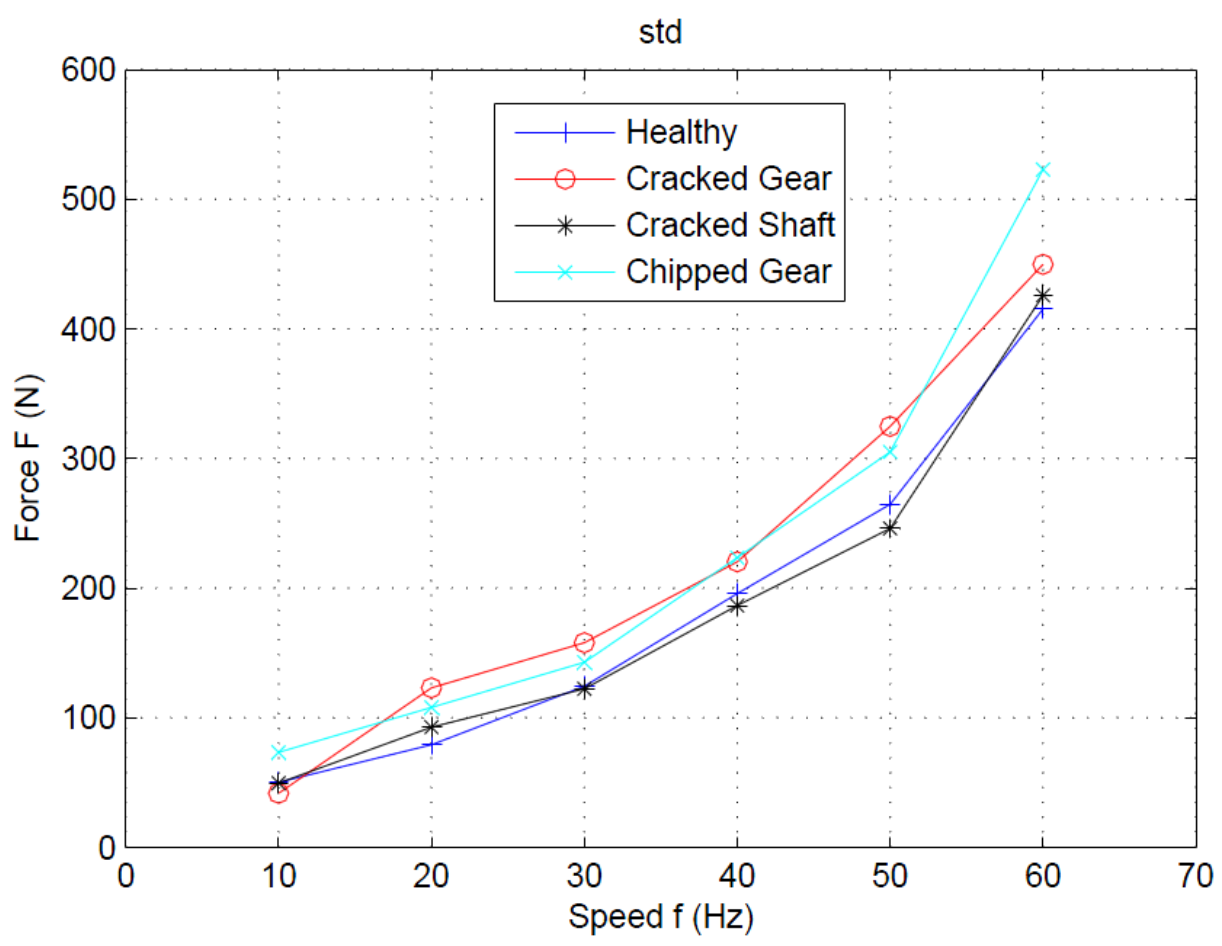

Figure 4.32. Standard deviation of different defects

Again, the values of the healthy system and the cracked shaft are close together and show only small differences. Therefore, a crack in the shaft cannot be identified, even if the standard derivation is taken into account. An interesting fact that can be seen in the above plot is that the standard derivation of all four graphs is increasing with the input speed. This is even the case for the last data point. While the mean value shows a low value of for this data point, the standard derivation shows a high value for this specific point. The increasing standard derivation with an increasing speed could be the cause of an increasing noise development in the system at higher speeds. 


\subsubsection{Analysis of the Signal in the Frequency Domain}

The result of the FFT analysis using statistical methods is presented in Figure 4.33 and Figure 4.34, where the mean values of the three different area blocks of all simulation setups are plotted. Again, the horizontal axis represents the input speed, while the vertical axis represents the magnitude of the contact force that appears in the FFT spectrum.
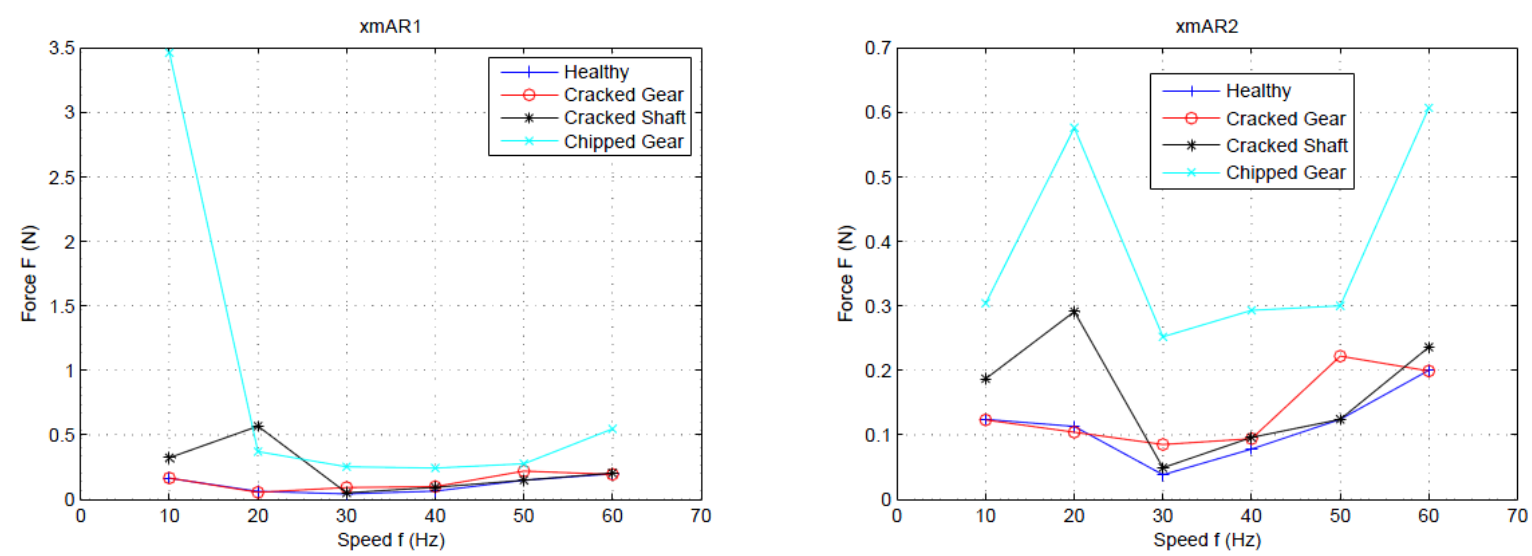

Figure 4.33. Mean values of area 1 and area 2

As can be seen, the chipped gear has the highest noise level, respectively the highest mean value of all simulation setups in all three area blocks. The only exception exist in plot for area 1, where the cracked shaft exceeds the value of the chipped gear. A clear identification of the chipped gear can easily be made by calculating the noise in the FFT.

On the other hand, this method shows some difficulties in detecting a cracked gear. Except for the area 2, the mean values of the cracked gear are close to the healthy gear, which means that the average value of the cracked gear is almost the same for the healthy gear. This can be explained due to the fact that a cracked gear creates 
only a slightly higher force and a slightly higher noise level in the FFT spectrum. Therefore, this analysis is not fully suitable to detect a cracked gear and a cracked shaft, but a chipped gear.
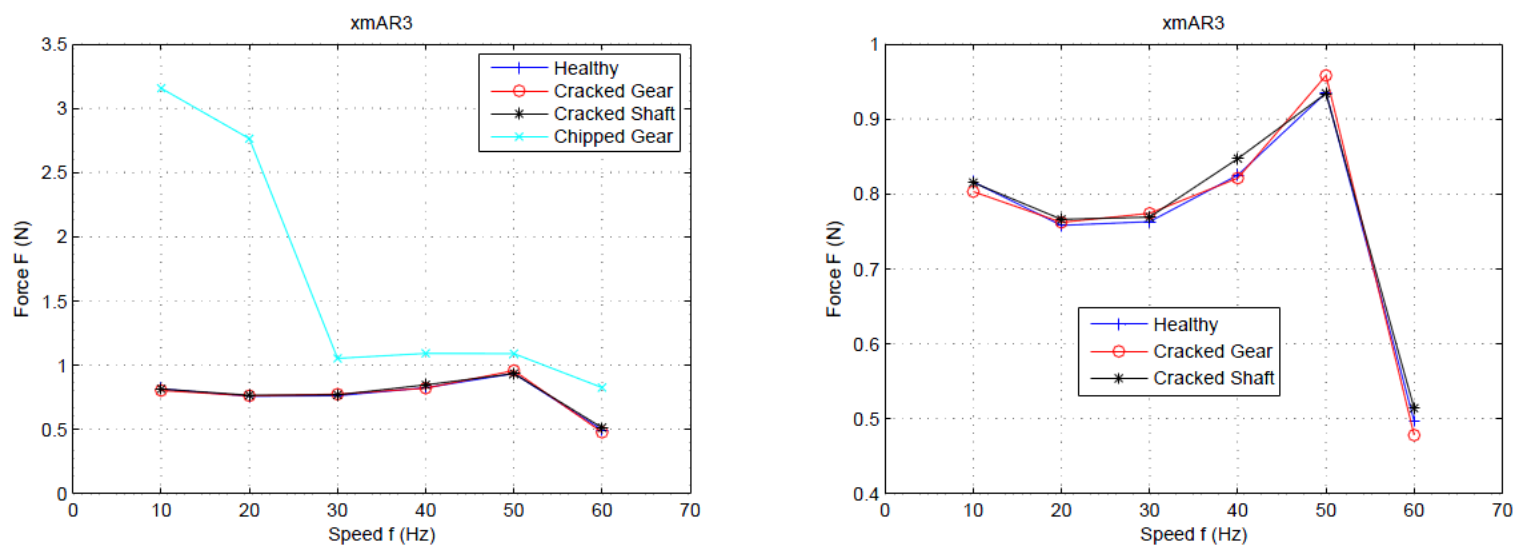

Figure 4.34. Mean values of area 3

Nevertheless, further research has to be made in order to get a clearer picture of the dependence between system faults and noise development in the frequency spectrum. Therefore, the standard derivation comes into consideration for the next analysis. Figure 4.35 and Figure 4.36 show the standard derivation of the noise level in all three different area blocks.

The plots reveal that the chipped gear shows the highest values of the standard deviation for all speeds. The only exception exist at a speed of $20 \mathrm{~Hz}$ in the area block 1, where the standard deviation of the cracked shaft is even higher than the chipped gear. This concludes that the standard derivation is an adequate method to detect major defects in a gear set, such as a chipped gear. 

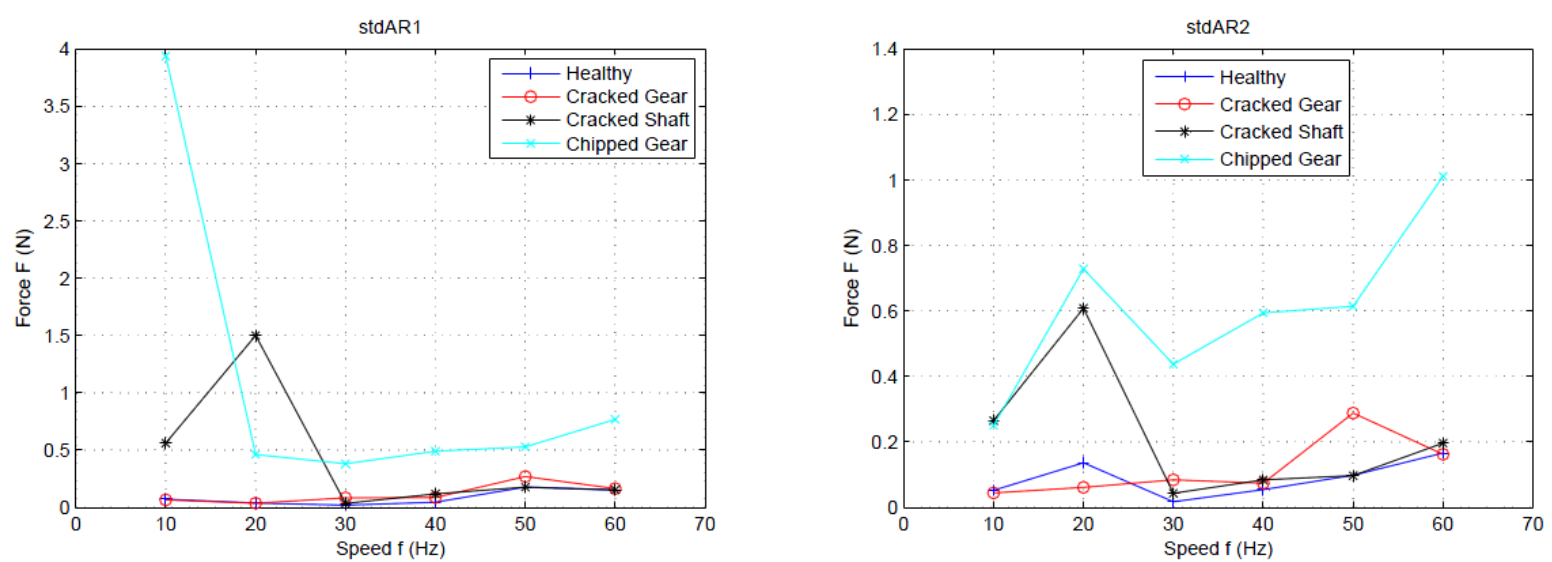

Figure 4.35. Standard deviation of area 1 and area 2 in the FFT plot

It is even possible to identify a cracked gear using the standard deviation to compare the noise profile in area 3. Although the differences between a cracked gear and a healthy set of gear are very small in area 1 and area 2, there are visible differences in area 3, where the standard deviation of the cracked gear has the second largest value. The differences are small but a cracked gear can be identified using this analysis.

Special interest arises the standard derivation of a cracked shaft at low speeds. Again, for area block 1 and 2 the values of the standard derivation exceed the values of the cracked gear, which leads to the conclusion that there might be a link between the contact force of a cracked shaft and the development of the noise profile at low speeds. However, further research needs to be made in order to fully reveal the appearance of random noise in a system. 

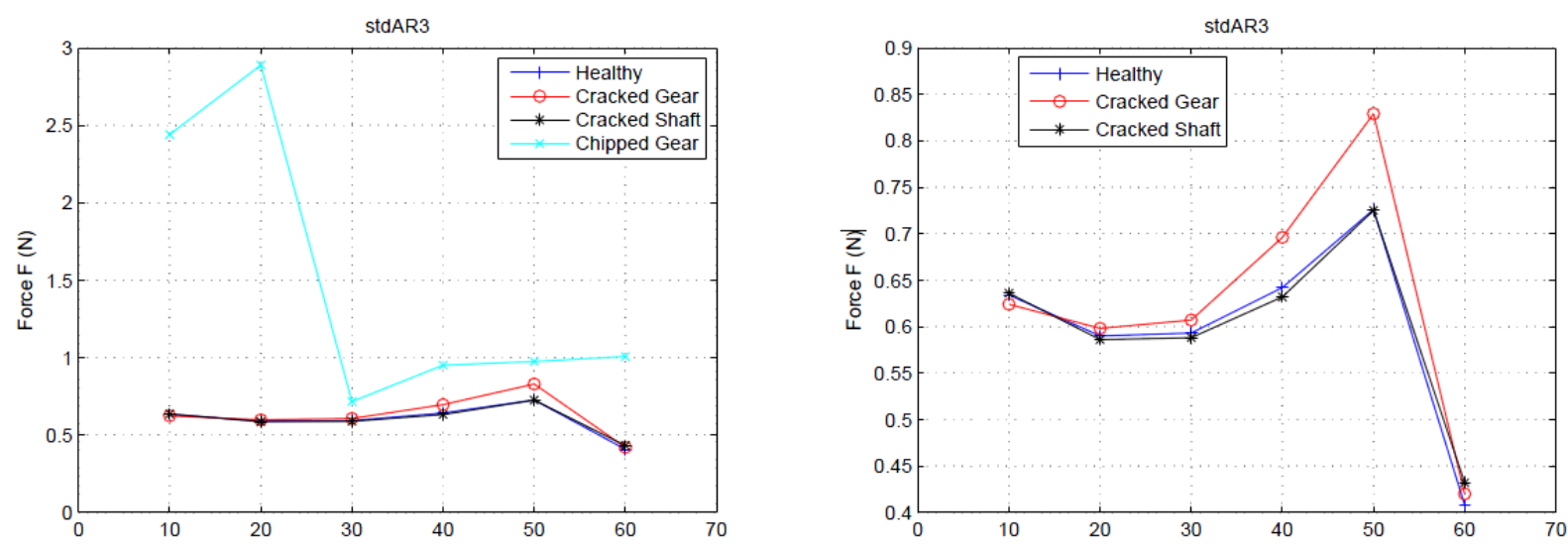

Figure 4.36. Standard deviation of area 3 in the FFT plot

The last methods is the comparison of the Sideband Energy Ratio (SER) between a healthy system, a cracked pinion, a cracked shaft and a chipped pinion. As previously mentioned, SER is calculated for the first three gear mesh frequencies. The result is presented Figure 4.37, where the SER values of the first two GMF's are plotted. Figure 4.38 shows the SER values for the third gear mesh frequency.
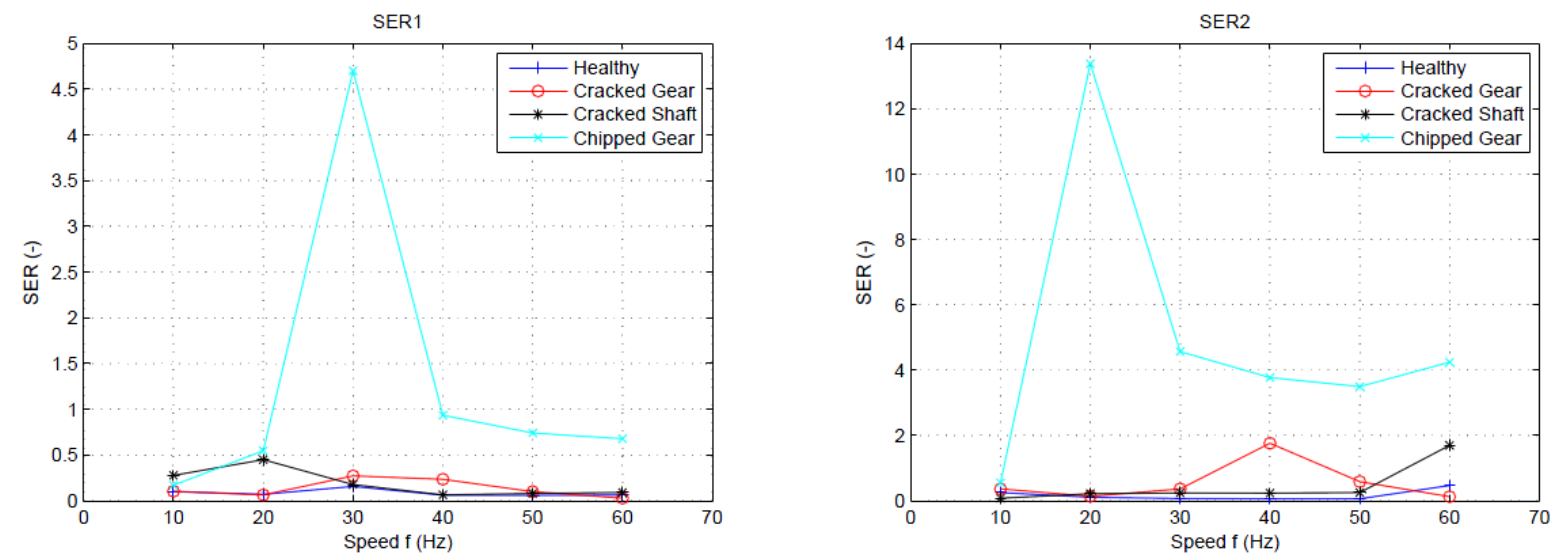

Figure 4.37. SER for the first two gear mesh frequencies

As can be seen, only the chipped gear can be identified as fault in the system when SER is used, since it is the only fault where SER is greater than one for all speeds. Although the SER values for a chipped gear at low speeds $(10 \mathrm{~Hz}$ and $20 \mathrm{~Hz})$ are below one in Figure 4.37, the SER is above one in Figure 4.38 for all speeds. There- 
fore, the threshold is exceeded in at least one case and the chipped gear can be identified using an algorithm. This leads to the conclusion that the method of the Side Band Energy Ratio works properly in order to identify major gear defects such as a broken tooth.

However, SER has some difficulties to detect minor defects in the system. As seen above, the SER values of the cracked gear and the cracked shaft are below one. The only exemption, where the cracked gear exceeds the SER value of one is at the second gear mesh frequency at a speed of $40 \mathrm{~Hz}$. SER does not detect a cracked shaft.

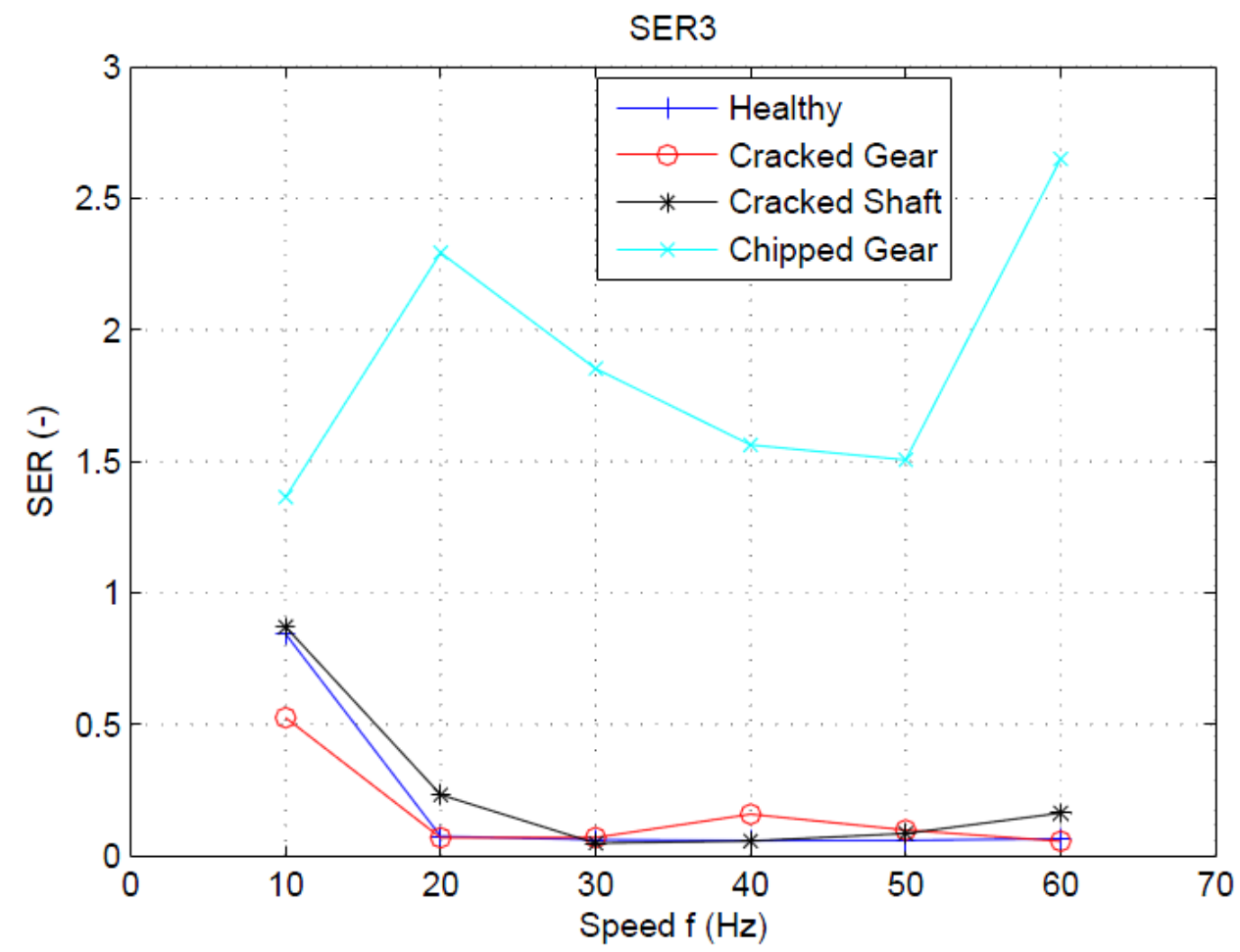

Figure 4.38. SER for the third gear mesh frequency 


\section{Summary and Conclusion}

It was shown that is possible to create the gyroscopic effect in MSC Adams, when a disk is mounted asymmetrical on a flexible shaft, which is supported with flexible bearings. The presence of the gyroscopic effect can be measured as angular displacements around the precession axis. It was shown that an increasing bearing stiffness has a small effect on the precession motions, since the bearings become more rigid and only the flexibility of the shaft accounts for gyroscopic effect. Furthermore, it was shown that the disk position on the shaft has a significant influence on the gyroscopic effect and vanishes when the disk is placed symmetrically onto the shaft. This result confirms the theory of a Jeffcott-Rotor. However, more simulations could be performed in order to do more research on the gyroscopic effect in rotor systems. Especially, different materials of the shaft could be used in order to analyze the influence of the precession motion. Furthermore, the simulation results could be verified with a simple practical shaft disk model in future.

The second analysis in this paper included the research on health detection methods. Several defects that included a cracked pinion, a cracked shaft and a chipped pinion were built-in in a simple fixed axis gear and the contact force between the gears was monitored and used to perform the FFT, 3D FFT and Wavelet analysis. The results of the 2D FFT show that that a chipped pinion and a cracked shaft can be visually identified in the plot. In particular, the presence of sidebands reveal a fault in the system. However, the presence of sidebands in the FFT for a cracked gear were too low in order to identify a minor defect. The same applies for the 3D FFT, where major differences between a chipped pinion and a healthy pinion exist. Minor differences were 
present when a cracked shaft, as well as the cracked pinion, were compared with a healthy gear set.

Furthermore, the Wavelet transform was used in order to analyze the vibration response of the system and to identify faults. It was demonstrated that Wavelet is a suitable method to detect a cracked shaft, as well as a chipped pinion. However, the differences for a cracked pinion and healthy gear set were small at a constant speed. Nevertheless, the Wavelet analysis detected a cracked gear when a linearly increasing speed is applied to the system, which confirms that Wavelet Transform is a powerful tool in health condition monitoring, especially for non-constant speed profiles. However, a reference to a healthy system is always needed in order to identify faults.

The last analysis included statistical methods on fault detection. The standard deviation, the root mean square, the mean and the Sideband Energy Ratio were used to compare the contact force in the time domain, as well as in the frequency domain. It was shown that the root mean square, as well as the standard deviation, is suitable to detect defects at the pinion. Under certain circumstances, the statistical methods can be applied to the FFT in order to detect a crack in the gear. Furthermore, it was shown that with the Sideband Energy Ratio detects only major defects in the system such as a chipped gear. 


\section{REFERENCES}

[1] Dutt, J. K., Nakra, B.C. (1995). Dynamics of Rotor system on Flexible Support with Gyroscopic Effects. Mechanics Research Communications, Vol. 22, No 6, pp.541-545.

[2] Wang, Z., Wahrburg, A, Rinderknecht, S. (2012). Consideration of Gyroscopic Effect in Fault Detection and Isolation for Unbalance Excited Rotor Systems. International Journal of Rotating Machinery, Volume 2012 (2012), Article ID 640794, 14 pages.

[3] Genta, G. (2005). Dynamics of Rotating Systems. Model with four degrees of freedom: Gyroscopic effect, Mechanical Engineering Series p 95. Springer. ISBN 978-0-387-28687-7.

[4] Federal Aviation Administration (1978). AERODYNAMICS OF FLIGHT. Basic Helicopter Handbook, p 7-18.

[5] Yoon, S.Y., et. al. (2013). Rotor Gyroscopic Effects. Control of Surge in Centrifugal Compressors by Active Magnetic Bearings. p 27-36. SpringerLondon. DOI 10.1007/978-1-4471-4240-9_2.

[6] Kraemer, E. (1993). Shaft with Non Central Disc. Dynamics of Rotor and Foundations, p 40-41. Springer. ISBN: 978-3-662-02800-1.

[7] He, M., et. al. (2005). Fundamentals of Fluid Film Journal Bearing Operation and Modeling. Proceedings of the thirty-fourth Turbomachinery Symposium, http://turbolab.tamu.edu/proc/turboproc/T34/t34-16.pdf.

[8] Adams, M. L. (2009). Campbell Diagrams. Rotating Machinery Vibration: From Analysis to Troubleshooting, Second Edition, p 163-165. CRC Press. ISBN 9781439807170.

[9] Friswell, M. I., et. al. (2010). More Complex Rotordynamics Models. Dynamics of Rotating Machines, p 439. Cambridge University Press. ISBN: 9780521850162.

[10] Genta, G. (2009). Forced vibrations of rotors: critical speeds. Vibration Dynamics and Control. p 584. Springer. ISBN: 978-0-387-79579-9.

[11] Brigham, E. O. (1998). The Fast Fourier Transform and Its Applications. Prentice-Hall Signal Processing Series: Advanced monographs. Prentice Hall. ISBN: 0133075052.

[12] Kruzick, S. (2013). Common Discrete Fourier Series [online]. Signals and Systems. Retrieved 6 Dec. 2014 from http://cnx.org/contents/56341977d03e-45fe-b78d-837970014a30/Common_Discrete_Fourier_Series. 
[13] Naveen, A. J. (2009). Understanding FFT Windows. APPLICATION NOTE ANO14. LDS-Group. Retrieved 7 Dec. 2014 from http://de.scribd.com/doc/17735864/FFT-Windows.

[14] Gao, R. X., Yan, R. (2011). Short-Time Fourier Transform. Wavelets. Theory and Applications for Manufacturing. p 21-25 Springer. ISBN 978-1-44191545-0.

[15] Polikar, R. THE FOURIER TRANSFORM AND THE SHORT TERM FOURIER TRANSFORM. THE WAVELET TUTORIAL PART 2. Rowan University. Retrieved 7 Dec. 2014 from http://users.rowan.edu/ polikar/WAVELETS/WTpart2.html.

[16] Wasilewski, P. Daubecchies 10 (db10). Wavelet Browser. PYWAVELETS. Retrieved 7 Dec. 2014 from http://wavelets.pybytes.com/wavelet/db10/.

[17] Mathworks. Wavelet Scalogram Using 1D Wavelet. Wavelet Toolbox. Retrieved 7 Dec. 2014 from http://www.mathworks.com/help/wavelet/examples/wavelet-scalogram-using-1d-wavelet-analysis.html.

[18] MEADinfo (2012). Gear Efficiency Compariosn Table. Comparison of Gear Efficiencies - Spur, Helical, Bevel, Worm, Hypoid, Cycloid. Retrieved 7 Dec. 2014 from http://www.meadinfo.org/2008/11/gear-efficiency-spur-helicalbevel-worm.html.

[19] Sait, A. S., Sharaf-Eldeen, Y. I. (2011) A Review of Gearbox Condition Monitoring Based on vibration Analysis Techniques Diagnostics and Prognostics. Rotating Machinery, Structural Health Monitoring, Shock and Vibration, Volume 5. Springer. DOI: 10.1007/978-1-4419-9428-8_25.

[20] Hanna, J., et. al. Detection of Wind Turbine Gear Tooth Defects Using Sideband Energy Ratio. Wind energy resource assessment and forecasting. Technical Paper. GE Energy. Retrieved 7 Dec. 2014 from http://www.ge$\mathrm{mcs}$.com/download/monitoring/SER-Technical-Paper.pdf.

[21] Sawatzky, R. P. D. (2014) Vibration Based Planetary Gear Analysis and Damage Detection. Master Thesis. California Polytechnic State University San Luis Obispo. p 70. 


\section{Appendices}

\section{A: 3D FFT Results (exponential speed)}
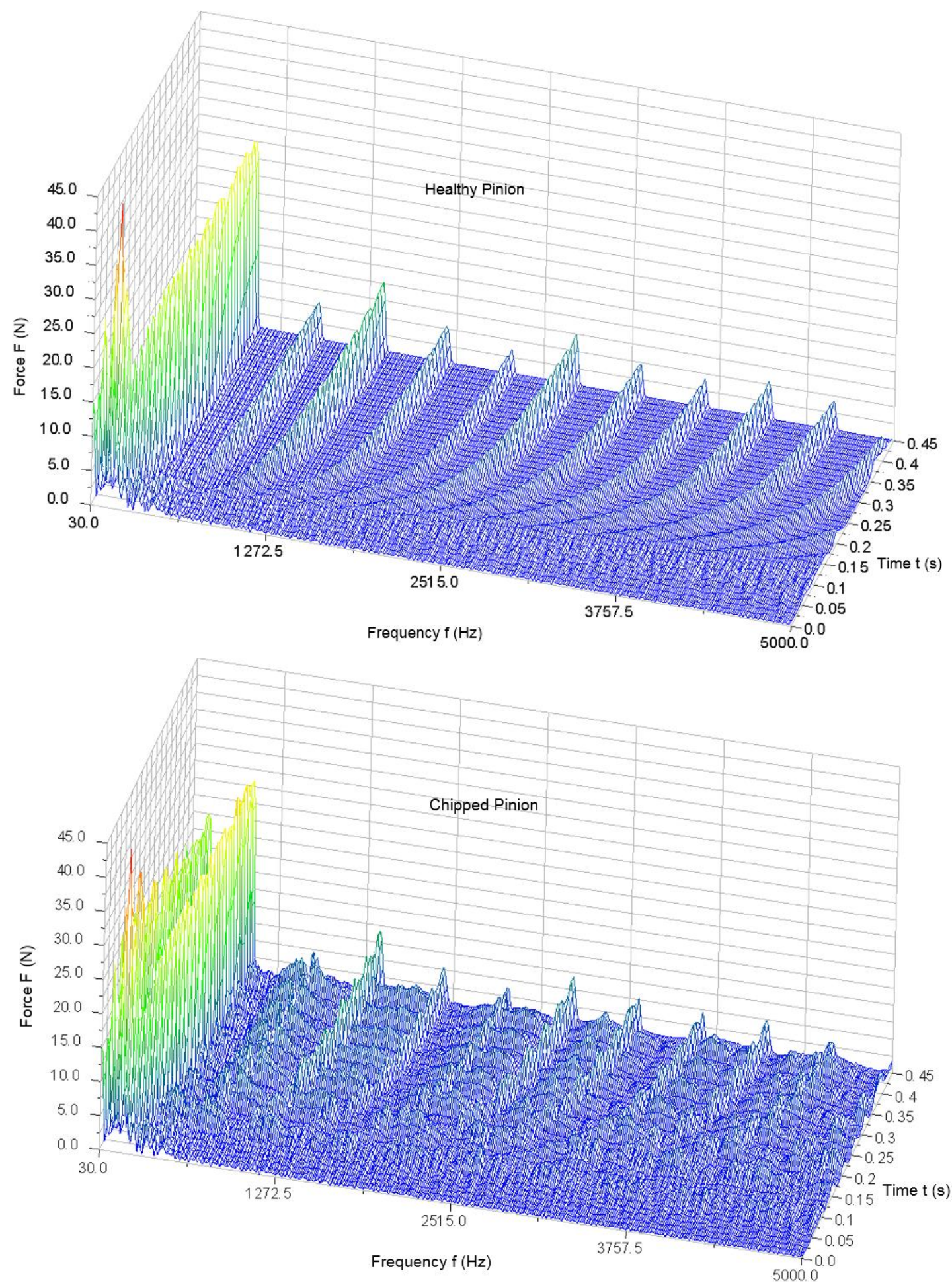

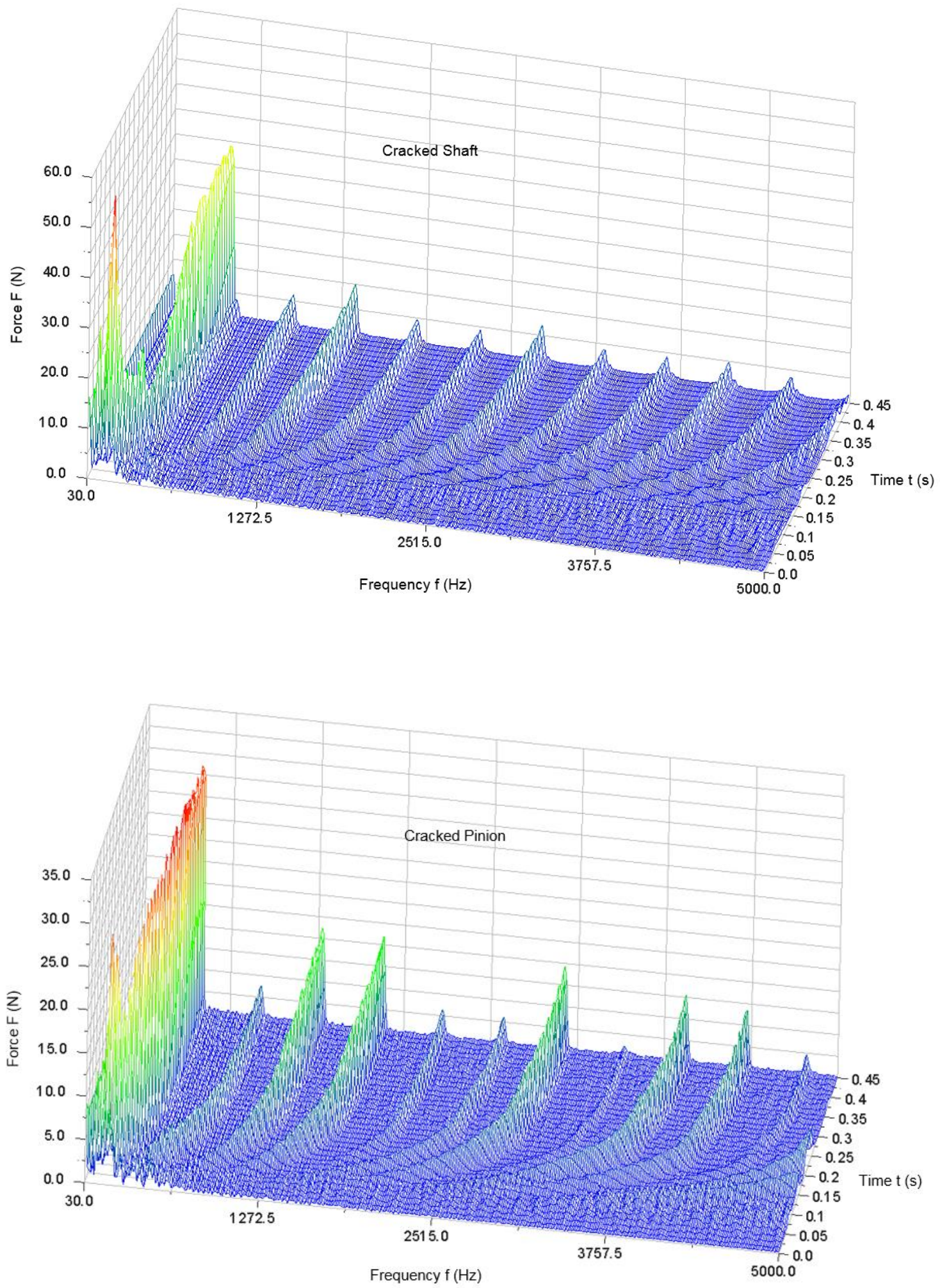


\section{B: Wavelet Toolbox Settings}

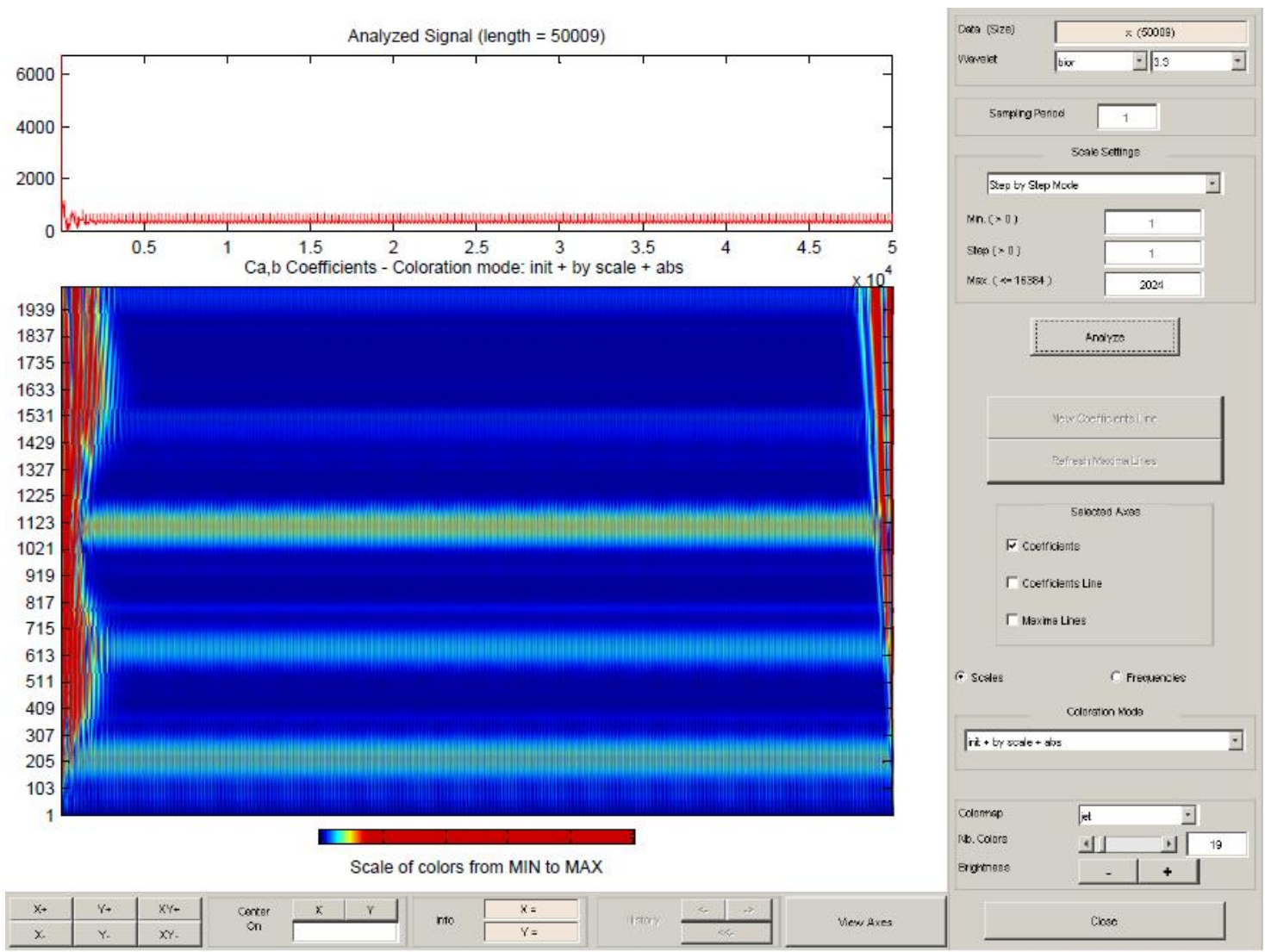




\section{C: Wavelet Linear Increasing Speed - Results}

Healthy Gear
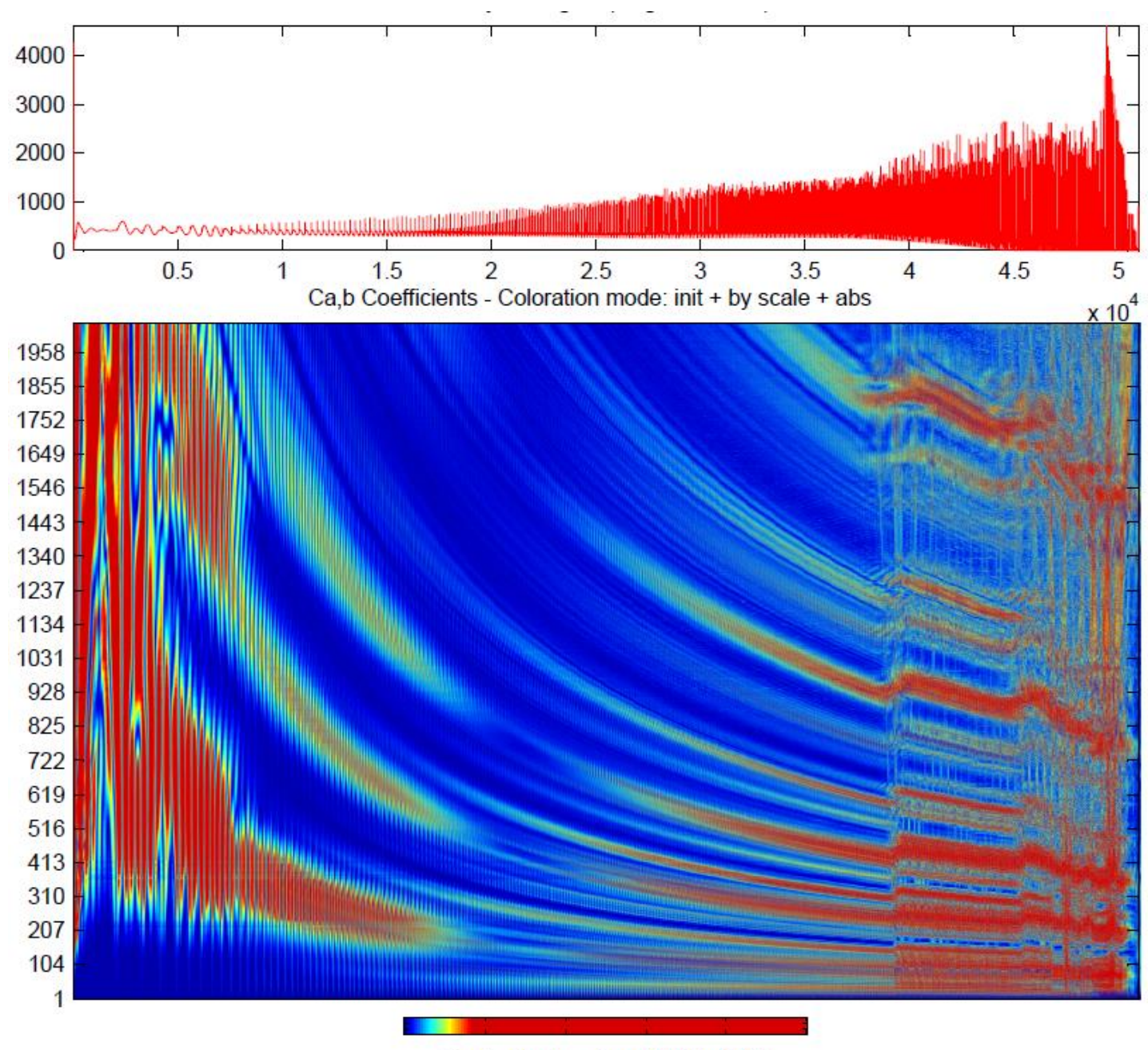

Scale of colors from MIN to MAX 


\section{Chipped Gear}
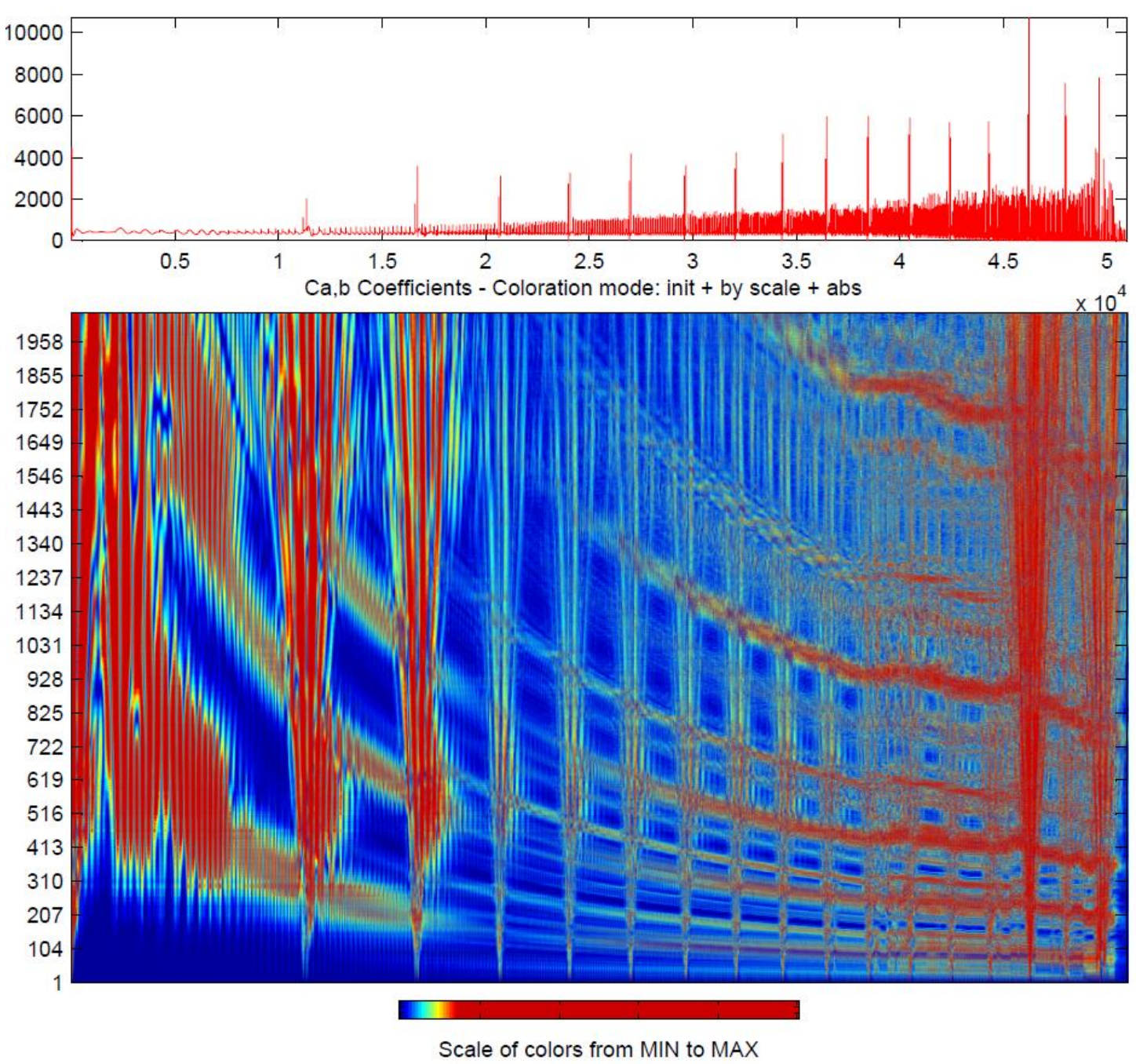


\section{Cracked shaft}
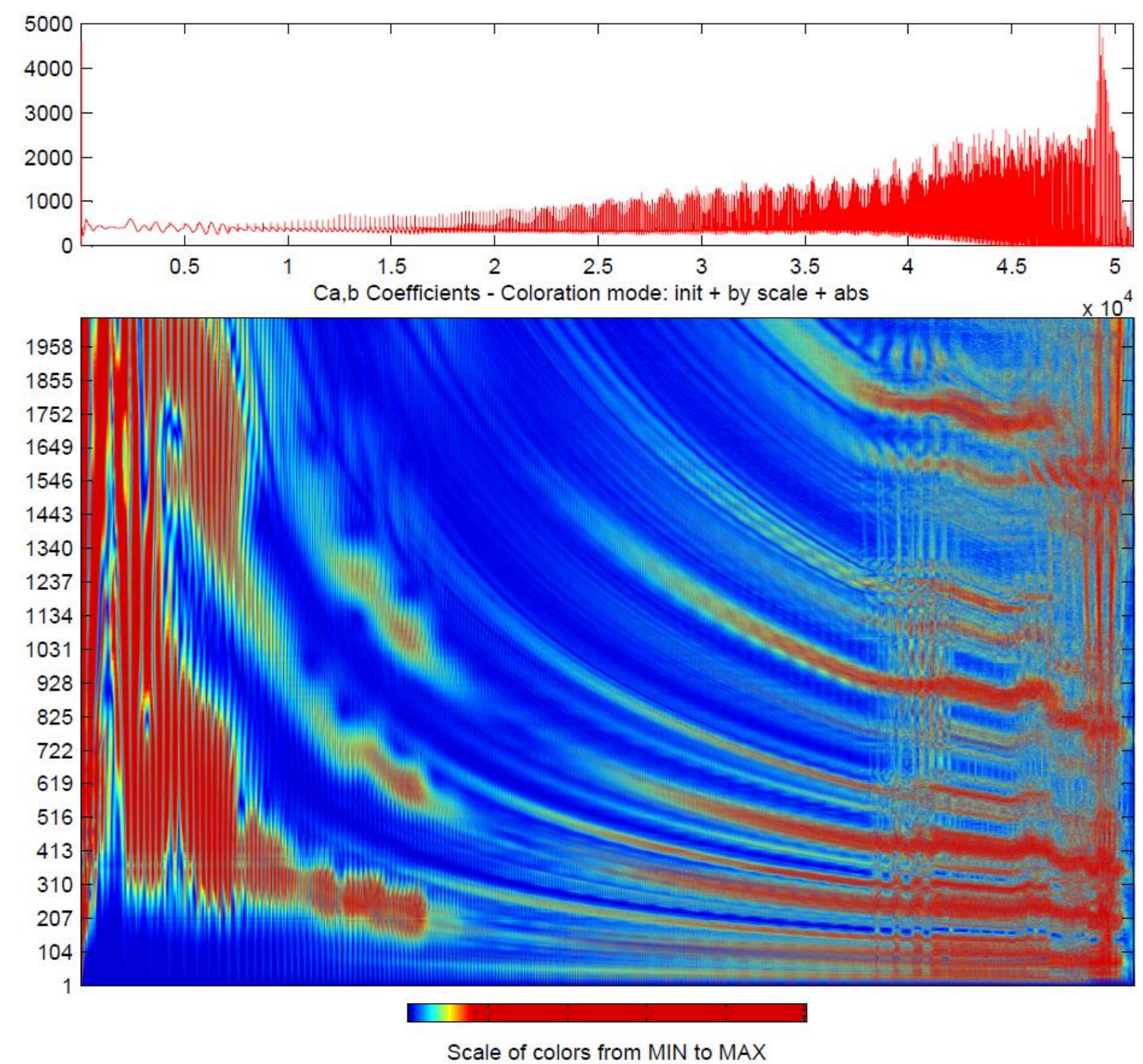


\section{Cracked Gear}
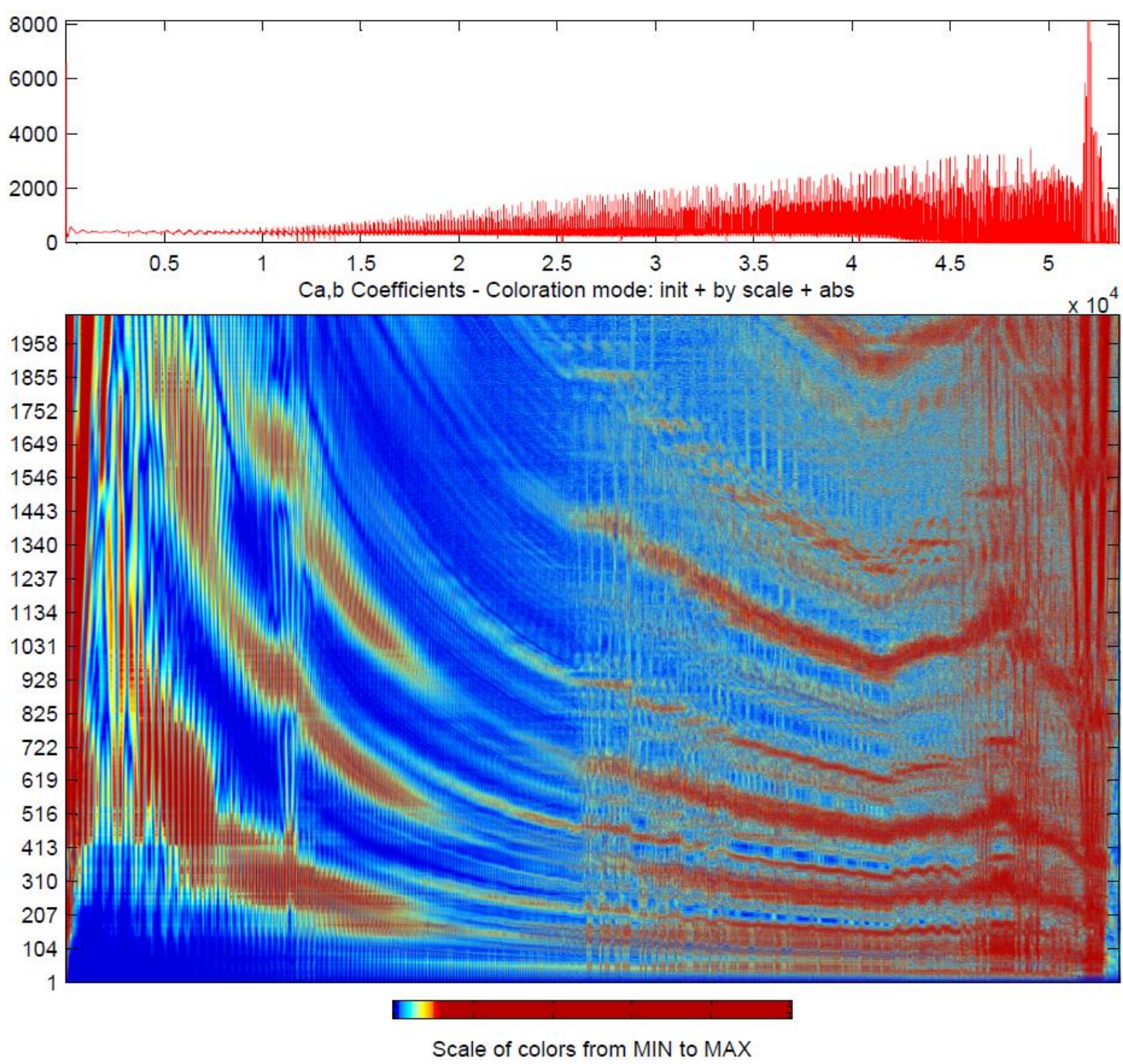


\section{D: How to Plot Wavelet from ADAMS Results}

1. Run "wavelet_simp" to import the data to matlab.

2. Choose "continuous Wavelet 1-D" in the appearing window.

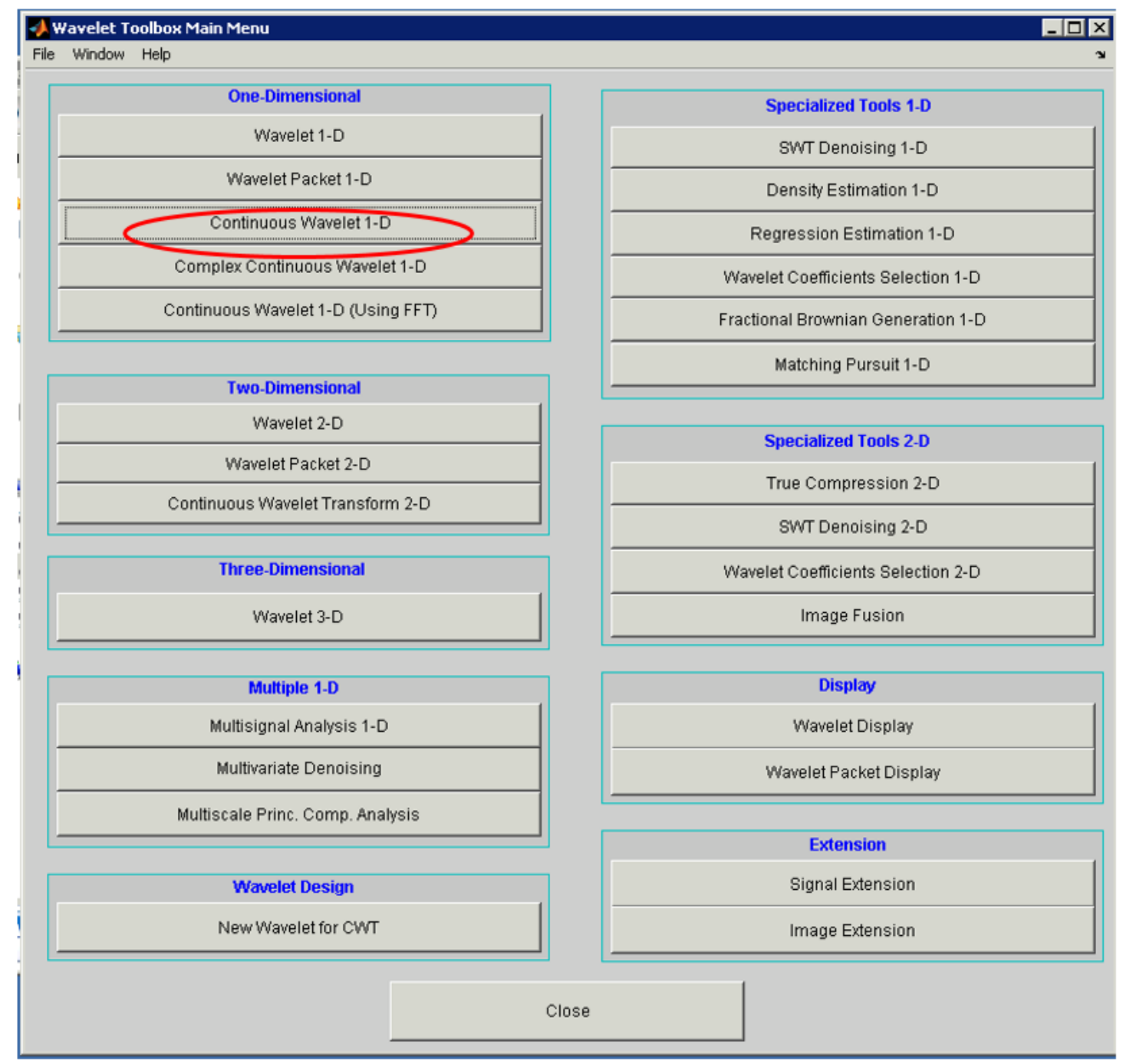

3. Then the "continuous Wavelet 1-D" window opens. Go to File -- $\rightarrow$ Import signal from Workspace and choose your data set from matlab Workspace.

4. Choose the following settings and click on analyse. 

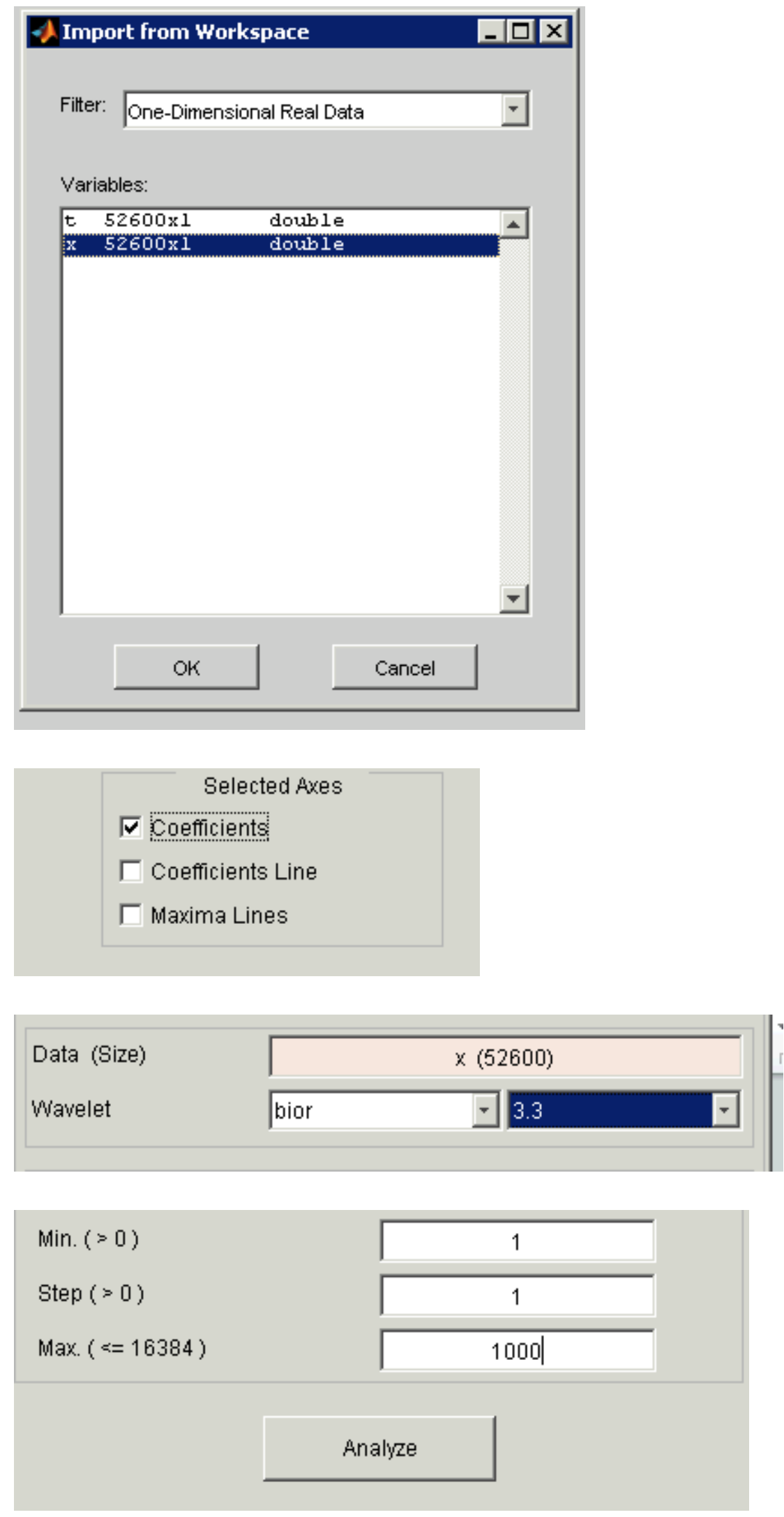

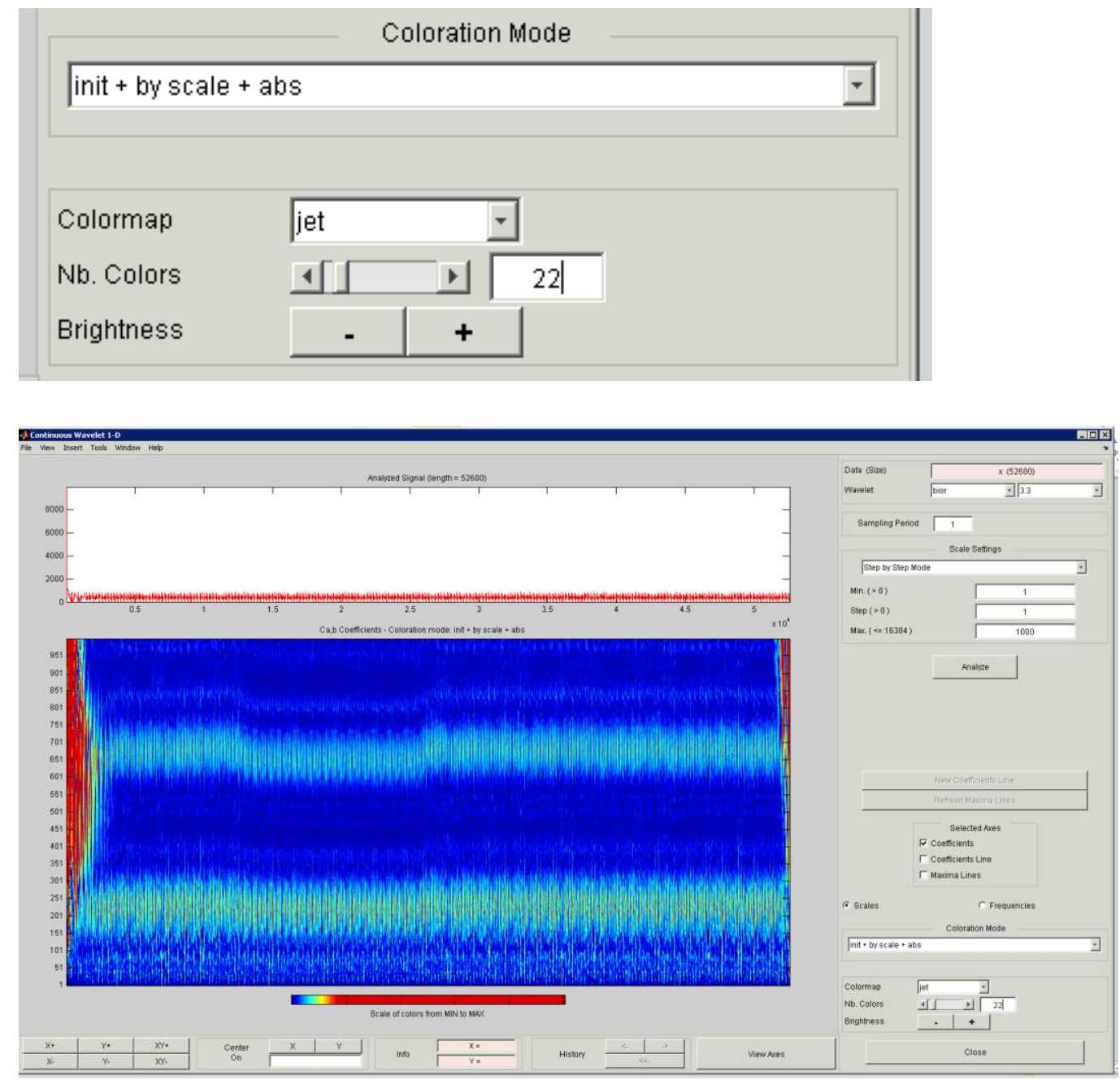


\section{E: Gyroscopic Effect Applied in Rotor Systems}

mnf file for the shaft is needed.

1. Open new ADAMS view file. Make sure that you choose the right units, which must be consistent to FEA model. Here the FEA model is created with SI units in ABAQUS. Therfore the same units must be chosen in Adams!

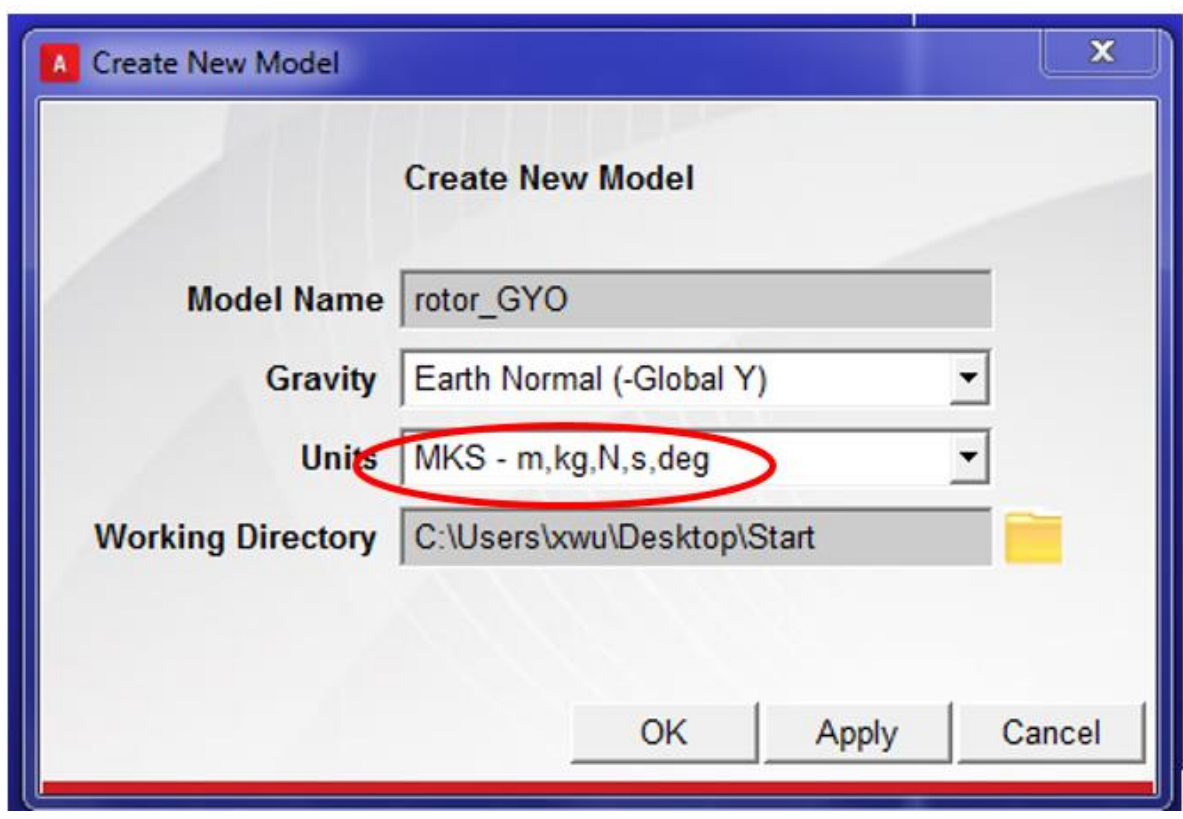

2. Import the *.mnf file first. Select

ADAMS/flex, create flex body through mnf import under body. 


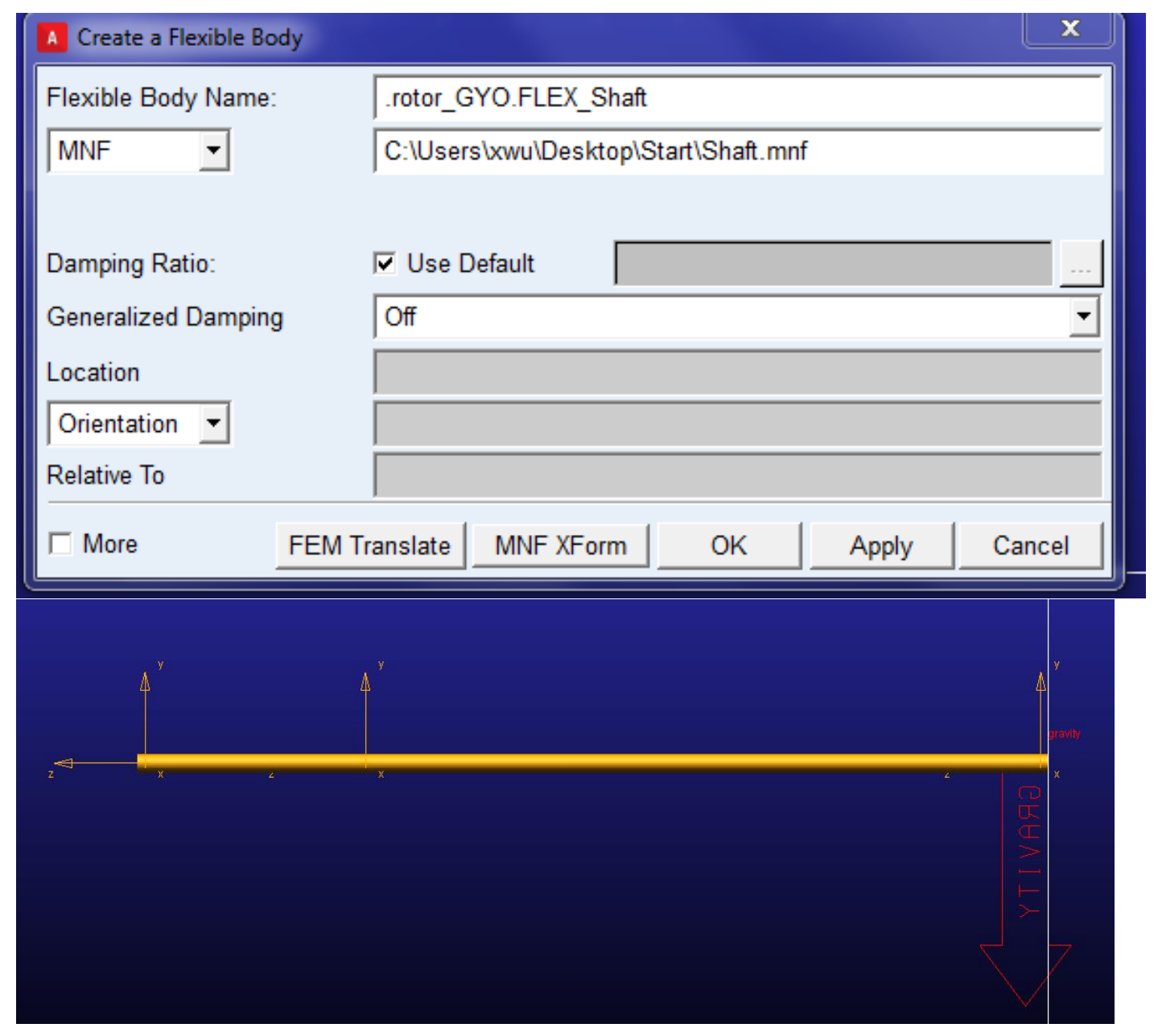

Notice the 3 local coordinates in the side view, which are generated by defining the multi points contraint in Abaqus.

3. Define dummy parts.

Select rigid, sphere under bodies.

\section{Geometry: Sphere}

\begin{tabular}{ll}
\hline New Part & $\square$ \\
$\sqrt{\checkmark}$ Radius & $(0.5 \mathrm{~mm})$
\end{tabular}

Rename it as DummyR. Repeat the process for another two dummy parts. 


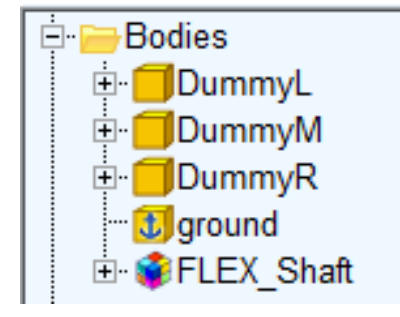

4. Connect the Dummy parts to the shaft

Use the fixed joints to connect the Dummy Parts with the flexible shaft (Repeat 3 times) to the nodes (appearing as INT_1000000 etc.) in the browser manager.

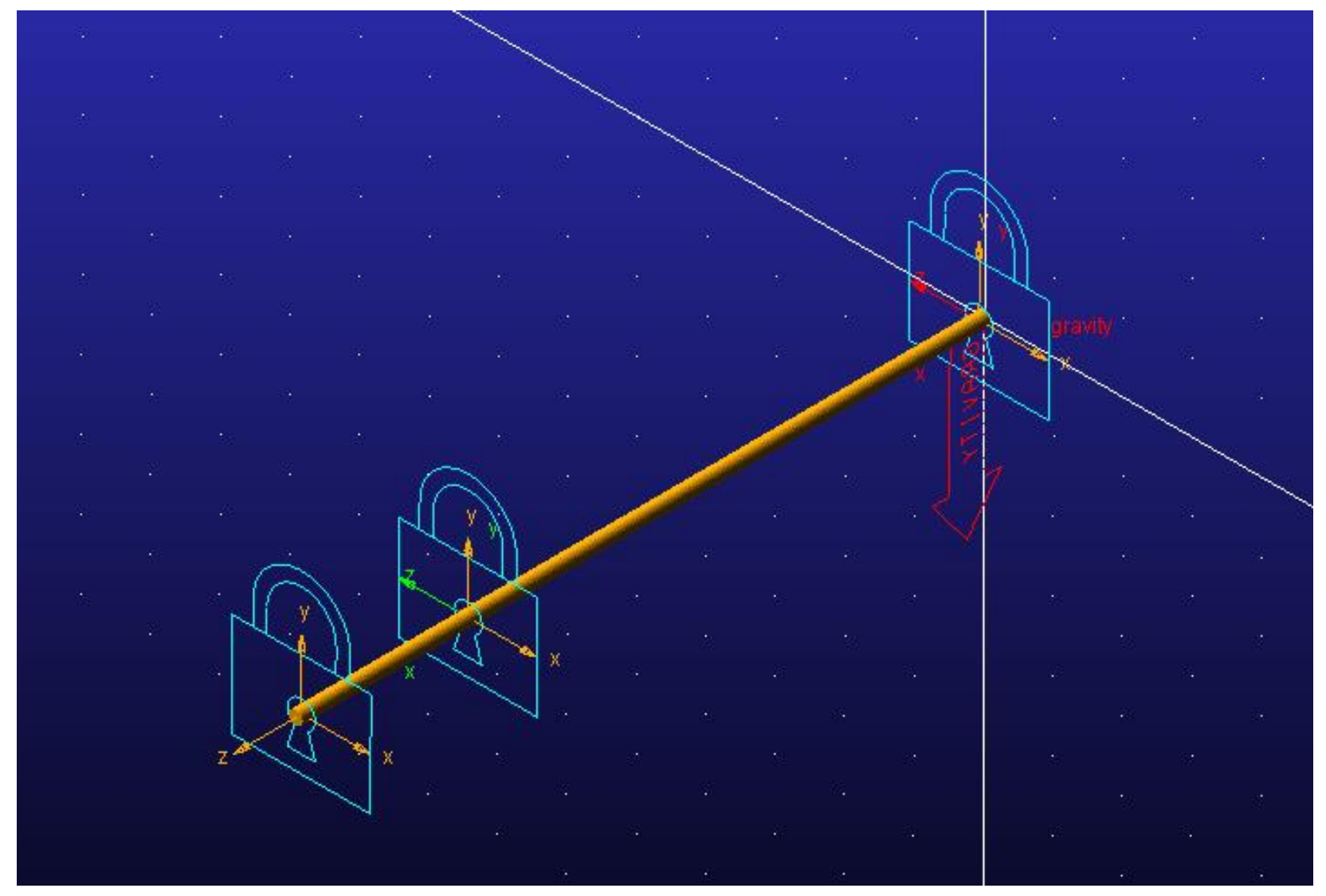

5. Import the bushing CAD model as the "Part Name" 


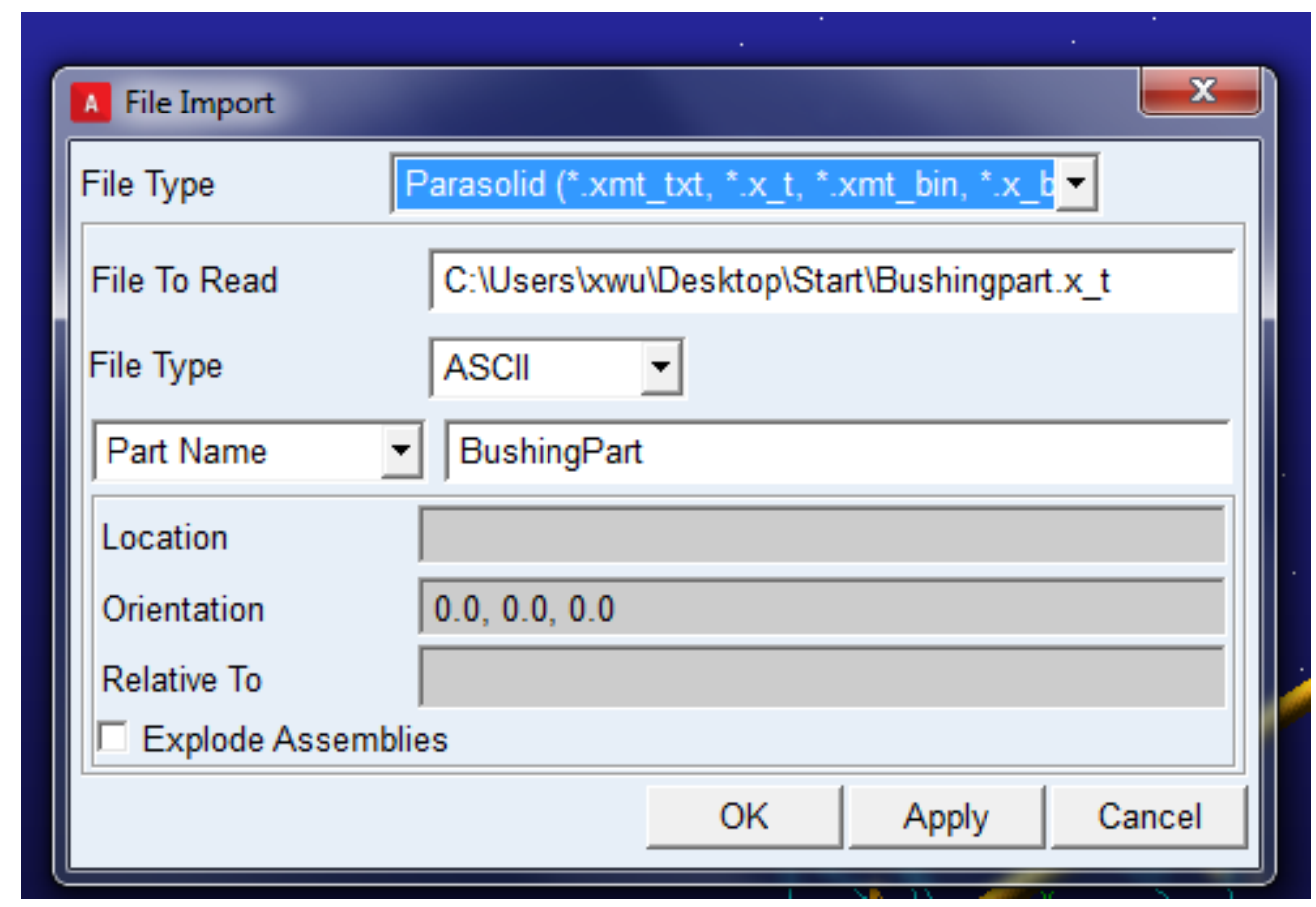

Then, the bushing part will be automatically placed to the origin of the coordinate system and the model.

\section{Assign the material properties to BushingPart}

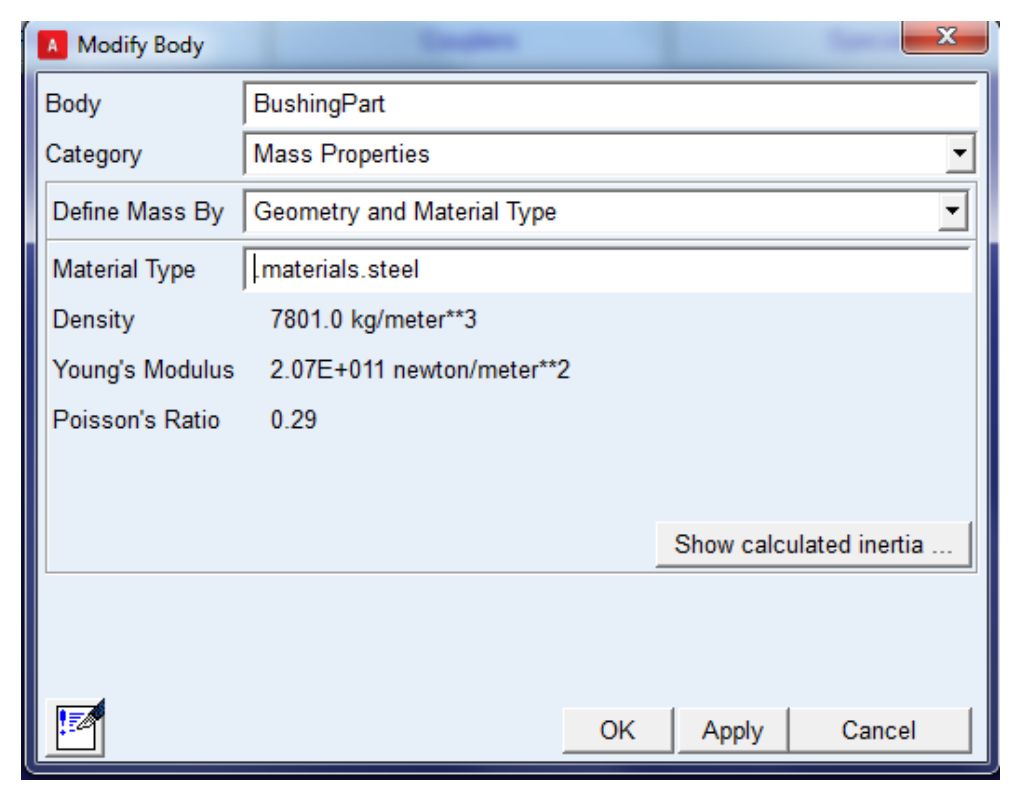

7. move $\stackrel{\lambda}{\square}$ and copy the BushingPart to the left to create the $2^{\text {nd }}$ Bushing. This is an easy way to create new identical bushing. 


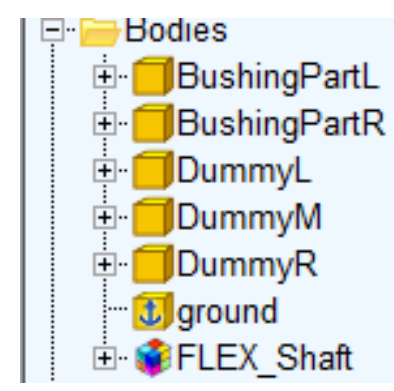

8. Fix those two bushings to ground. Select the center of mass of each bushing as the fix location.

9. Import the disk from CAD model (don't specify the location). It will locate at the origin of GCS.

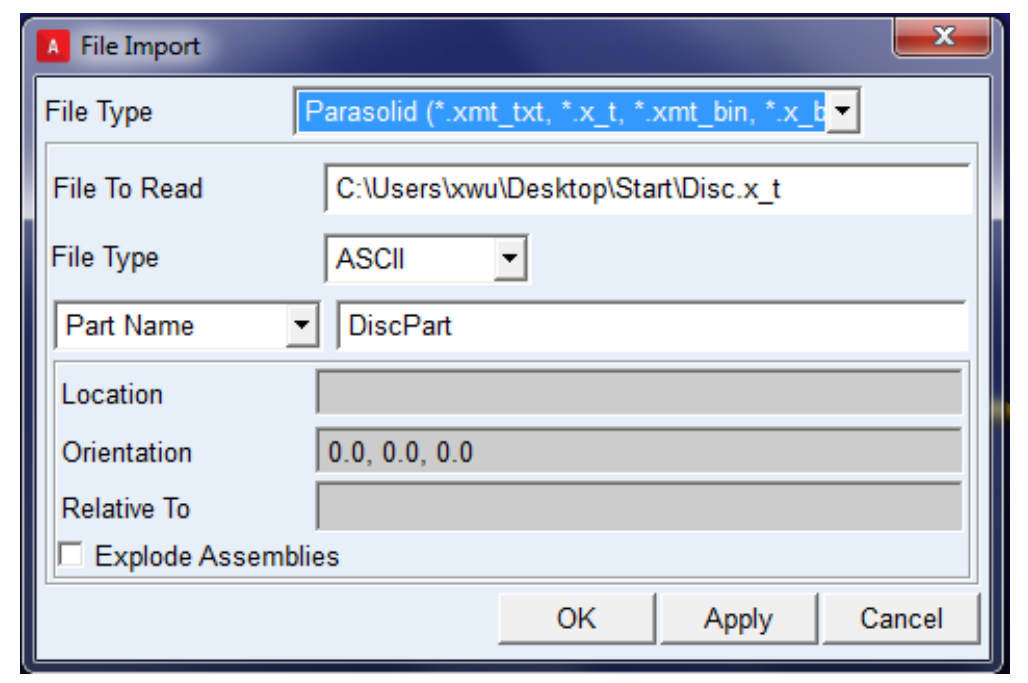

10. Assign the material "steel" property to it. (Here: steel)

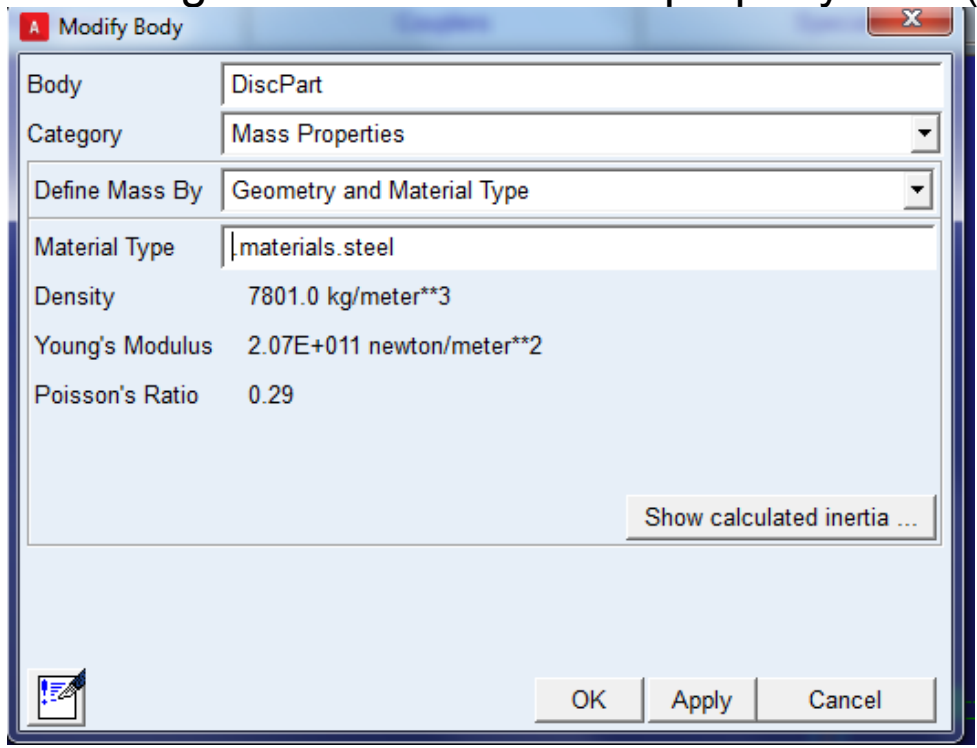


11. Place the disk to the disired location. Choose the .cm of the disk part as the point to move from.

12. Fix the disk to the dummy part. Select the $\mathrm{cm}$ of the dummyM as the location.

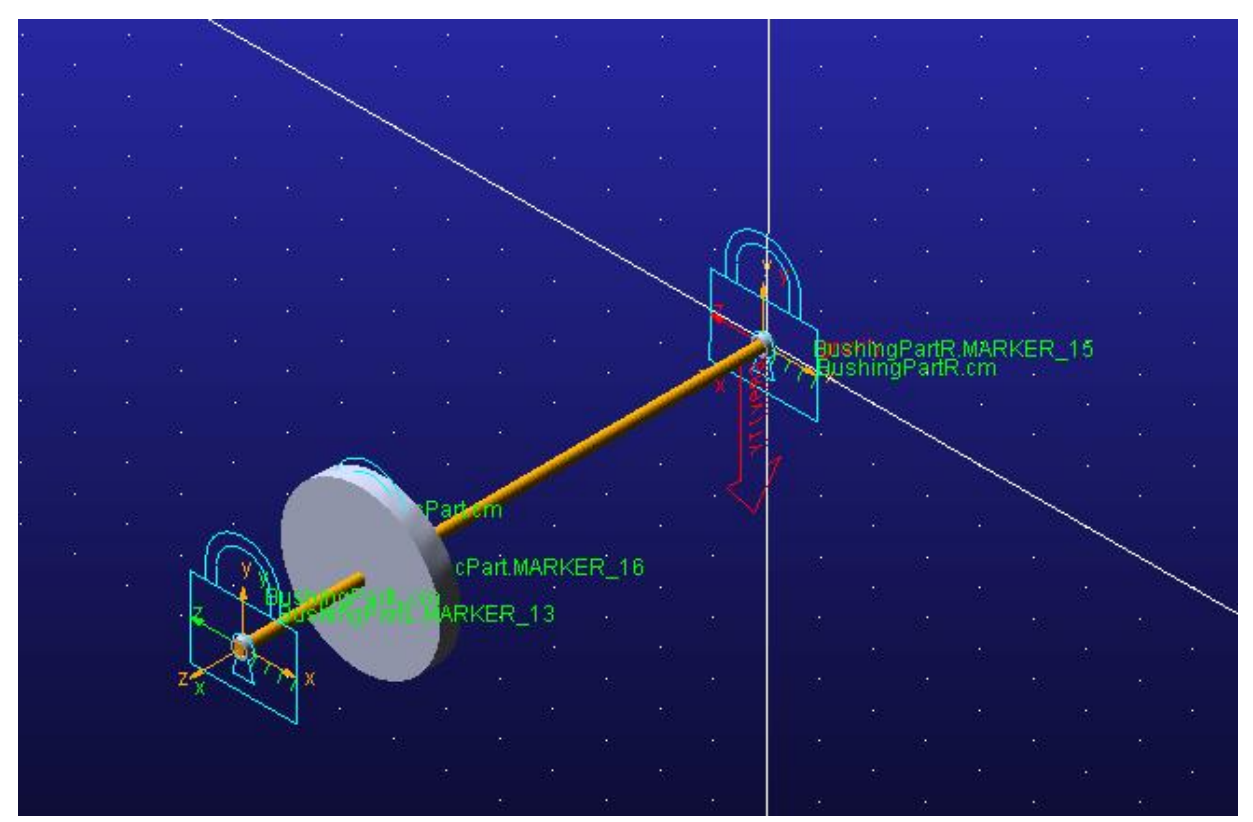

13. Apply actual bushings at those two locations, which will connect the BushingPart to its corresponding Dummy points. The location must be the center of the Dummy part or the constraint node at the flexible shaft.

14. Assign the parameters of the bushings. Change the stiffness to $1.0 \mathrm{e} 7 \mathrm{~N} / \mathrm{m}$. 


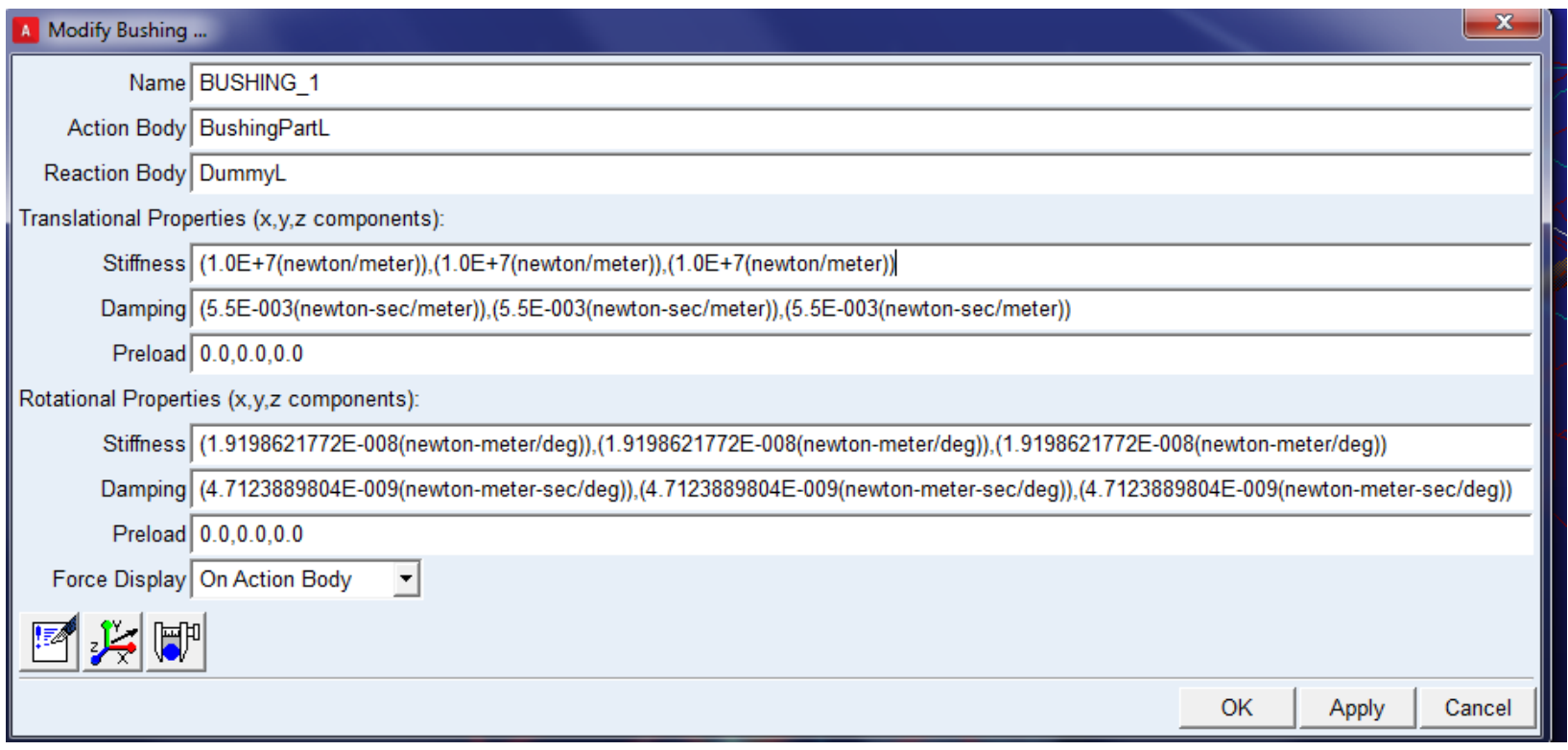

15. Important, this step is necessary to plot the Euler angles.

Create two markers at the disk location. The first one is placed at the ground the center of the flexible shaft node (where the disk is located) and rename it as "GroundMarker".

The second one is added to the disk part. The location is the same (flexible shaft node). Rename it as Diskmarker.

Marker
Add to Part
Orientation
Global XY Plane

16. Create a state variable defined by differential equations der elements.

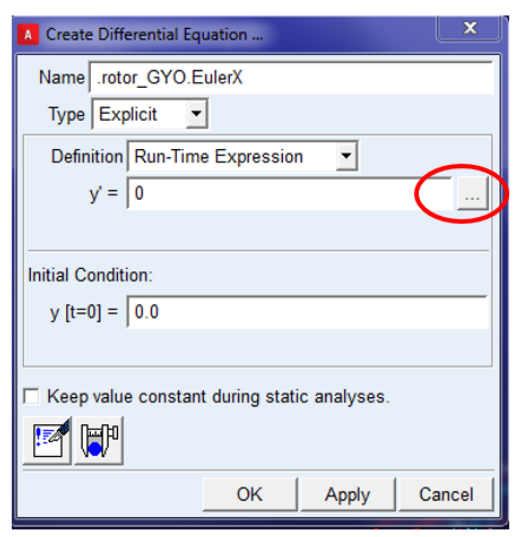


Another window will appear:

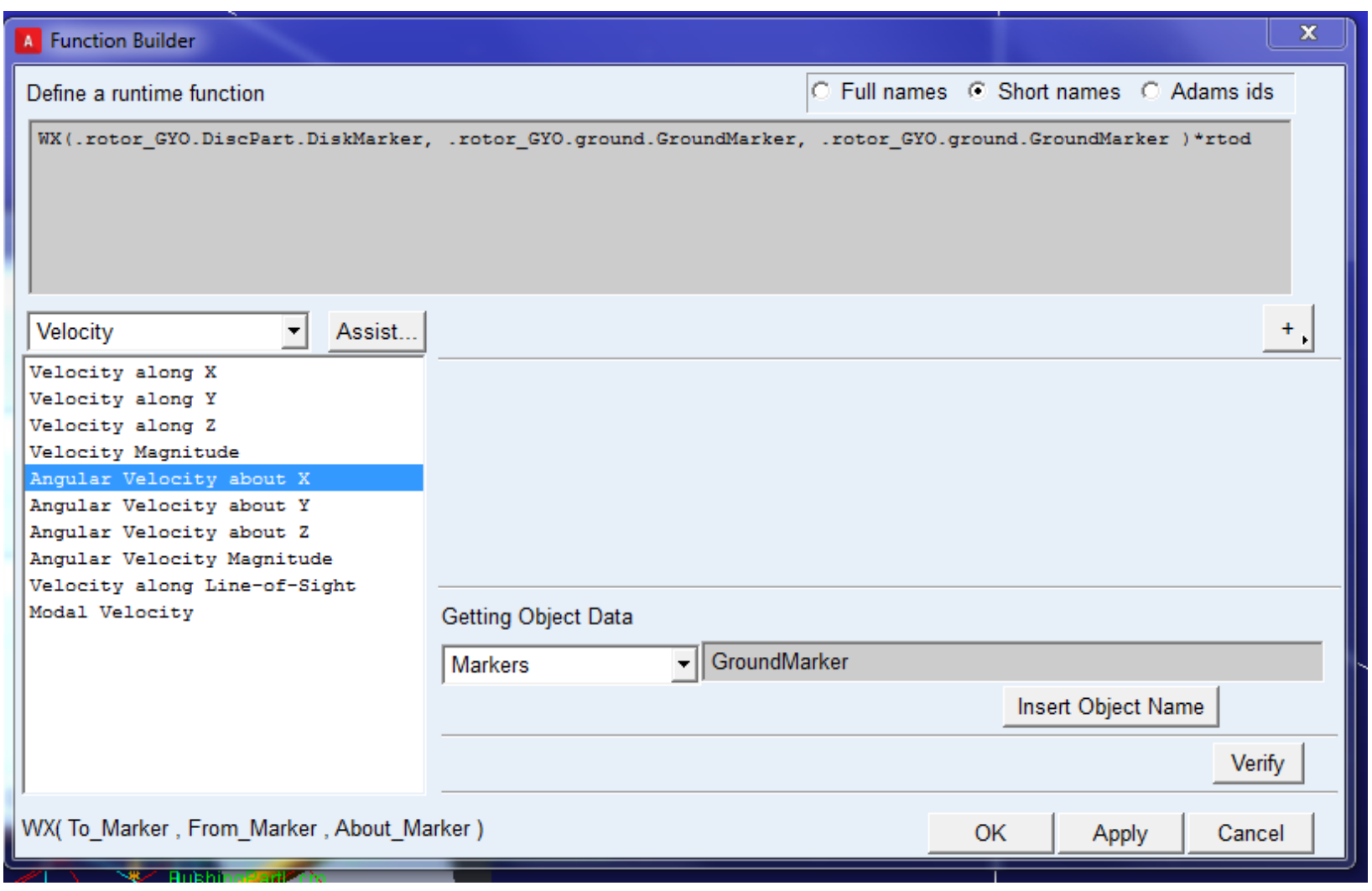

Right click the black at the right -side of Markers and find the desired marker. Double click "Insert Object Name", all of them will show in the function.

Type in "*rtod" to convert radians to degrees.

17. Create another 2 Eulaer angles by coping EulerX twice under Elements.

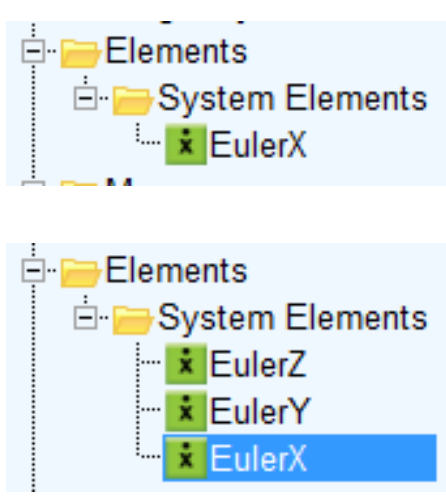




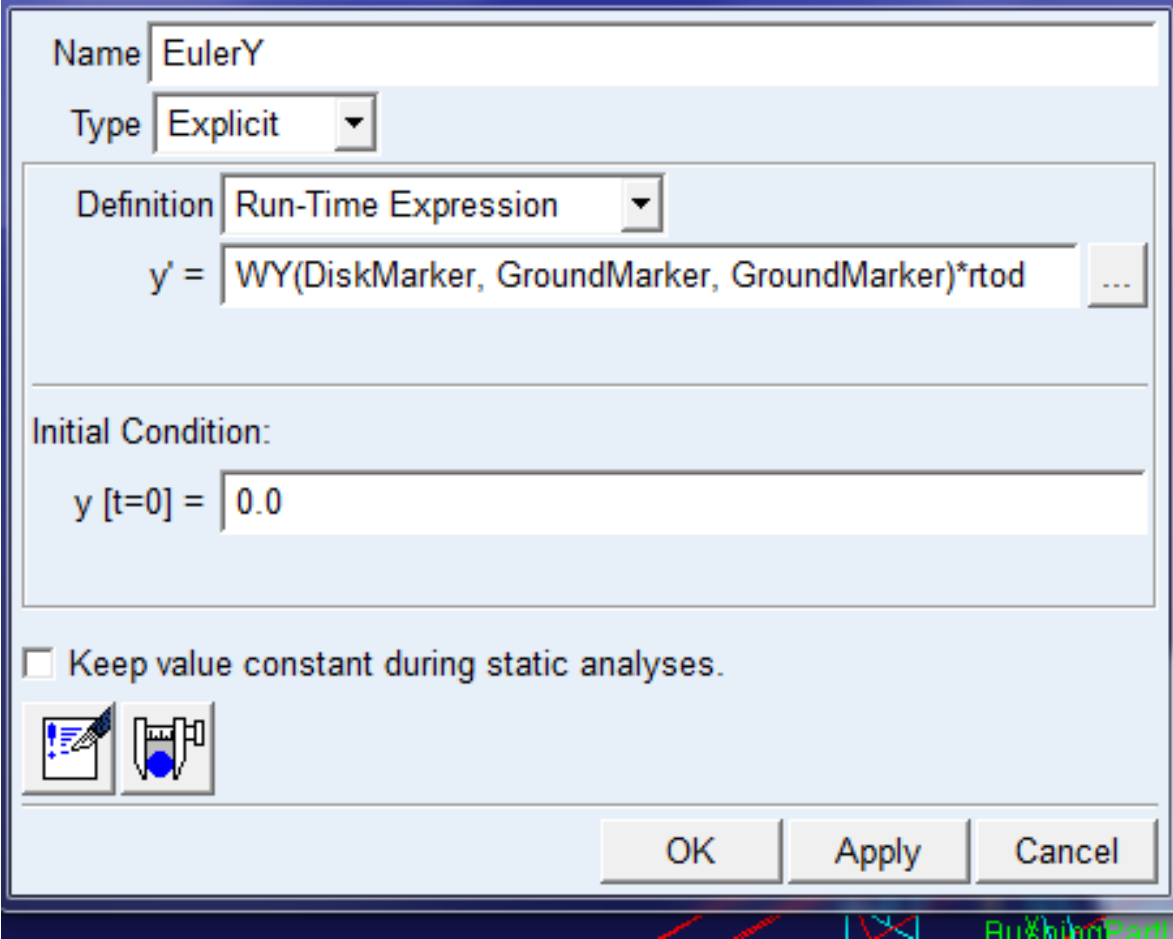

A Modify Differential Equation ...

Name EulerZ

Type Explicit ․

Definition Run-Time Expression

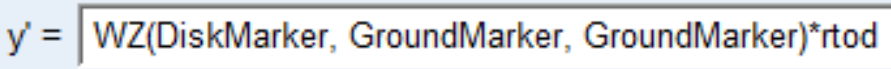

Initial Condition:

$$
\mathrm{y}[\mathrm{t}=0]=0.0
$$

ГKeep value constant during static analyses.

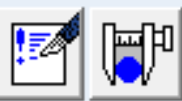

OK Apply 1 Cancel

Double click EulerY and Change WX to WY and WZ, respectively click OK once you finish. 
18. Apply the rotational speed to the dummy part attached to the right side of the shaft. Change to side view to apply the motion

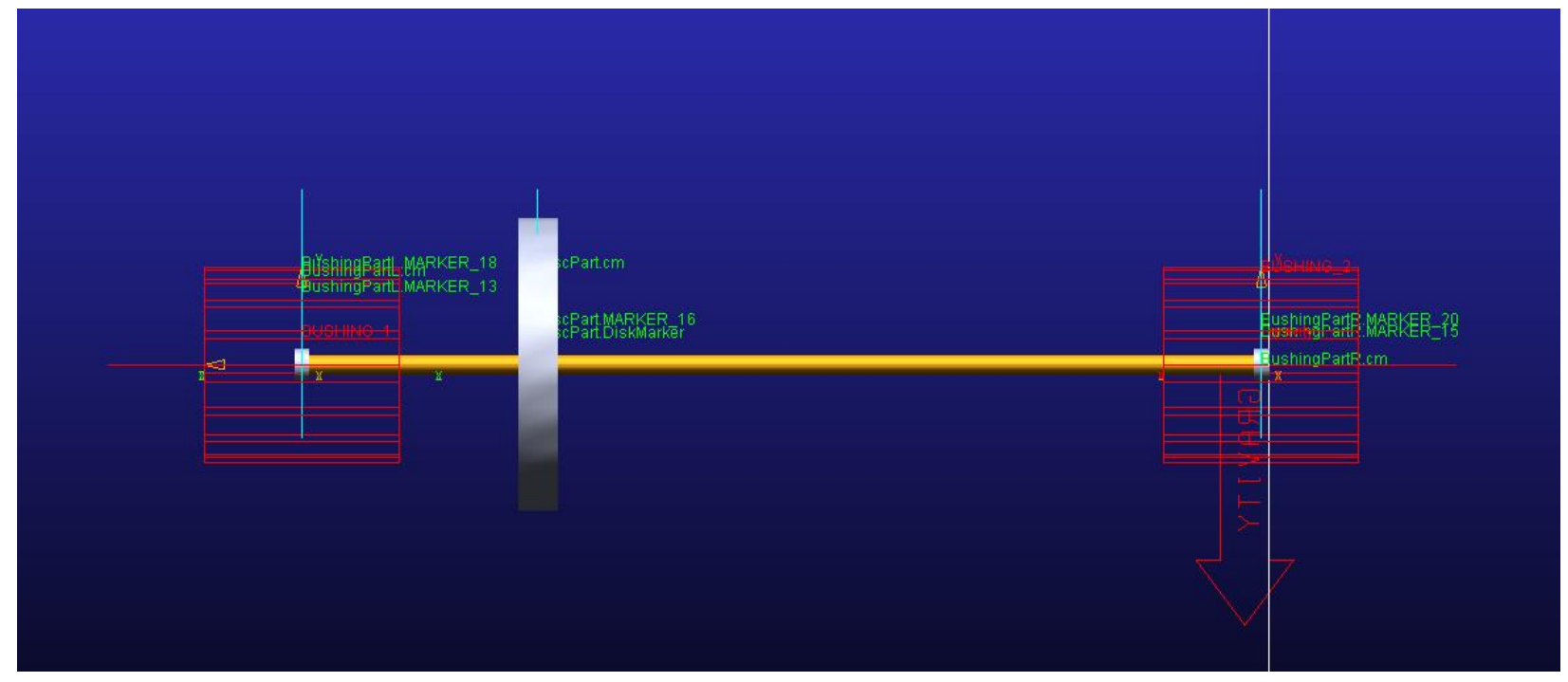

Since there is not revolute joint, you need to apply the motion from point motion

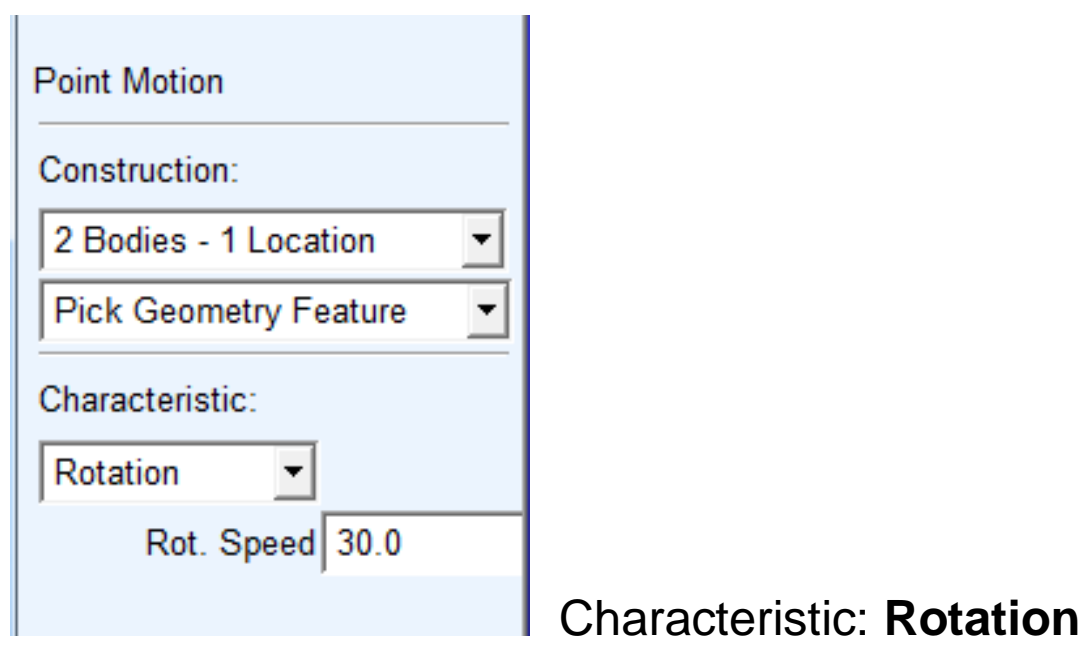

Choose DummyR, ground and DummyR.cm. The feature direction must be around the shaft (Important). In this case z-dirction.

19. Do the simulation

20. Plot Euler angles in post processing, go to the result set, plot EulerX $Y$ and $Z$. 


\section{F: How to define a motor torque curve in Adams}

1. Create a torque curve in ADAMS. Go to Elements $-\rightarrow$ spline

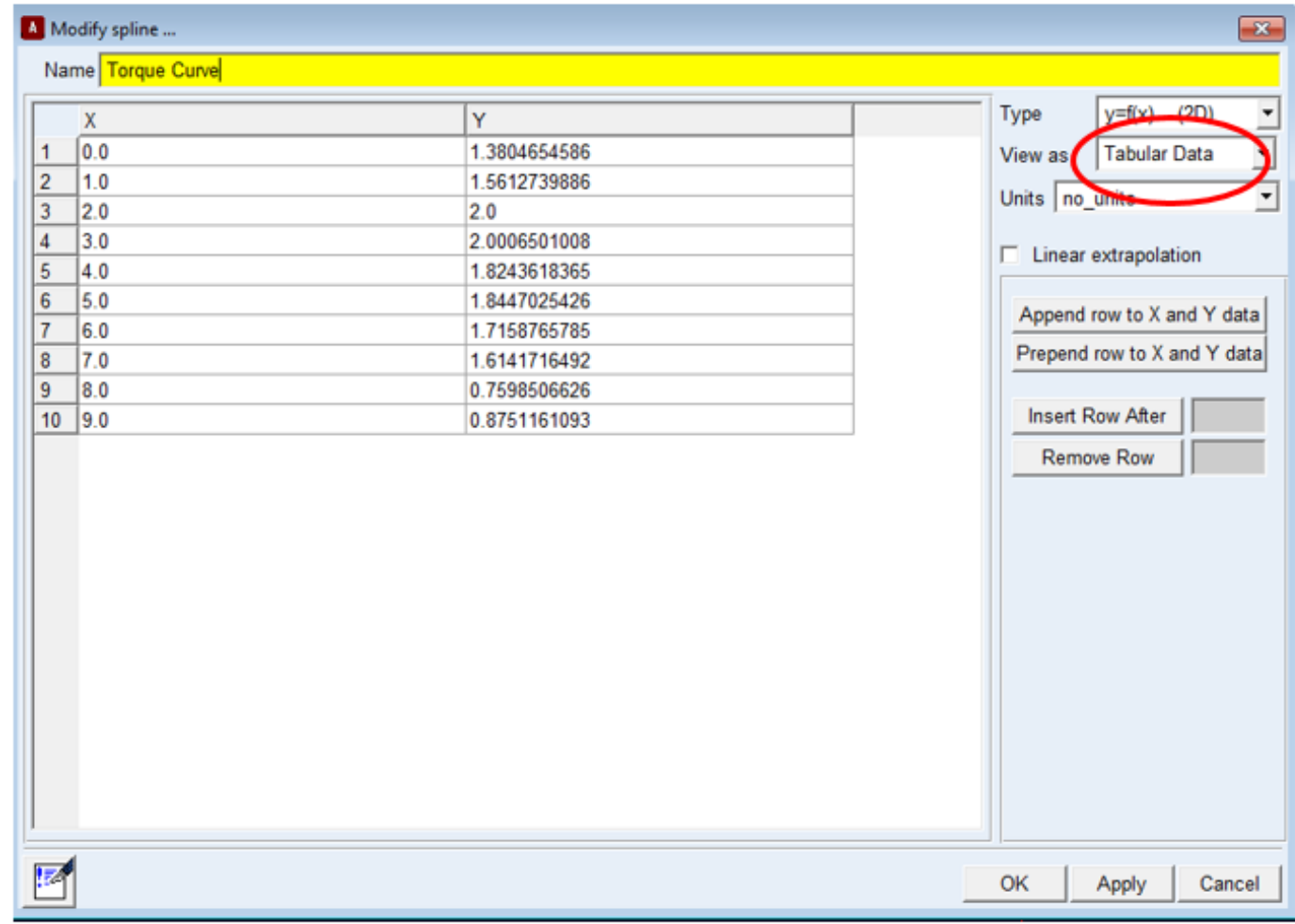

2. Create a system Variable from elements.

\section{Elements}

A Modify State Variable ...

Name VARIABLE_1

F(time,

Definition Run-Time Expression

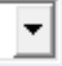

$\sqrt{\checkmark}$ Guess for $F(t=0)=\quad 0.0$

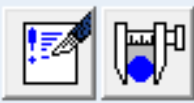

\begin{tabular}{l|l|l|} 
OK & Apply & Cancel \\
\hline
\end{tabular}


Input any speed you want. Here is $5 \mathrm{rad} / \mathrm{sec}$. VARIABLE_1 shows up under System

\section{Elements}

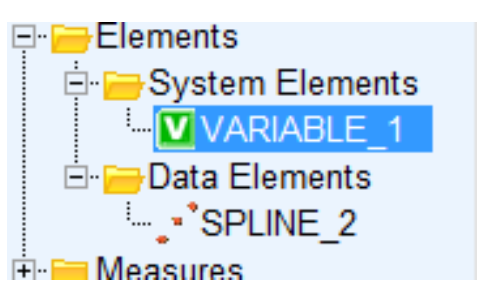

Note: The spline in this example is: SPLINE_2. You may have a different name.

3. Relate the variable to the motion.

Modify the motion. Delete the original speed and input the systems' variable name: VARVAL(VARIABLE_1), click OK
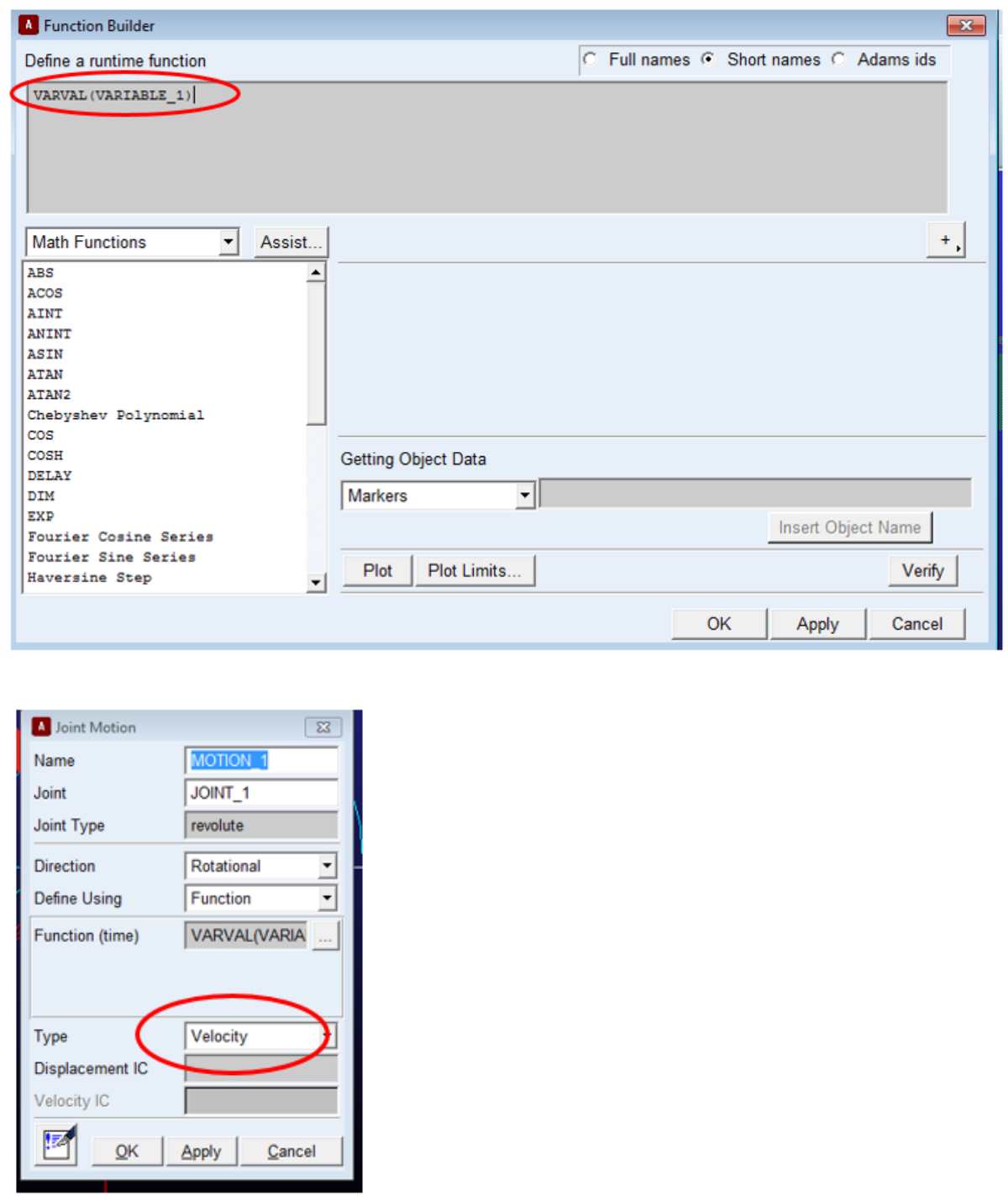
4. Apply a torque on the input link and relate it to the defined torque curve.

(1). Apply a torque on the input link and rename it as $\mathrm{Wu}$ _T (arbitrary)

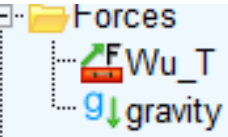

(2). Modify the applied torque Wu_T

\begin{tabular}{|c|c|c|}
\hline A Modify Torque & & $\underline{x}$ \\
\hline Name & Wu_T & \\
\hline Direction & On One Body, Fixed In Space & $\nabla$ \\
\hline Body & LinkOA & \\
\hline Define Using & Function & 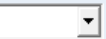 \\
\hline Function & AKISPL(VARVAL(VARIABLE_1),0,SPLINE_2) & $\ldots$ \\
\hline Solver ID & 1 & \\
\hline Torque Display & On & $\nabla$ \\
\hline PED & Apply & Cancel \\
\hline
\end{tabular}

Choose "All functions" and "Akima Fitting Method" in Function Builder; Delete the original speed and input the following command:

AKISPL(VARVAL(VARIABLE_1),0,SPLINE_2).

Use your own spline name here.

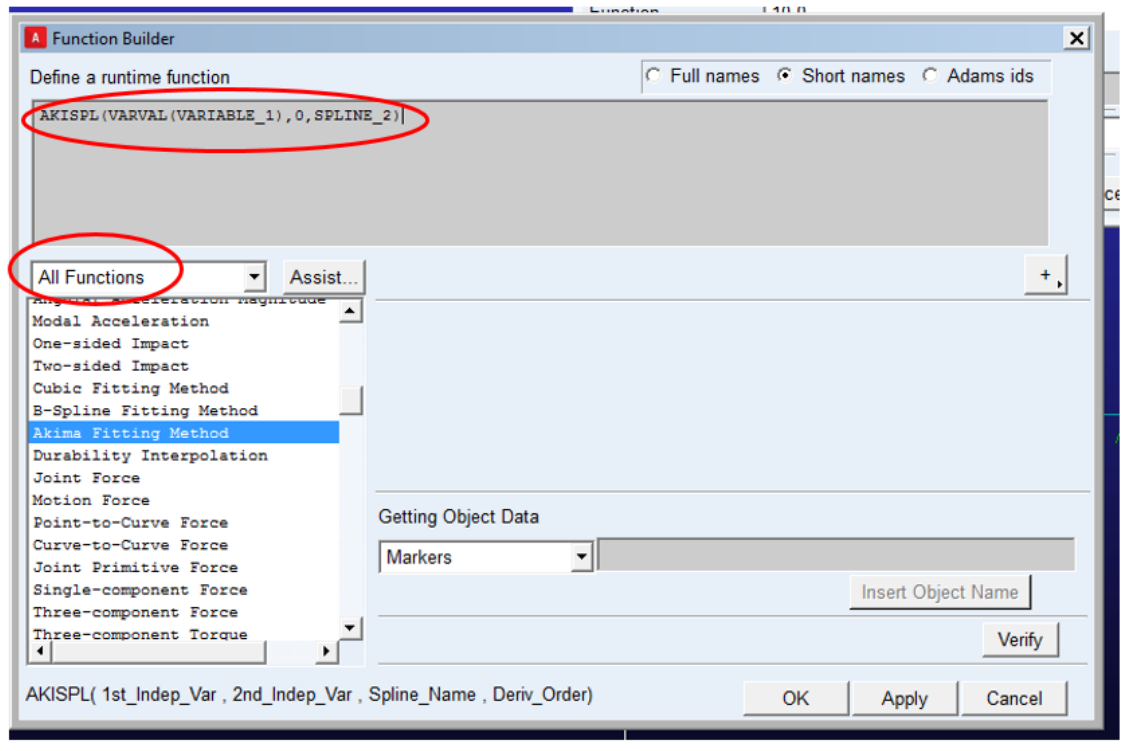

5. You may apply a small resistant constant torque on the output link.

6. Run the simulation. 


\section{G: How to Generate an MNF File}

The units for this example are in SI-units

Part A. ABQUS Analysis

1. Save the CAD cracked gear file as a *.step file

2. Open abaqus 6.13-1, choose "With Standard/Explicit Model"

Create Model Database

With Standard/Explicit Model

3. File $\rightarrow$ Import $\rightarrow$ part $\rightarrow$ browse and find your CAD file

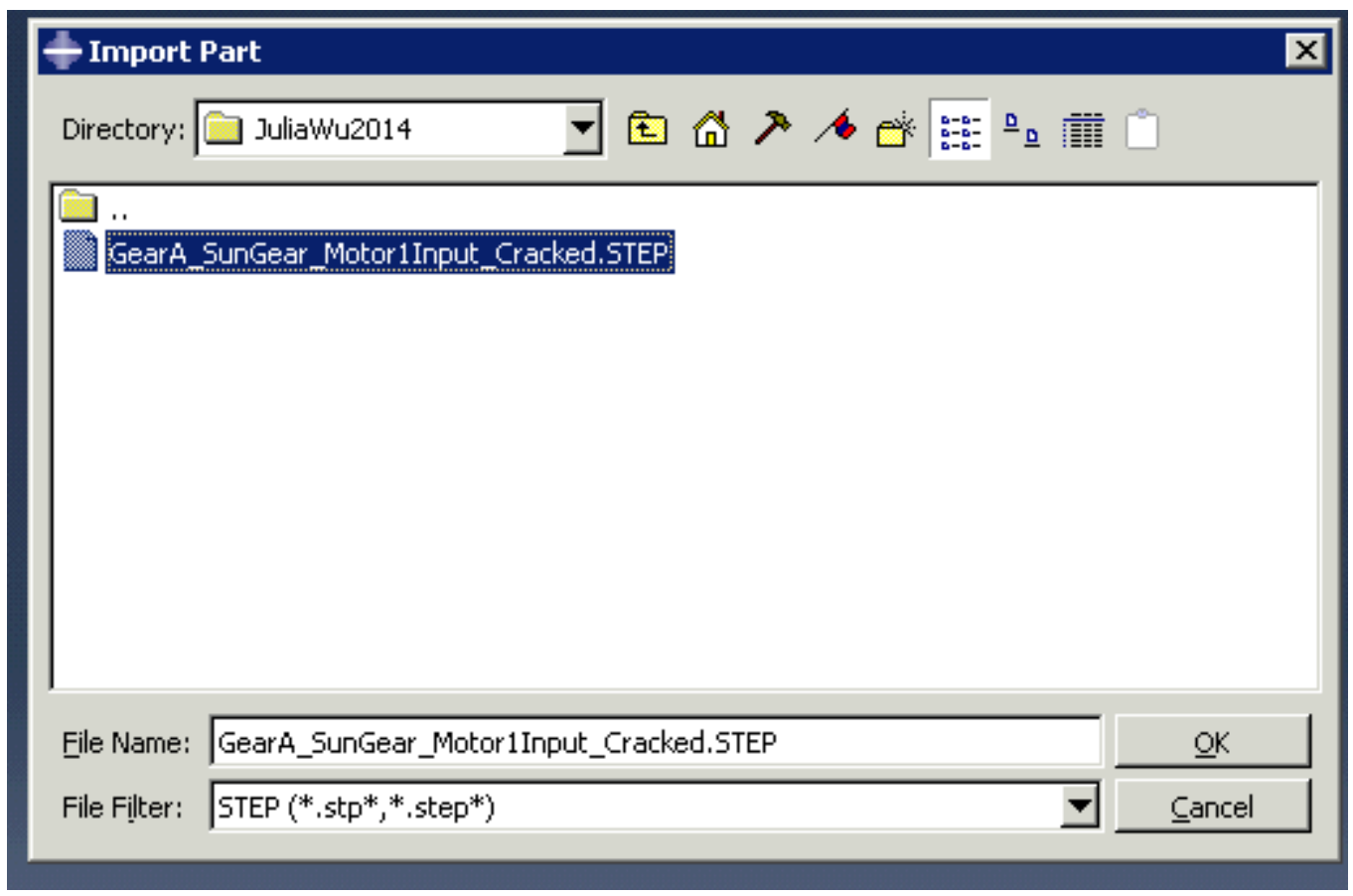

4. Define the material properties. Double click materials in the browse tree.

General -> density type 7800 for steel.

Got to Mechanical ->elastic and type the values shown in: 


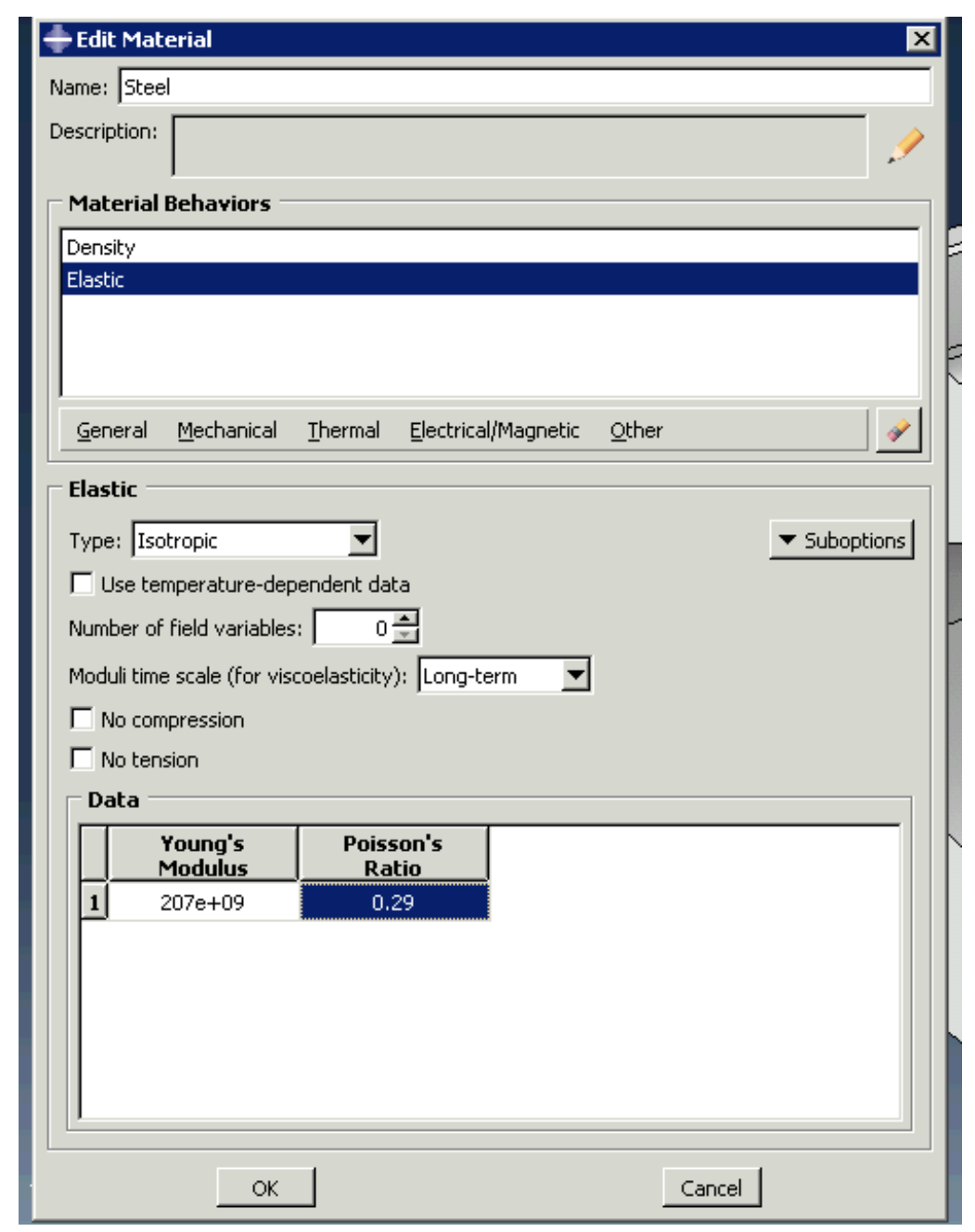

\section{Click OK}

5. Create a section called wu-section, continue, and choose the defined material: steel
宁
Steel
C. Calibrations
宁苛 Sections (1)
$+\frac{\text { Wu-Section }}{+}$

6. Assign a section to the part 密以

Select the entire gear body 


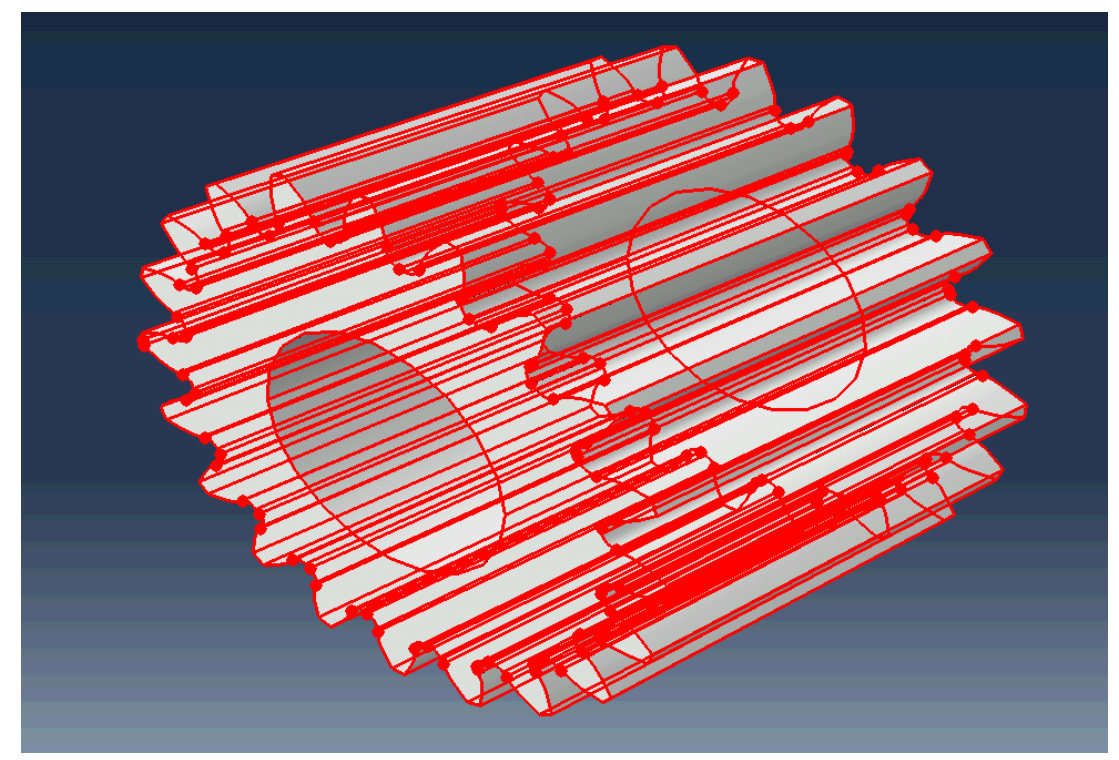

Select the regions to be assigned a section ( $\sqrt{\nabla}$ Create set: $\sqrt{\text { Set-1 }}$ ) Done

Click on done to assign the section.

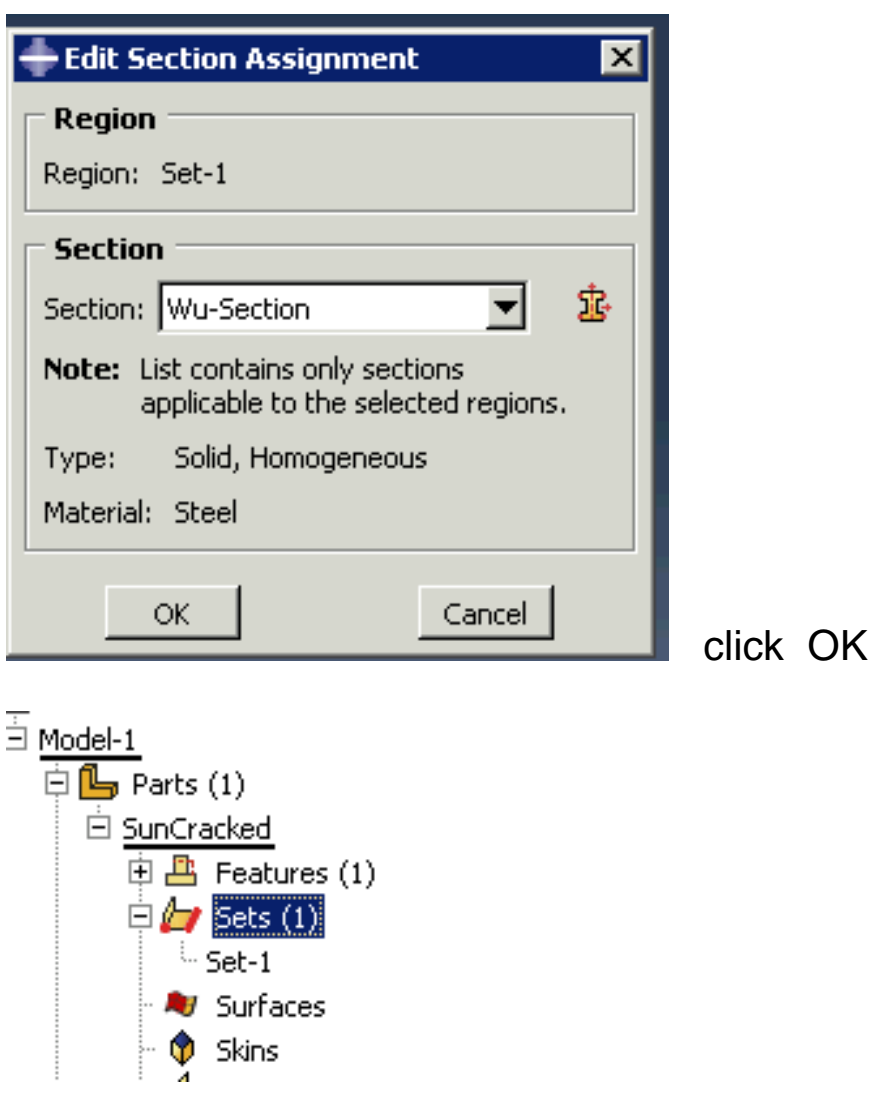


7. Double click on instances under assembly

Choose "independent (mesh on instance)" for complicated geometries.

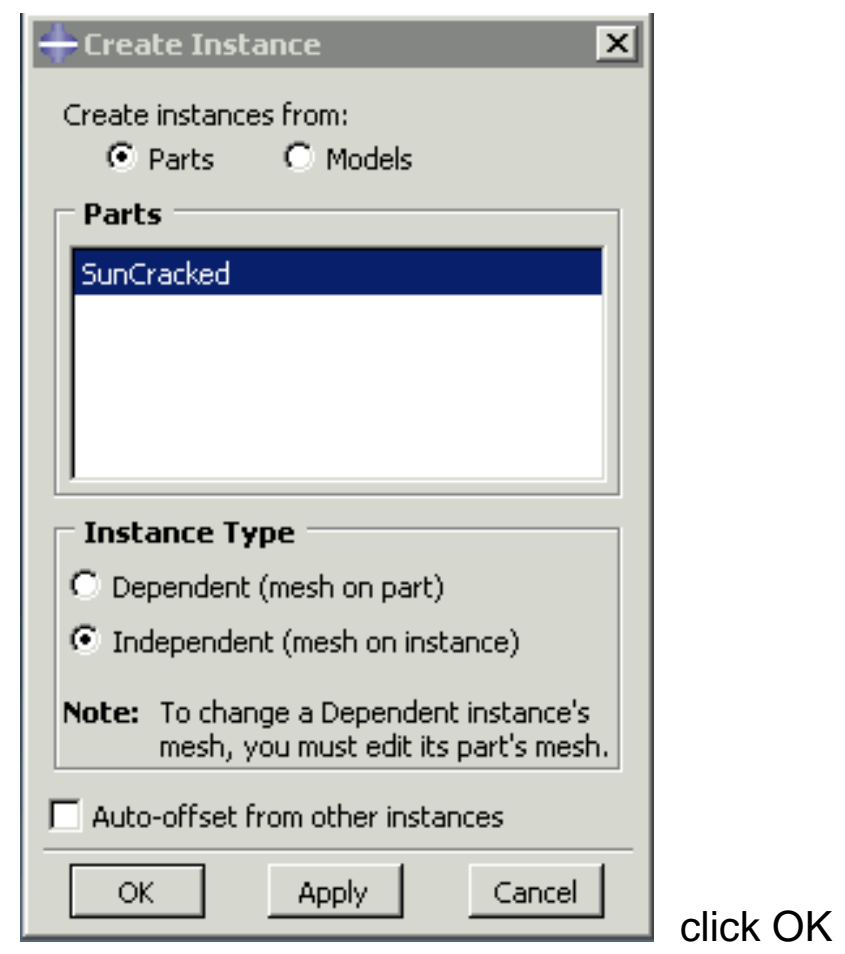

8. double click "mesh under assembly" to mesh on instance, which gives you more freedom to generate the mesh.

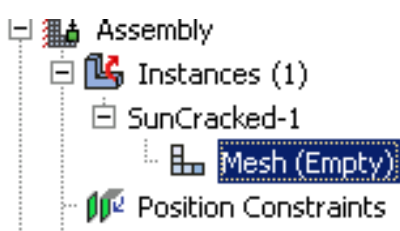

Seed part instance 


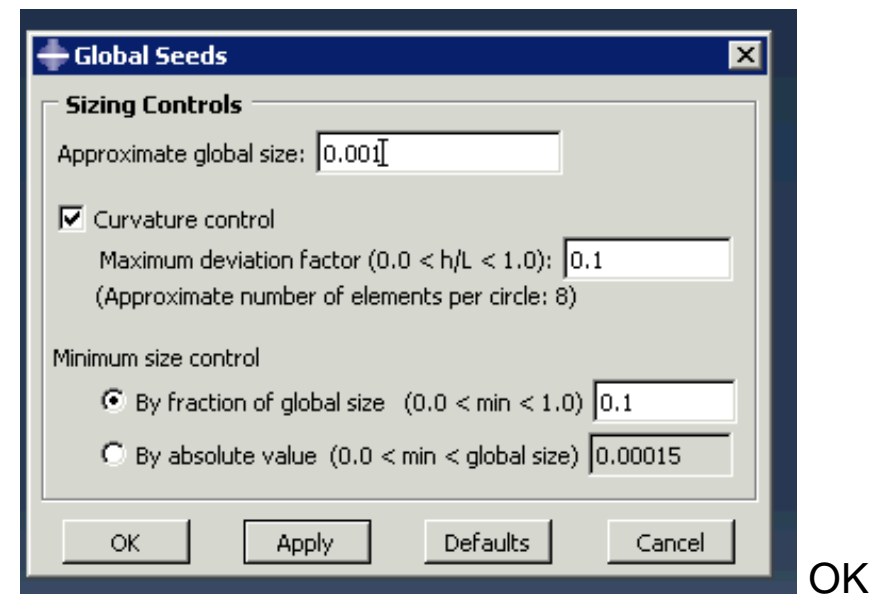

9. Fine seed at the edge of crack.

Click "seed edge" ....

Hold "shift" the key and select the "3 edges" of the crack. Click on Done

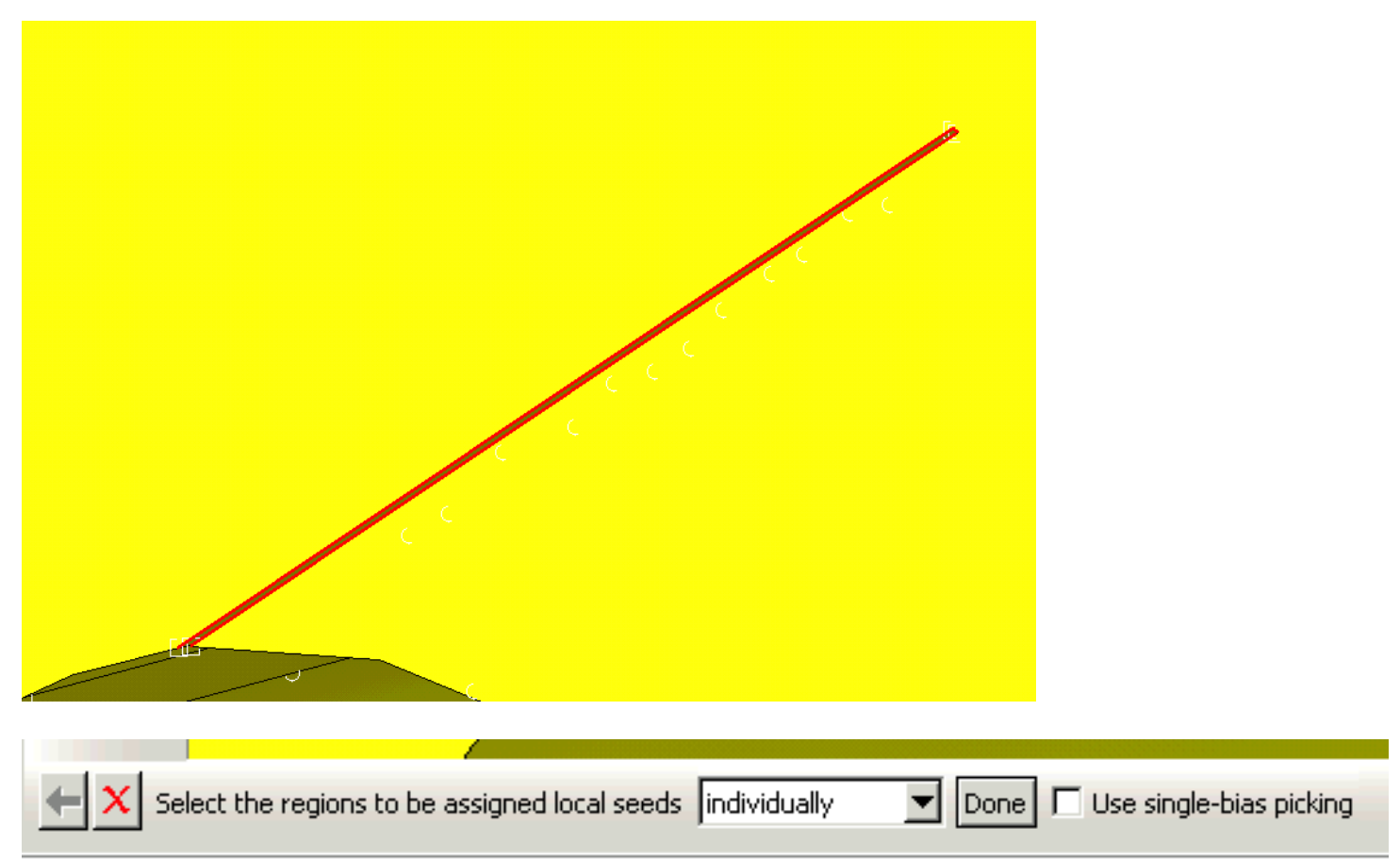




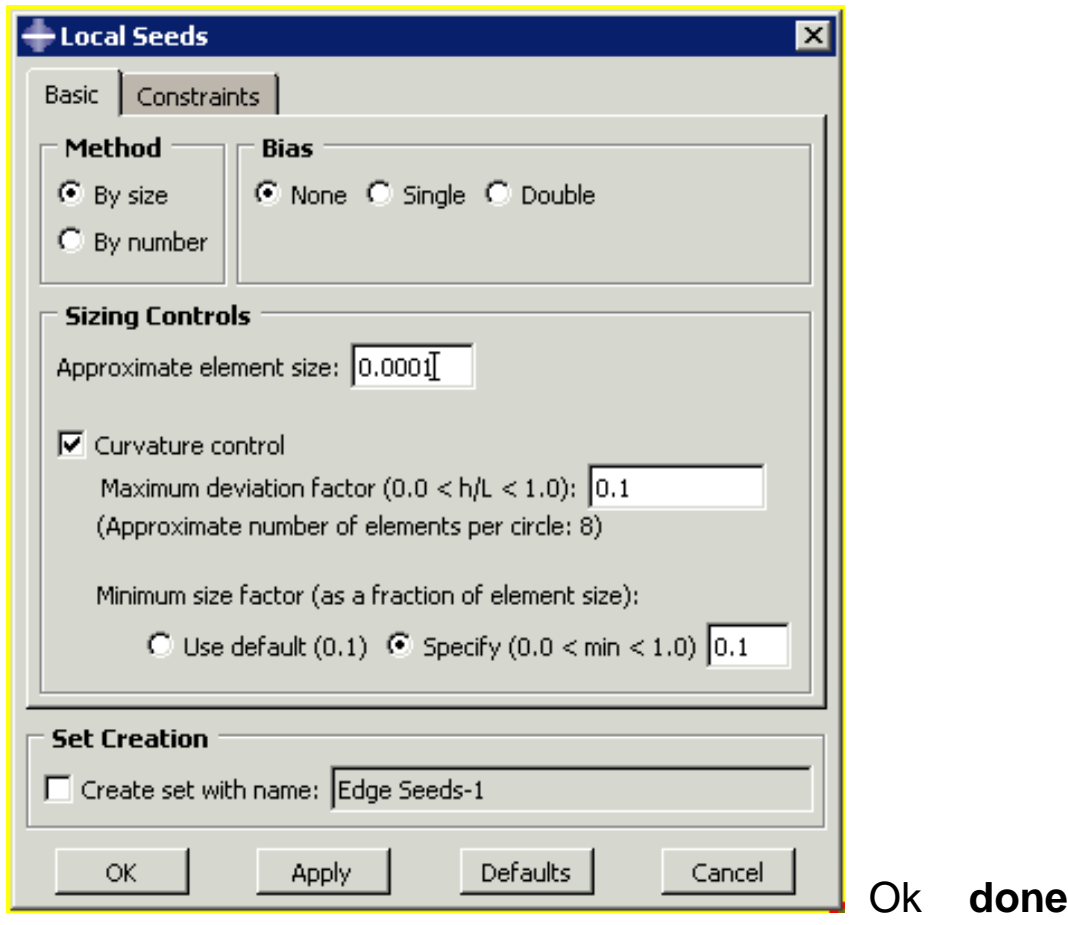

10. “mesh part instance" 睓.

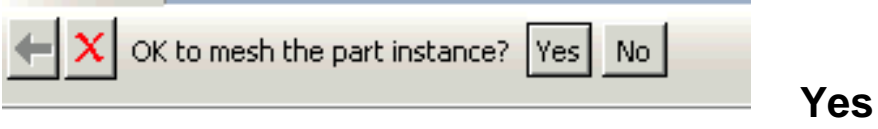

11. If a mesh error shows up:

"seed edges" again. Zoom in the crack, select the shortest edge of the crack. Make the mesh size smaller.

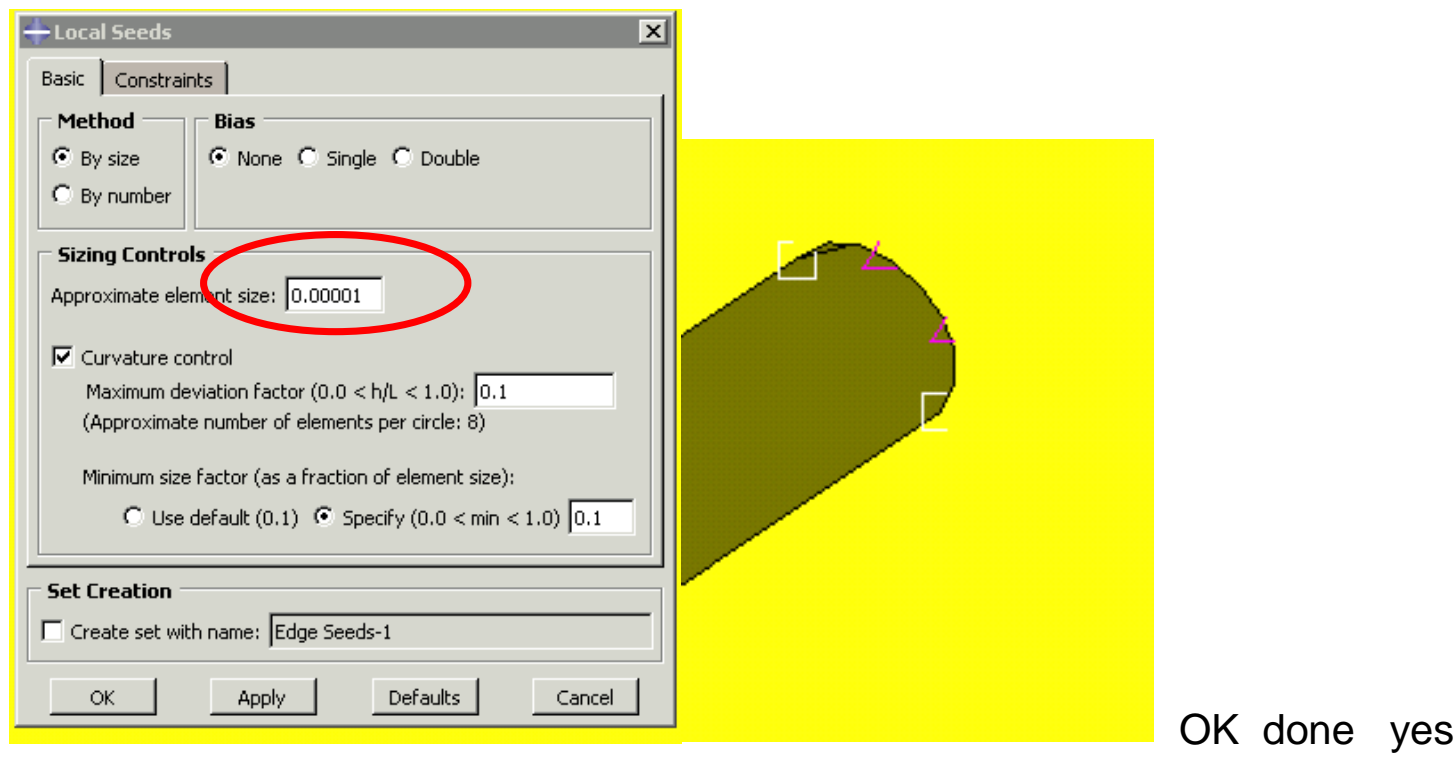


If meshing failed again.

Go to "mesh" on the top menu of Abqus and select "controls"
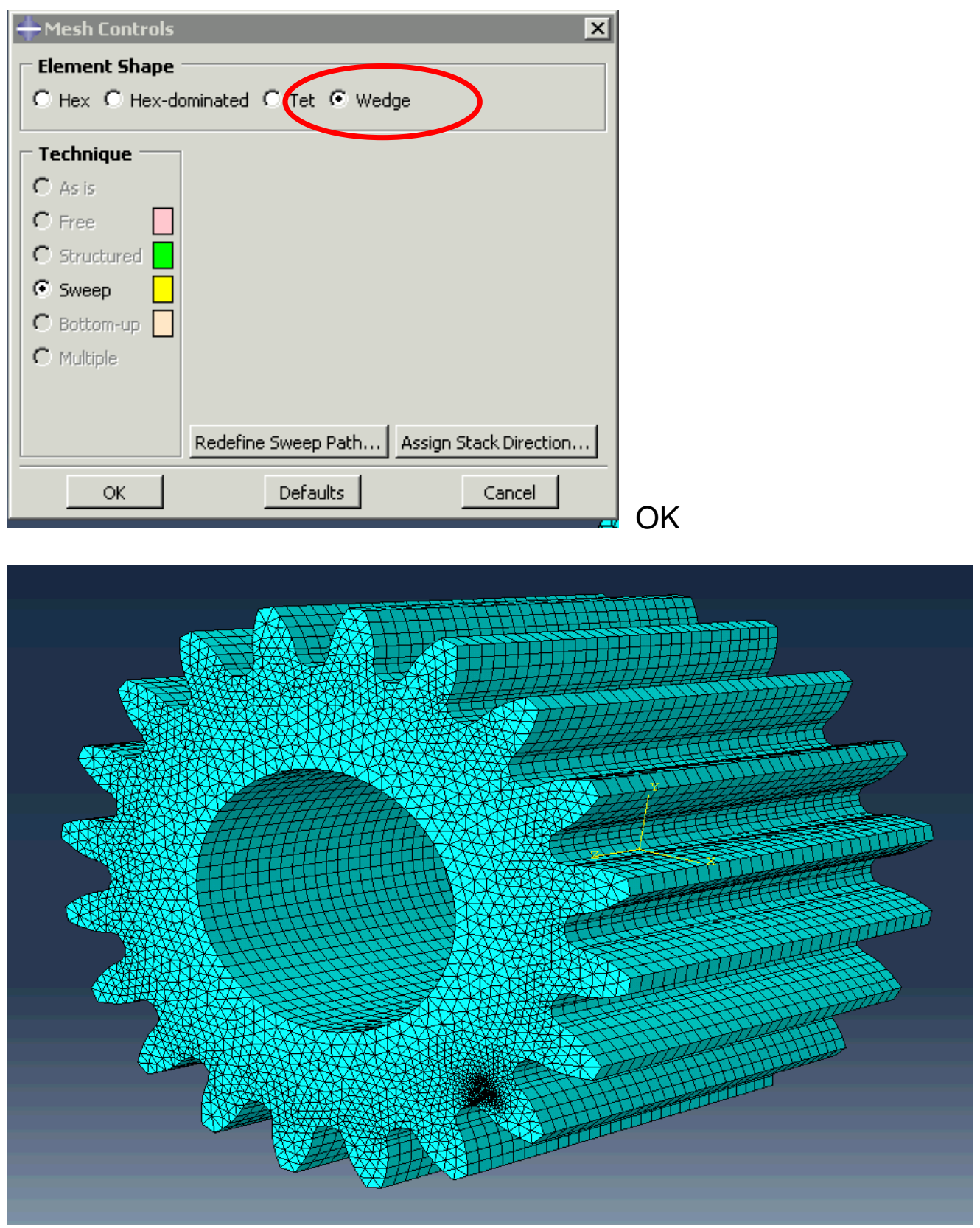

12. Z view

Top menu. Tools $\rightarrow$ set $\rightarrow$ create 


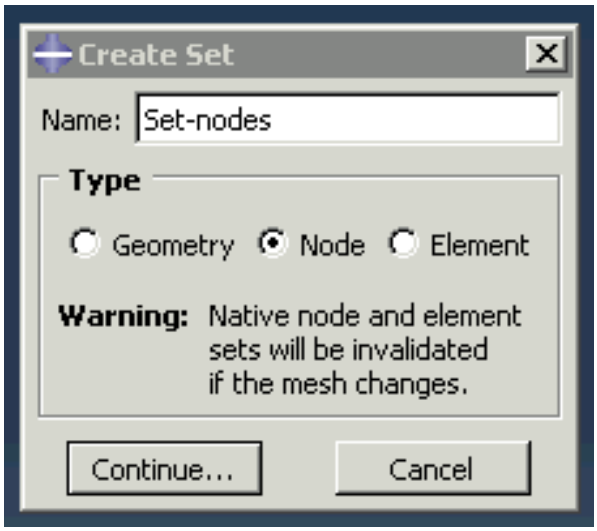

Continue..

On the top menu, left click and hold to expand the menu with a solid triangle on the bottom.

Click $\odot^{+}$and select the inner circle center, then expand the radius to include the nodes of the hole.

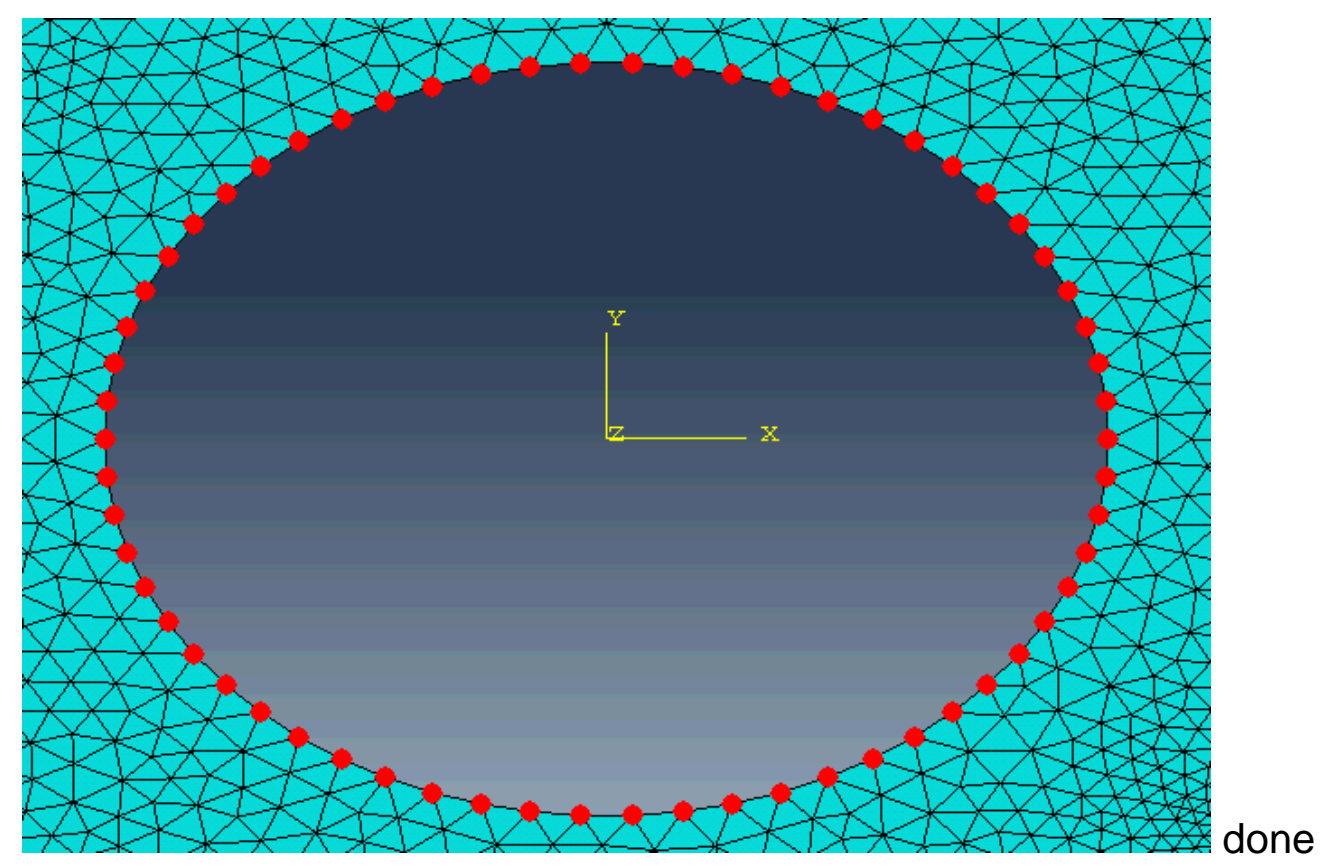

13. create steps

Double click "steps' on the browse tree" 


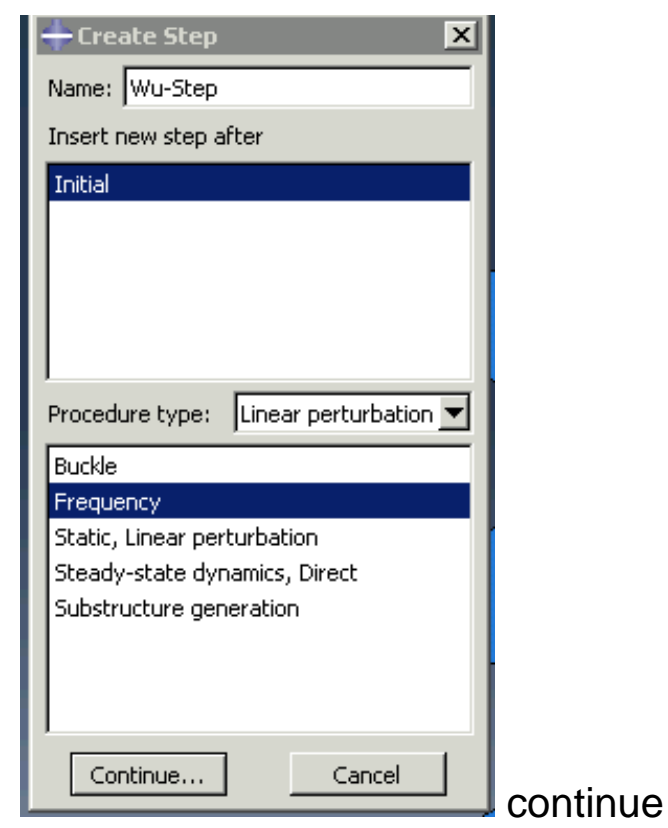

\section{FEdit Step}

Name: Wu-Step

Type: Frequency

Basic Other

Description:

Nigeom: off

Eigensolver: $c$ Lanczos $C$ subspace $C$ aMs

Number of eigenvalues requested: $C$ All in frequency range

$$
\text { c. value: } 20
$$

Г Frequency shift (cyclesitime) ${ }^{* * *} 2:$

ГMinimum frequency of interest (cycles/time):

Г Maximum frequency of interest (cyclesitime):

『 Include acoustic-structural coupling where applicable

Block size: $\odot$ Default $C$ Value:

Maximum number of block Lanczos steps: $\odot$ Defautt $C$ value:

Г Use SIM-based linear dynamics procedures

$\Gamma$ Include residual modes 
14. Make sure the working directory is in your folder by "file $-\rightarrow$ set working directory"

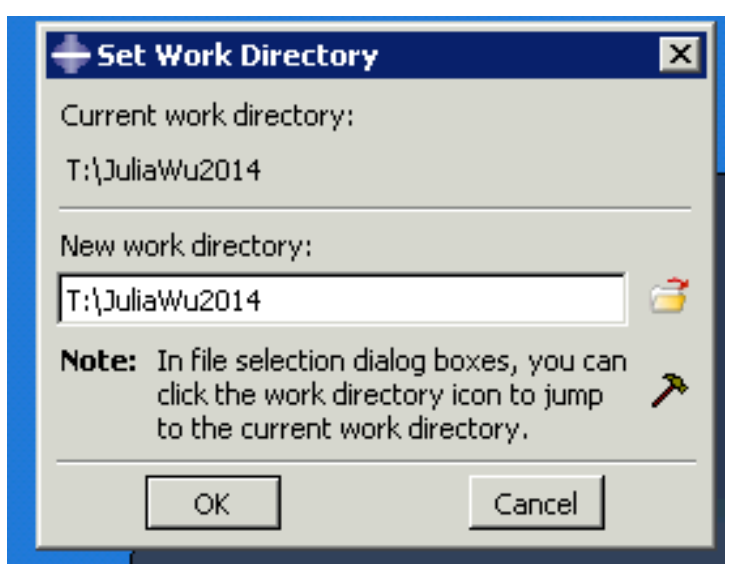

15. Important, copy the file “

ت abaqus_v6.env " in your working directory to increase the space.

16. double click on jobs under analysis

Continue Ok. Right click jobs created $\rightarrow$ submit

17. Save the model and quit ABQUS.

\section{Part 2. Create mnf file from ABAQUS}

18. Copy and paste the following 3 files into the working directory

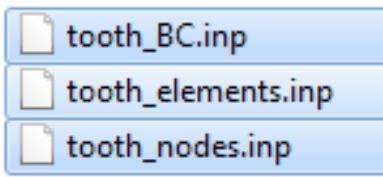

19. Write down the name of the set of nodes you have created in Abaqus. In this case, it is Set-nodes.

20. Open the job file (Job1.inp) as text format.

Open "tooth_nodes.inp" as well. Delete all nodes that are included in this file. Copy the nodes from Job1.inp into "tooth_nodes.inp".

Short cut. Push the bottom "Ctrl + F" to find "element". This is the easiest way to find the end of the nodes. 
(1). Click on the end of the node and hold "Shif" key. Go up to the beginning of the nodes and click. In this way, you select all the nodes.

(2). "Ctrl+X" to delete and copy all the nodes at the same time.

(3). Repeat the above procedure in "tooth_nodes.inp" to select the old nodes. Then past the new nodes there.

(4). Important. Check and make sure no space at the bottom and top is present. Also, no offset of the lines should appear.
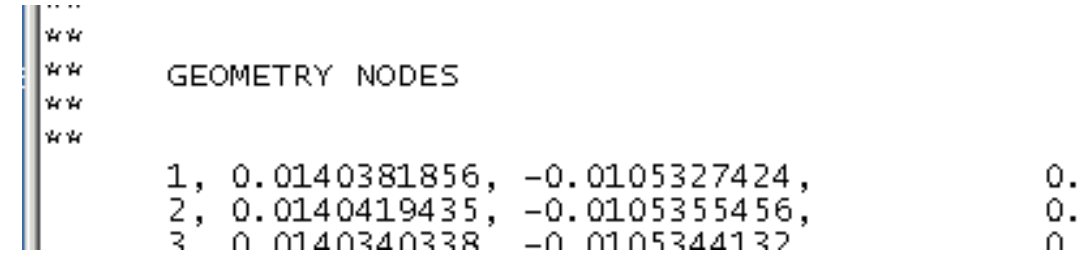

21. "MPC NODES" means Multi-points constraint.

Notice there are two large numbers of the nodes in order to avoid a conflict with one of the current nodes. Delete one if necessary, because only one set of nodes is defined ABAQUS.
10000000
20000000
o.,
0.
0 .
$0 ., 1$.

Change $z$ coordinate as $0.015 \mathrm{~m}$ which is the half of the width of the fear.
$\mid \begin{aligned} & k w \\ & k w \\ & k w \\ & k w\end{aligned}$
MPC NODES
10000000 ,
o.,
0. ,
0.015

Make sure there is no space. Save and close.

22. Repeat the same procedure for elements.

Open "tooth_elements.inp" delete the old elements. Copy and paste the elements from "Job1.inp" into this file. Save and close. Press "Ctrl+F" Setnodes". Make sure no back space is appearing at the top or the bottom.

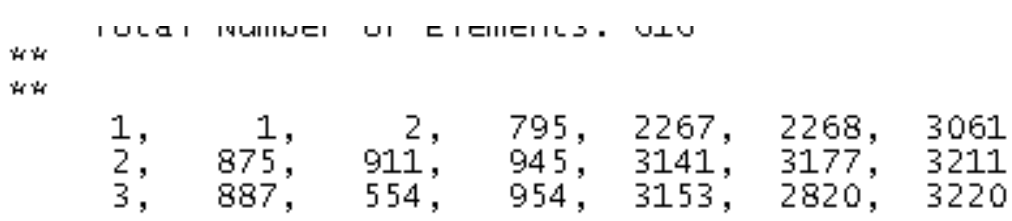

23. Open "tooth_BC.inp"

(1). Change the file name 
"HEADING

SUN

Notice, "SUN" should be the ABAQUS file name for your model.

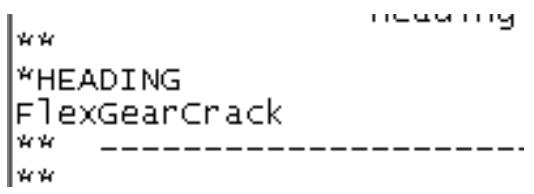

(2). Define the constraint node sets

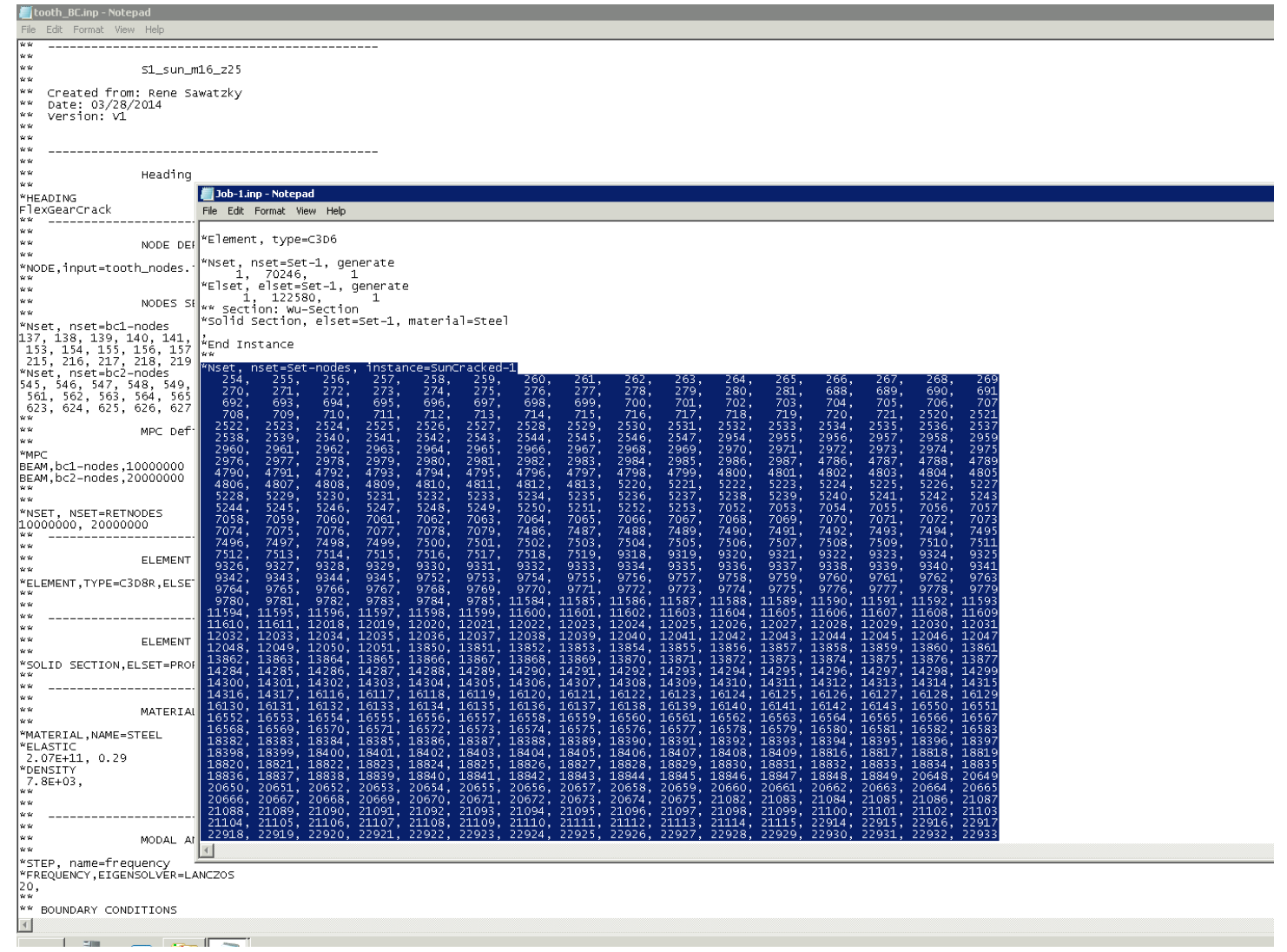

Select nodes set including "*Nset ..." and paste it into this file.

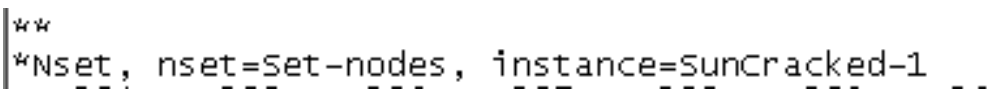

If "instance $=. .$. " shows up, delete it and the coma, before it in order to avoid any errors while excuting later.

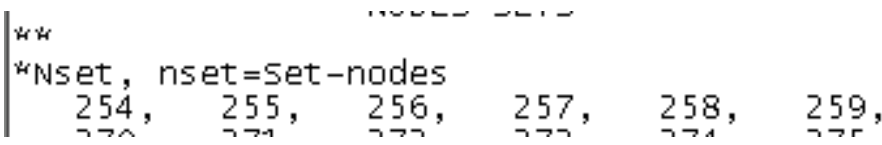


(3). Define the beams to connect constraint nodes to MPC node

Old format with two constraint sets and MPCs

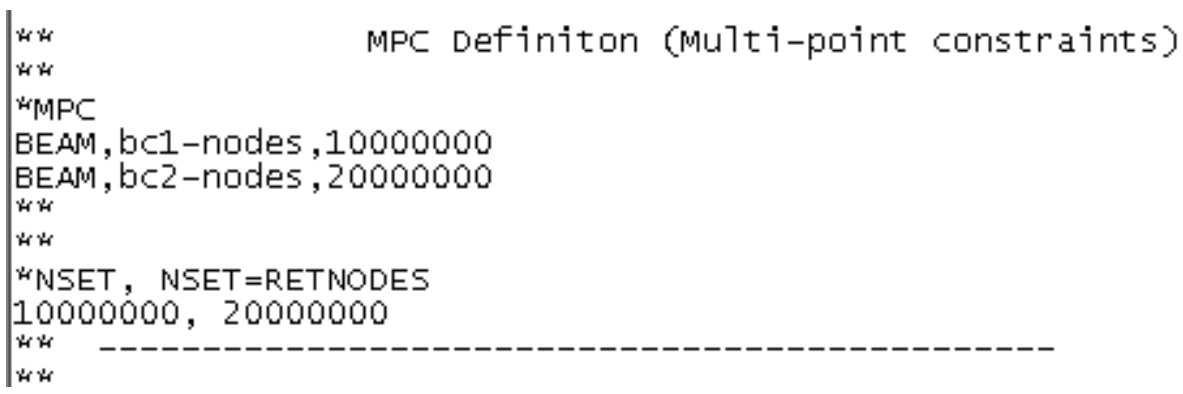

Your case has one constraint set therfore one MPC node.

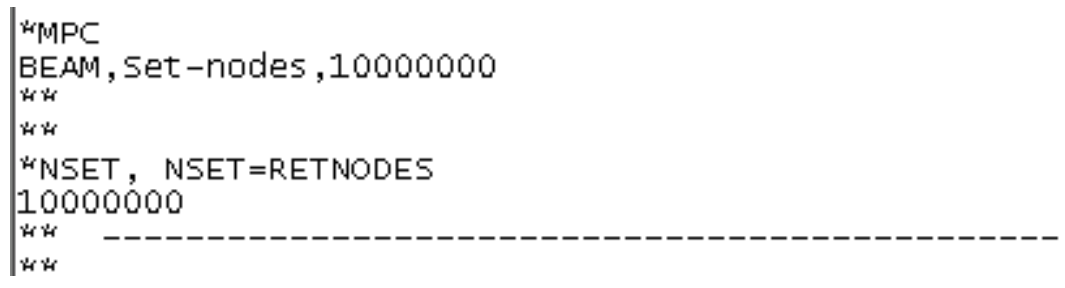

(4). Check and modify the element type

Old format "TYPE=C3D8R"
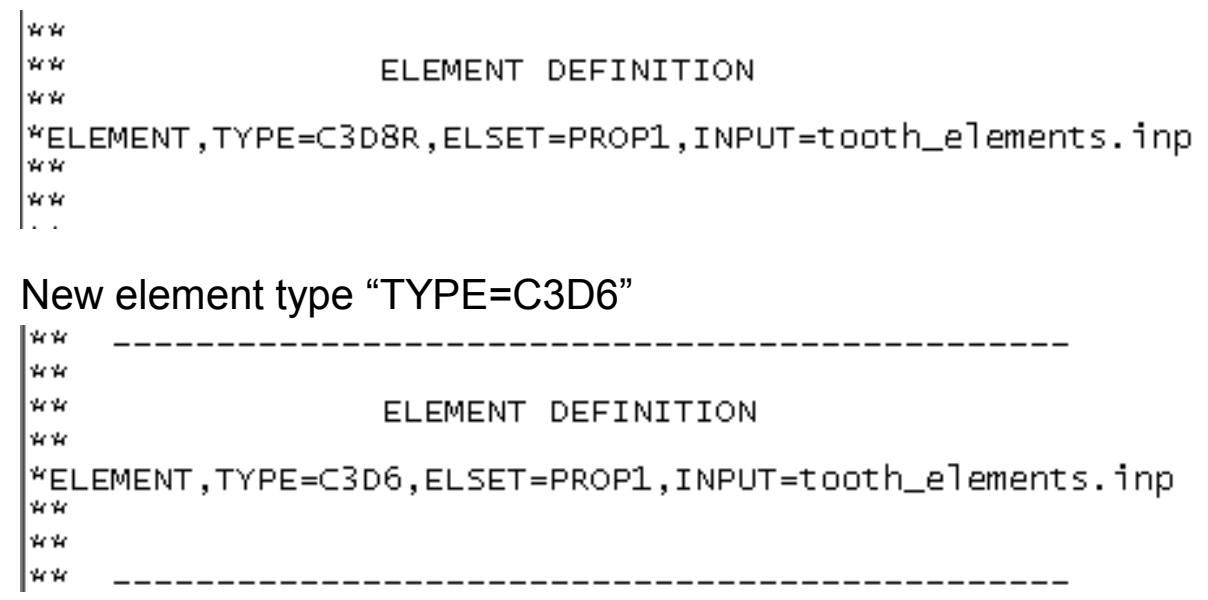

(5) Check and modify the material properties, if necessary.

In this case, steel appears, which is the same as defined in Abaqus.

(6). Save "tooth_BC.inp", but DO NOT save "Job1.inp" 
24. Open Abaqus Command

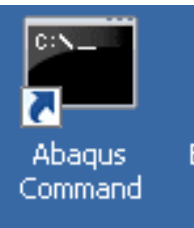

Command window.

Copy the working directory into the

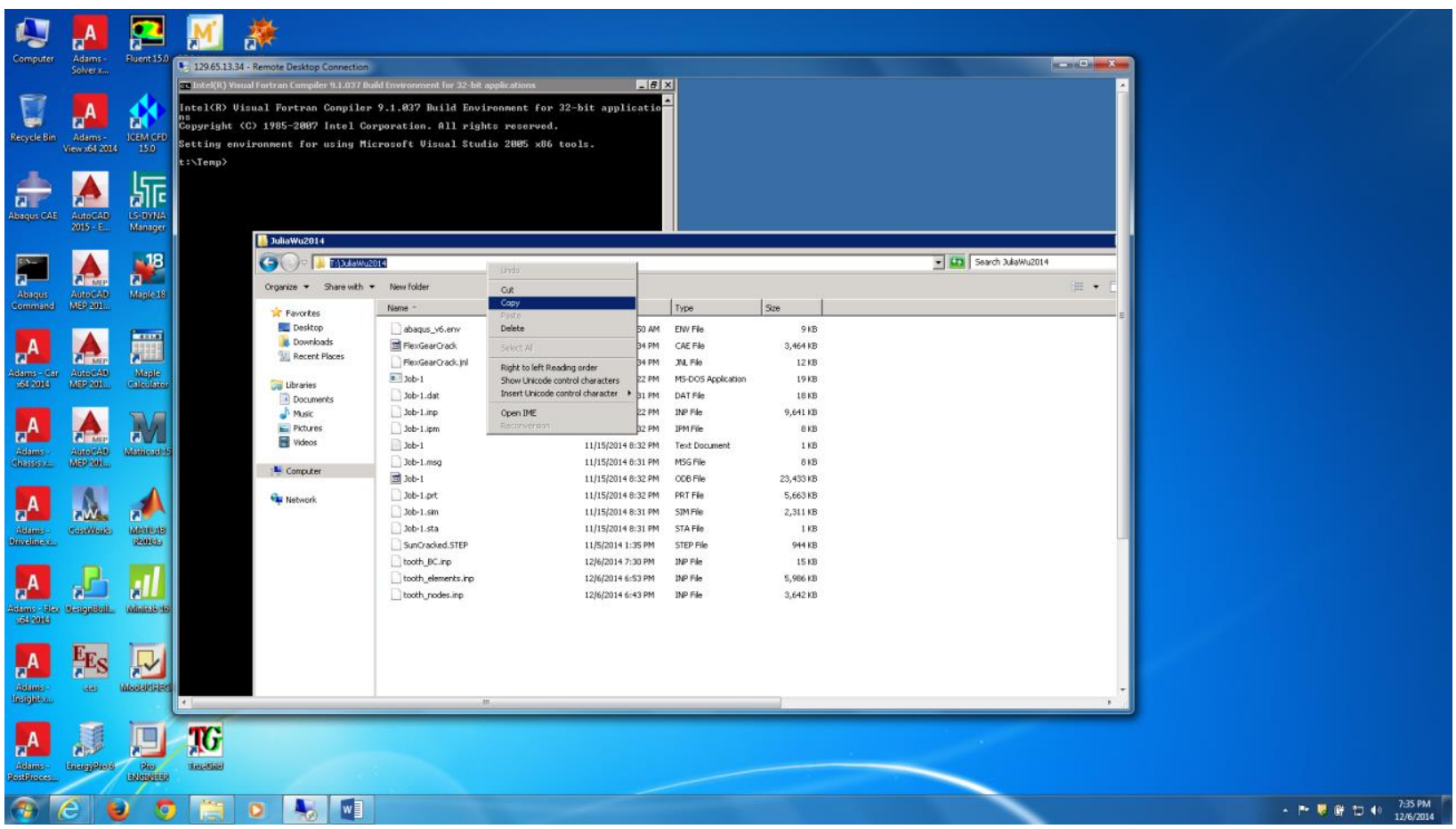

(1) Define the working directory with cd lworking directory

$$
\begin{aligned}
& t: \backslash \text { Temp }>c d ~ T: \backslash J u l i a W u 2014 \\
& \text { T : \JuliaWu2014〉 }
\end{aligned}
$$

(2) Enter following code:

abaqus job=tooth_BC interactive

: $\backslash$ JuliaWu2014 $>$ abaqus job=tooth_BC interactive

wait until message appears: COMPLETE

(3) Enter following code:

(abaqus adams job=filename_BC substructure_sim=filename_BC_Z1 model_odb units=Mks) 
(4) Message will come asking you enter for deault or none

(5) Enter for yes

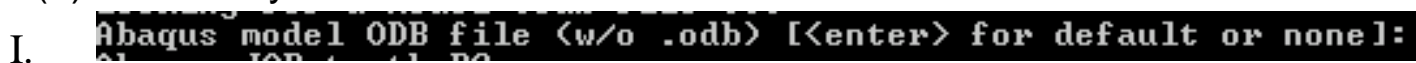

(6)

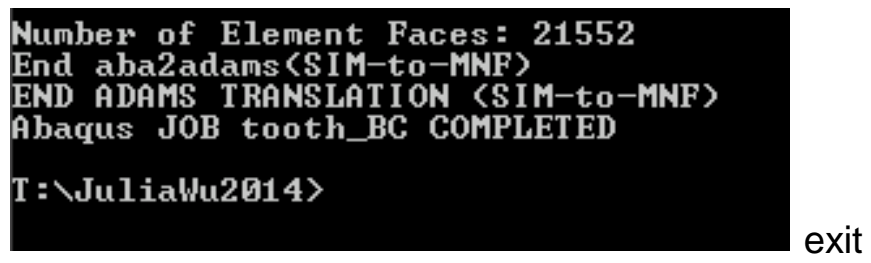

25. Go to the working directory and copy the .mnf file and past it into your own file.

tooth_BC.mnf $12 / 6 / 20148: 09 \mathrm{PM}$

MNF File

$89,035 \mathrm{~KB}$

Part 3. Import mnf file into ADAMS to create the model

26. Open ADAMS view, import mnf file. Change the units as SI which must be consistent with ABAQUS.

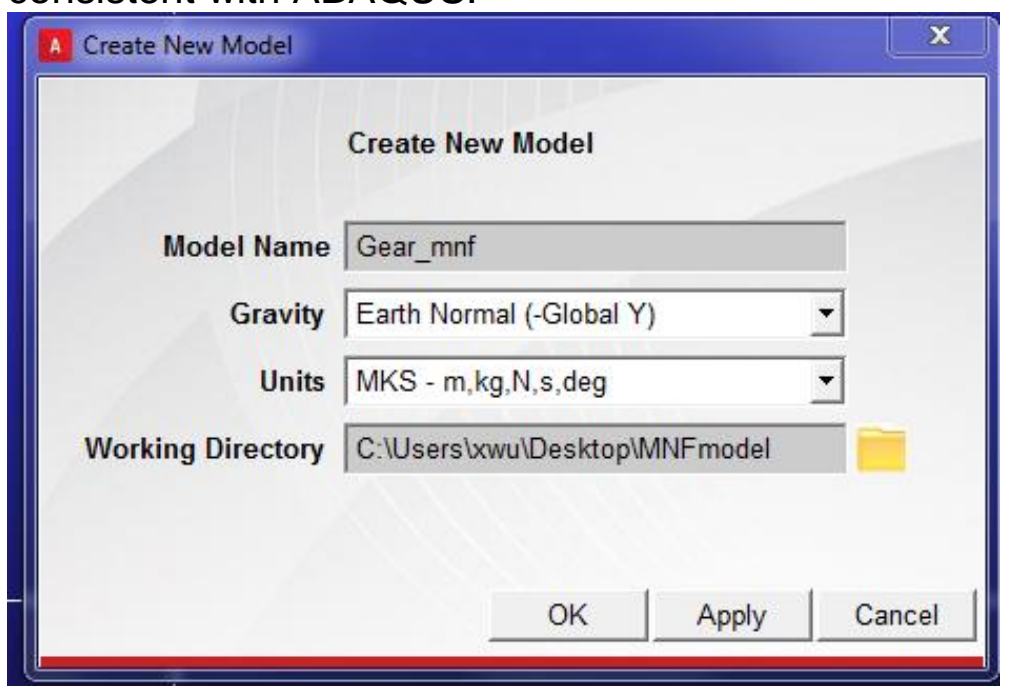

27. This step must be done before importing the model. You have to enter this command in the Command Window to reduce the tolerance scale: defaults geometry display_tolerance_scale $=0.00001$ for $\mathrm{SI}(\mathrm{m})$ units.

28. Import *.mnf file first. Select

ADAMS/flex, create flex body through mnf import under body. 


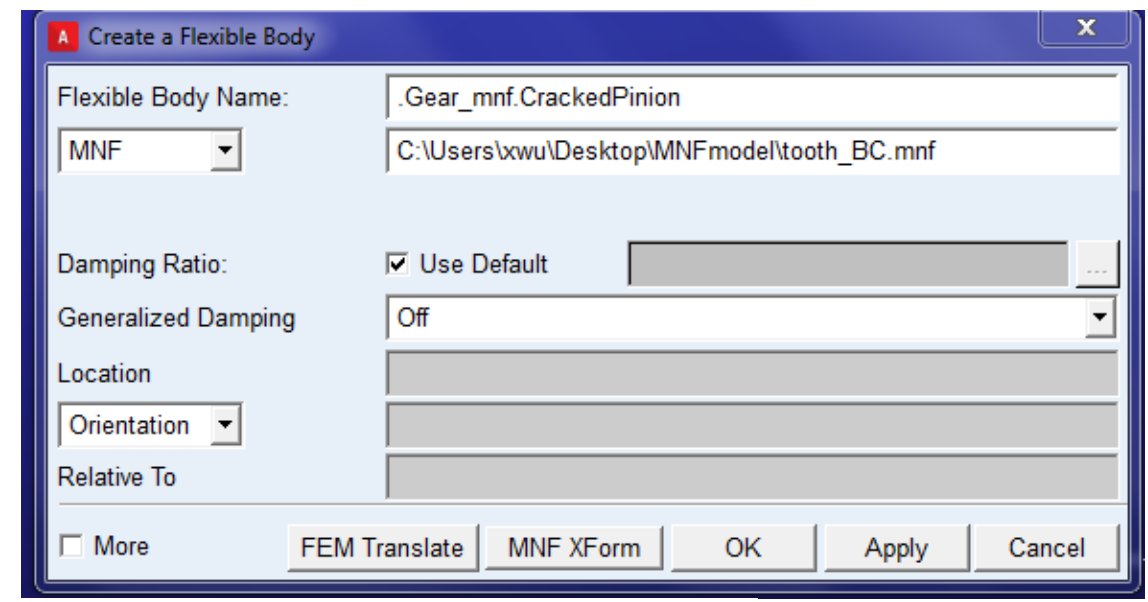

\begin{tabular}{ccc}
\hline Planetary & 37 & 74 \\
Sun & 20 & 40
\end{tabular}

The distance between sun and planet is $57.2 \mathrm{~mm}$

28. Import the planet as Gear (part name)

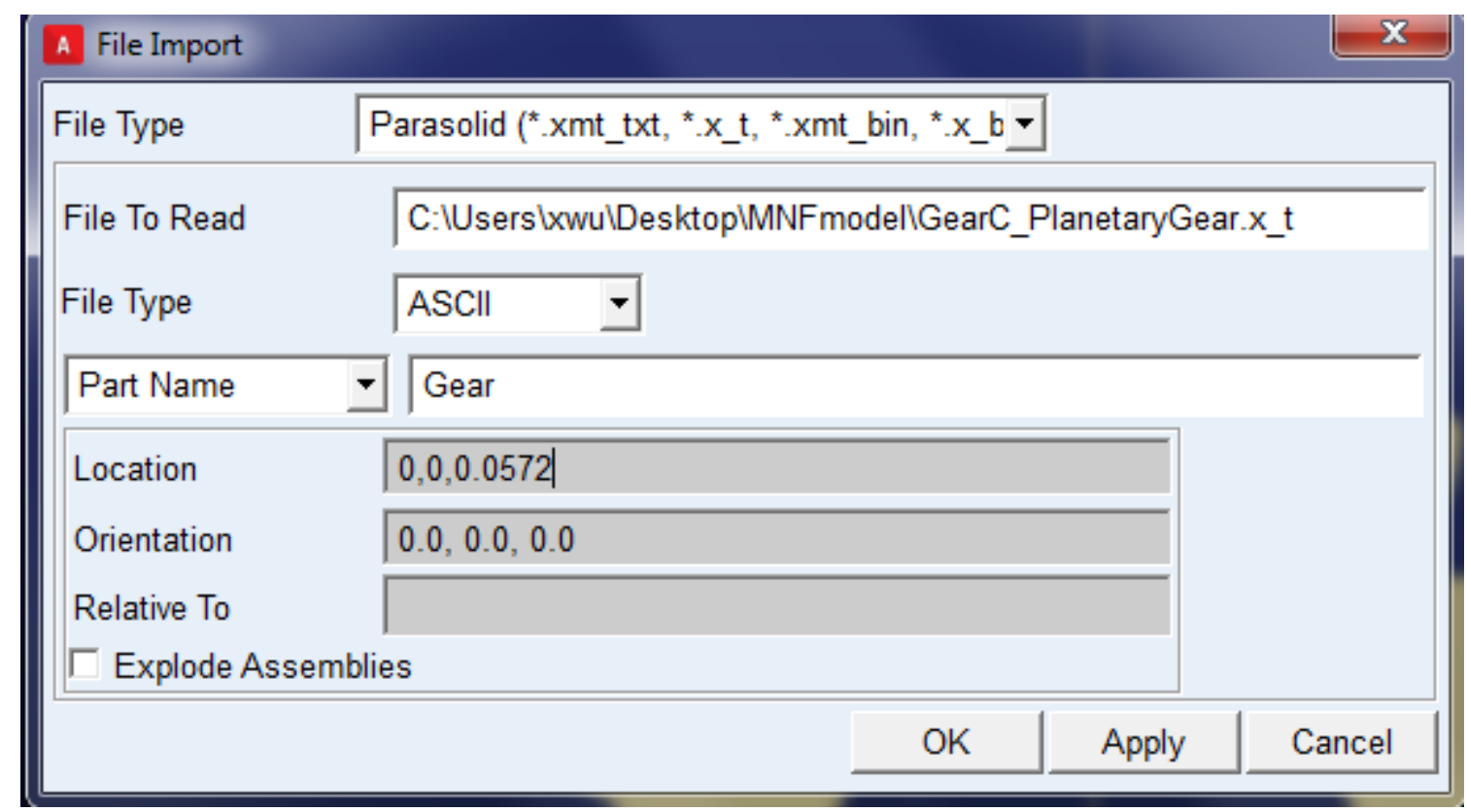

29. Define the material properties for the Gear. 


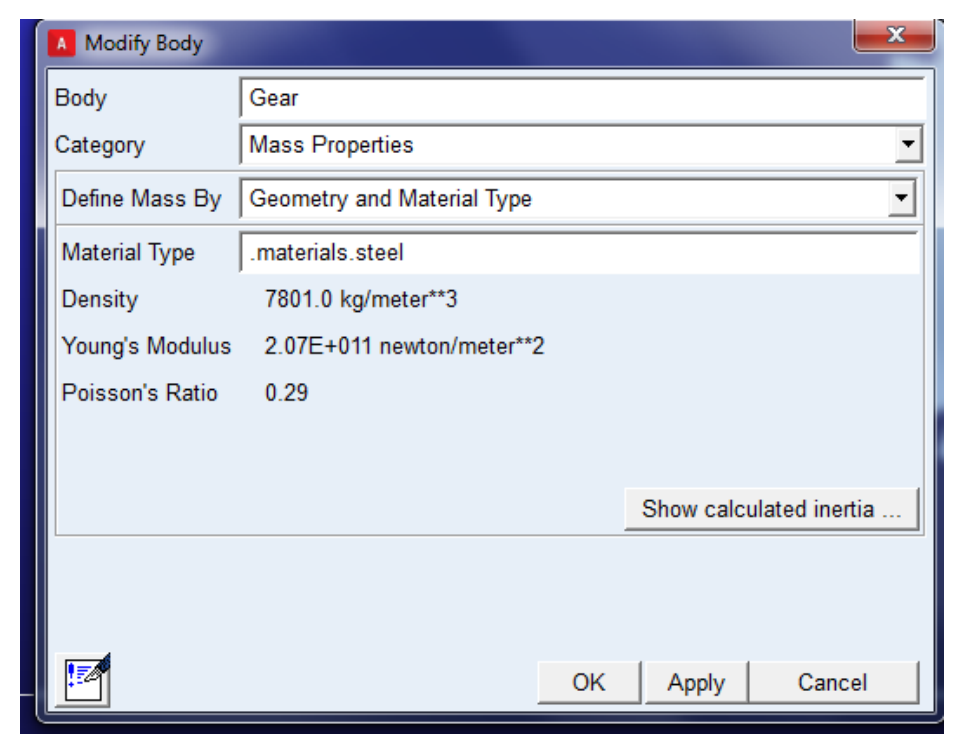

30. Define the revolute joints and apply the motion on the sun.

31. Define the contact between gears. Choose FlexBody to Solid as Contact Type

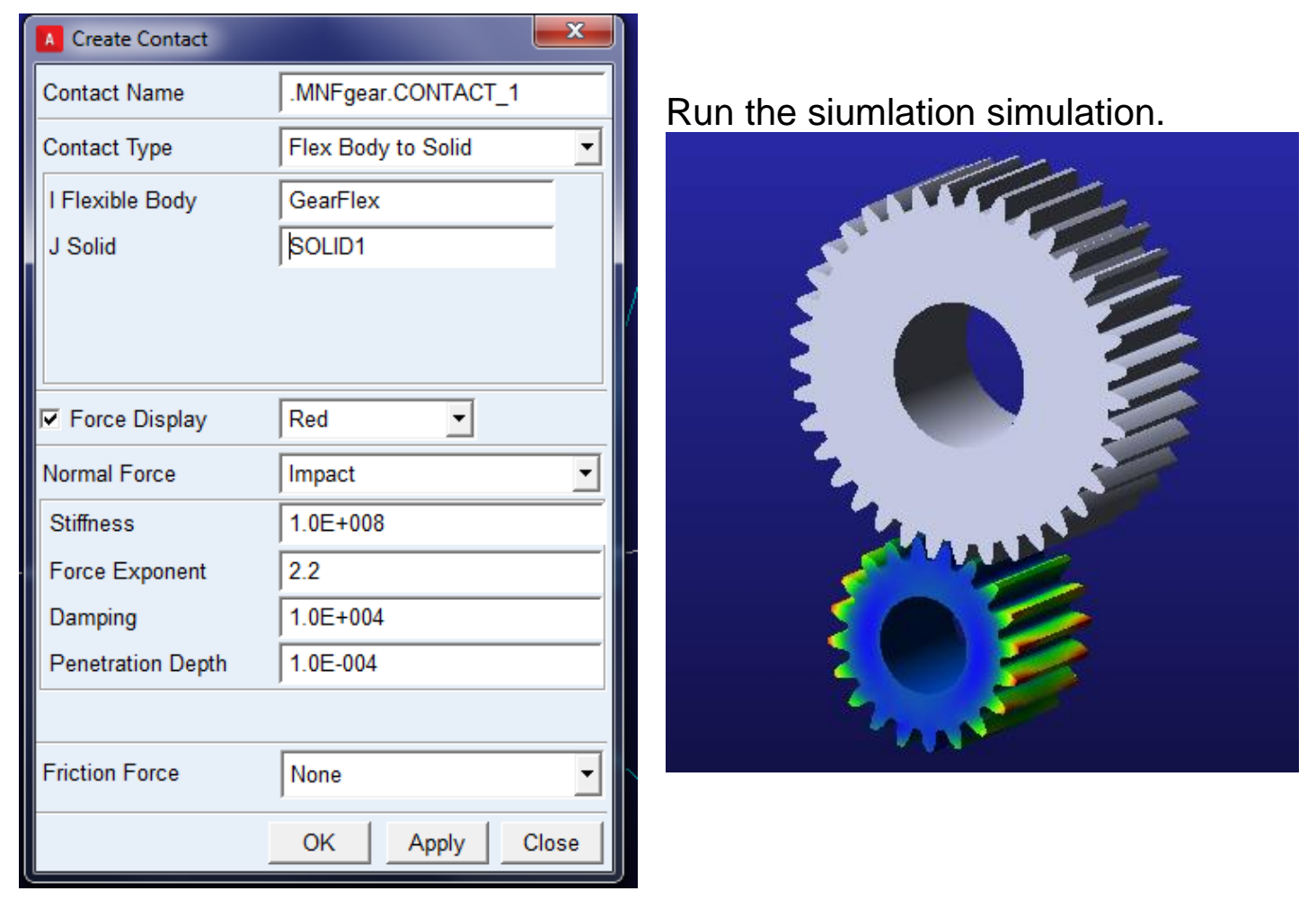

